\title{
DATA MODEL AND RELATIONAL DATABASE DESIGN FOR THE NEW JERSEY WATER-TRANSFER DATA SYSTEM (NJWaTr)
}

Open-File Report 03-197

Prepared in cooperation with the NEW JERSEY DEPARTMENT OF ENVIRONMENTAL PROTECTION

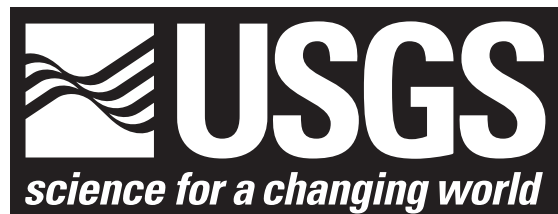





\section{DATA MODEL AND RELATIONAL DATABASE DESIGN FOR THE NEW JERSEY WATER-TRANSFER DATA SYSTEM (NJWaTr)}

By Steven Tessler

U.S. GEOLOGICAL SURVEY

Open-File Report 03-197

Prepared in cooperation with the NEW JERSEY DEPARTMENT OF ENVIRONMENTAL PROTECTION

West Trenton, New Jersey 2003 


\section{U.S. DEPARTMENT OF THE INTERIOR \\ GALE A. NORTON, Secretary}

\section{U.S. GEOLOGICAL SURVEY \\ Charles G. Groat, Director}

The use of trade names in this report is for identification purposes only and does not constitute endorsement by the U.S. Government.

For additional information write to:

District Chief

U.S, Geological Survey

Mountain View Office Park

810 Bear Tavern Road, Suite 206

West Trenton, NJ 08628
Copies of this report can be purchased from:

U.S. Geological Survey Branch of Information Services Box 25286

Denver, CO 80225-0286 


\section{CONTENTS}

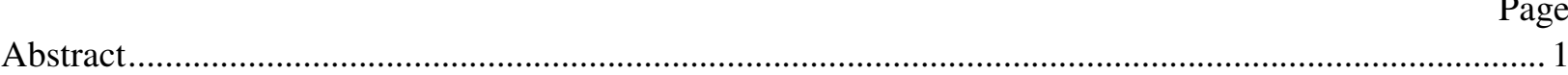

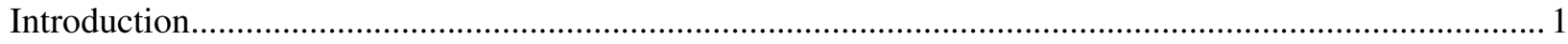

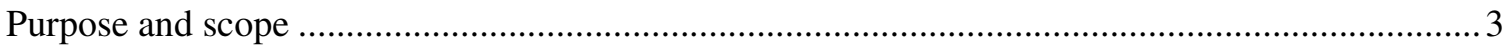

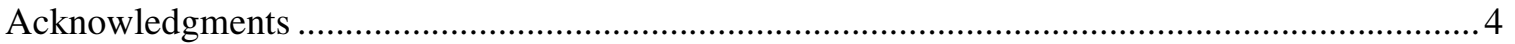

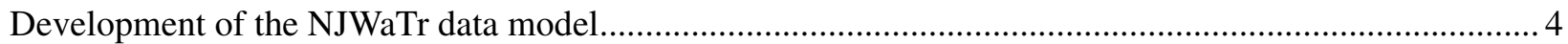

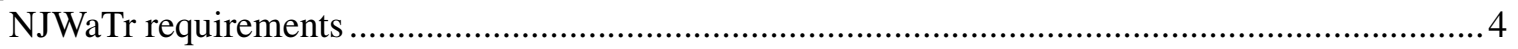

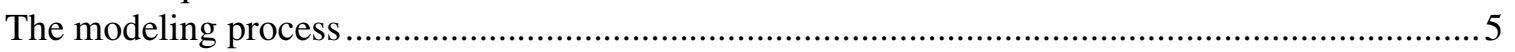

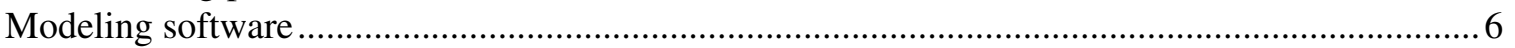

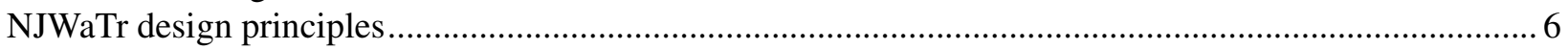

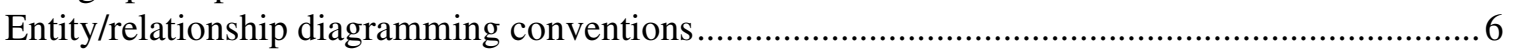

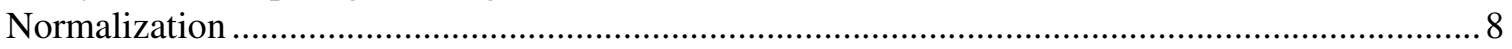

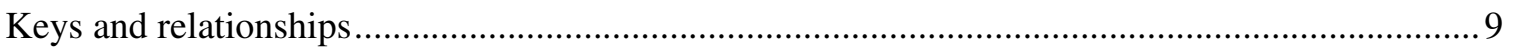

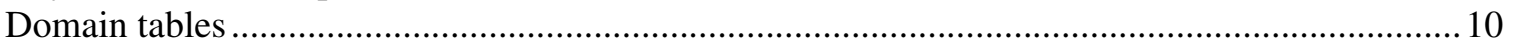

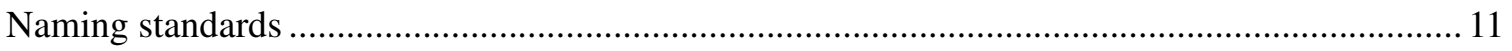

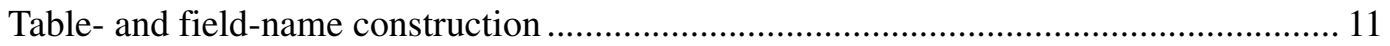

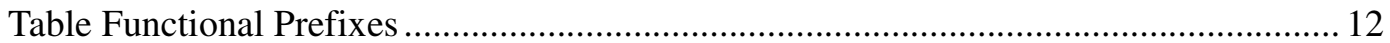

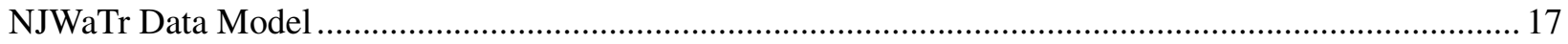

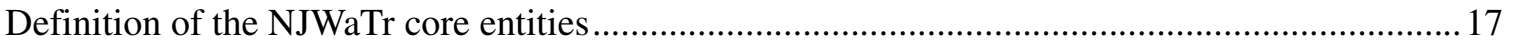

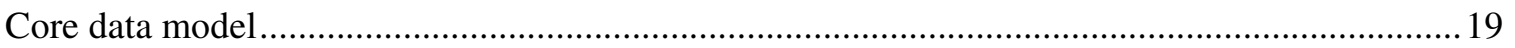

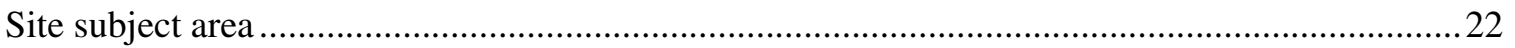

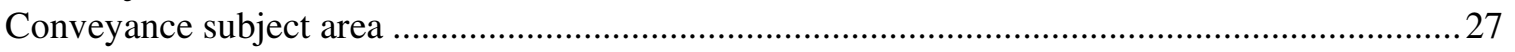

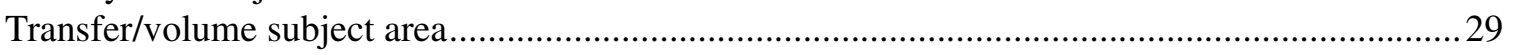

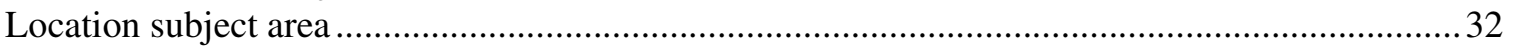

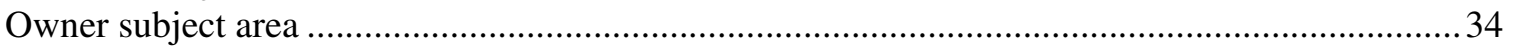

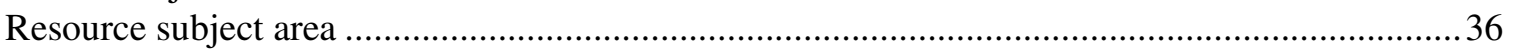

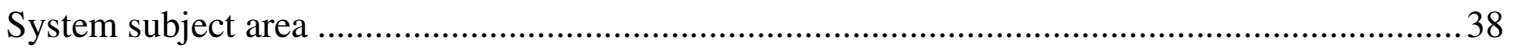

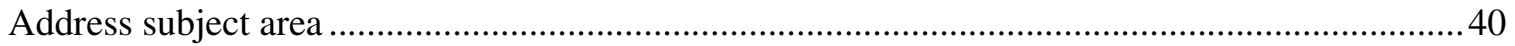

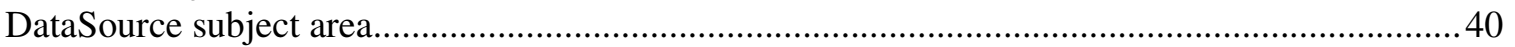

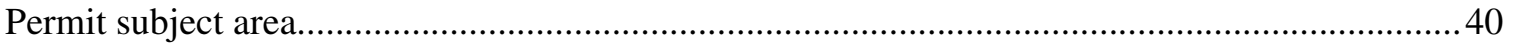

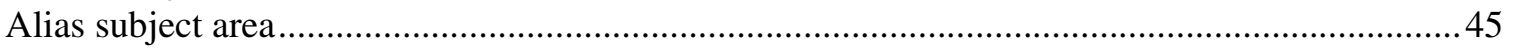

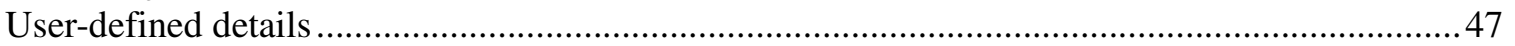

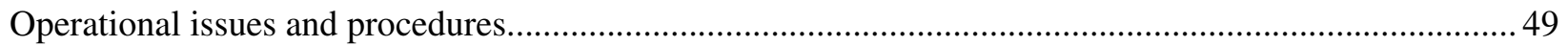

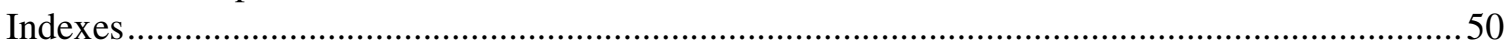

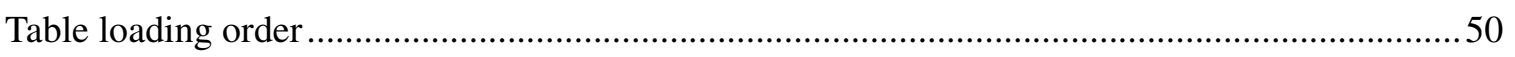

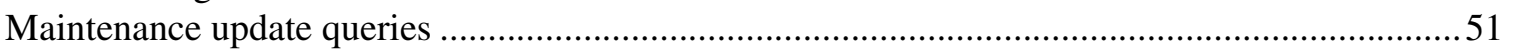

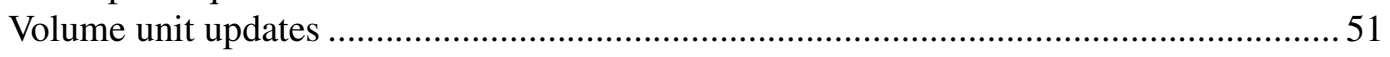

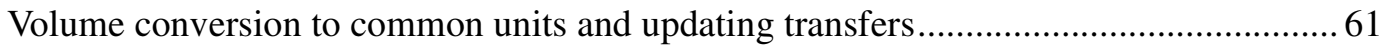

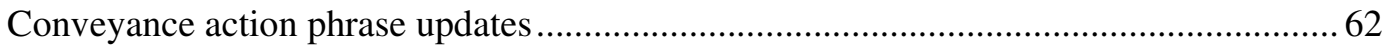

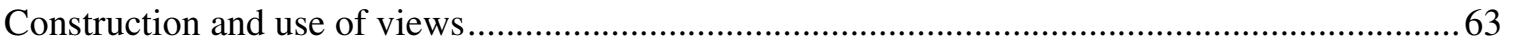

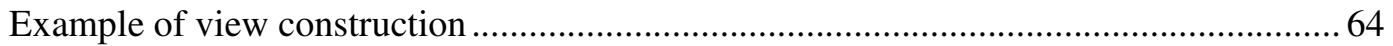

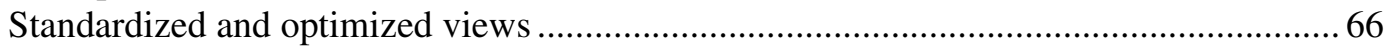

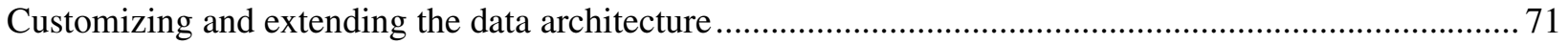

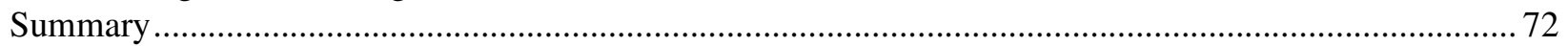

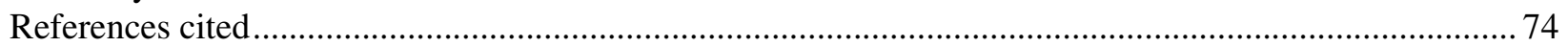

Appendix 1. Data dictionary for the New Jersey Water-Transfer Data System (NJWaTr) ......................1-1

Appendix 2. NJWaTr domain table listings by subject area ................................................................ $2-1$

Appendix 3. Comparison of the NJWaTr and NEWUDS data models ............................................... 3-1 


\section{PLATE}

Data model and relational database design for the New Jersey water-transfer data system (NJWaTr)

\section{ILLUSTRATIONS}

Figures 1-5. Diagrams showing:

1. Graphical representation of entities, attributes, and relationships (E/R diagram) ....... 7

2. Examples of tables with functional prefixes and their diagram display formats........ 13

3. Representation of a small water network consisting of a withdrawal well, a treatment plant, and two distribution systems, along with their unidirectional conveyances and four estimated daily transfer/volume values for one conveyance

4. Spatial representation of sites and unidirectional conveyances of a small water

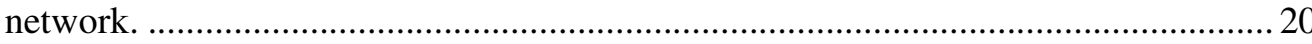

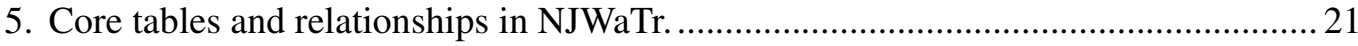

6. Screen capture showing: A hypothetical example of Conveyance data in NJWaTr.......... 23

7-18. Diagrams showing:

7. Site subject area tables, fields, and relationships...................................................... 24

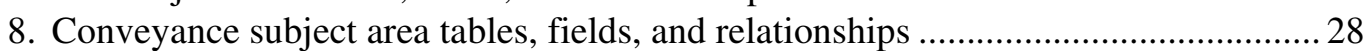

9. Transfer/Volume subject area tables, fields, and relationships. ................................ 30

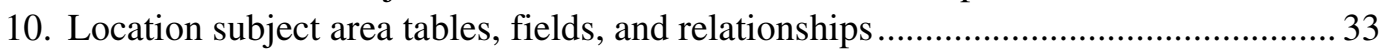

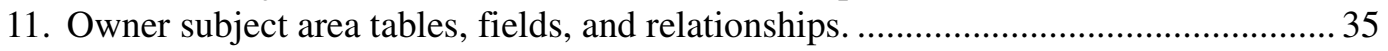

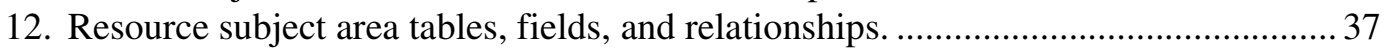

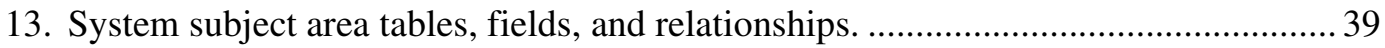

14. Address subject area tables, fields, and relationships ….............................................. 41

15. DataSource subject area tables, fields, and relationships ...................................... 42

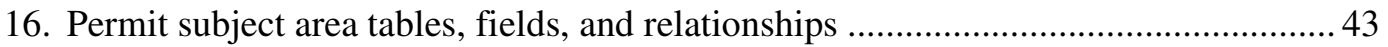

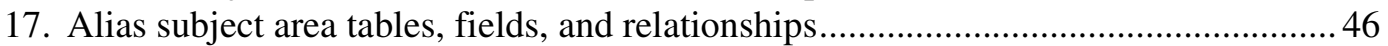

18. User-Defined Detail subject area tables, fields, and relationships............................ 48

19-22. Screen captures showing:

19. A View assembly and a virtual table focused on the tblLocation table......................65

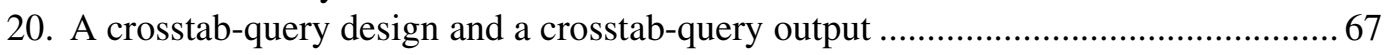

21. A complex View assembly made up of other Views. .................................................. 68

22. A virtual table made from a complex View assembly and a form based on the virtual table.

\section{TABLES}

Table 1. Table loading order and related information for the New Jersey Water-Transfer Data System (NJWaTr)

2. New Jersey Water-Transfer Data System (NJWaTr) standard preassembled views. 


\title{
Data Model and Relational Database Design for the New Jersey Water-Transfer Data System (NJWaTr)
}

\author{
By Steven Tessler
}

\begin{abstract}
The New Jersey Water-Transfer Data System (NJWaTr) is a database design for the storage and retrieval of water-use data. NJWaTr can manage data encompassing many facets of water use, including (1) the tracking of various types of water-use activities (withdrawals, returns, transfers, distributions, consumptive-use, wastewater collection, and treatment); (2) the storage of descriptions, classifications and locations of places and organizations involved in water-use activities; (3) the storage of details about measured or estimated volumes of water associated with water-use activities; and (4) the storage of information about data sources and water resources associated with water use. In NJWaTr, each water transfer occurs unidirectionally between two site objects, and the sites and conveyances form a water network. The core entities in the NJWaTr model are site, conveyance, transfer/volume, location, and owner. Other important entities include water resource (used for withdrawals and returns), data source, permit, and alias. Multiple water-exchange estimates based on different methods or data sources can be stored for individual transfers. Storage of user-defined details is accommodated for several of the main entities. Many tables contain classification terms to facilitate the detailed description of data items and can be used for routine or custom data summarization. NJWaTr accommodates single-user and aggregate-user water-use data, can be used for large or small water-network projects, and is available as a stand-alone Microsoft ${ }^{\circledR}$ Access

database. Data stored in the NJWaTr structure can be retrieved in user-defined combinations to serve visualization and analytical applications. Users can customize and extend the database, link it to other databases, or implement the design in other relational database applications.
\end{abstract}

\section{INTRODUCTION}

New Jersey's population growth and development continue to place additional demands on the State's finite water resources. Changing demographics and economic factors can increase, decrease, shift, or create new demands for water. Domestic and industrial needs typically are balanced with agricultural, recreational, wastewater discharge, and ecological uses; however, during periods of drought, sufficient water may not be available to meet all of these needs.

To better manage the State's water resources, the New Jersey Department of Environmental Protection (NJDEP) has designated twenty watershed management areas for which detailed management plans will be developed. Some of the components of the plans include water-quality assessment, land-use analysis, habitat evaluation, and water-budget analysis. The ultimate goal of this process is to develop and implement programs that will maintain or improve water quality, preserve and enhance aquatic and land habitats, and meet the water supply needs of the State.

Multiple NJDEP programs are involved with the regulation of the State's water resources. These programs include land use, water quality, water supply, and wildlife. A common issue to all of them is the amount of water available to meet the competing demands. 
To determine the amount of water that may be available in a watershed, one must understand the water dynamics taking place- the amount of rainfall, the amount and fate of surface- and ground-water withdrawals, the amount and location of surface- and ground-water discharges, the depletive and consumptive uses of water, the amount and location of water transfers between watersheds, and the amount of water that is naturally leaving the watershed as streamflow and evapotranspiration. An accounting or water-budget model is needed to establish how much ground- and surface-water might be available in a watershed at different times and under different use scenarios.

Problems encountered in doing such analyses are the collection of data and the availability of tools needed to store and manipulate the data. Many databases are in use by the NJDEP, each of which has some of the information needed to determine a water budget; none contains all the information necessary to accomplish the task.

As part of a water-use database project for the USGS Districts in New England, the New England Water-Use Data System (NEWUDS) data model and database were created to provide a tool to better organize, store, and share water-use data in the region (Tessler, 2002). To support water-budget analyses at the watershed scale for the State of New Jersey, the New Jersey Water-Transfer Data System (NJWaTr) was developed, in cooperation with the New Jersey Department of Environmental Protection, as a modification and extension of NEWUDS. The core model of both systems can store water-use information that allows water to be tracked from one point of a water-use activity, such as ground- or surface-water withdrawal, to a second point of water-use activity, such as a drinking-water service area. The links between water-use activities can start from the initial withdrawal from the resource and end with the point of final return. The "link-node" data structure present in both NEWUDS and NJWaTr has been recognized as the recommended structure for storing water-use data (Water Science and Technology Board, 2002, p 119).

NJWaTr has the following features.

- It is constructed from a conveyance-based data model rather than a site-based data model, thus promoting and encouraging a water-network approach to water-transfer and water-use data storage and investigation.

- It accommodates both single-user and aggregate water-use data in a single data model.

- It is implemented as a stand-alone and portable database in Microsoft ${ }^{\circ}$ Access (MS Access) and, therefore, is accessible to a large number of potential users. The design can be recreated in any other relational database management system with some modification.

- It can be used for large projects (state and regional studies) and small, focused projects (watershed studies).

- It is fully open to customization and extension.

Although the order of topics leads from conceptual to detailed information, some readers may prefer to read the document in a different order or to identify a section of immediate interest. The headings for the descriptive sections in the document are listed below with a brief summary of the contents.

- Development of the NJWaTr Data Model - a list of requirements that the database structure had to meet, the modeling process, and the software used. 
- NJWaTr Design Principles — conventions used in diagrams illustrating tables, fields, and relationships; normalization goals; key structures; rationale for extensive use of domain tables; naming standards; and functional table types.

- NJWaTr Data Model—definition and description of the core logical entities and relationships that serve as the conceptual foundation for the NJWaTr data model, description of tables and relationships that constitute the core of the NJWaTr database, and individual descriptions of subject areas within the design.

- Operational Issues and Procedures-items related to working with data within NJWaTr, including indexes, table loading order, supplied maintenance and update queries, and use of standardized views as templates for generalized or custom data extraction and exploration activities.

- Customizing and Extending the Data Architecture- methods recommended for adding or linking new data fields or tables to NJWaTr without compromising the database structure.

- Summary - a brief summary of NJWaTr and some advantages of using the database.

- References Cited - a list of references cited in the document.

- Appendixes-Appendix 1 contains the NJWaTr Data Dictionary, which lists and describes each table and field along with its main properties. Appendix 2 contains Domain Table Listings that show the classification and descriptive terms used to uniformly define data records within NJWaTr. The differences between the NJWaTr and NEWUDS data models are summarized in Appendix 3.

This report is intended for readers who have some background in the design or use of relational databases. Readers who are unfamiliar with data models or relational database design concepts are referred to the numerous books available on those subjects (for example, Fleming and von Halle, 1989; Hernandez, 1997; Roman, 1999) or to the Federal data modeling standard document FIPS 184 (National Institute of Standards and Technology, 1993).

Throughout the remainder of this document, logical entities are denoted by capitalization (for example, Site and Owner); table names, field names, and name parts are shown in italics (for example, the table tblSite, the field SiteTypeCategory, and the suffix _ID); example data values for fields are shown within quotes (for example, SiteTypeCategory = "treatment"); and query names are shown in boldface (for example, qrmVolumeUnitConversionFactor).

\section{Purpose and Scope}

The purpose of this document is to describe the NJWaTr data model and its physical implementation as an MS Access database. Database entities and relationships along with their components are shown in illustrations. Table loading order and preassembled views are listed in tables. The entire database design is diagrammatically represented on a plate.

This report is intended to meet the following user needs:

- to get an introduction to the NJWaTr data model;

- to evaluate the NJWaTr storage structure for use; 
- to customize a copy of NJWaTr;

- to link NJWaTr to other databases (such as geographic information systems, well- and stream-gage information, or water-quality information);

- to build software applications that service NJWaTr for data entry, data analysis, or reporting; and

- to understand the differences between the NJWaTr and NEWUDS data models.

NJWaTr is not an analytical application, but rather a design for the efficient storage, accurate retrieval, and custom manipulation of water-use data. Exploratory and analytical applications can be developed to work directly with the data in a NJWaTr database.

\section{Acknowledgments}

The author gratefully acknowledges the participation and contributions of Jeffrey L. Hoffman and Steven Domber of the New Jersey Department of Environmental Protection in the development and testing of the NJWaTr data model and database design.

\section{DEVELOPMENT OF THE NJWATR DATA MODEL}

The creation of a model that accurately describes the structure of a set of data is a precursor to any correctly designed database (Fleming and von Halle, 1989). Data modeling is the formal process of analyzing and reducing descriptions of information into separate data components, and establishing the nature and direction of relationships between those components, thereby building a structure for the data that automatically enforces the rules needed to maintain data integrity. A logical data model integrates change as part of data management by providing a generic structure that permits later extensions without affecting the validity of data already in the database. A data model also is used to create and maintain documentation of the elements that comprise the database and, thus, provides a common language and reference for all users. In addition, a data model is used as a template for implementation of a physical database design and provides a structure for data entered into the database to meet a predetermined level of detail and organization.

\section{NJWaTr Requirements}

A set of data and user requirements is used to clarify the use of, and information contained in, the database, to guide the development of the database structure, and to serve as final checkpoints for the end product. The general requirements for the NJWaTr database design are

- Store data that will facilitate summarization of withdrawals and water-use activities in New Jersey (such as in Hoffman and Lieberman, 2000),

- Allow for the storage and retrieval of information on single-user and aggregate water use,

- Store classification information at multiple levels on the uses and suppliers/users of water to allow for flexibility in comparisons and aggregations, 
- Allow data to be summarized in different spatial contexts by storing location information by state, county, municipality, hydrologic unit, water region and management area, critical area and zone for regulated aquifers, and drought region,

- Allow area (non-point) locations to be explicitly partitioned as location-percentages that span various geopolitical and hydrologic boundaries in order to distribute the transfer volumes among the areas,

- Allow for the storage of data on water exchange (volume per unit time) in a variety of measurement and reporting units that cover a range of transfer time scales (for example, daily, monthly, or annual data) and facilitate the conversion of the data to common units of measurement so that data are retrieved in uniform units,

- Allow for the storage of alternative estimates of individual water transfers that are based on different determination methods or data sources,

- Allow sites and owners to be associated with and identified by their regulatory permits,

- Allow for alternative identification and naming schemes (aliases) for the main objects listed in the database,

- Identify sources of data and allow contact information to be stored, and

- Allow optional, user-defined details to be added for major objects in the database.

\section{The Modeling Process}

NJWaTr is a modification of the NEWUDS design (Tessler, 2002). Development of the NJWaTr database began with a review of the functional aspects, strengths, and limitations of the NEWUDS data model. This was followed by detailed discussions about tracking of water transfers and water use in New Jersey. A conceptual model was developed to meet the data-storage goals in New Jersey; the model includes items such as water resources (rivers, canals, ponds, reservoirs, and aquifers), water uses, water purveyors, single and aggregate water-users, and regulatory identifiers (permits and other codes) that were identified in the discussions. The major data objects were identified, consolidated where appropriate, and represented in the conceptual model as entities with defined relationships. The existing NEWUDS data model then was evaluated, and it was determined that NEWUDS could be modified to meet the needs of a relational database for New Jersey. Modifications include renaming, redefining, dropping, and adding entities and attributes. When a general concept could be realized in more than one data structure, the alternatives were presented; the implications of each were discussed, and the contextually accurate structure was determined and incorporated into the model. Exceptions to data generalizations and required/optional data elements were identified, and the resulting model was evaluated and revised. This process continued in an iterative fashion until all the information elements identified in the NJWaTr requirements were set in the data structure, and the factual statements represented by relationships between data objects were accepted.

Some data structures that did not exist in the NEWUDS design were modeled in NJWaTr in a syntactically correct manner but had to be tested to determine whether they could perform their intended functions for data manipulation and information retrieval. Use cases were developed for proof-of-concept testing of these new data structures. When results of the tests confirmed the structures were valid for their purposes, the structures were discussed and incorporated into the model. 
Design diagrams and listings of table/field properties (data dictionaries) were used during discussions of the model and reviewed between meetings. NJDEP personnel participated in the design phase to ensure that the database contained those categorical and classification terms needed to store, describe, validate, list, summarize, and aggregate water-tracking information. A guiding principle was that the data needed to provide answers to what, where, how, and when questions used in water-use analysis should be in a form that is familiar to the user, in terms that are well defined and commonly used.

\section{Modeling Software}

The NJWaTr database was designed using the data-modeling tool ERwin version 3.5.2 (Computer Associates, Inc). Data-modeling tools facilitate rapid design and modification, and invisibly handle many details of the data structures that can affect the quality and integrity of data. ERwin can be used to model and generate physical databases for a number of relational database management systems. For MS Access, ERwin provided editing capabilities for application-specific data properties through a physical model based on the logical design. After the NJWaTr data model was completed, ERwin was used to create the physical implementation of the database in MS Access by providing table and field identities, discrete properties (field type, size, and default values), relationships, and all table and field constraints that ensure the integrity of keys and data.

\section{NJWaTr DESIGN PRINCIPLES}

The process of building a database and communicating its features is enhanced by establishing certain rules of construction and standards for uniformity. Design principles provide standardized rules for creating and assembling the database and for facilitating communication about how different elements of the database function together. This section describes the method used for the visualization of database objects, the goals for table construction and establishing formal relationships, the rationale for extensive use of domain tables, and the naming standards applied to tables and fields.

\section{Entity/Relationship Diagramming Conventions}

Entity/Relationship (E/R) diagrams are used to visualize database designs, and these diagrams are presented throughout this report to help describe the NJWaTr structure. Several different display and notation methods are commonly used for E/R diagrams, and all allow graphical/text representation of entities, relationships, and attributes. The diagrams in this document use the Integration Definition for Information Modeling (IDEF1X) standard as defined in National Institute of Standards and Technology (1993) and as implemented in the ERwin modeling software. An E/R diagram showing the complete set of relationships between Owners and Addresses illustrates several diagramming conventions (fig. 1).

In the $\mathrm{E} / \mathrm{R}$ diagram, a box is used to denote an entity, which equates to a single table in the physical database. The table name appears above each entity box. One or more entity attributes, which equate to fields in the physical database, are listed within the box. Lines connecting entities represent the defined relationships. Lines signify that the entities share a key field and can be linked in the physical database through the relationship.

Each attribute within a table is shown with its assigned data type for the MS Access implementation of NJWaTr. A primary key (PK) is the field or group of fields that uniquely identify a record in the table. The PK for each entity (composed of one or more attributes) is listed at the top of the attribute list within 


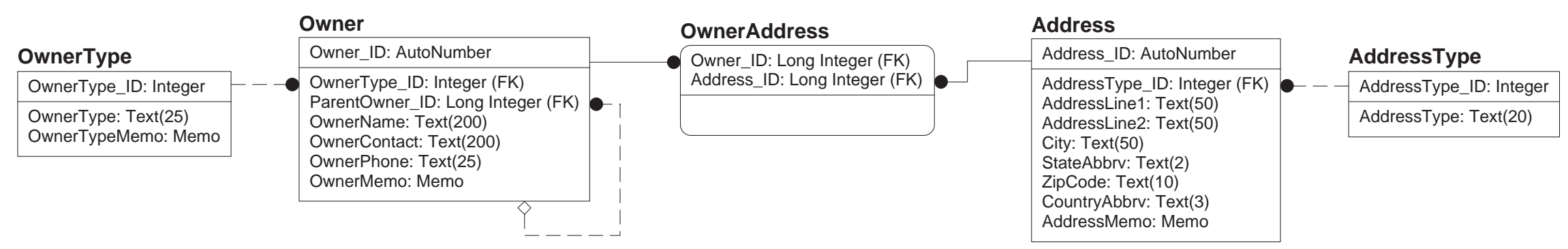

\section{EXPLANATION}

Table and Relationship Symbols

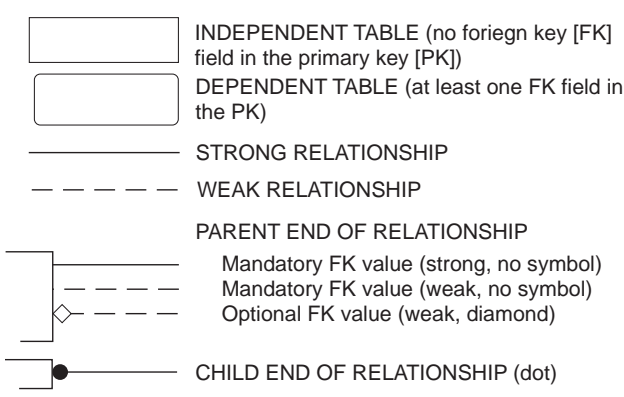

\section{Field Property Indicators}

PK PRIMARY KEY (PK) FIELDS ABOVE LINE

NON-PK FIELDS BELOW LINE

(FK) FOREIGN KEY FIELD

(20) NUMBERS IN PARENTHESES

INDICATE TEXT FIELD

MAXIMUM NUMBER OF

CHARACTERS

Figure 1. Graphical representation of entities, attributes, and relationships (E/R diagram). 
the entity box and is separated from the non-PK attributes by a horizontal line. When a primary key from one table (the parent) is used in another table (the child) to create a defined relationship, the field is called a foreign key in the child table and is designated by FK in the diagram.

If a FK field also is part of the identifying PK in the child table, the relationship is said to be strong, and the relationship line is solid in the diagram (fig. 1). If the FK field is a non-PK (descriptive) attribute of the child table, the relationship is said to be weak, and the line is dashed. Both strong and weak relationships are illustrated in figure 1. To facilitate visualization of table dependencies, a table that is dependent upon another through a strong relationship (a FK field is part of the PK) is shown with rounded corners, whereas tables that do not have FK dependencies in their PK (independent tables) are shown with squared corners. Strong relationships pair Owners with Addresses in the table OwnerAddress in figure 1 (the incoming FK fields also are PK fields in the child table). Weak relationships are represented where the OwnerType and AddressType tables provide descriptive, non-PK attributes to the tables Owner and Address, respectively.

Each relationship line has a direction and a cardinality. The direction is indicated by the presence of symbols at the parent and child ends of the relationship line. The parent entity in the relationship is represented by no marking on the relationship line or a hollow diamond. The child entity in the relationship is shown by a solid circle. Direction symbols in an E/R diagram make it easier to visualize the flow of information in a data structure and are especially useful for identifying parent/child entities in a PK/FK relationship in which one table provides attributes to another.

Cardinality describes how each record in one entity relates to a count of records in another entity, and cardinality is denoted on diagrams by the kind of symbols on the ends of relationship lines. There are many possible relationship cardinality variants. In a one-to-one (1:1) relationship, each record in the parent entity has exactly one match in the child entity. In a one-to-many (1:n) relationship, each record in the parent entity has one or more matching records in the child entity. In the NJWaTr model, all but two of the relationships are one-to-zero-one-or-more (1:0,n). These relationships are indicated by a solid or dashed line with no symbol on the parent end and a solid circle on the child-end. These relationships, which are illustrated in figure 1, are used when each parent can have zero, one, or more children, but each child must have a parent (the FK cannot be unassigned or Null). Thus, each OwnerType (for example, "Municipality") can serve zero, one, or more than one Owner, and each Owner must have an assigned OwnerType. The zero part of the relationship cardinality allows an OwnerType record to exist in the domain without actually being used by an Owner until needed and is very useful for filling a table with a list of all permissible values before other data are entered into a database.

The other cardinality in NJWaTr is shown in figure 1 as the self-join for Owner. Each Owner record can point to another Owner as its parent by storing the parent's Owner_ID in the ParentOwner_ID field. The hollow diamond on the parent-end of the self-join relationship line means that the relationship is optional (FK may be Null); that is, an Owner need not have a designated Parent Owner.

\section{Normalization}

Normalization refers to the process of structuring a database to avoid data redundancy and to reduce complex data elements to their component parts as separate tables and relationships (Fleming and von Halle, 1989, p. 179). The NJWaTr model is structured to approximately third normal form. This means that each table stores information dependent upon a single key; each datum or descriptive element is recorded in only one location; and keys to those data are carefully dispersed among other tables as surrogates for data in the parent table. Because a normalized design often appears to be rather complex and cumbersome to manipulate, queries are constructed that aggregate data from related tables in a denormalized table view. This fa- 
cilitates data review, manipulation, and reporting, while the actual data structure is hidden from the user. (The construction of denormalized views is discussed in the section, "Construction and Use of Views.")

\section{Keys and Relationships}

Each table in the database is designed to hold a specific, defined, and delimited set of data. Each record in a table (a row or the particular collection of data it represents) is identified by a unique PK value, a number. The PK and its value in one table (the parent) can be used in another table (the child) as a surrogate for all the information it represents in a specific row of the source table. In the relationship, the PK from the parent table is called a foreign key in the child table. The relationship established between tables through the shared PK/FK fields provides a pathway for the data to be combined into a single view of the records from both tables. With many tables related to one another in various combinations, the relational model provides a way to extract data from any two or more tables in any combination desired, if an unambiguous pathway of relationships exists between them.

The MS Access database engine protects PK fields in two ways: (1) fields identified as the PK must have a value whenever new rows are added to a table, and (2) MS Access prevents the PK values from being duplicated in other rows of the table (each PK value is unique within a table). Most tables in NJWaTr have a single field that serves as the PK; the PK value itself is an incrementally assigned integer that represents a row of data in the table. Exceptions are the association tables, described farther on, where a single PK field is not practical or desirable. Thus, information-rich keys (fields that have apparently unique values, such as a code name) that could serve to identify data rows are not allowed to be PK fields but are relegated instead to attributes identified by an information-neutral numeric key. The possibility that an information-rich key value could be reassigned in practice and, thus, corrupt associated data in other tables was reason enough to prohibit their use as PKs in the NJWaTr model.

In some tables, a complex key consisting of two or more fields could serve as the PK (for example, the combination of a town code and a state code); however, the convention used in NJWaTr was to relate tables by providing a single field to serve as a surrogate for any such complex PK (association tables excepted). The design was standardized to use a single field as a PK even when other information fields, alone or in combination, would serve the same purpose. For NJWaTr, storage of a surrogate PK does not add significantly to the size or complexity of the database, and the surrogate key is more convenient to use than multi-valued, complex keys carried through relationships between tables. (For additional information about the advantages of using surrogate keys, see Lonigro, 1997.) For added protection against duplication of identifying information in non-PK fields within tables, an alternate unique index may be created in a table. (See the description of NJWaTr indexes in the "Indexes" section.)

Relationships between tables are built into the database. The relationship properties, once defined, are enforced by the MS Access database engine. To protect the integrity of the keys across tables, referential integrity constraints are placed on the keys through the relationship. All relationships in NJWaTr have a set of common settings for protection of the keys and the data they represent.

- All tables have a PK consisting of one or more fields; each record in each table is identified by a unique key value.

- All non-recursive FK fields are required to hold a value for each record, and the FKs must take a value from the collection of parent PK values.

- Changes to the value of a PK are cascaded to child records; all occurrences of the PK value in the related 
FK in other tables are updated automatically.

- Record deletion is restricted if the PK value also exists as a FK value in another table. MS Access displays a warning when children of the record (holding that FK value) are in other tables and prevents related records from becoming orphans.

These rules, along with enforcement of the uniqueness of PK values, provide the basic protection needed to prevent key corruption and to maintain table and referential integrity throughout the database. Relationship rules can be adjusted later as needed after experience is gained with NJWaTr.

\section{Domain Tables}

In NJWaTr, lists are used extensively for classification or description of items in the database. Lists that serve the main data tables and each other constitute 41 of the 76 tables in NJWaTr (54 percent). These tables are called domain tables because each provides specific information describing a particular subject domain. In practice, domain tables contain the set of permissible values from which actual values are taken. An example of a domain table is $t d s$ State, in which each record is for an individual state, and the fields provide information on the state (for example, name, postal abbreviation, and code number). A relationship with a domain table can, therefore, provide many different pieces of information through the single value of the inherited key field. Domain tables significantly enhance sorting and grouping options for the related data by providing discrete tables in which such actions can be initiated; they also facilitate maintenance and periodic evaluation of acceptable values for a subject domain.

The value of the many separate domain tables needs to be emphasized because it is possible to store domain data as fields in the tables they serve. Normalization rules dictate that data must be compartmentalized as much as possible to prevent redundancy, which is achieved in NJWaTr by using discrete domain tables. Domain tables provide considerable power to NJWaTr because they can be extended as needed by adding to the list and by adding new descriptor fields without affecting the rest of the database design.

In addition to acting as lookup tables, domains are used to create larger domain structures in NJWaTr through clustering and nesting. A domain cluster consists of two or more unrelated domain tables joined together in a base table to establish a valid combination of domain values; the base table can have additional attributes that describe the properties of each specific combination. Clusters of domain tables can be used to assemble complex sets of descriptive or hierarchical information from a limited set of choices (individual domains), and the result is carried through to a single key value. A domain cluster example in NJWaTr is the VolumeUnit entity (described in the "Transfer/Volume Subject Area" section) where two domains are combined in a single table to provide any desired combination of decimal and quantity terms in a controlled fashion.

Nesting of domains is implemented in NJWaTr where hierarchies of descriptive terms are maintained as separate lists with enforced parent-child relationships. For example, states, counties and minor civil divisions (MCDs), which roughly correspond to towns, are represented as nested domain tables that serve the Location entity for Sites. In an application, nesting would be implemented as the ability to look up a state to reveal its counties and the counties' MCDs, or to look up an MCD to reveal its parent county and state. In a nested domain structure, the table that contains the smallest-scale descriptive term (MCD in the example above) is usually the one that is linked to a data table. This allows any of the larger-scale levels to be retrieved through the nesting relationships to describe the data object they serve. 


\section{Naming Standards}

Naming standards convey important information about an item's identity, contents, or both with little more than an understanding of the convention used. In the NJWaTr database, naming standards apply to both tables and fields.

\section{Table- and Field-Name Construction}

The four general name-construction standards are listed here with explanations.

1. Names are constructed using whole words (with a few exceptions) to provide as much intuitive meaning as possible.

2. For names that are composed of word phrases, the first letter of each word in the phrase is capitalized to assist in reading the name.

Examples of phrased field names include ConveyanceDetailLabel, ConversionToMillion, TimeInterval, and TransferEffectiveDate. Names that do not consist of whole words are constructed using a commonly accepted acronym or abbreviation, specifically MCD (Minor Civil Division of the U.S. Census Bureau), HUC (Hydrologic Unit Code), NJ (New Jersey), and MG (million gallons, the common unit used for a water-transfer volume). The only other exceptions to the use of whole words in names are Abbrv for Abbreviation, Det for Determination, and Mgmnt for Management. The abbreviations help limit the size of item names without affecting their readability, specifically StateAbbrv, CountryAbbrv, VolumeUnitAbbrv, LocationDetMethod, and WaterMgmntArea.

3. Names are constructed only of alphabetic characters; no numerals, punctuation, or other special characters (such as spaces or dashes) are allowed, except for the underscore in singular PK fields that consist of the table root name and an _ID suffix.

The exception here is an important rule of its own: the PK field of each non-associative table is identified by the table root name and a suffix of_ID, such as Location_ID as the PK field of the tblLocation table. This standard of using an_ID suffix for a key field allows distinction between key and non-key (data) fields. The presence of _ID keys as FK fields in a table also clearly indicates that one or more non-key fields are available in a related table of the same name.

In NJWaTr, six field name variants represent three special cases for the _ID rule. The first case involves recursion within a table where a field has an _ID suffix and points to another record in the same table. The recursion keys in NJWaTr are ParentOwner_ID and ParentSystem_ID. Use of these fields allows a nested hierarchy to be represented by holding a value pointing to the PK of another record in the same table (ParentOwner_ID to Owner_ID and ParentSystem_ID to System_ID). The leading word Parent in the field name identifies these recursion keys. Use of these fields is optional so that top-of-hierarchy items or items without a designated Parent are not required to hold a value.

In the second case, a component structure is defined by pairing a parent record with one or more child records from the same table. This is represented in the table tadResourceStructure where a ParentResource_ID and a ChildResource_ID are paired, each pointing to different Resource records in the tblResource table. The Resource record identified through the ParentResource_ID is a composite of more than one Resource, and the ChildResource_ID values associated with it indicate the other Resources that 
make up that composite. The tadResourceStructure table will be discussed in the section, "Resource Subject Area."

In the third case, two fields use combined name forms, SiteTypeFromID and SiteTypeToID. These fields point to a key field in a domain (SiteType_ID) but lack a direct, defined relationship between the tables. These fields are used as data rather than keys (PK or FK). Thus, the underscore must be omitted. The table maintenance function of those semi-key data fields is discussed in the section, "Conveyance Action Phrase Updates."

4. Fields with a Yes/No data type begin with the word "Is," those with a DateTime data type end with the word "Date," and those with a Memo data type end with the word "Memo."

Fields with a Yes/No (Boolean), DateTime, or Memo data type are unlikely to need modification in a working implementation of the NJWaTr database, so coding the data type into the name provides information about the nature of the field. Coding the data types of all other fields with a suffix or prefix was not considered practical because the data types or sizes of some fields may need to be changed as experience grows with use of the database. For example, a Text field may need to be converted to a Memo field if the storage required by the field exceeds 256 characters, or a single precision number may need to be converted to double precision to accommodate unanticipated but valid values for a field.

Seven Yes/No fields are present in NJWaTr: IsPrimaryMCD, IsPrimaryCounty, IsPrimaryState, IsPrimaryHUC, IsPrimaryResource, IsDefaultVolume, and IsNumericDetail. They are named as a question phrase to emphasize the Yes/No nature of the value they hold for each data record.

Eight DateTime fields are present in NJWaTr: ExpectedFractionEffectiveDate, ExpectedFractionEndingDate, ResourceDetailEffectiveDate, ResourceDetailEndingDate, SiteDetailEffectiveDate, SiteDetailEndingDate, TransferEffectiveDate, and TransferEndingDate. Each of these fields is used to set a bounding date on a data record.

Thirty Memo fields are present in NJWaTr, and all but one use the word Memo as a suffix. Memo fields are optional, do not have size limitations, and are used to store remarks or notes about a record in a table. The one Memo field that does not use the Memo word suffix is SiteContact. The table tblSite contains a SiteMemo field for general notes about a Site record, whereas the SiteContact field stores variable amounts of contact information for a Site record and cannot be limited to the 256 characters of a MS Access text field.

\section{Table Functional Prefixes}

For MS Access databases, table prefixes in wide use today are either $t b l$ or $t l k p$, where the latter refers to lookup (domain) tables and the former to all other tables. NJWaTr contains 76 individual tables, and more than 2 functional table types are recognized. To facilitate rapid identification and communication of the function of different tables in the database, five functionally distinct types of tables are defined by a threeletter prefix applied to the table name (fig. 2). Table names are, therefore, distinguishable from field names because all table names have lower case prefixes, and fields do not. In addition, each of the five table types is displayed in a different color in diagrams of the database structure, imparting significant and immediate understanding of the functional relationships of data elements and relational structures. The five table name prefixes used in NJWaTr and their definitions are provided below. 


\begin{tabular}{|c|c|}
\hline Functional table prefix & Diagram display format \\
\hline \multirow{2}{*}{ tbl - basic data table } & tblConveyance \\
\hline & Conveyance_ID \\
\hline $\begin{array}{c}\text { yellow display color, } \\
\text { square corners }\end{array}$ & $\begin{array}{l}\text { ConveyanceType_ID (FK) } \\
\text { ConveyanceAction_ID (FK) } \\
\text { ConveyanceName } \\
\text { ConveyanceMemo }\end{array}$ \\
\hline \multirow[b]{3}{*}{$\begin{array}{l}\text { blue display color, } \\
\text { square corners }\end{array}$} & tdsState \\
\hline & State_ID \\
\hline & $\begin{array}{l}\text { CountryAbbrv } \\
\text { StateCode } \\
\text { StateAbbrv } \\
\text { StateName } \\
\text { StateLatitude } \\
\text { StateLongitude }\end{array}$ \\
\hline \multirow{3}{*}{$t d x-$ domain, user-extendable } & tdxStaff \\
\hline & Staff_ID \\
\hline & $\begin{array}{l}\text { StaffInitials } \\
\text { StaffName } \\
\text { StaffAffiliation } \\
\text { StaffMemo }\end{array}$ \\
\hline tas - association, simple & tasOwnerAddress \\
\hline white display color, & $\begin{array}{l}\text { Owner_ID (FK) } \\
\text { Address_ID (FK) }\end{array}$ \\
\hline rounded corners & \\
\hline tad - association, with data & tadLocationHUC \\
\hline green display color, & $\begin{array}{l}\text { Location_ID (FK) } \\
\text { HUC_ID (FK) }\end{array}$ \\
\hline rounded corners & $\begin{array}{l}\text { HUCFraction } \\
\text { IsPrimaryHUC }\end{array}$ \\
\hline
\end{tabular}

Figure 2. Examples of tables with functional prefixes and their diagram display formats. 
$t b l$-basic data table; for example, tblConveyance. Basic data tables hold primary data entered into the database and include non-associative core entities. Typically these tables are the focus of denormalization in the creation of multi-table views (discussed in the section, "Construction and Use of Views"). The $t b l$ tables have a single PK field (_ID suffix) that is an incrementally assigned integer for each new record (an AutoNumber field) and is guaranteed by MS Access to be unique. These tables always contain one or more fields that are FKs from domain tables, and thus $t b l$ tables inherit classification and descriptive terms as extended attributes. The _ID keys from $t b l$ tables are dispersed as FK fields among other tables in the database. The $10 \mathrm{tbl}$ tables in NJWaTr (13 percent of the tables) are tblAddress, tblAlias, tblConveyance, tblLocation, tblOwner, tblPermit, tblResource, tblSite, tblTransfer, and tblVolume. These tbl tables are shown as yellow rectangles in the figures in this report.

The next two functional table types deal with domains and have a prefix beginning with the letters $t d$. These tables are used to provide list information for selective use in other tables. Domain tables also are sometimes known as lookup or reference tables, and other naming conventions in the database industry may use the tlkp prefix.

$t d s$-domain table, static; for example, tdsState. Static domain tables hold a list of classification or descriptive items that are used by other tables. The list of items is considered static because it is pre-populated and expected to be complete (for example, the $t d s$ State table is fully populated with records that describe New Jersey and neighboring states). The $t d s$ tables have a single PK field (_ID suffix) that is an integer; to prevent inadvertent additions to the static list, the key value must be manually entered whenever new entries are made. Manual key entry also allows the creation of custom unique key values for use with the choice list so that the table is not limited to automatically generated numbers (for example, to add a " 0 " key value as a default selection that represents an unknown condition). The $t d s$ tables typically serve one or two receiving tables; therefore,_ID keys in $t d s$ tables are found in other tables as a FK. The $26 t d s$ tables in NJWaTr (34 percent of the tables) are tdsAddressType, tdsConveyanceAction, $t d s$ ConveyanceActionCategory, $t d s$ ConveyanceType, $t d s$ County, tdsCriticalArea, tdsCriticalZone, $t d s$ DroughtRegion, tdsHUC, tdsLocationScale, tdsMCD, tdsOwnerType, tdsPermitSeries, tdsResourceType, tdsSiteType, tdsSiteTypeCategory, tdsSiteTypeSubcategory, tdsState, tdsTimeInterval, tdsUseGroup, tdsUseType, tdsVolumeUnitDecimal, tdsVolumeUnitQuantity, tdsWaterBodyType, tdsWaterMgmntArea, and $t d s$ WaterRegion. These $t d s$ tables are shown as blue rectangles in the figures in this report.

$t d x$-domain table, user-extendable; for example, $t d x$ Staff. User-extendable domain tables provide a list that the user can add to as needed. These tables, therefore, differ in an important way from $t d s$ tables. The $t d x$ tables are considered incomplete at the outset, and cannot be populated fully before the database is used. For example, new entries will be made to the $t d x$ Staff table whenever new staff members need to be identified in the database. The $t d x$ tables have a single PK field ( $I D$ suffix) that is an AutoNumber field (incrementally assigned integer) to facilitate additional entries without regard to key value assignment. Although similar in construction to $t b l$ tables, $t d x$ tables provide an extendable domain list that is used to characterize records in data tables, rather than to hold primary data for the database. The 15 $t d x$ tables in NJWaTr (20 percent of the tables) are $t d x$ AliasLabel, tdxConveyanceDetailLabel, tdxDataSource, tdxLocationDetMethod, tdxPermitLabel, tdxResourceDetailLabel, tdxSiteDetailCategory, tdxSiteDetailLabel, tdxStaff, tdxSystem, tdxSystemType, tdxVolumeDetailLabel, tdxVolumeMethod, tdxVolumeMethodCategory, and tdxVolumeUnit. These $t d x$ tables are shown as gray rectangles in the figures in this report. 
The final two functional table types are associative and have a prefix beginning with the letters $t a$. These tables differ from other types in that they are dependent on at least one other table by having all or part of their PK consist of FK fields; therefore, records in these tables cannot exist without the presence of a source record in a parent table. Association tables serve the functions of (1) resolving potential many-tomany relationships between two or more tables, (2) describing a property relating to a specific combination of records from other tables, or (3) describing a property of a parent table that is defined or constrained in some way by another attribute, such as a time interval. These tables also are termination or reflexive points in the data flow within a relational design in that they have incoming but not outgoing relationships; the PKs of these tables are not passed on as FKs to any other tables. Association tables also are sometimes known as junction or intersection tables.

tas-association table, simple; for example, tasOwnerAddress. Simple association tables are used to resolve many-to-many relationships between two or more tables and are composed only of FK fields that all participate in the PK; that is, each field in the table is part of the PK and also a FK that points to a record in another table. For example, use of the association table tasOwnerAddress allows an Owner to have more than one Address, and a single Address can serve more than one Owner. The tas association table name is a combination of the two or more parent table names that share the association. (In this case, records from tables tblOwner and tblAddress are paired in the table tasOwnerAddress.) The tas tables have a complex PK composed solely of the FKs from each table involved in the association (in this case, Owner_ID and Address_ID); no other fields are present in tas tables. MS Access maintains the integrity of the association by allowing each combination only once, thus ensuring the primacy of the key. The PK values in tas association tables can be entered directly to limit a list to valid combinations or can be assembled as needed by deliberately pairing records from the parent tables in the desired combinations. The 11 tas tables in NJWaTr (14 percent of the tables) are tasConveyanceAlias, tas ConveyanceOwner, tasOwnerAddress, tasOwnerPermit, tasResourceAlias, tasSiteAddress, tasSiteAlias, tasSitePermit, tasSiteTypeDetailLabel, tasSystemSite, and tasWaterBodyDetailLabel. These tas tables are shown as white rectangles with rounded edges in the figures in this report.

tad-association table, with data; for example, tadLocationHUC. As used here, tad association tables with data represent a collection of functional table types that share two identifying characteristics. (1) They are dependent tables with at least one field in the PK that is a FK from another table. (2) They have at least one field that is not part of the PK. Some tad tables also may contain a non-FK field in the PK, such as a time constraint. The $t a d$ association table name either is a phrased combination of the two or more parent tables similar to tas tables (for example, tadLocationHUC) or is a phrase that begins with the root name of the main table being served plus one or more descriptor terms (for example, tadResourceDetail). The 14 tad tables in NJWaTr fall into four groups.

In their simplest form, tad tables are similar to tas tables in that they pair records from two or more tables to form a PK that consists solely of FK fields, but they also store data that describe some property unique to the association itself (that is, to the combination of values that form the PK). For example, tadLocationHUC combines a Location_ID with a HUC_ID (identifying a specific hydrologic unit or watershed) to form its PK; like tas association tables, this allows individual Locations (for example, a county-level aggregate location) to be associated with more than one HUC and each HUC to be associated with more than one Location. Unlike a tas table, however, the tadLocationHUC table also adds two non-key fields, HUCFraction, which stores the fractional part of the Location represented within the paired HUC, and IsPrimaryHUC, which defines the individual pairing of key values that represent the HUC that should be always be retrieved with the Location whether or not more than one HUC is associated. Tables with this structure are tadConveyanceDetail, tadLocationCounty, tadLocationHUC, tadLocationMCD, tadLocationState, tadSiteUseType, and tadVolumeDetail. 
A second form of tad table also contains FKs from two or more tables but includes a non-FK field as part of the primary key to provide an important constraint on the association. Three tables are in this group: tadResourceDetail, tadSiteConveyance, and tadSiteDetail. In the tables tadResourceDetail and tadSiteDetail, the three-part PK consists of a main parent entity _ID, a domain Label_ID, and an EffectiveDate date/time field. Adding the temporal field to the PK allows a main parent entity record to be paired with the same Label multiple times to show how an attribute specific to the association changes over time, and therefore, a history of values can be maintained. The third table in this tad group is tadSiteConveyance in which two Sites need to be defined as the endpoints of a unidirectional Conveyance. The PK consists of the FK field Conveyance_ID and the non-FK field FromOrTo that can hold only two possible values, "From" or "To." Thus, the PK ensures that each Conveyance can have only two records in the table to represent the separate end points as Conveyance_ID + "From" and Conveyance_ID + "To." The final field in the tadSiteConveyance table is the Site_ID, which is a FK field but does not participate in the PK; it is the attribute that identifies the individual Site at the From or To end of a Conveyance.

A third form of tad table is represented by the three tables tadUseConsumedFraction, tadConveyanceExpectedFraction, and tadResourceStructure. These tables receive their FK field(s) from only one parent table and essentially "hang" additional, conditional, descriptive attributes off the parent table. The table tadUseConsumedFraction pairs a UseType_ID with a Month to store a static numeric value applicable to a particular month of the year for a UseType. The table tadConveyanceExpectedFraction uses a PK that combines a Conveyance_ID with an ExpectedFractionEffectiveDate field to allow multiple, time-bounded fraction values to be stored for a Conveyance. The table tadResourceStructure has a PK that consists of two FKs, ParentResource_ID and ChildResource_ID, both of which represent Resource_ID selections that define the parent and child relationships of composite water Resources, along with a fractional value unique to each combination of parent/child.

The last tad table, tadSiteResource, is critical to defining the relationship between those Sites involved in withdrawals or returns and their associated Resources. This dependent table has only the FK field Site_ID as the PK and will hold only records for Sites that interact with Resources. In addition to the single PK field, non-PK fields in this table are the FK Resource_ID, an integer field ConnectionCount, and two domain FKs CriticalArea_ID and CriticalZone_ID characterizing well locations in regulated aquifers (a FK value for "not applicable" is assigned to Sites where those domains do not apply). By keeping Site_ID as the sole PK field, each Site record is constrained to be associated with only one Resource record, and the additional properties that describe the Site-Resource pairing also can be stored. Because only Sites that interact directly with a Resource can have a record in this table, the structure is sometimes referred to as a subtype (Fleming and von Halle, 1989). In NJWaTr, the tadSiteResource table functions to store a dependent Site-Resource association along with properties specific to each pairing, so it is included with the other tad tables.

To summarize, tad tables represent a collection of dependent tables that contain non-FK fields. They are used to resolve potential many-to-many relationships along with some property unique to the association or to describe a value for records from one or a combination of tables that is constrained in some way. Further study of a variety of data models should help refine the recognition and definition of the functional table types represented here by tad tables, but for now they are handled as a composite group with a single prefix and diagram color. The $14 \mathrm{tad}$ tables in NJWaTr (18 percent of the tables) are tadConveyanceDetail, tadConveyanceExpectedFraction, tadLocationCounty, tadLocationHUC, tadLocationMCD, tadLocationState, tadResourceDetail, tadResourceStructure, tadSiteConveyance, tadSiteDetail, tadSiteResource, tadSiteUseType, tadUseConsumedFraction, and tadVolumeDetail. These tad tables are shown as green rectangles with rounded edges in the figures in this report. 
Recognizing and understanding the naming conventions described above can be useful in exploring the NJWaTr database. New fields or accessory tables created to extend the functionality of the database should follow the naming conventions to ensure uniform communication about the identity and functional properties of the new items. Refer to section "Customizing and Extending the Data Architecture" for more information on how this can be accomplished.

\section{NJWaTr DATA MODEL}

The full structure of the NJWaTr logical model and physical design is presented in this section. First, the core entities and their relationships are defined and described. The model then is broken into subject areas centered on specific entities. Detailed explanations are provided, and new related entities are introduced at the submodel scale. The general use of addresses, data sources, permits, aliases, and user-defined details for different subject areas is discussed in the last five parts of this section.

NJWaTr contains 76 tables, 95 defined relationships, and 311 fields. The complete structure is shown in plate 1. Forty-one of the 76 tables in the NJWaTr database are domain tables (54 percent), 25 are association tables ( 33 percent), and 10 are basic data tables (13 percent). The dominance of domains in the distribution of non-key fields in tables is similar: of 160 non-key (data) fields in the database, 99 (62 percent) are in the domain tables, including 15 Memo fields for notes about domain entries. The extensive use of domain tables provides flexible classification of the data elements, and the tables serve as the primary objects for grouping, sorting, filtering, and summarizing information.

Appendixes 1 and 2 provide the complete NJWaTr data dictionary and lists of the values represented by the domain tables, respectively. The data dictionary provides definitions for each table and field in a tabular format. Readers can refer to those pages for additional details not given below.

\section{Definition of the NJWaTr Core Entities}

The core of the NJWaTr data model can be illustrated by the following statements and conceptual diagrams. More details for each entity are given farther on in this document.

Each object that can be named as a source or target of water movement is called a Site. A Site can be a discrete object such as a well, water-treatment plant, or commercial business, or an object with more than one component represented by a single Site name (for example, a purveyor service area). Sites are categorized by types and have other definable characteristics. Any two Sites that exchange water are joined by a unidirectional Conveyance, which can be an actual pipe, conduit, or aqueduct, or a virtual representation of the connection between the Sites. A single Site can serve as the input or output end for multiple Conveyances. Together, all Sites and their Conveyances form a water network. This is equivalent to the "link-node" data structure recommended for storing water-use data (Water Science and Technology Board, 2002, p 119).

A Transfer/Volume is a record of a single water-movement estimate reported from or calculated for a specific Conveyance between two Sites over a specified time interval. Each Transfer/Volume is characterized at minimum by a volume value for the water exchange, a unit of measurement, the time interval covered by the estimate, and the source and method of the estimate. The full history of Transfer/Volume values can be stored for all Conveyances and the Sites they connect. A representation of estimated daily Transfer/Volume values for the Conveyance between a well and a treatment plant, as measured and reported by the treatment plant operator, is shown in figure 3. 


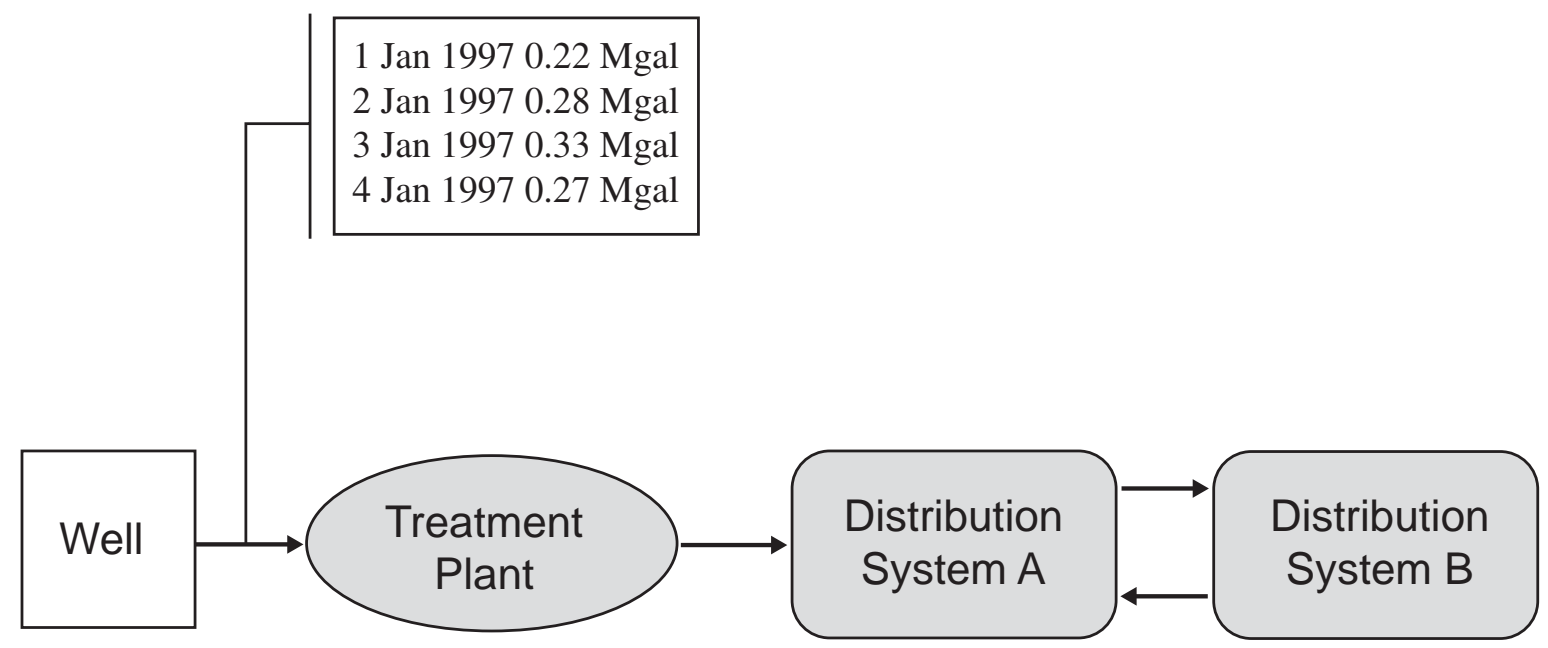

Figure 3. Representation of a small water network consisting of a withdrawal well, a treatment plant, and two distribution systems, along with their unidirectional conveyances and four estimated daily transfer/volume values for one conveyance. 
Two additional entities complete the core of the data model. The spatial Location of each Site is defined by a scale term (point or specified area) and latitude and longitude coordinates (actual point or centroid); realistically, more than one Site can share the same Location. Location information provides a spatial reference and visualization potential for the water network. Each Location also links to a hierarchy of other spatial attributes (town, county, state, hydrologic unit, and others). A spatial representation of an example water network is shown in figure 4. In this network a withdrawal well and treatment plant share a single point Location (parts of a single facility), and two town distribution systems (each an additional Site in the data model) are located at town centroid positions. The reciprocal exchange of water between the two distribution systems is indicated by two unidirectional Conveyances.

An Owner controls and maintains Sites and Conveyances and can serve as the source of the Transfer data. Each Site and Conveyance, therefore, is associated with an Owner. Owner type categories include a person, an organization, or a municipal or government agency. Each Owner can own one or more individual elements and can be part of a larger parent organization. For the example shown in figure 4, the well, treatment plant, and distribution system A might be owned by Town A, whereas distribution system B might be owned by Town B. Additional information stored for each Owner includes contact and address.

In summary, pairs of Sites are joined through unidirectional Conveyances, on which are recorded individual water Transfer/Volumes. Locations of Sites define the spatial representation of the water network, and information is stored about the Owner of each Site and Conveyance. These statements describe the basic conceptual core of the NJWaTr data model.

\section{Core Data Model}

The core NJWaTr E/R diagram (fig. 5) is an extension of the conceptual model described previously. (Only the definitions of the tables are shown in figure 5 because the details will be presented in later sections.) The core tables, along with their principal attributes and relationships, are described in the following paragraphs.

Each object that can be named or identified as the source or target of water movement in the wateruse network is called a Site. Sites can be as small as an individual well or as large as an aggregate of users in a County, if that is the only scale for which actual water-use data are available. Each Site is uniquely identified in the tblSite table by a Site_ID value and described by a Location and Owner (or owner organization). A Site that interacts directly with a water resource (withdrawals or returns) is paired with a Resource through an association table, tadSiteResource.

All water movements are described as exchanges between individual Site pairs. The connections between these Site pairs for water exchange are called Conveyances, and each Conveyance is uniquely identified in the table tblConveyance by a Conveyance_ID. Conveyances are modeled as strictly unidirectional; therefore, Sites that can exchange water in either direction will have two Conveyances, one for each direction. This is necessary to ensure that all water movements are recorded as non-negative values and to have complete control over defining Sites as either the source or target of individual water Transfers.

The Site-Conveyance relationship is strictly a 2:1 relationship (two Sites to each Conveyance), and it is the critical element in defining the water-use network. Two Sites form the ends of each Conveyance, one at the input (source) end and the other at the output (target) end. An association table, tadSite Conveyance, is used to pair the two Sites to a single Conveyance. Each Conveyance will have exactly two records in this table, one for each Site to which it joins. Only one From (source) Site and one To (target) Site can exist for each Conveyance_ID. The required non-PK attribute of tadSiteConveyance is the Site_ID 


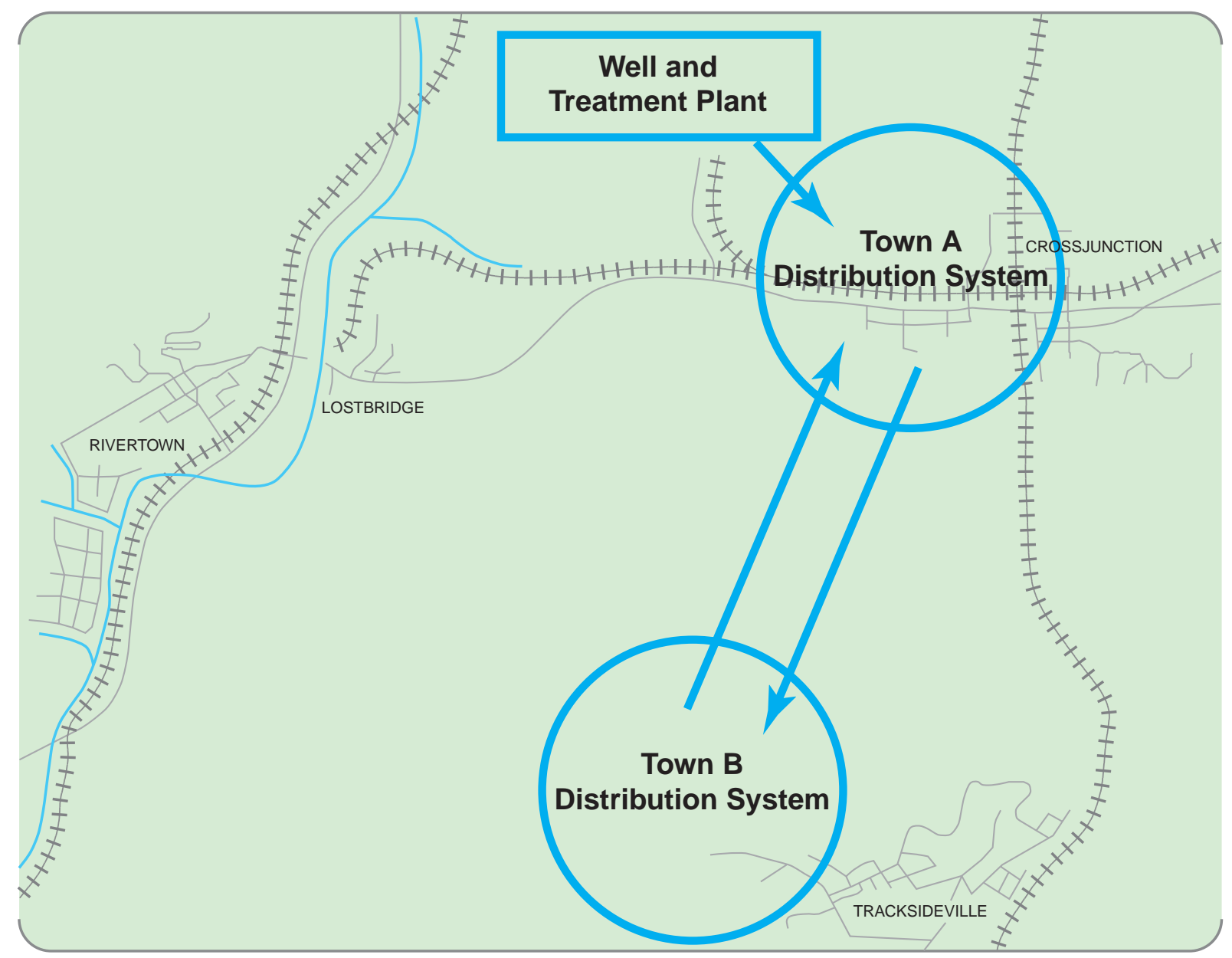

Figure 4. Spatial representation of sites and unidirectional conveyances of a small water network. 


\section{tblSite}

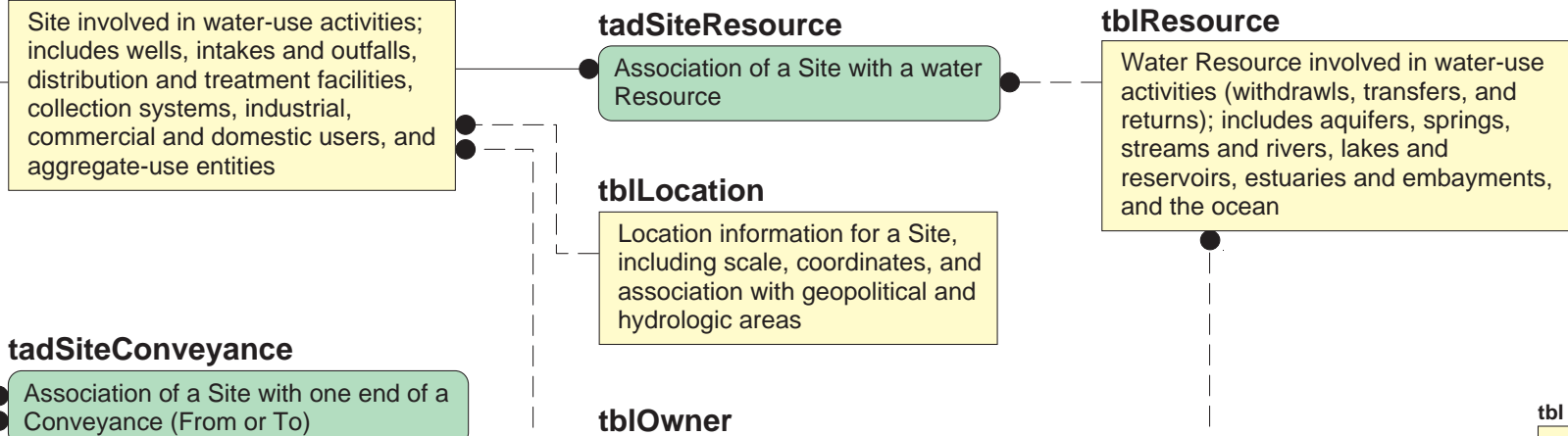

Owner organization or entity that owns, controls or monitors a Site, Conveyance - - - or water Resource, or is the parent organization for a Data Source

\section{tblConveyance}

Conveyance that serves as the

water-carrying connection between two

Sites (unidirectional); includes pipes,

canals and conduits, trucking, and

virtual linkages

\section{tasConveyanceOwner}

Association of a Conveyance with an

Owner

\section{tblTransfer}

Record of a water Transfer on a

Conveyance between two Sites for a specified Time Interval

\section{tblVolume}

Volume (quantity of water) recorded for

a Transfer based on a specific

\section{tdxDataSource}

Data being stored

\section{EXPLANATION}

Functional Table Types

tbl

tdx

tas

ASSOCIATION, SIMPLE; tas prefix; white

tad

ASSOCIATION, WITH DATA; tad prefix; green

Table and Relationship Symbols

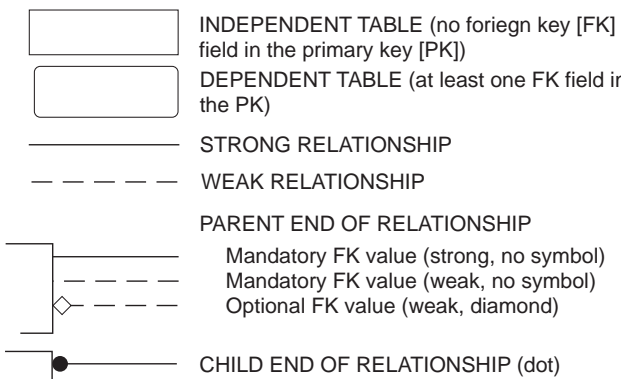

Figure 5. Core tables and relationships in NJWaTr. 
field, which identifies the Site at the From or To Conveyance end. The association table allows multiple Conveyances associated with individual Sites to be identified quickly, and the FromOrTo field assists in describing each Conveyance as an input or output connection to a Site. A hypothetical example of records in the tadSite Conveyance table is shown in figure 6, where the highlighted Conveyance identified as Conveyance_ID = " 28 " is determined by two queries to be a pipe connecting an intake on the Raritan River to an industrial facility. Many complex investigations of the data are possible through the association table tadSite Conveyance.

The Conveyance attributes include classifications of the type (pipe, aqueduct, and so forth) and the functional action they perform in the water network (such as "From intake pipe To single user" as shown in figure 6). Each Conveyance may be associated with one or more Owners through the tasConveyanceOwner table (fig. 5).

The Transfer entity stores the individual water-movement estimate or measurement made on each Conveyance during a single time interval. Each Transfer in the tblTransfer table is uniquely identified by a Transfer_ID and has the FK field Conveyance_ID to identify the Conveyance to which the Transfer applies. All Transfers are time-dependent, and a Transfer includes starting and ending dates and the time-interval classification of that date range (for example, year or month). Because different water-use values derived from different estimation methods must be stored separately, original reported volume values are stored in the Volume entity. The tblVolume table stores the reported/estimated Volume value with a unique Volume_ID, along with its original units, method of determination, and data source. One Volume for each Transfer is designated as the default, and its value is converted to million gallon equivalents and stored in the tblTransfer table for quick retrieval. The Volume entity, therefore, is not needed to retrieve volume estimates for routine summarization, but is available for additional details about the original Volumes and alternative estimates stored for each Transfer.

The last core entity is Owner. An Owner is a person, company, municipality, or other organization that controls and maintains Sites and Conveyances and can serve as the provider of data (DataSource) for Transfer Volumes. Each Owner is uniquely identified in the tblOwner table by an Owner_ID. Individual Owners can be part of an Owner organization, so a ParentOwner_ID value is identified as an attribute of an Owner record when applicable. This is especially useful for grouping Sites with single Owners or for exploring hierarchies of ownership.

The sections of this document that immediately follow describe in greater detail the structure of the subject areas of the NJWaTr model. The general use of Addresses, DataSources, Permits, Aliases, and UserDefined Details are discussed in separate sections.

\section{Site Subject Area}

The Site subject area focuses on the tblSite table and its related entities. The Site subject area diagram is shown in figure 7. Each Site is identified by a unique Site_ID. Each Site also is strictly classified as a particular SiteType and has a functional water-UseType assignment. For example, a well Site could be assigned a SiteType (discussed below) value of "withdrawal well" and a UseType value of "public supply." Sites are paired with Conveyance_IDs in the tadSiteConveyance table, which connects them (relationally speaking) in a direct path to Conveyance and Transfer information; data from any of these entities are available at the Site (or Site classification) level.

When an area aggregate, such as a town, is to be used as a Site, multiple Sites are entered into NJWaTr to represent different use components, or layers, for the area. For example, a town aggregate might 


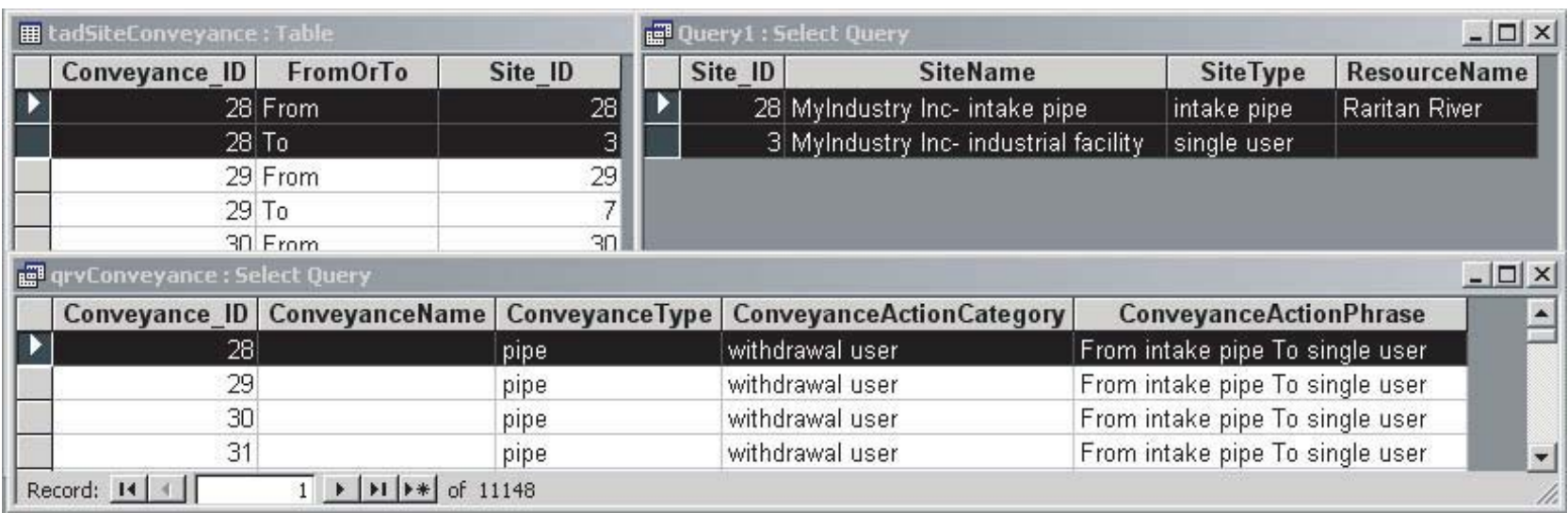

Figure 6. A hypothetical example of Conveyance data in NJWaTr showing records in table tadSite Conveyance highlighted for Conveyance_ID $=28$ and indicating From Site_ID $=28$ and To Site_ID = 3 (upper left), a query showing the identities of the Sites at the Conveyance ends From an intake pipe To an industrial facility (upper right), and a query showing the properties of the Conveyance (bottom). 
tbISite

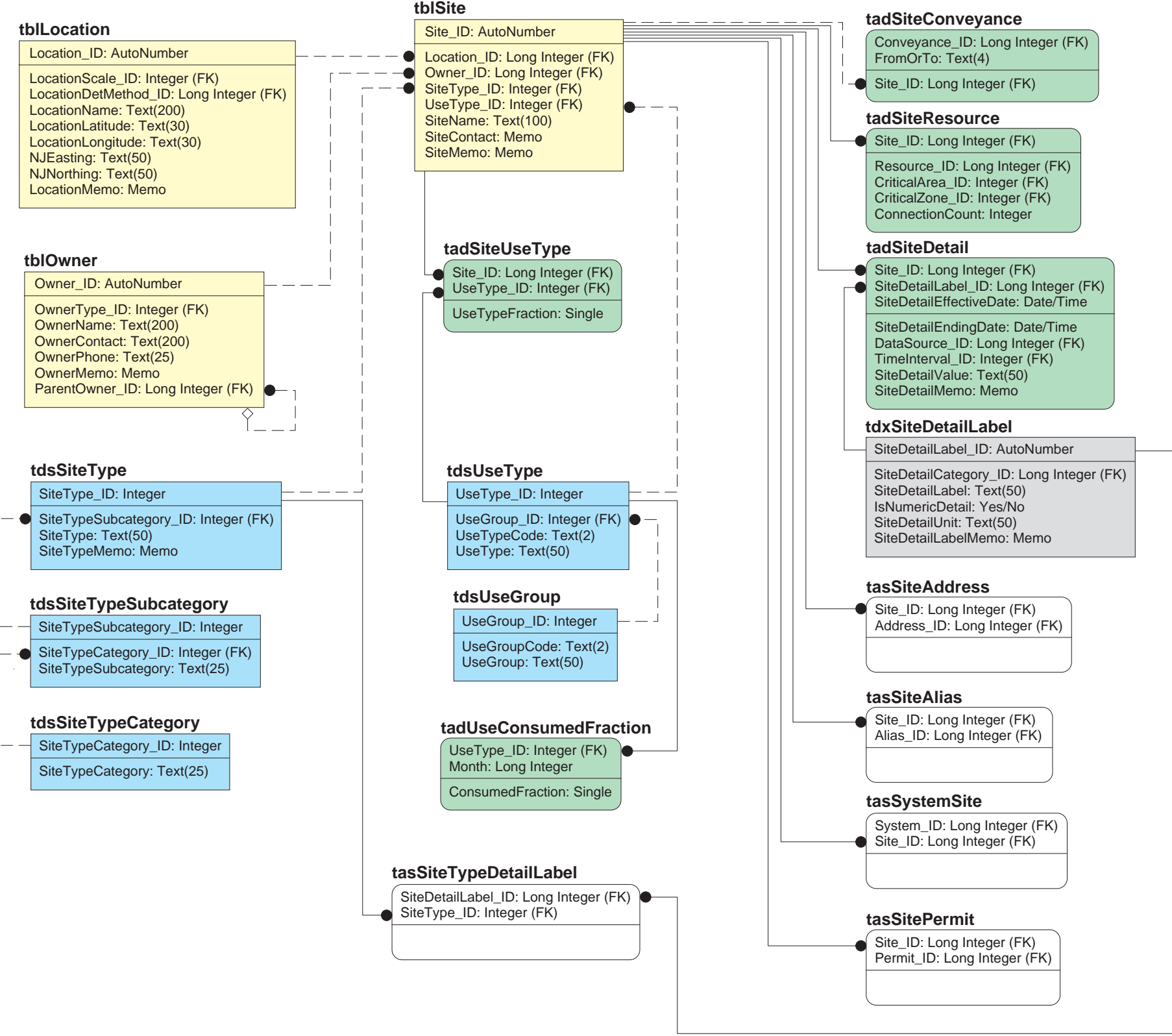

Figure 7. Site subject area tables, fields, and relationships. See Explanation following. 


\section{EXPLANATION}

Functional Table Types

tbl

BASIC DATA TABLE; tbl prefix; yellow

DOMAIN, STATIC; tds prefix; blue

tdx

DOMAIN, USER-EXTENDABLE; tdx prefix; gray

tas

ASSOCIATION, SIMPLE; tas prefix; white

tad

ASSOCIATION, WITH DATA; tad prefix; green
Table and Relationship Symbols

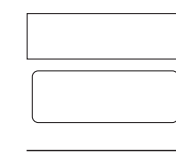

作 field in the primary key [PK])

(DEP ) the PK)

STRONG RELATIONSHIP

- - - - - WEAK RELATIONSHIP

PARENT END OF RELATIONSHIP

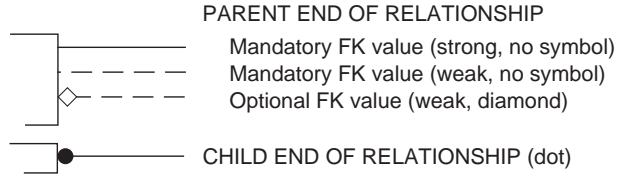

\section{Field Property Indicators}

PK PRIMARY KEY (PK) FIELDS ABOVE LINE PRE

(FK) FOREIGN KEY FIELD

(20) NUMBERS IN PARENTHESES MAXIMUM NUMBER OF CHARACTERS

Figure 7. Site subject area tables, fields, and relationships--Continued 
be represented by separate Sites for ground-water withdrawal, surface-water withdrawal, domestic users, irrigation, and commercial users. This partitioning of an area aggregate into separately identified and useclassified Site records allows detailed storage of water-use Transfers for the individual components of the aggregate, and it is consistent with storage of point-located Site data. By storing use components as separate Sites in all cases, available data from different scales can be entered, and data summarization is greatly enhanced.

Each Site record contains FKs that associate it with a single Owner and a single Location, both of which are attributes of the Site. Both entities have descriptive fields of their own that are available for use with the Site object for classification, sorting, and grouping Sites as needed. In particular, the Location represents 1 basic data, 4 association, and 9 domain tables, for a combined total of 48 non-key fields describing spatial, geopolitical, and watershed location information for a Site. The Location and Owner subject areas will be described farther on in separate sections. Both the Owner and Location can be shared by different Sites when appropriate. For example, Bottling Company X (Owner) has a plant facility (Location) with a well and an on-site treatment plant. The well, treatment plant, and facility itself (place of water use for bottling) would be entered and classified as separate Sites in NJWaTr, and all would share the same Location and Owner.

Each Site is given a name but may also be known by other names or through established codes used by different organizations. To accommodate multiple identification methods for single Sites, association tables are used to pair Sites with Permits and Aliases in tasSitePermit and tasSiteAlias, respectively. Likewise, address information stored for a Site is handled by an association with an Address record in tasSiteAddress. Storage of User-Defined Details about a Site is handled through the tadSiteDetail table. The Permit, Alias, Address, and User-Defined Details subject areas are described farther on in separate sections.

Each Site is described by a number of domain entities that classify and further identify the attributes or properties of a Site. These include the SiteType and UseType, and each domain applies a different standardized classification to an individual Site. The SiteType is a nested domain of tdsSiteTypeCategory, tdsSiteTypeSubcategory, and tdsSiteType that contain, for example, the values "treatment," "pre-use," and "potable-water treatment plant," respectively.

The type of water use represented by a Site is determined by a UseType. UseType is defined in NJWaTr using New Jersey classifications in the nested domains tdsUseGroup and tdsUseType which have, for example, the nested values "Agricultural" and "Cranberries." An optional table, tadUseConsumedFraction, is provided to store estimated monthly consumptive-use profiles for each UseType as a fraction of water lost to the atmosphere for a particular month at a Site. The data provide a means to create consumptive Transfers for Sites that have different UseTypes, to check available consumptive data for different Sites against predicted consumption by UseType, or to develop better monthly consumptive-use profiles for each type.

Another optional table, tadSiteUseType, is used to allow Sites with a designated primary UseType to be further identified as having secondary uses. The tadSiteUseType table pairs a Site_ID with a UseType_ID to form its PK and has an additional field, UseTypeFraction, to store the percentage of that UseType in the overall classification of the Site.

A third, optional Site-related table, tasSiteTypeDetailLabel, pairs SiteTypes with Label terms for a User-Defined Detail to establish valid combinations of types and labels. This table can be used to enforce or restrict the availability of certain labels to certain types in a data-entry application. 
Sites that interact directly with a water resource such as a river or reservoir are classified using the tdsSiteTypeCategory domain as "resource interactors." These Sites are associated with the Resource entity through the tadSiteResource association table, whose structure was discussed earlier in the description of tad functional tables. Each resource-interactor Site is paired with a single Resource, and the number of direct connections represented by the pairing (for example, the number of intake pipes on a reservoir) is stored in the field ConnectionCount. If a Site is a well in a regulated aquifer it may be assigned to a CriticalArea and CriticalZone; the domains $t d s$ CriticalArea and tdsCriticalZone will be discussed in the "Resource Subject Area" section.

Sites can be associated with other Sites through the tasSystemSite association table to form named Systems. A System, which allows any user-defined collection of Sites to be handled as a group, is discussed in more detail in the "System Subject Area" section.

Although each user implementation of NJWaTr will create its own Site entries, three generalized water-accounting Sites are provided for users in the tblSite table. The first Site has a SiteName of "Atmosphere (consumptive use)" and represents water that is evaporated or incorporated into products. This Site is used as the receiving (To) end of a Conveyance to capture Transfer Volumes that represent consumptive use for another Site; all Transfers on that Conveyance will represent consumptive use for the other Site. The second Site has a SiteName of "Unaccounted-for water" and represents the combination of leakage from distribution systems and public use of water, such as hydrant flushing, fire fighting, and street sweeping. This Site also is used as the receiving (To) end of a Conveyance to capture Transfer Volumes for those purposes from another Site (for example, to record unaccounted-for use for a regional or local distribution system Site). The third Site has a SiteName of "Inflow and Infiltration water" and represents the combination of inflow from surface water and infiltration from ground water into a wastewater-collection system. This Site is used as the sending (From) end of a Conveyance to capture Transfer Volumes as inputs to another Site. These three generalized Sites are used at either the From or To end of a Conveyance as appropriate and are convenient for identifying and gathering the Transfers that they represent (consumptive use, unaccountedfor use, and inflow/ infiltration).

\section{Conveyance Subject Area}

The Conveyance subject area focuses on the tblConveyance table and its related entities. The Conveyance subject area diagram is shown in figure 8 . Each Conveyance is identified by a unique Conveyance_ID. Sites are paired with Conveyance_IDs in the tadSiteConveyance association table, and Transfers inherit Conveyance_ID as an attribute. There is a direct path, therefore, connecting Sites to Transfers through the Conveyance; data from any of these entities are available at the Conveyance level in the database model.

A Conveyance initially is defined as a record in the tblConveyance table, but it needs established endpoints in the tadSiteConveyance association table as two paired Sites, one being identified as the source (From end) and the other as the target (To end) using the FromOrTo field. This results in two entries in the tadSiteConveyance table for each Conveyance. Conveyances are strictly unidirectional; therefore, two Conveyances are required to store data for bi-directional Transfers between two specific Sites (four records in the tadSiteConveyance table). Once established, a Conveyance serves as the parent to one or more water Transfers.

As described in the previous section, three special Sites are included in NJWaTr to handle consumptive water-use, unaccounted-for use, and inflow and infiltration water. The Conveyances created To the Sites named "Atmosphere (consumptive use)" and "Unaccounted-for water" will anchor water-network 


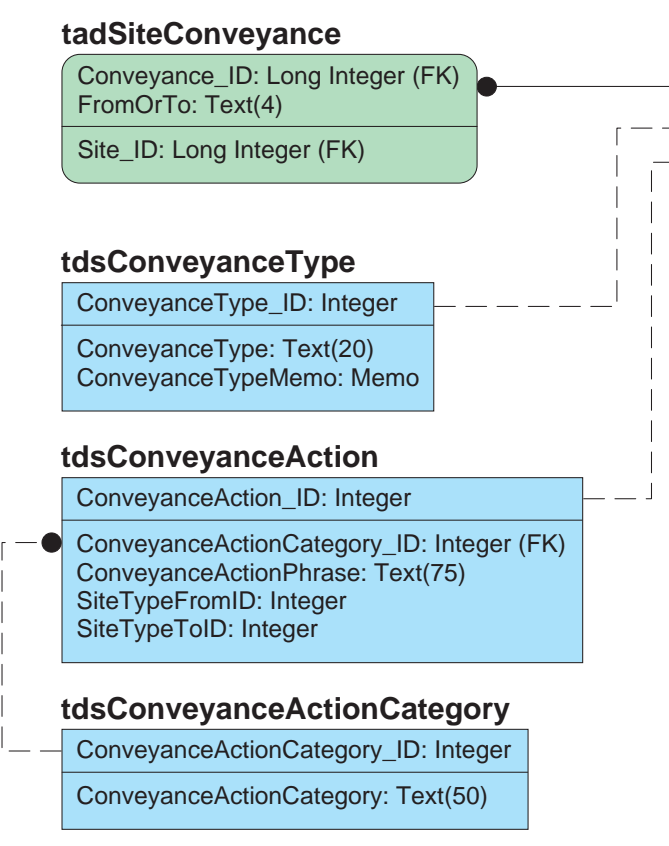

tblConveyance

Conveyance_ID: AutoNumber

ConveyanceType_ID: Integer (FK)

ConveyanceAction_ID: Integer (FK)

ConveyanceMemo: Memo

\section{tasConveyanceOwner}

Conveyance_ID: Long Integer (FK) Owner_ID: Long Integer (FK)

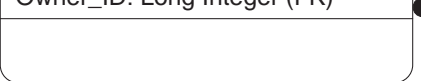

\section{tblOwner}

Owner_ID: AutoNumber

OwnerType_ID: Integer (FK)

OwnerName: Text(200)

OwnerContact: Text(200)

OwnerPhone: Text(25)

OwnerMemo: Memo

ParentOwner_ID: Long Integer (FK)

\section{tblTransfer}

Transfer_ID: AutoNumber

Conveyance_ID: Long Integer (FK) Timelnterval_ID: Integer (FK) TransferEffecliveDate. Date/Time TransferEndingDate. Date/Time

Transfer: Double

TransferMemo: Memo

\section{tasConveyanceAlias}

Conveyance ID: Long Integer (FK)

Alias ID: Long Integer (FK)

\section{tadConveyanceDetail}

Conveyance_ID: Long Integer (FK)

ConveyanceDetailLabel ID: Long Integer (FK)

ConveyanceDetail: Text(50)

ConveyanceDetailMemo: Memo

\section{tdxConveyanceDetailLabel}

ConveyanceDetailLabel_ID: AutoNumber

ConveyanceDetailLabel: Text(50)

ConveyanceDetailLabelMemo: Memo

\section{tadConveyanceExpectedFraction}

\section{Conveyance ID: Long Integer (FK)}

ExpectedFractionEffectiveDate: Date/Time

ExpectedFractionEndingDate: Date/Time

ExpectedFraction: Single

\section{EXPLANATION}

Functional Table Types

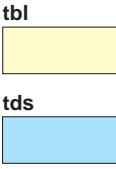

BASIC DATA TABLE; tbl prefix; yellow

DOMAIN, STATIC; tds prefix; blue

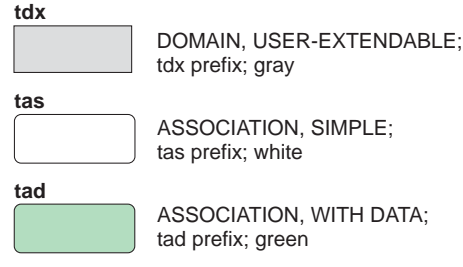

Table and Relationship Symbols

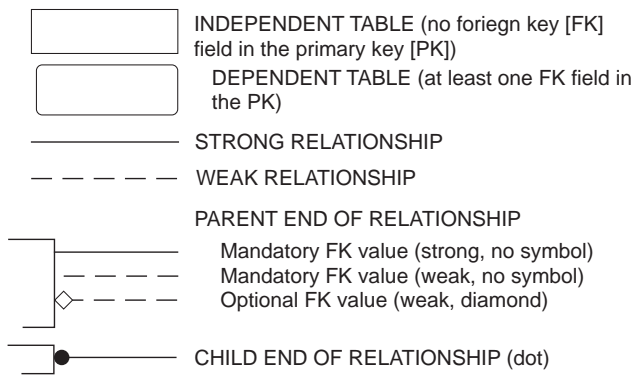

\section{Field Property Indicators}

PK PRIMARY KEY (PK) FIELDS ABOVE LINE NON-PK FIELDS BELOW LINE

(FK) FOREIGN KEY FIELD

(20) NUMBERS IN PARENTHESES INDICATE TEXT FIELD CHARACTERS

Figure 8. Conveyance subject area tables, fields, and relationships. 
Volume losses for the Sites at the From ends of those Conveyances, whereas Conveyances created From the "Inflow and Infiltration water" Site will handle those undefined inputs to a Site at the To end of the Conveyance. All consumptive use is, therefore, easily recognized by its Conveyance to the Atmosphere Site, and the tadSiteConveyance table can be used to quickly retrieve all Sites involved in consumptive use. The retrieval of unaccounted-for water losses and inflow/infiltration water inputs are handled in the same way.

A Conveyance may have one or more Owners responsible for control and maintenance, and this is handled by pairing Conveyances with Owners in the association table tasConveyanceOwner. Multiple ownership assignment is useful, for example, when a Conveyance represents water exchange between two towns and exploring the water network connections to either town (the Owner) would reveal the Conveyance.

Each Conveyance has an optional name (ConveyanceName) and is provided access to the tblAlias table as needed through the tasConveyanceAlias association table. Storage of User-Defined Details about a Conveyance is handled through the tadConveyanceDetail table. The Alias and User-Defined Details subject areas are described elsewhere.

Two domain tables are used to classify each Conveyance: tdsConveyanceType and tdsConveyanceAction. The tdsConveyanceType domain includes the values "pipe," "canal," "virtual," and others. Virtual Conveyances are connections between Sites, such as a connection between a town and a treatment plant, but without detailed data about the Conveyance itself. In this example, the actual number of pipes may not be important or even known but identifying the connection is critical to capturing Transfer information between the town and treatment plant.

The tdsConveyanceAction domain contains a ConveyanceActionPhrase field that describes the function of the Conveyance based on the SiteTypes at the source and target ends, for example "From withdrawal well To potable-water treatment plant." The nested domains $t$ sConveyanceActionCategory and tdsConveyanceAction can be used to identify Conveyances that serve a particular function within the wateruse network or to contrast Transfer/Volume data between action types, such as comparing ground-water withdrawals sent to treatment plants with ground-water withdrawals sent directly to distribution systems.

Not all SiteTypes can be paired through a connection (for example, a withdrawal well should not lead to a river intake pipe), so the ConveyanceAction domain is static and contains only valid combinations created from the SiteType domain list. The purpose of the static ConveyanceAction domain, therefore, is to limit the possible types of Site connections. Key values of the associated SiteTypes for a particular ConveyanceAction are stored as the attributes SiteTypeFromID and SiteTypeToID. These pseudo-key fields are not connected directly to the tdsSiteType table to avoid a circular relationship in the model (two separate pathways to join tables), but they can be linked through a query whenever needed to extend the ConveyanceAction domain manually to the SiteType domain. The ConveyanceActionPhrase field contains values created through a maintenance query (described in the "Conveyance Action Phrase Updates" section) to ensure a semantically accurate phrase defined by the SiteType of the Sites.

\section{Transfer/Volume Subject Area}

The Transfer/Volume subject area focuses on the tblTransfer and tblVolume tables and their related entities. The Transfer/Volume subject area diagram is shown in figure 9. Water Transfers between Sites through Conveyances represent the most detailed data in NJWaTr, and storing the full history of Transfers over time will result in the tblTransfer and tblVolume tables containing more records than other tables in the NJWaTr database. Each Transfer is identified by a unique Transfer_ID, and Transfers inherit the 


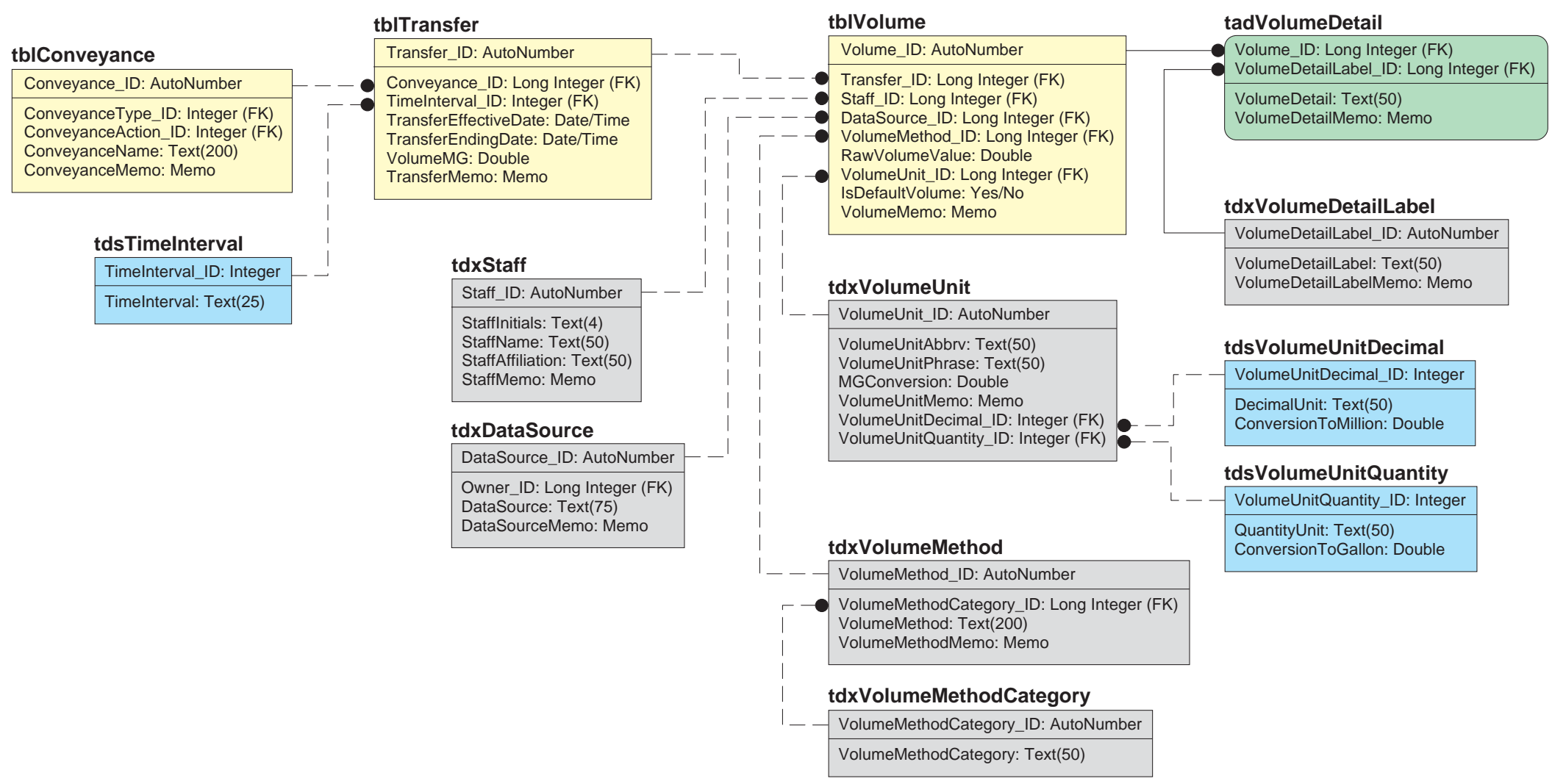

\section{EXPLANATION}

Functional Table Types

tbl

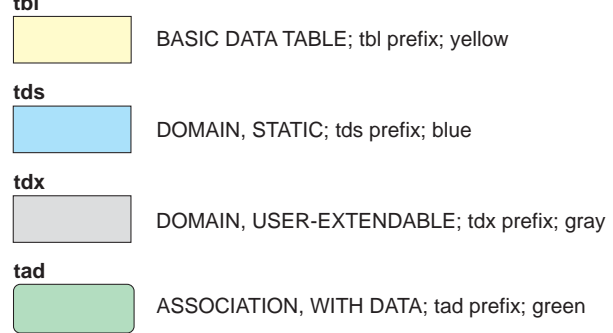

Table and Relationship Symbols

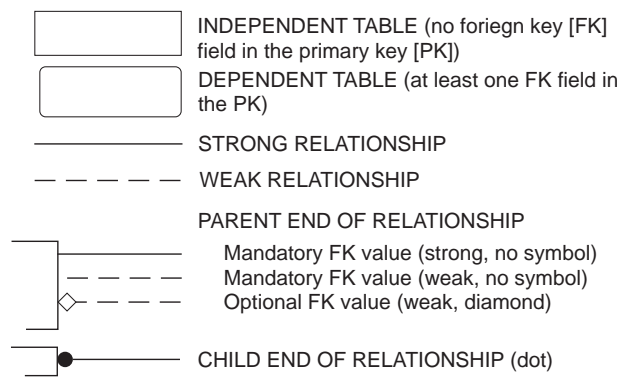

\section{Field Property Indicators}

PK PRIMARY KEY (PK) FIELDS ABOVE LINE NON-PK FIELDS BELOW LINE

(FK) FOREIGN KEY FIELD

(20) NUMBERS IN PARENTHESES INDICATE TEXT FIELD CHARACTERS

Figure 9. Transfer/Volume subject area tables, fields, and relationships. 
Conveyance_ID of their parent Conveyance as an attribute of each Transfer. There is, therefore, a direct relational pathway to Site and Conveyance information from the Transfer level of the database model. Details of each Transfer in original reporting units are stored in the table tblVolume.

Transfers are time dependent. Each Transfer is bounded, inclusively, by its two date fields, TransferEffectiveDate and TransferEndingDate. The interval category that the date range defines is classified by a value from the tdsTimeInterval domain table ("year," "month," and so forth). It is assumed that a single-day datum represents the smallest recording interval for Transfer/Volume data. The TimeInterval domain is useful for aggregating data from different time scales and for analyzing the occurrence frequency of different scales for various Conveyance and Site types. Other time scales-fiscal years (Federal and state), water years, and seasons — can be developed through queries on the bounding date fields and are not designed into the database.

NJWaTr specifications require that Volumes for a specific Transfer be allowed to vary on the basis of different determination methods. This facilitates study of differences in the determination methods used for the summarization and reporting of water-use activities. Original Volume values (as provided by the DataSource), therefore, are stored along with their descriptive attributes in the table tblVolume. The relationship between tblTransfer and tblVolume is modeled as one-to-many with many Volume estimate alternatives possible for each Transfer. When there is more than one Volume record, a field in tblVolume, IsDefaultVolume, identifies the particular Volume record to be associated by default with the Transfer; this Volume is converted to common units and stored in the VolumeMG field in the tblTransfer table using a maintenance query described in the section "Volume Conversion to Common Units and Updating Transfers." When only a single Volume is stored for a Transfer, it is always the default Volume. Having a single default Volume stored in converted form in the tblTransfer table also means that, for most data exploration and reporting activities, the tblVolume table and its associated domains are not needed.

The original data for each Transfer are stored in the tblVolume table. The RawVolumeValue field stores the numeric part of a reported Volume value. For example, "1.45" would be stored for 1.45 thousand cubic feet. It is important to store all Volumes in their reported form to facilitate checking against original DataSources. Within the tblVolume table, the RawVolumeValue is associated with its original VolumeUnit, its method of determination (nested domain of VolumeMethod and parent Category), a DataSource, and the name/affiliation of the Staff responsible for the entry. The $t d x$ VolumeUnit domain table represents a domain cluster and allows a VolumeUnit to be created from any combination of decimal and quantity dimensions used in the original reporting units (for example, Volumes reported in thousand cubic feet have separate unit component terms of "thousand" and "cubic-feet"). Each VolumeUnit also is provided with a value for the MGConversion field, which is the value by which the RawVolumeValue is multiplied to convert it to common units regardless of the reporting units. The RawVolumeValue is converted on the basis of the VolumeUnit to an equivalent value in million gallons (MG) units, and that converted Volume value is stored in the tblTransfer table in the field VolumeMG.

During initial data entry or batch loading, VolumeMG in tblTransfer is assigned a default value of "-1" for new Transfer records, and a maintenance query (described in the "Volume Conversion to Common Units and Updating Transfers" section) is run to apply the VolumeUnit MGConversion against the RawVolumeValue in order to update the Transfer VolumeMG value. The conversion factors are developed for any VolumeUnit combination by automatically creating the product of each component conversion (decimal and quantity). When new VolumeUnits are added to the domain, a maintenance query (described in the "Volume Unit Updates" section) is used to update the VolumeUnitPhrase and MGConversion fields in $t d x$ VolumeUnit on the basis of the selection of a unique combination of components. The tdxVolumeUnit table also could be used in reverse, through queries, to convert the commonly used million gallons, VolumeMG values, to any other units desired. 
One additional feature of the Transfer/Volume subject area, the $t d x$ VolumeDetail table, is shown in figure 9. Additional data storage is provided for variables that are not known in advance or for variables that are associated with some Volumes but not others. This is accomplished through the use of a User-Defined Detail table. (User-Defined Detail tables are discussed in the "User-Defined Details" section.) The association of a Volume_ID with a VolumeDetail Label_ID and a VolumeDetail in the tadVolumeDetail table can accommodate any optional attributes for Volumes, for example, "Accuracy." Accuracy values can be associated with each Volume by assigning a value of "Accuracy" to a record in the VolumeDetailLabel field of the $t d x$ VolumeDetailLabel domain and recording the accuracy estimate in the VolumeDetail field for each Volume_ID. In addition, a note about the definition of accuracy for the NJWaTr implementation can be entered in the VolumeDetailLabelMemo field.

\section{Location Subject Area}

The Location subject area focuses on the tblLocation table and its related entities. The Location subject area diagram is shown in figure 10. Location is modeled as an attribute of a Site. The Location_ID as a FK field in the tblSite table provides the full collection of Location attributes and related domains to individual Sites. Through the Site's relationships, Location fields can be used as attributes that also provide spatial characterization of Conveyances and Transfers. A single Location may accurately serve more than one Site if they occur in the same place, such as individual Site-level objects that represent various wateruse types (for example, livestock or irrigation) of an aggregate Site. Site aggregates were discussed previously in the "Site Subject Area" section.

Each Location is identified by a unique Location_ID and characterized by two domains that represent LocationScale (point, or various area categories) and the method used to determine a Location (LocationDetMethod). Optional fields are provided for a LocationName, the mapping coordinates LocationLatitude and LocationLongitude, and the New Jersey grid coordinates NJEasting and NJNorthing. Mapping coordinates can store values that represent a point location or the centroid of a larger area.

A Location is associated with a geopolitical state, county, and MCD nested domain hierarchy. Although nested, the geopolitical domains are each associated separately with tblLocation using the association tables tadLocationState, tadLocationCounty, and tadLocationMCD. Each association table is used to pair a Location with one or more of the geopolitical selections, along with the proportion of the Location represented by the individual pairings using a Fraction field. This allows an area Location that overlays parts of two towns, for example, to indicate the fraction of the Location represented by each town. This information can be used to apportion water-use activities for an area Site Location among the individual geopolitical areas. When more than one item from the domain is associated with a single Location, one of the associates should be designated as the primary selection to be retrieved by default with the Location by having a "Yes" value in the IsPrimary field in the association table; all other associates would be given a "No" value. When only a single item from a domain is associated with a Location, it is designated the primary selection.

The State of New Jersey is divided into six Drought Regions that are listed in the domain table tdsDroughtRegion. Because single towns (MCD-scale) belong entirely to a single Drought Region, the $t d s M C D$ domain includes a FK field that identifies the Drought Region to which it belongs. Drought Region classification, therefore, also is available to counties and states and to all water-transfer activities through the Location's Site(s) and related entities.

Locations also can be associated with one or more standard hydrologic units through the association table tadLocationHUC. The $t d s H U C$ domain contains a list of the 933 New Jersey 14-digit watersheds, 
tdsLocationScale

LocationScale_ID: Integer

LocationScale: Text(50)

tdxLocationDetMethod

LocationDetMethod_ID: AutoNumber

LocationDetMethod: Text(50)
tblLocation

Location_ID: AutoNumber

LocationScale ID: Integer (FK)

LocationDetMethod ID: Long Integer (FK)

LocationName: Text(200)

LocationLongitude: Text(30)

NJEasting: Text(50)

LocationMemo: Memo
tblSite

Site ID: AutoNumber

Location ID: Long Integer (FK) Owner_ID: Long Integer (FK) SiteType_ID: Integer (FK)

SiteName: Text(100)

SiteContact: Memo

SiteMemo: Memo

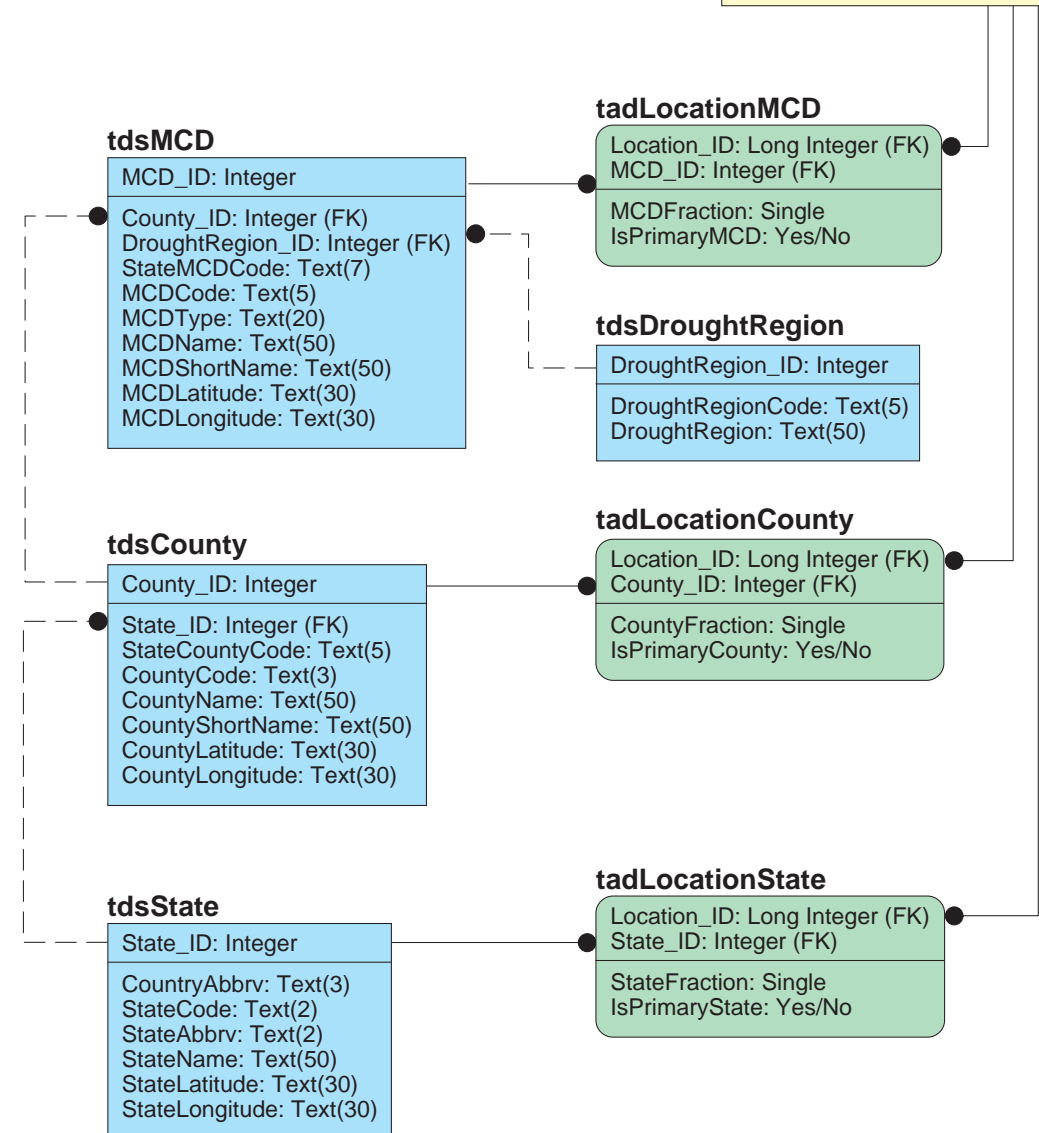

tadLocationHUC

Location_ID: Long Integer (FK)

HUC ID: Integer (FK)

HUCFraction: Single

IsPrimaryHUC: Yes/N

\section{tdsHUC}

HUC_ID: Integer

WatershedCode: Text(20)

WaterMgmntArea ID: Integer (FK)

HUC14: $\operatorname{Text}(14)$

HUC14Name: Text(50)

HUC11: Text(11)

HUC11 Name: $\operatorname{Text}(50)$

HUC8Name: Text(50)

WaterMgmntArea_ID: Integer

WaterRegion ID: Integer (FK)

WaterMgmntArea: Text(50)

\section{tdsWaterRegion}

WaterRegion_ID: Integer

WaterRegionCode: Text(50)

WaterRegion: Text(50)

\section{EXPLANATION}

Functional Table Types

(

$\operatorname{tdx}$

tad

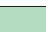

ASSOCIATION, WITH DATA; tad prefix; green

Table and Relationship Symbols

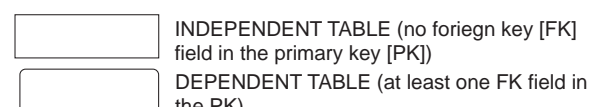

STRONG RELATIONSHIP PARENT END OF RELATIONSHIP

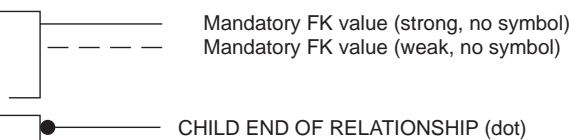

Field Property Indicators

PK PRIMARY KEY (PK) FIELDS ABOVE LINE NON-PK FIELDS BELOW LINE

(FK) FOREIGN KEY FIELD

(20) NUMBERS IN PARENTHESES INDICATE TEXT FIELD CHARACTERS

Figure 10. Location subject area tables, fields, and relationships. 
along with their higher 11- and 8-digit codes and basin names. This could have been modeled as a nested hierarchy, but the use of the denormalized form of this static domain is convenient when each Location is required to be associated with a 14-digit HUC. Because a single area Location may cross basin boundaries, more than one HUC may be associated with it, and the association table allows storage of the fraction of the Location in each HUC. One HUC may be designated as the primary selection from the domain to be used by default.

The State of New Jersey is divided into five Water Regions, and each of those is further subdivided into Watershed Management Areas (Hoffman and Lieberman, 2000). Watershed Management Areas are represented in NJWaTr by the nested domains tdsWaterRegion and tdsWaterMgmntArea. Each 14-digit HUC is associated with only one Water Management Area, so the $t d s H U C$ domain contains a FK field that points to the tdsWaterMgmntArea domain. In this way, each 14-digit HUC is classified by Watershed Management Area and its parent Water Region.

Each spatial domain table has a single record coded as "none identified." This allows a Site Location to be defined at a large scale (for example, county-scale data are not applicable to individual MCDs) or as an irregular area that does not correspond to geopolitical boundaries, such as a regional distribution system. For larger-than-MCD area Locations, the tadLocationMCD table would pair the Location with the MCD record that represents "unidentified;" for larger-than-County Locations, both the county and MCD association tables would classify the Location as unidentified for those scales.

\section{Owner Subject Area}

The Owner subject area focuses on the tblOwner table and its related entities. The Owner subject area diagram is shown in figure 11. An Owner is a person, company, municipality, or other organization that controls and maintains a Site, Conveyance, or Resource, or that can serve as the provider of a DataSource for Transfer Volumes, Permit assignments, and User-Defined Details in the database. Each Owner is identified by an Owner_ID value; aggregate areas represented as single Sites, such as a town, may have no Owner in this context and would be assigned an Owner_ID corresponding to a value of "No Owner." Different kinds of Owners are classified through the tdsOwnerType domain (for example, "Private," "Municipal," or "State"). Owners are further described by fields for OwnerName, OwnerContact, and OwnerPhone, and an Owner's Address is handled by an association through the tasOwnerAddress table with the tblAddress table (to be discussed in the "Address Subject Area" section).

Sites, Resources, and DataSources are associated with a single Owner by inheriting the Owner_ID as a FK attribute. The absence of a valid Owner assignment is indicated by using the Owner_ID = "0" record coding for "No Owner." Conveyances can have one or more Owners (for example, recognizing joint ownership of a connection between towns), which is accommodated through the tasConveyanceOwner association table. Owners also can be associated with one or more Permits, which is accommodated through the tasOwnerPermit table.

Individual Owners can be identified as part of an Owner organization through the recursive hierarchical association of a ParentOwner_ID with a different Owner record (a self-join). Nesting of Owners provides a way to group Sites and Conveyances into actual organizational units and to explore hierarchies of ownership. 
tblOwner

\section{tbISite}

Site_ID: AutoNumber

Location_ID: Long Integer (FK)

Owner_ID: Long Integer (FK)

SiteType_ID: Integer (FK)

UseType ID: Integer (FK)

SiteName: Text(100)

SiteContact: Memo

SiteMemo: Memo

\section{tblResource}

\section{Resource_ID: AutoNumber}

WaterBodyType_ID: Integer (FK)

Owner_ID: Long Integer (FK)

ResourceName. Text(200)

ResourceCodeName: Text(200)

ResourceMemo: Memo

\section{tasConveyanceOwner}

Conveyance_ID: Long Integer (FK)

Owner_ID: Long Integer (FK)

\section{tblConveyance}

Conveyance_ID: AutoNumber

ConveyanceType_ID: Integer (FK)

ConveyanceAction_ID: Integer (FK)

ConveyanceName: Text(200)

ConveyanceMemo: Memo

\section{Functional Table Types}

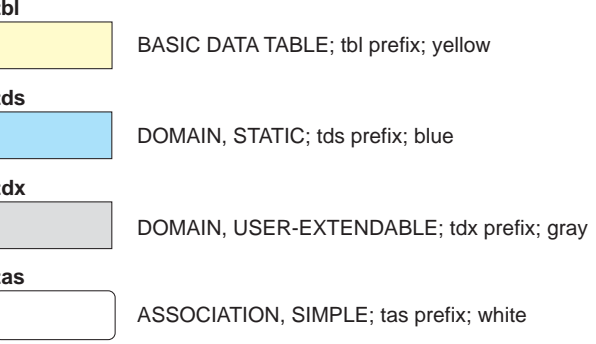

OwnerType ID: Integer (FK)

Owneriding

OwnerName: Text(200)

OwnerPhone: Text(25)

OwnerMemo: Memo

ParentOwner_ID: Long Integer (FK)

\section{tdsOwnerType}

OwnerType_ID: Integer
OwnerType: Text(25)
OwnerTypeMemo: Memo

OwnerTypeMemo: Memo

\section{EXPLANATION}

\section{Table and Relationship Symbols}

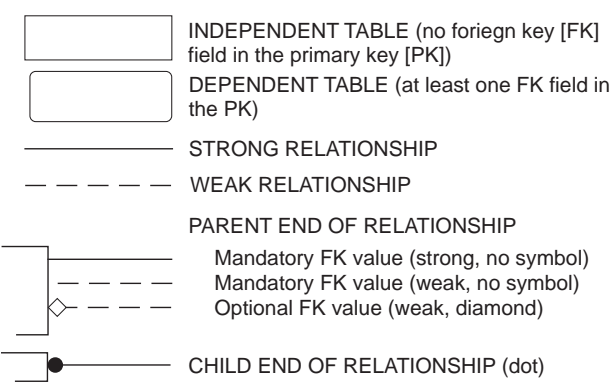

tdxDataSource

DataSource_ID: AutoNumber

Owner_ID: Long Integer (FK)

DataSourceMemo: Memo

\section{asOwnerAddress}

Owner_ID: Long Integer (FK) Address_ID: Long Integer (FK)

\section{tasOwnerPermit}

Owner ID: Long Integer (FK)

Permit_ID: Long Integer (FK)
DataSource: Text(75)

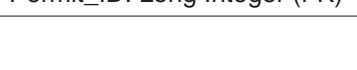

Figure 11. Owner subject area tables, fields, and relationships. 


\section{Resource Subject Area}

The Resource subject area focuses on the tblResource table and related entities. The Resource subject area diagram is shown in figure 12 . The NJWaTr model provides the tblResource table to store identification information for all aquifers, rivers, streams, reservoirs, lakes, ponds, and estuaries associated with wateruse activities.

Resources should not be equated with Locations in NJWaTr. Locations serve to identify the places where Sites reside, whereas Resources identify water bodies in the major categories of aquifer, river/stream, and reservoir, among others. Resources are independent of Sites although those Sites that interact with a water Resource will likely have a Location that matches the position of some part of its associated Resource. To meet potential future needs, the Resource subject area entities and attributes can extend NJWaTr by linking to hydrologic and geopolitical areas, gage information, ecology, and other water-body specific data.

A Site that interacts with a Resource is classified as a "resource interactor" (SiteTypeCategory) and is paired with a single Resource in the tadSiteResource association table that was discussed previously in the description of tad tables. The table pairs a Site with a single Resource and adds an optional field, ConnectionCount, to record the number of connections represented by the pairing (individual pipes or wells). Two domains also are used to classify the individual Site-Resource combination, tdsCriticalArea and $t d s$ CriticalZone. These classifications concern diversion sources or withdrawals in either of two regulated aquifer areas defined within New Jersey (Hoffman and Lieberman, 2000). These are not fully nested domains so they are applied individually to the Site-Resource pairings. Sites that are resource interactors located outside the critical areas receive an assignment of "not applicable" from the tdsCriticalArea domain. Sites receive the appropriate $t d s$ CriticalZone assignment on the basis of the type and location of withdrawal. From the relationships through the tadSiteResource table, wells associated with defined critical areas are recognized, and all withdrawals and releases for any water body listed in the tblResource table are available.

Each Resource is identified by a Resource_ID, a mandatory ResourceName, and an optional ResourceCodeName. Two name fields are used because names of water bodies are typically redundant even within the same watershed. The stream name of Mill Creek in New Jersey is a good example. The ResourceName field is intended for storage of the name in common use (for example, "Mill Creek"), whereas the ResourceCodeName field is used for an extended version of the name that provides unique identification information. A standard naming rule for ResourceCodeName has not been specified in NJWaTr, but the suggested practice is to distinguish different water bodies with the same ResourceName in the database by including the name of the town nearest its source in the ResourceCodeName. Thus, the ResourceCodeNames of "Mill Creek near Wildwood Crest, NJ" and "Mill Creek near Ocean City, NJ" are distinguishable although both have the same common name (ResourceName), "Mill Creek." That distinction facilitates searches in the database for Sites that interact with only one of those water Resources. Development and implementation of the naming scheme does not affect the current structure of NJWaTr in any way because only the contents of the ResourceCodeName field are affected.

Each Resource receives a FK to identify it with an Owner from the tblOwner table. Where there is no actual Owner (such as for any water Resource held in trust by the State of New Jersey), a "Not Owned" selection from the tblOwner table is used. In addition, the nested ResourceType and WaterBodyType domains provide a classification system for individual Resources. The tdsResourceType domain table identifies ground- or surface-water sources and a salinity attribute (freshwater, brackish, or saline) and applies a ResourceType name to each combination (such as "surface-water, fresh"). These basic classifications then are assigned as parent categories for individual selections in the tdsWaterBodyType table, which includes values such as "Reservoir," "River," and "Estuary." These domains and their relationships in NJWaTr allow 


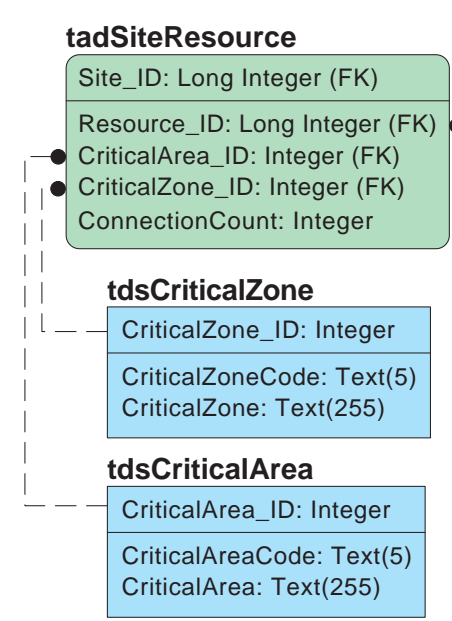

tblResource

\begin{tabular}{|l|l|} 
& Resource_ID: AutoNumber \\
WaterBodyType_ID: Integer (FK) \\
Owner_ID: Long Integer (FK) \\
ResourceName: Text(200) \\
ResourceCodeName: Text(200)
\end{tabular}

tasResourceAlias

Resource_ID: Long Integer (FK)

Alias_ID: Long Integer (FK)

tadResourceStructure

- ParentResource_ID: Long Integer (FK)

ChildResource_ID: Long Integer (FK)

ParentResourceFraction: Single

IsPrimaryResource: Yes/No

\section{adResourceDetail}

Resource_ID: Long Integer (FK)

ResourceDetailLabel_ID: Long Integer (FK) ResourceDetailEffectiveDate: Date/Time

ResourceDetailEndingDate: Date/Time

DataSource_ID: Long Integer (FK)

ResourceDetail: Text(50)

ResourceDetailMemo: Memo

\section{tdxDataSource}

DataSource_ID: AutoNumber $\ldots-\ldots-\ldots$

Owner_ID: Long Integer (FK)

DataSource: Text(75)

DataSourceMemo: Memo

tdxResourceDetailLabel

ResourceDetailLabel_ID: AutoNumber

ResourceDetailLabel: Text(50)

ResourceDetailLabelMemo: Memo
Functional Table Types

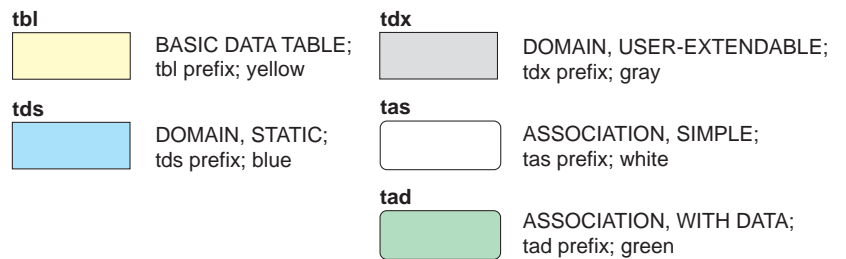

tad prefix; green

\section{EXPLANATION}

Table and Relationship Symbols

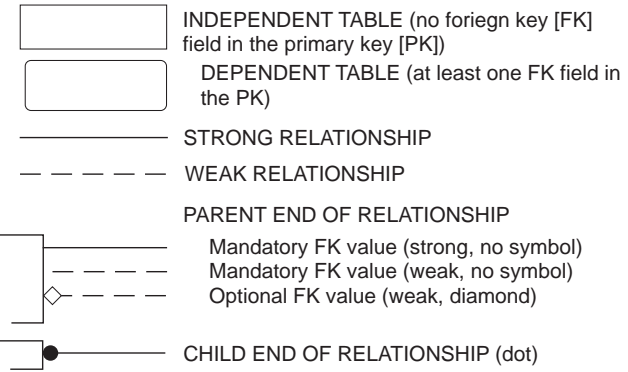

Field Property Indicators

PK PRIMARY KEY (PK) FIELDS ABOVE LINE NON-PK FIELDS BELOW LINE

(FK) FOREIGN KEY FIELD

(20) NUMBERS IN PARENTHESES INDICATE TEXT FIELD MAXIMUM NUMBER OF

Figure 12. Resource subject area tables, fields, and relationships. 
the identification of those Sites (and, therefore, their Conveyances and Transfers) involved with different types of water Resources.

Sometimes a single Site's interaction with water Resources cannot be limited to a single Resource, for example when a deep well has multiple openings in different aquifers. The tadSiteResource table does not allow multiple Resources per Site, so another method is provided to store and decode a composite Resource for a Site. The composite (parent) Resource is entered as a single record in tblResource and given an appropriate, informative ResourceName; the subcomponent (child) Resources of the composite also are entered individually. The table tadResourceStructure, discussed previously in the tad functional type description, pairs the Resource_ID of the composite Resource (as ParentResource_ID) with the Resource_ID of each subcomponent Resource (as ChildResource_ID), along with an optional fraction of the composite parent represented by the child. The structure table allows composite Resources to be recognized by their components, components to be recognized as occasionally associated with others, and all Sites associated with a particular Resource to be revealed even when the specified Resource is only one part of a larger composite.

Resources can have Aliases stored in the tblAlias table through the tasResourceAlias association table. The ability to store optional information about any particular Resource is provided by the tadResourceDetail table and its associated tdxResourceDetailLabel and tdxDataSource tables. This allows dams to be associated with reservoirs, fisheries to be associated with particular rivers, and any other optional attributes to be classified and stored as needed. Both the Alias and User-Defined Details subject areas are described elsewhere in this report. A related, optional table, tasWaterBodyDetailLabel, pairs WaterBodyTypes with Label terms for a User-Defined Detail to establish valid combinations of types and labels. This table can be used to enforce or restrict the availability of certain labels to certain types in a data-entry application.

\section{System Subject Area}

The core NJWaTr data structures that join Sites through unidirectional Conveyances describe a water network by allowing each Site to show the characteristics of its input and output connections and related Transfers. Sites can be grouped by their descriptive attributes and Type domains, and by the attributes of their Conveyances, Transfers/Volumes, Locations, Owners, and associated Resources. For convenience, an additional grouping of Sites called Systems can be set up in NJWaTr to gather Sites for any purpose, regardless of whether or not they are connected or share any attributes. For example, a public-supply System could be created to include all Sites that represent withdrawals (wells, intakes), treatment, and distribution. Other Systems could include the cluster of Sites that make up a fish hatchery operation, an industrial complex, the distribution network of Sites for a town, a regional-collection system, or the collection of Sites handled as a discrete unit during data entry.

Three tables-tdxSystem, tdxSystemType, and tasSystemSite - are required to set up System-level identities for Sites in NJWaTr, and these tables and their relationships are illustrated in figure 13. Systemidentity information is stored in the table $t d x$ System. Each System is given a unique System_ID and a SystemName. A SystemType classification is assigned from the $t d x$ SystemType domain and can include such values as "Public Supplier" and "Public Wastewater System." An association table, tasSystemSite, is used to pair individual Sites (from tblSite) with individual Systems. A System can consist of any number of Sites, and each Site can be a member of any number of Systems. This structure allows the NJWaTr user to select all Sites associated with a System or to identify all Systems associated with a Site and to generate membership statistics, such as the number and type of Sites that belong to three or more Systems. 


\section{tbISite}

\begin{tabular}{|l|l|}
\hline Site_ID: AutoNumber & tasSystemSite \\
\hline Location_ID: Long Integer (FK) \\
Owner_ID: Long Integer (FK) \\
SiteType_ID: Integer (FK) \\
UseType_ID: Integer (FK) \\
SiteName: Text(100) \\
SiteContact: Memo \\
SiteMemo: Memo \\
\hline
\end{tabular}

\section{tdxSystem}

System ID: AutoNumber

SystemType_ID: Long Integer (FK)

SystemName: Text(200)

SystemMemo: Memo

ParentSystem_ID: Long Integer (FK)

\section{tdxSystemType}

SystemType_ID: AutoNumber

SystemType: Text(40)

SystemTypeMemo: Memo

\section{EXPLANATION}

Functional Table Types

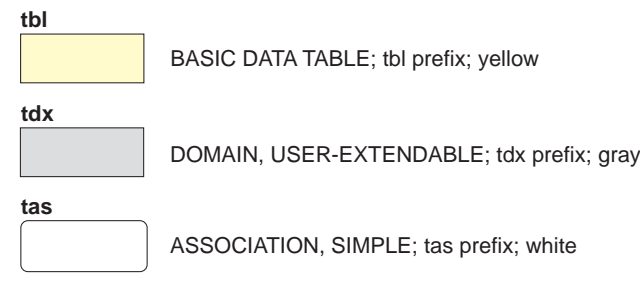

Table and Relationship Symbols

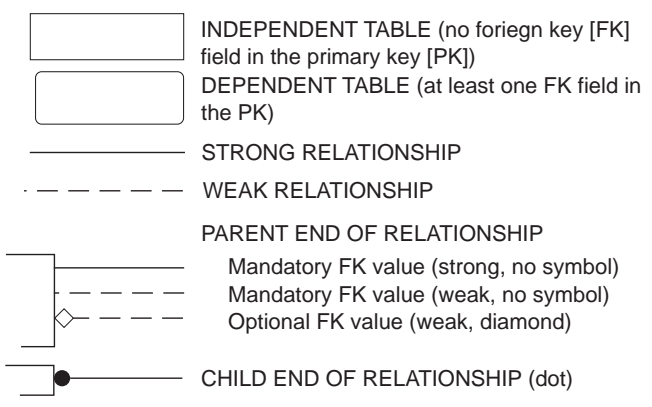

\section{Field Property Indicators}

PK PRIMARY KEY (PK) FIELDS ABOVE LINE NON-PK FIELDS BELOW LINE

(FK) FOREIGN KEY FIELD

(20) NUMBERS IN PARENTHESES MAXIMUM NUMBER OF CHARACTERS

Figure 13. System subject area tables, fields, and relationships. 
Additionally, individual Systems can be identified as part of a larger System (a super System) through the hierarchical association of a ParentSystem_ID value in $t d x$ System with a different System record (a selfjoin). Thus, a public-supply network could have a System name and consist of two or more named subSystems. Care should be exercised in developing super Systems because individual Sites may be present in more than one subSystem (shared Sites); duplicates should be resolved when handling Systems made up of other Systems.

Not all aggregates of Sites need to be created as named Systems. With the use of domains and other fields in Site-related tables, any number of useful sets of Sites that meet specific criteria can be assembled, such as those related to UseType, Location (geopolitical and hydrologic), Resource properties, Conveyance actions, and Transfer properties. The use of the System tables is reserved for custom, user-defined aggregates of Sites that are identifiable by a convenient name and that need to be manipulated as a group.

\section{Address Subject Area}

The Address subject area focuses on the tblAddress table and its related entities. The Address subject area diagram is shown in figure 14. The tblAddress table provides a single place to store Address information for Sites and Owners in NJWaTr. Each Address record has postal fields and is classified by a selection from the $t d s A d d r e s s T y p e$ domain. The $t d s A d d r e s s T y p e$ domain currently contains three values to accommodate the types of Addresses anticipated: "Street," "Mailing," and "Street and Mailing." One or more types of Addresses for each Site or Owner can be stored by pairing an Address of a defined type with a Site or Owner using the tasSiteAddress and tasOwnerAddress association tables. Storing Address information in this way provides data-storage efficiency for the database. Because only Addresses that are known are stored, different types of Addresses can be associated with single Sites or Owners; individual Addresses can serve more than one Site and Owner as appropriate (sharing an Address_ID); and fields for storing this optional information are not needed in the tblSite or tblOwner tables themselves.

\section{DataSource Subject Area}

The DataSource subject area focuses on the tdxDataSource domain table and its related entities. The DataSource subject area diagram is shown in figure 15. The tdxDataSource table is a user-extended domain and provides NJWaTr with a single place to store information about the original source of infomation for records in various entities. Each DataSource has an Owner (Owner_ID is a FK) and a DataSource text descriptor field. Because a DataSource has an Owner, some records in the tblOwner table may be entered only to support a DataSource and not for association with Sites or Conveyances. The DataSourceMemo field is provided in $t d x$ DataSource for optional notes about each DataSource. DataSource is a mandatory FK (DataSource_ID) that identifies the original source of data stored for Site and Resource User-Defined Details (in the tadSiteDetail and tadResourceDetail tables, respectively), for Permit and Alias Labels (tdxPermitLabel and tdxAliasLabel, respectively), and for Transfer/Volumes (tblVolume). The tdxDataSource table can be used to identify and retrieve records that are associated with a particular DataSource or its parent Owner.

\section{Permit Subject Area}

The Permit subject area focuses on the tblPermit table and its related entities. The Permit subject area diagram, shown in figure 16, illustrates that the generic tblPermit table is linked through associative entities to Sites and Owners. The identification of Sites and Owners by their agency-supplied Permit codes was a 


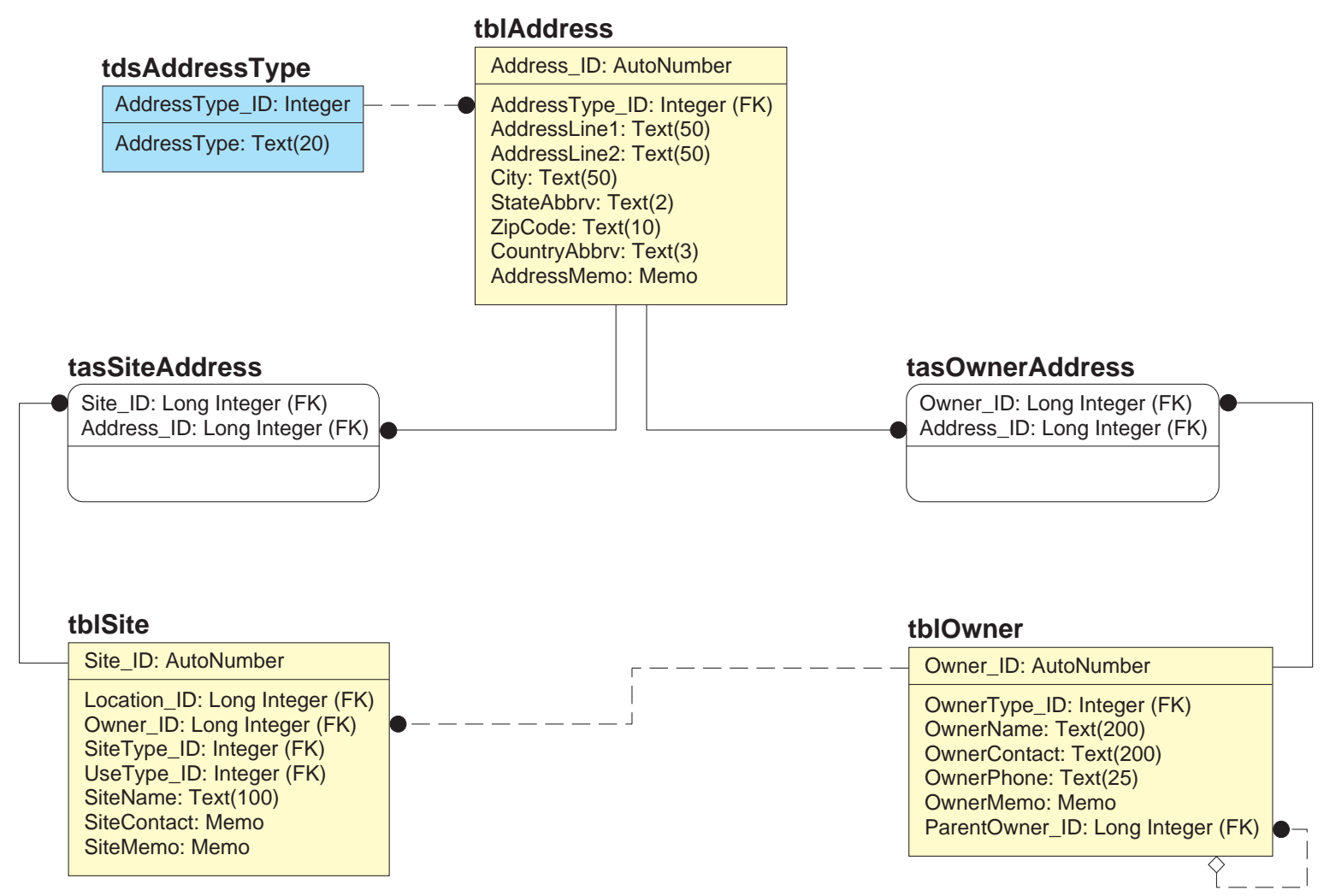

\section{EXPLANATION}

\section{Functional Table Types}

tbl

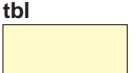

tds

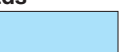

tas

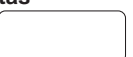

BASIC DATA TABLE; tbl prefix; yellow

DOMAIN, STATIC; tds prefix; blue

ASSOCIATION, SIMPLE; tas prefix; white
Table and Relationship Symbols

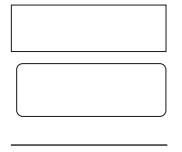

NDEPENDENT TABLE (no foriegn key [FK]

field in the primary key [PK])

DEPENDENT TABLE (at least one FK field in

the PK)

STRONG RELATIONSHIP

- - - - WEAK RELATIONSHIP

PARENT END OF RELATIONSHIP

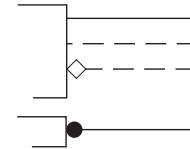

Mandatory FK value (strong, no symbol)

Mandatory FK value (weak, no symbol)

Optional FK value (weak, diamond)

CHILD END OF RELATIONSHIP (dot)
Field Property Indicators

PRIMARY KEY (PK) FIELDS ABOVE LINE NON-PK FIELDS BELOW LINE

(FK) FOREIGN KEY FIELD

(20) NUMBERS IN PARENTHESES INDICATE TEXT FIELD MAXIMUM NUMBER OF CHARACTERS

Figure 14. Address subject area tables, fields, and relationships. 
tdxDataSource

tblOwner

Owner_ID: AutoNumber

OwnerType_ID: Integer NOT NULL (FK)

OwnerName: Text(200) NOT NULL

OwnerContact: Text(200)

OwnerPhone: Text(25)

OwnerMemo: Memo

ParentOwner_ID: Long Integer (FK)

$P_{--1}^{-}$

Owner_ID: Long Integer NOT NULL (FK)

DataSource: Text(75) NOT NUL

DataSourceMemo: Memo

\section{tadSiteDetail}

Site ID: Long Integer NOT NULL (FK)

SiteDetailabel_ID. Long Integer NOT NULL (FK)

SiteDetailEffectiveDate: Date/Time NOT NULL

SiteDetailEndingDate: Date/Time

DataSource_ID: Long Integer NOT NULL (FK)

Timelnterval ID: Integer NOT NULL (FK)

SiteDetailValue: Text(50) NOT NULL

SiteDetailMemo: Memo

\section{tadResourceDetail}

Resource_ID: Long Integer NOT NULL (FK)

ResourceDetaill

ResourceDetailEffective Late: Date/Time NOT NULL

ResourceDetailEndingDate: Date/Time

DataSource_ID: Long Integer NOT NULL (FK)

ResourceDetail: Text(50) NOT NULL

ResourceDetailMemo: Memo

\section{EXPLANATION}

\section{Functional Table Types}

tbl

\begin{tabular}{l}
\hline BASIC DATA TABLE; tbl prefix; yellow \\
$\mathbf{t d x}$ DOMAIN, USER-EXTENDABLE; tdx prefix; gray \\
\hline
\end{tabular}

tad

ASSOCIATION, WITH DATA; tad prefix; green

\section{Table and Relationship Symbols}

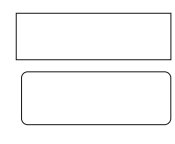

INDEPENDENT TABLE (no foriegn key [FK] field in the primary key [PK])

DEPENDENT TABLE (at least one FK field in

the PK)

STRONG RELATIONSHIP

- - - - - WEAK RELATIONSHIP

PARENT END OF RELATIONSHIP

Mandatory FK value (strong, no symbol)

Mandatory FK value (weak, no symbol)

Optional FK value (weak, diamond)

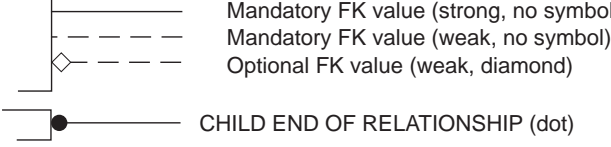

\section{tblVolume}

Volume_ID: AutoNumber

Transfer_ID: Long Integer NOT NULL (FK)

Staff_ID: Long Integer NOT NULL (FK)

DataSource_ID: Long Integer NOT NULL (FK)

VolumeMethod_ID: Long Integer NOT NULL (FK)

RawVolumeValue: Double NOT NULL

VolumeUnit_ID: Long Integer NOT NULL (FK)

sDefaultVolume: Yes/No NOT NULL

VolumeMemo: Memo

\section{tdxAliasLabel}

AliasLabel_ID: AutoNumber

DataSource_ID: Long Integer NOT NULL (FK) AliasSource: Text(75)

AllasLabel: Text(50) NOT NULL

AliasLabelMemo: Memo

\section{tdxPermitLabe}

PermitLabel_ID: AutoNumber

DataSource_ID: Long Integer NOT NULL (FK)

PermitLabel: Text(50) NOT NULL

PermitLabelMemo: Memo

\section{Field Property Indicators}

PK PRIMARY KEY (PK) FIELDS ABOVE LINE NON-PK FIELDS BELOW LINE

(FK) FOREIGN KEY FIELD

(20) NUMBERS IN PARENTHESES INDICATE TEXTFIELD MAXIMUM NUMBER OF
CHARACTERS

Figure 15. DataSource subject area tables, fields, and relationships. 


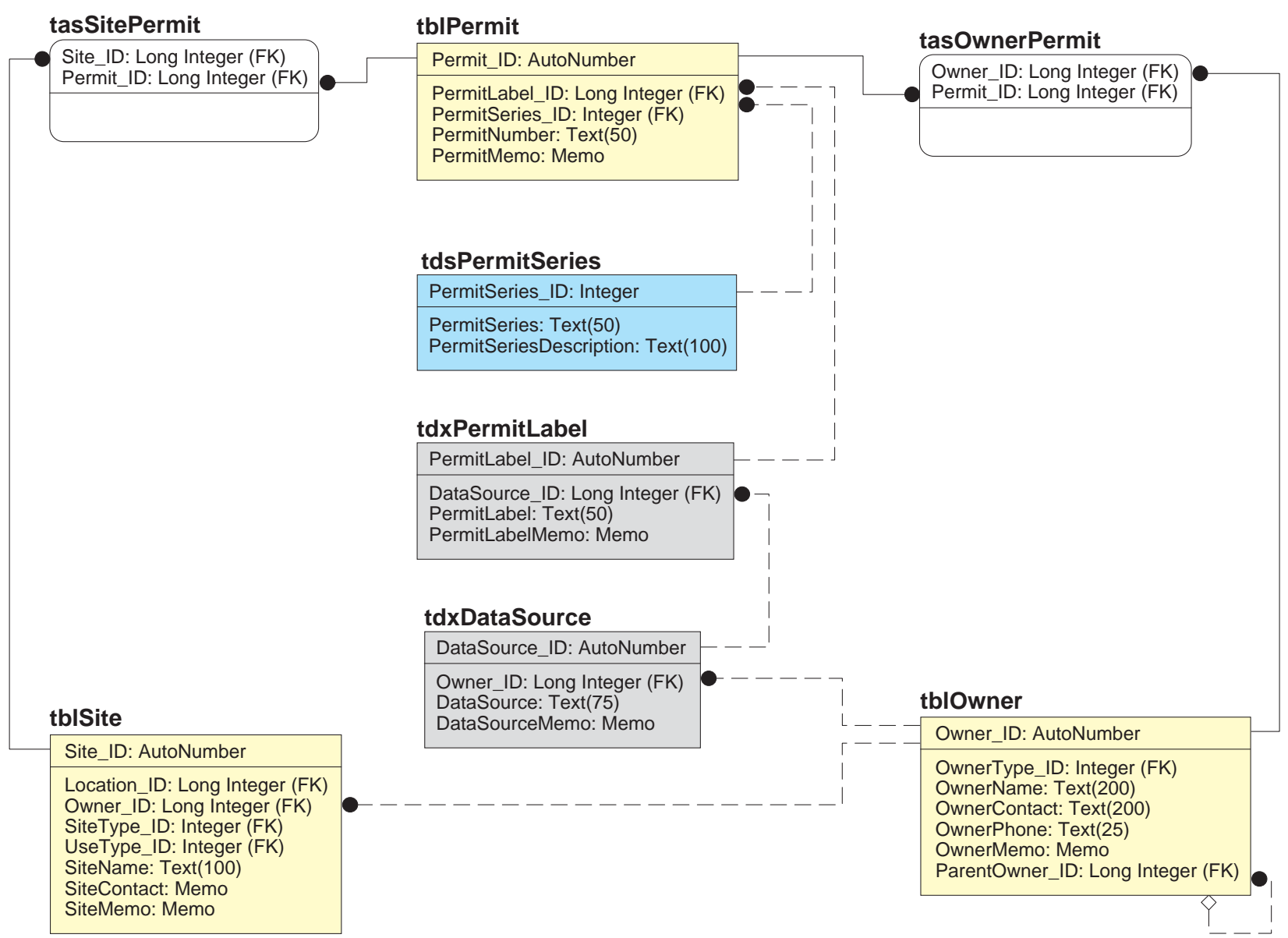

\section{Functional Table Types}

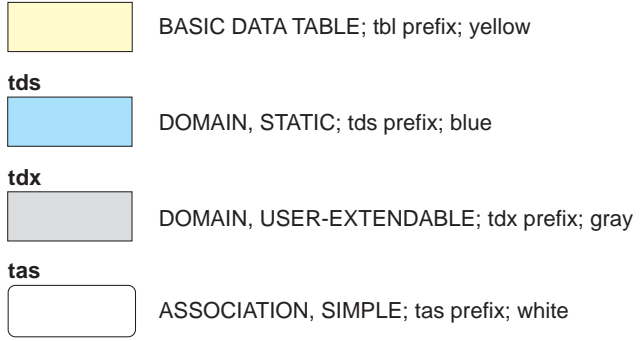

\section{EXPLANATION}

Table and Relationship Symbols

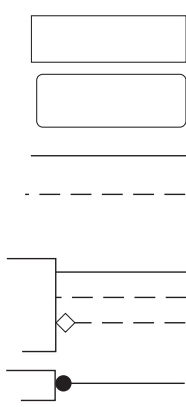

NDEPENDENT TABLE (no foriegn key [FK] field in the primary key [PK])

DEPENDENT TABLE (at least one FK field in the PK)

\section{STRONG RELATIONSHIP}

WEAK RELATIONSHIP

PARENT END OF RELATIONSHIP

Mandatory FK value (strong, no symbol)

Mandatory FK value (weak, no

FK value (weak, diamond)

\section{Field Property Indicators}

PK PRIMARY KEY (PK) FIELDS ABOVE LINE NON-PK FIELDS BELOW LINE

(FK) FOREIGN KEY FIELD

(20) NUMBERS IN PARENTHESES
INDICATE TEXT FIELD INDICATE TEXT FIELD CHARACTERS

Figure 16. Permit subject area tables, fields, and relationships. 
high-priority requirement for the database. Permits for Sites, for example, allow Site information to be displayed and reported using alternative state, Federal, or local regulatory codes familiar to users. In addition, Permit codes can be useful during batch loading of data through a data-entry application.

Permit storage and identification in NJWaTr is implemented after the fashion of Aliases in the NEWUDS model; Aliases also are present in the NJWaTr model and will be discussed separately. A single tblPermit table serves the Permit storage and identification function for all entities. This approach offers the advantage of having no Null values in any field used for Permit assignment, keeping the number of tables and fields involved in Permit assignment and identification to a minimum, and allowing any number of Permits for any number of entities to be implemented using the same structure. Because the tblPermit table only contains Permits that are purposely defined, Sites and Owners that do not have or need a Permit assignment do not have a record in that table.

The Permit structure works by keeping all Permit values (the assigned name or code) in one table along with a label selection from the $t d x$ PermitLabel domain, which includes the source of the Permit (DataSource whose Permit code is being applied). Sites and Owners are linked to the tblPermit table through association tables, where an individual entity instance is paired with an individual Permit. The association tables provide a rapid method for checking to see whether a Permit exists for a given item (by searching for the item's _ID value), whereas the tdxPermitLabel table allows filtering for Permits from a specific DataSource or for kinds of Permits that are assigned a specific PermitLabel value.

In the table tblPermit, each Permit is identified by a unique Permit_ID. Each Permit is defined by a standardized set of attributes: a PermitSeries, PermitLabel, and PermitNumber. The PermitSeries is a selection from a static domain, $t d s$ PermitSeries, that stores a list of water allocation, safe drinking-water, and New Jersey Pollutant Discharge Elimination System (NJPDES) permit series codes used to group Sites in New Jersey (Hoffman and Lieberman, 2000). A PermitMemo field is included for recording optional notes about individual Permits when necessary. The PermitLabel is supplied by the tdxPermitLabel domain table, which also holds a FK link to the tdxDataSource domain. The tdxPermitLabel table also includes a memo field, PermitLabelMemo, to record optional descriptions of abbreviated labels or to provide explanations for a label.

Association tables are used to link a Permit to other tables that need Permit assignment. Thus, tasSitePermit links a Site_ID to a Permit_ID, and tasOwnerPermit links an Owner_ID to a Permit_ID. Additional associations could be added later for other entities that are not currently provided with Permit assignments. Each Permit record will typically serve a single associate but can apply to more than one if that is a valid assignment, and the Permit is not limited to any one entity type because it does not store any associate information directly (the linkage is handled in the tas association tables). Single items also can have as many Permits as needed.

A hypothetical example of defining a Permit in NJWaTr can be illustrated with an Owner's agricultural withdrawal Permit. A permit is issued to farmer John Smith for surface-water withdrawals of more than 100,000 gallons per day to serve his large cranberry farm operation. The permit is issued by the Bureau of Water Allocation (BWA) of the NJDEP. Farmer Smith's permit designation is "BWA XYZ0001." In NJWaTr, the Owner_ID for "Smith, John" would be connected to the associated Permit (as Permit_ID) through the tas OwnerPermit table. Appropriate field values for defining the Permit could be as follows:

Owner in tblOwner = "Smith, John" (for an Owner Permit, the Owner here is the person/organization to whom the permit is issued),

DataSource in tdxDataSource = "Bureau of Water Allocation" (issues the allocation permit; the Owner of the DataSource is the NJDEP), 
PermitLabel in tdxPermitLabel = "BWA" (label for a BWA permit),

PermitNumber in tblPermit = "XYZ0001" (uniquely identifies John Smith's BWA permit), and

PermitSeries in tdsPermitSeries = "Agricultural certifications" (surface- and ground-water withdrawals for agricultural use $>100,000$ gallons per day).

Using this example, if all BWA Permits used the same PermitLabel value, it would be possible to construct a query to find all BWA-Permitted Owners or to locate an individual Owner if the Permit number was known.

Permits can be retrieved as a group on the basis of the source or label to supply a different name field to the associated entity. For example, the Permit structure and data can be manipulated through a query to automatically create fields for each label for each Permitted entity. An example of using a crosstab query to link Permits directly to the Owner table as extended attributes is given in the section "Construction and Use of Views."

Using the DataSource and PermitLabel fields judiciously, it is possible to store and retrieve an unlimited number of regulatory coding schemes (by agency or department) and use them in place of the formal names given in the associated entities. This is much more powerful than setting up separate fields for specific coding schemes. It is expected that over time, however, examination of the tblPermit table will indicate which Permits are truly needed as fields within the Site and Owner entities and that the tblPermit table then can be used to transfer values from those fields to the other tables directly, such as making a BWA field in the tblSite table to hold Site-specific BWA permit numbers.

One more advantage of storing Permits is that the codes stored could be used to link to other databases. In the discharge Permit example above, because the PermitNumber "XYZ0001" would be the same identification used in the NJDEP BWA database, comparisons of information, retrievals, and imports from other databases made using the Permit code would be facilitated.

\section{Alias Subject Area}

The Alias subject area focuses on the tblAlias table and its related entities. The Alias subject area diagram, shown in figure 17, illustrates that the generic tblAlias table is linked through associative entities to Sites, Resources, and Conveyances. Aliases are distinct from Permits in that a Permit code is an officially assigned regulatory identifier, whereas an Alias represents all other alternative naming schemes (such as a local name). Aliases for Sites, for example, would allow Site information to be displayed and reported using alternative state, Federal, or local names familiar to users or by their unique identifiers found in other databases. Aliases, like Permit numbers, also can be useful during batch loading of data through a data-entry application.

Initially, some Alias codes were created using separate fields within entities bearing the name of the alias scheme. The current system uses a single tblAlias table to serve the alias function for all entities. The same advantages as with the Permit entity apply: no Null values are allowed in any field used for alternative naming schemes; the number of tables and fields involved in providing complete alias services are kept to a minimum; and any number of naming schemes for any number of entities are implemented using the same 
tblAlias

Alias_ID: AutoNumber

AliasLabel_ID: Long Integer (FK)

AliasValue: Text(50)

AliasMemo: Memo

\section{tdxAliasLabe}

AliasLabel_ID: AutoNumber

DataSource_ID: Long Integer (FK)

AliasSource: Text(75)

AliasLabel: Text(50)

AliasLabelMemo: Memo

\section{tdxDataSource}

DataSource_ID: AutoNumber

Owner_ID: Long Integer (FK)

DataSource: Text(75)

DataSourceMemo: Memo
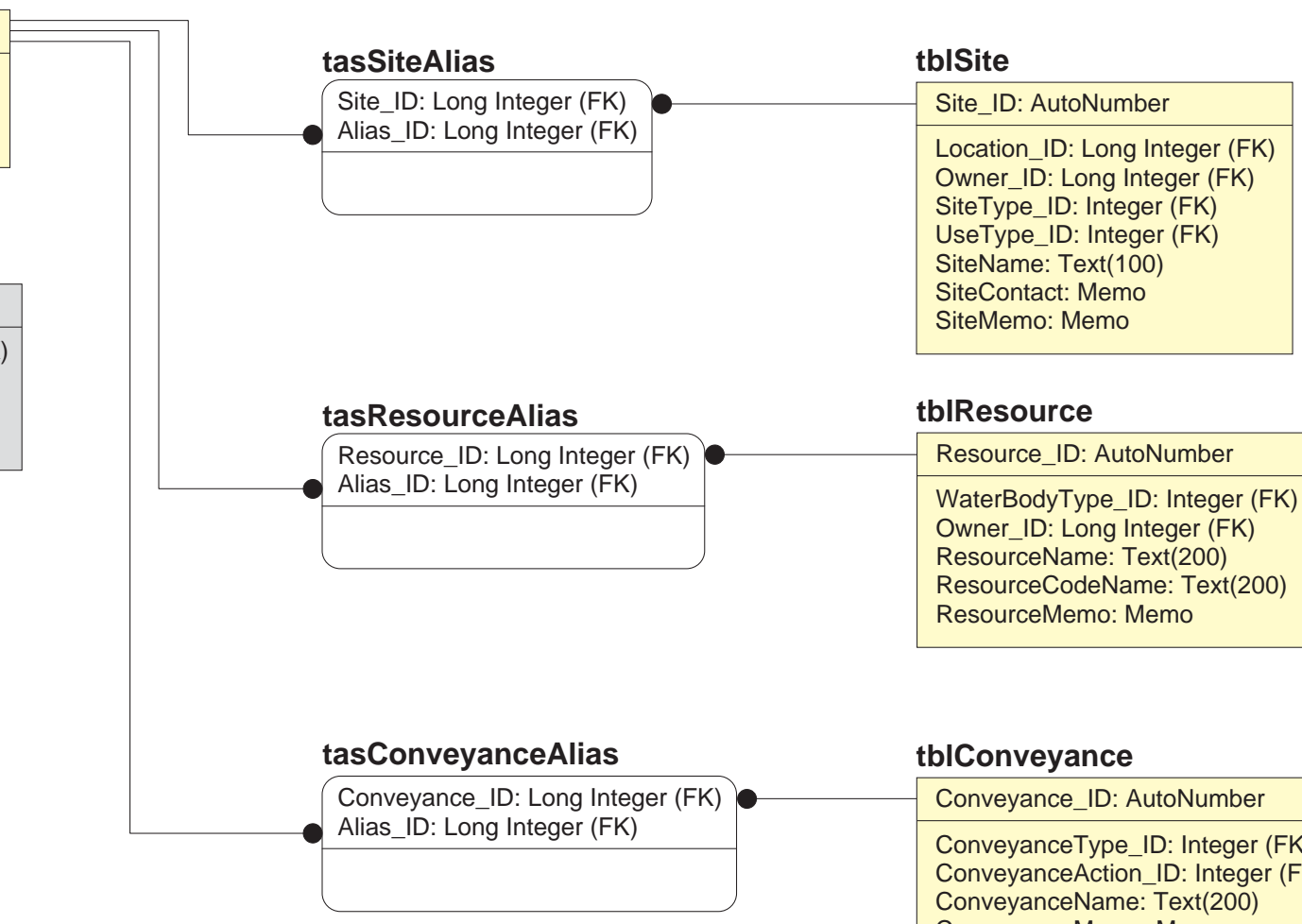

tblResource

Resource_ID: AutoNumber

WaterBodyType_ID: Integer (FK) Owner_ID: Long Integer (FK)

ResourceName: Text(200)

ResourceCodeName: Text(200) ResourceMemo: Memo

\section{EXPLANATION}

Functional Table Types

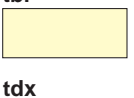

tas

ASSOCIATION, SIMPLE; tas prefix; white
Table and Relationship Symbols

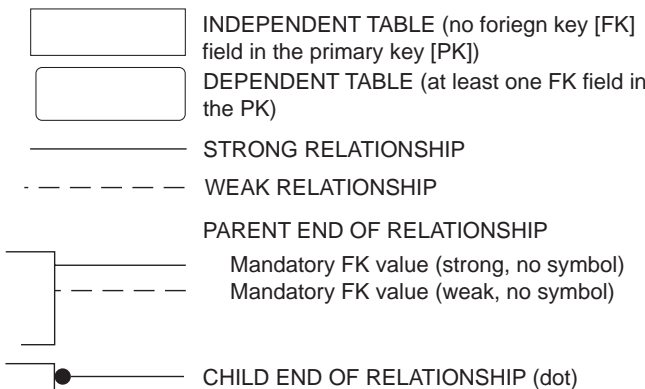

Field Property Indicators

PK PRIMARY KEY (PK) FIELDS ABOVE LINE NON-PK FIELDS BELOW LINE

(FK) FOREIGN KEY FIELD

(20) NUMBERS IN PARENTHESES
INDICATE TEXT FIELD MAXIMUM NUMBER OF CHARACTERS

Figure 17. Alias subject area tables, fields, and relationships. 
structure. Because the tblAlias table contains only Aliases that are purposely defined, Sites, Resources, and Conveyances that do not have or need an Alias do not have a record in that table.

The Alias structure works by keeping all Alias values (the alternative name or code) in one table, along with a label selection from the tdxAliasLabel domain which includes the source of the Alias (whose naming scheme is being used). Each entity that requires alias services is linked to the tblAlias table through an association table, where an individual entity record is paired with an individual Alias. The association table provides a rapid method for checking to see whether an Alias exists for a given item (by searching for the item's_ID value), whereas the tdxAliasLabel table allows filtering for Aliases from a specific DataSource or assigned a specific AliasLabel.

In the table tblAlias, each Alias is identified by a unique Alias_ID. Each Alias is defined by a standardized set of attributes: an AliasValue, AliasLabel, and AliasSource. An AliasMemo field is included for recording optional notes about individual Aliases when necessary. The AliasLabel and AliasSource are supplied by the $t d x$ AliasLabel domain table, which also holds a link to the tdxDataSource domain. The tdxAliasLabel table also includes a memo field, AliasLabelMemo, to record optional descriptions of abbreviated labels or to provide explanations for a label.

Association tables are used to link an Alias to other tables that need Alias assignment. Thus, tasSiteAlias links a Site_ID to an Alias_ID; tasResourceAlias links a Resource_ID to an Alias_ID; and tasConveyanceAlias links a Conveyance_ID to an Alias_ID. Additional associations could be added later for other entities that are not currently provided with alias services. Each Alias record will typically serve a single associate but can apply to more than one. An Alias record is not limited to any one entity type because it does not store any associate information directly (the linkage is handled in the tas association tables). Single items also can have as many Aliases as needed.

Aliases can be retrieved as a group on the basis of the source or label to supply a different name field to the associated entity. For example, the Alias structure and data can be manipulated through a query to automatically create fields for each label for each entity associated with an Alias. The example of using a crosstab query to link Permit numbers directly to the Owner table as extended attributes given in the section, "Construction and Use of Views," also is applicable to Aliases.

Using the AliasSource and AliasLabel fields judiciously, it is possible to store and retrieve an unlimited number of alternative coding systems (by agency, office, or individual) and use them in place of the formal names given in the associated entities. This is much more powerful than setting up separate fields for specific coding schemes. It is expected that over time, however, examination of the tblAlias table will indicate which Aliases are truly needed as fields within the tblSite, tblConveyance, and tblResource data tables. The tblalias table then can be used to transfer values from those fields to the other tables directly.

\section{User-Defined Details}

The User-Defined Details subject area describes the entity and relationship components that serve the unknown attribute storage needs of several entities. The User-Defined Details subject area diagram is shown in figure 18. Several specific, optional attributes of various entities in the database were added as fields during the development of NJWaTr and later removed. Some of these fields became domains, whereas others were removed because it was determined that they applied only to a small subset of the table's records or would be used infrequently. A general method was needed to store specific kinds of information that are either optional or not known in advance and that could not be designed directly into the table structures. The strategy for storing such data resulted in User-Defined Detail structures in NJWaTr. 


Site_ID: AutoNumber
Location_ID: Long Integer (FK)
Owner_ID: Long Integer (FK)
Siteype_ID: Integer (FK)
UseType_ID: Integer (FK)
SiteName: Text(100)
SiteContact: Memo
SiteMemo: Memo

Site_ID: Long Integer (FK)

SiteDetailLabel ID: Long Integer (FK) SiteDetailEffectiveDate: Date/Time

SiteDetailEndingDate: Date/Time Timelnterval ID: Integer (FK) SiteDetailValue: Text(50) SiteDetailMemo: Memo

\section{tadResourceDetail}

Resource_ID: AutoNumber WaterBodyType_ID: Integer (FK) Owner_ID: Long Integer (FK)

ResourceCodeName: Text(200)

ResourceMemo: Memo

ResourceDetail: Text(50)

Resource ID: Long Integer (FK)

ResourceDetailLabel_ID: Long Integer (FK)

ResourceDetailEffectiveDate: Date/Time

ResourceDetailEndingDate: Date/Time

DataSource_ID: Long Integer (FK)

ResourceDetailMemo: Memo

\section{tblVolume}

tadVolumeDetail

Volume_ID: AutoNumber

Transfer_ID: Long Integer (FK)

DataSource ID: Long Integer (FK)

VolumeMethod_ID: Long Integer (FK)

RawVolumeValue: Double

VolumeUnit_ID: Long Integer (FK)

IsDefaultVolume: Yes/No

VolumeMemo: Memo

\section{Volume_D: Long Integer (FK)}

VolumeDetailLabel_ID: Long Integer (FK)

VolumeDetail: Text(50)

VolumeDetailMemo: Memo

\begin{tabular}{|l|l|} 
tdxSiteDetailLabel \\
\hline & SiteDetailLabel_ID: AutoNumber \\
\hline SiteDetailCategory_ID: Long Integer (FK) \\
SiteDetailLabel: : Text(50) \\
IsNumericDetail: Yes/No \\
SiteDetailUnit: Text(50) \\
SiteDetailLabelMemo: Memo
\end{tabular}

SiteDetailLabelMemo: Memo

\section{tdsTimelnterval}

- - Timelnterval_ID: Integer

Timelnterval: Text(25)

\section{tblConveyance}

Conveyance_ID: AutoNumber

ConveyanceType_ID: Integer (FK)

ConveyanceAction_ID: Integer (FK)

ConveyanceName: Text(200)

ConveyanceMemo: Memo

Functional Table Types
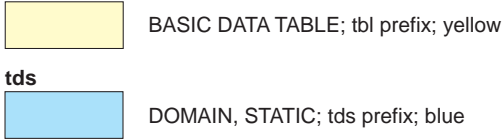

DOMAIN, STATIC; tds prefix; blue

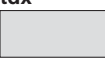

DOMAIN, USER-EXTENDABLE; tdx prefix; gray

ASSOCIATION, WITH DATA; tad prefix; green

\section{tadConveyanceDetai}

Conveyance ID: Long Integer (FK)

ConveyanceDetail: Text(50)

ConveyanceDetail: Text(50)
ConveyanceDetailMemo: Memo

\section{tdxResourceDetailLabel}

ResourceDetailLabel_ID: AutoNumber

ResourceDetaillabel: Text(50)

ResourceDetailLabelMemo: Memo

EXPLANATION

Table and Relationship Symbols

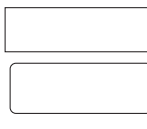

INDEPENDENT TABLE (no foriegn key [FK] ield in the primary key [PK])

DEPENDENT TABLE (at least one FK field in

WEAK RELATIONSHIP

PARENT END OF RELATIONSHIP

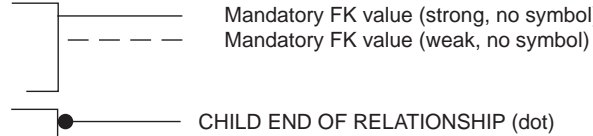

tdxDataSource

DataSource_ID: AutoNumber

Owner_ID: Long Integer (FK)

DataSource: Text(75)

DataSourceMemo: Memo

Figure 18. User-Defined Detail subject area tables, fields, and relationships.

Field Property Indicators

PK PRIMARY KEY (PK) FIELDS ABOVE LINE NON-PK FIELDS BELOW LINE

(FK) FOREIGN KEY FIELD

(20) NUMBERS IN PARENTHESES INDICATE TEXT FIELD MAXIMUM NUMBER OF 
A User-Defined Detail structure uses a tad associative entity to join a specific instance of a core element with a label that describes some detail about it and stores the detail value using that label. Thus, labels can be created as needed for particular detail value types and reused for other associates. As with Alias labels, careful consideration and occasional reevaluation of the detail labels can provide a mechanism for retrieving sets of similar types of information and for refining searches and aggregations of data in controlled ways. NJWaTr contains User-Defined Detail structures for Sites, Conveyances, Volumes, and Resources.

Many details about the various types of Sites in the NJWaTr database can be tracked. Site UserDefined Details can include label values such as "Population served" for public-supply distribution systems or wastewater-collection systems, "Status" to store the operational status of a well or treatment plant, "Employees" to store the number of employees at an industrial facility, "Kilowatts generated" to store the power provided by a nuclear facility, or "Cost" to store the amount charged for water by a purveyor.

User-Defined Details about Sites can change over time, and temporal fields are needed to define the periods of time covered by the detail values stored. The tadSiteDetail table associates a specific Site_ID with a specific SiteDetailLabel_ID and a specific SiteDetailEffectiveDate to form its PK. This complex PK allows the same detail Label to apply to a Site for different non-overlapping periods of time, thus allowing changes in detail values to be tracked over time (for example, the population served by a wastewatercollection operation). The non-PK fields in tadSiteDetail are the SiteDetailEndingDate (if known), the SiteDetailValue itself, a SiteDetailMemo for optional notes about the detail value, and through FKs, the detail DataSource and TimeInterval classification to which the detail applies.

The $t d x$ SiteDetailLabel domain is nested within the $t d x$ SiteDetailCategory domain for general detaillabel classification information. The $t d x$ SiteDetailLabel domain includes a label field, a unit field, and a memo field for notes. A field named IsNumericDetail is used to identify SiteDetailLabels associated with any SiteDetailValue that can be converted to a numeric data type for use in mathematical manipulations (for example, the number of employees at industrial Sites).

Resource User-Defined Details (tadResourceDetail) are similar to Site Details in that they are datebounded and have a DataSource associated with them. Examples of details that could be stored for a Resource are depth or acreage of a reservoir and fisheries classification information.

User-Defined Details about Transfer Volumes (tadVolumeDetail) have a DataSource but are not date-bounded. The structure initially was provided to allow a mechanism to store information on accuracy until a more suitable method was identified. In that case, "Accuracy" would be a VolumeDetailLabel value, and the accuracy to be associated with a particular RawVolumeValue would be entered as the VolumeDetail value. Other labels can be developed as needed.

User-Defined Details for a Conveyance (tadConveyanceDetail) are not date-bounded and do not have a DataSource. Conveyance details could include construction date, material, capacity, and head type (pumped or gravity fed).

\section{OPERATIONAL ISSUES AND PROCEDURES}

The use of NJWaTr for water-use data requires an understanding of the basic data model, details of the data elements, and the operational aspects of working with data in that specific model design. Operational considerations include (1) creation of indexes in an individual implementation of the MS Access database; (2) restrictions on table loading order due to table dependencies and data protection settings, such 
as forcing selection of classification terms from domain tables; (3) automatic updating of fields from other data to facilitate uniformity; and (4) simplification of multi-table structures for handling and presenting data. Several operational strategies have been developed using queries that are stored in the NJWaTr database as named Structured Query Language (SQL) statements.

\section{Indexes}

An index is a separate physical object within a relational database. It stores a sorted list of values from one or a combination of fields with pointers to individual data records that contain those values. An index is used by a database engine to rapidly identify the location of specific records in tables by use of the indexed values; it can significantly increase performance when querying or reporting from a database. A unique index also can be created for one or more fields to ensure the uniqueness of values as an alternate PK or for other purposes. Therefore, management of indexes can affect the operational efficiency and integrity of a database.

NJWaTr is conservative in the general use of indexes. Access automatically creates an index for the PK and any FK fields for each table. The PK is a special type of unique index, and the non-unique index for FK fields facilitates the joining of tables and look-up activity without requiring any textual values that the keys represent to be sorted or indexed. Because NJWaTr follows the standard of using information-neutral surrogate key fields as a table's PK, all domain ( $t d s$ and $t d x)$ and basic data $(t b l)$ tables also have been equipped with one unique index to help prevent inadvertent duplication of data. For example, the table tblOwner has, in addition to its PK (Owner_ID) and FK (OwnerType_ID) indexes, a unique index for the required OwnerName field; the name is protected by the unique index and cannot be duplicated within the table. Some unique indexes are only partially enforced by design, such as one for the ResourceCodeName in the tblResource table; that field is optional and used primarily when there is duplication in the

ResourceName field. The ResourceCodeName index is used to prevent duplication of entered values while ignoring records where no entry was made (a Null value). Associative entities have the uniqueness of each record guaranteed by the structure of the $\mathrm{PK}$ and do not require alternate unique indexes.

The unique indexes can be modified for any implementation of NJWaTr, particularly if it is determined that an index prevents actual unique records from being added. Such restrictive indexes should be changed or removed. The creation of other indexes should be considered for any fields or combinations of fields that could be used for grouping, sorting, or filtering NJWaTr data because an index can improve performance in queries and reports. Because the identification of fields that can benefit from an index depends on how the database is used, creation of additional indexes are left to the individual implementations of the database.

\section{Table Loading Order}

Key structures affect table loading order. This information is important for hand-entry of data and for providing structural logic when building a batch loader or user-interface application for NJWaTr. For example, a tblConveyance record should not be established without records for the Sites that will form the Conveyance ends already in the tblSite table. A tblConveyance record then can be added, followed by two entries in tadSiteConveyance to create the From and To Site identities for the Conveyance. All static ( $t d s$ prefix) and many user-extendable $(t d x)$ domains are already populated in the standard NJWaTr database. Basic data tables $(t b l)$ require at least one domain FK, so they cannot be loaded until the domain is populated with needed values. Association tables (tas and tad) are dependent on parent table records by definition, and throughout NJWaTr, the nesting of table keys establishes other loading order requirements. 
As a guide for users, loading order information is summarized for each of the 76 NJWaTr tables in table 1. This information should be used to establish proper data-entry order and conditions. Columns in table 1 include the following:

Loading order - a number representing the position of a table in the overall loading order. All tables with a loading order of 1 may be populated immediately; 2's require at least one table at the 1 level to have been populated with the necessary records; 3 's require at least one table at level 2 to have the necessary records; and so forth. The loading order is a literal value that represents the direct chain of incoming relational dependencies, but the loading notes also should be consulted to determine the actual sequence on the basis of indirect relationships through other entities;

Table name- the name of the table in NJWaTr;

Incoming relationships - parent tables - the number of tables with defined relationships entering the current table and, therefore, providing a FK, along with a list of the parent table names;

Outgoing relationships - child tables - the number of tables that will receive the PK of the current table as a FK through a defined relationship, along with a list of the child table names; and

Note - things to consider before or during the process of adding records to a table; where "Some fields are optional" is noted, consult the data dictionary for details about optional and required fields in a table.

\section{Maintenance Update Queries}

Several queries are provided in the NJWaTr database to perform data maintenance functions and to facilitate manipulation of the data after it has been entered. The queries to perform these functions are run manually or can be triggered to run automatically within an application interface for NJWaTr. The maintenance queries are named with the prefix qrm to distinguish them from other queries stored within the database.

\section{Volume Unit Updates}

The cluster of domain tables centered on tdxVolumeUnit (fig. 9) provide the ability to create VolumeUnit choices for any possible combination of decimal and quantity components, as discussed in the "Transfer/Volume Subject Area" section of this document. A new VolumeUnit is added to the domain by selecting one choice from each of the two tables tdsVolumeUnitDecimal and tdsVolumeUnitTime; an appropriate standard VolumeUnit abbreviation is entered manually in the field VolumeUnitAbbrv. Two remaining fields are provided with default values of "-1" when new VolumeUnit entries are added-

VolumeUnitPhrase and MGConversion. Although this process establishes the basics of the new record in the $t d x$ VolumeUnit table, one additional step is needed to complete the description of the unit in that table.

The new record in the $t d x$ VolumeUnit table must be updated with a standardized component phrase that describes it in the field VolumeUnitPhrase (for example, "thousand cubic feet"), and a conversion factor from the new unit to a MG equivalent must be determined and placed in the field MGConversion. The default value for both VolumeUnitPhrase and MGConversion for newly created VolumeUnits is "-1." The "-1" value serves as a flag for records that have not yet been updated. Replacing the "-1" flag with a valid conversion value and phrase is done using two queries. One query sets things up and the other performs the update. Only the second query must be run. 
Table 1. Table loading order and related information for the New Jersey Water-Transfer Data System (NJWaTr)

Loading

order Table name

1 tdsAddressType

Incoming relationships - parent tables

0 - none

Note: Standard NJWaTr contains 3 types

$1 \quad$ tdsConveyanceActionCategory

0 - none

1 - tds ConveyanceAction

Note: Standard NJWaTr contains 25 category terms

\begin{tabular}{llcc}
\hline 1 & tdsConveyanceType & $0-$ none & $1-$ tblConveyance \\
& Note: Standard NJWaTr contains 7 types & $1-$ tadSiteResource
\end{tabular}

Note: Standard NJWaTr contains 2 New Jersey areas, plus one record (ID $=0$ ) representing an unknown condition, and another (ID=9) representing a Not Applicable condition; must be modified for use outside of the New Jersey area

\begin{tabular}{|c|c|c|}
\hline \multirow[t]{2}{*}{1} & tdsCriticalZone & 1 - tadSiteResource \\
\hline & & $\begin{array}{l}\text { Note: Standard NJWaTr contains } 5 \text { New Jersey zones, plus one record }(I D=0) \text { representing an unknown condition, and } \\
\text { another }(I D=9) \text { representing a Not Applicable condition; must be modified for use outside of the New Jersey area }\end{array}$ \\
\hline \multirow[t]{2}{*}{1} & tdsDroughtRegion & $1-t d s M C D$ \\
\hline & & $\begin{array}{l}\text { Note: Standard NJWaTr contains } 6 \text { New Jersey regions, plus one record }(I D=0) \text { representing a Not Applicable condition; } \\
\text { must be modified for use outside of the New Jersey area }\end{array}$ \\
\hline \multirow[t]{2}{*}{1} & tdsLocationScale & 1 - tblLocation \\
\hline & & Note: Standard NJWaTr contains 7 scales, plus one record $(I D=0)$ representing an unknown condition \\
\hline \multirow[t]{2}{*}{1} & tdsOwnerType & 1 - tblOwner \\
\hline & & Note: Standard NJWaTr contains 6 types, plus one record $(I D=0)$ representing an unknown condition \\
\hline \multirow[t]{2}{*}{1} & tdsPermitSeries & 1 - tblPermit \\
\hline & & $\begin{array}{l}\text { Note: Standard NJWaTr contains } 9 \text { New Jersey Permit Series classifications, plus one record }(\text { ID }=0) \text { representing an } \\
\text { unknown condition; must be modified for use outside of the New Jersey area }\end{array}$ \\
\hline
\end{tabular}


Table 1. Table loading order and related information for the New Jersey Water-Transfer Data System (NJWaTr)—Continued

\begin{tabular}{|c|c|c|c|}
\hline $\begin{array}{l}\text { Loading } \\
\text { order }\end{array}$ & Table name & Incoming relationships - parent tables & Outgoing relationships - child tables \\
\hline \multirow[t]{2}{*}{1} & tdsResourceType & 0 - none & 1 - tdsWaterBodyType \\
\hline & & \multicolumn{2}{|c|}{ Note: Standard NJWaTr contains 6 types, plus one record $(I D=0)$ representing an unknown condition } \\
\hline \multirow[t]{2}{*}{1} & tdsSiteTypeCategory & 0 - none & 1 - tdsSiteTypeSubcategory \\
\hline & & \multicolumn{2}{|c|}{$\begin{array}{l}\text { Note: Standard NJWaTr contains } 6 \text { category terms, plus one record }(I D=0) \text { representing an unknown condition and used } \\
\text { temporarily when loading Site data }\end{array}$} \\
\hline \multirow[t]{2}{*}{1} & tdsState & 0 - none & 2 - tdsCounty, tadLocationState \\
\hline & & \multicolumn{2}{|c|}{$\begin{array}{l}\text { Note: Standard NJWaTr contains } 5 \text { states, plus one record }(I D=0) \text { representing an unknown condition; must be modified for } \\
\text { use with states not contiguous with New Jersey }\end{array}$} \\
\hline \multirow[t]{2}{*}{1} & tdsTimelnterval & 0 - none & 2 - tadSiteDetail, tb/Transfer \\
\hline & & \multicolumn{2}{|l|}{ Note: Standard NJWaTr contains 11 intervals } \\
\hline \multirow[t]{2}{*}{1} & tdsUseGroup & $0-$ none & $1-t d s U s e T y p e$ \\
\hline & & \multicolumn{2}{|c|}{$\begin{array}{l}\text { Note: Standard NJWaTr contains } 7 \text { New Jersey groups, plus one record }(I D=0) \text { representing an unknown condition; must be } \\
\text { modified for use outside of the New Jersey area }\end{array}$} \\
\hline \multirow[t]{2}{*}{1} & tdsVolumeUnitDecimal & 0 - none & $1-t d x$ VolumeUnit \\
\hline & & \multicolumn{2}{|c|}{ Note: Standard NJWaTr contains 10 decimal terms } \\
\hline \multirow[t]{2}{*}{1} & tdsVolumeUnitQuantity & 0 - none & $1-t d x$ VolumeUnit \\
\hline & & \multicolumn{2}{|l|}{ Note: Standard NJWaTr contains 6 quantity terms } \\
\hline \multirow[t]{2}{*}{1} & tdsWaterRegion & 0 - none & 1 - tdsWaterMgmntArea \\
\hline & & \multicolumn{2}{|c|}{$\begin{array}{l}\text { Note: Standard NJWaTr contains } 5 \text { New Jersey regions, plus one record }(I D=0) \text { representing an unknown condition; must be } \\
\text { modified for use outside of the New Jersey area }\end{array}$} \\
\hline \multirow[t]{2}{*}{1} & tdxConveyanceDetailLabel & 0 - none & 1 - tadConveyanceDetail \\
\hline & & \multicolumn{2}{|c|}{ Note: Standard NJWaTr contains 5 labels; populate with appropriate values as needed } \\
\hline
\end{tabular}


Table 1. Table loading order and related information for the New Jersey Water-Transfer Data System (NJWaTr)—Continued

\begin{tabular}{|c|c|c|c|}
\hline $\begin{array}{l}\text { Loading } \\
\text { order }\end{array}$ & Table name & Incoming relationships - parent tables & Outgoing relationships - child tables \\
\hline \multirow[t]{2}{*}{1} & tdxLocationDetMethod & 0 - none & $1-$ tblLocation \\
\hline & & \multicolumn{2}{|c|}{$\begin{array}{l}\text { Note: Standard NJWaTr contains } 10 \text { methods, plus one record }(I D=0) \text { representing an unknown condition; populate with } \\
\text { appropriate values as needed }\end{array}$} \\
\hline \multirow[t]{2}{*}{1} & tdxResourceDetailLabel & 0 - none & 2 - tadResourceDetail, tasWaterBodyDetailLabel \\
\hline & & \multicolumn{2}{|c|}{$\begin{array}{l}\text { Note: Standard NJWaTr contains } 5 \text { labels; populate with appropriate values as needed; labels may be paired with records } \\
\text { from tdsWaterBodyType to create valid combinations in tasWaterBodyDetailLabel }\end{array}$} \\
\hline \multirow[t]{2}{*}{1} & tdxSiteDetailCategory & 0 - none & $1-t d x$ SiteDetailLabel \\
\hline & & \multicolumn{2}{|c|}{ Note: Standard NJWaTr contains 5 category terms; populate with appropriate values as needed } \\
\hline \multirow[t]{2}{*}{1} & tdxStaff & 0 - none & 1 - tb/Volume \\
\hline & & \multicolumn{2}{|c|}{ Note: Can be pre-populated with intended NJWaTr data entry staff; populate with appropriate values as needed } \\
\hline \multirow[t]{2}{*}{1} & tdxSystemType & 0 - none & $1-t d x$ System \\
\hline & & \multicolumn{2}{|c|}{ Note: Standard NJWaTr contains 6 types; populate with appropriate values as needed } \\
\hline \multirow[t]{2}{*}{1} & tdxVolumeDetailLabel & 0 - none & 1 - tadVolumeDetail \\
\hline & & \multicolumn{2}{|c|}{ Note: Standard NJWaTr contains 1 label ("Accuracy"); populate with appropriate values as needed } \\
\hline \multirow[t]{2}{*}{1} & tdxVolumeMethodCategory & 0 - none & $1-t d x$ VolumeMethod \\
\hline & & \multicolumn{2}{|c|}{$\begin{array}{l}\text { Note: Standard NJWaTr contains } 11 \text { category terms, plus one record }(I D=0) \text { representing an unknown condition; populate } \\
\text { with appropriate values as needed }\end{array}$} \\
\hline \multirow[t]{2}{*}{2} & tblAddress & $1-$ tdsAddressType & 2 - tasOwnerAddress, tasSiteAddress \\
\hline & & \multicolumn{2}{|c|}{ Note: Some fields are optional; care should be excercised to avoid duplicate entries having minor spelling variations } \\
\hline \multirow[t]{2}{*}{2} & tb/Location & 2 - tdxLocationDetMethod, tdsLocationScale & $\begin{array}{l}5 \text { - tadLocationCounty, tadLocationHUC, } \\
\text { tadLocationMCD, tadLocationState, tblSite }\end{array}$ \\
\hline & & Note: Location is required for a Site; a default & $=0$ ) representing an unknown condition is provided \\
\hline
\end{tabular}


Table 1. Table loading order and related information for the New Jersey Water-Transfer Data System (NJWaTr)—Continued

\begin{tabular}{|c|c|c|c|}
\hline $\begin{array}{l}\text { Loading } \\
\text { order }\end{array}$ & Table name & Incoming relationships - parent tables & Outgoing relationships - child tables \\
\hline \multirow[t]{2}{*}{2} & tblOwner & 1 - tdsOwnerType & $\begin{array}{l}\text { 6- tasConveyanceOwner, tdxDataSource, } \\
\text { tasOwnerAddress, tasOwnerPermit, tblResource, } \\
\text { tblSite }\end{array}$ \\
\hline & & $\begin{array}{l}\text { Note: Some fields are optional; a default Owne } \\
\text { required for a Site, Resource, and DataS } \\
\text { optional }\end{array}$ & $\begin{array}{l}\text { oresenting an unknown condition is provided; an Owner is } \\
\text { g variation duplicates; ParentOwner_ID is recursive and }\end{array}$ \\
\hline \multirow[t]{2}{*}{2} & tdsConveyanceAction & 1 - tdsConveyanceActionCategory & $1-$ tb/Conveyance \\
\hline & & $\begin{array}{l}\text { Note: Standard NJWaTr contains } 180 \text { actions; } \\
\text { after any new actions are entered }\end{array}$ & te query is needed to create the ConveyanceActionPhrase \\
\hline \multirow[t]{2}{*}{2} & tdsCounty & 1 - tdsState & 2- tadLocationCounty, tdsMCD \\
\hline & & $\begin{array}{l}\text { Note: Standard NJWaTr contains } 177 \text { counties } \\
\text { condition; must be modified for use with }\end{array}$ & $\begin{array}{l}\text { area, plus one record }(I D=0) \text { representing an unknown } \\
\text { us with New Jersey }\end{array}$ \\
\hline \multirow[t]{2}{*}{2} & tdsSiteTypeSubcategory & 1 - tdsSiteTypeCategory & $1-$ tdsSiteType \\
\hline & & $\begin{array}{l}\text { Note: Standard NJWaTr contains } 12 \text { subcateg } \\
\text { used temporarily when loading Site data }\end{array}$ & record $(I D=0)$ representing an unknown condition and \\
\hline \multirow[t]{2}{*}{2} & tdsUseType & 1- tdsUseGroup & 3- tadUseConsumedFraction, tblSite, tadSiteUseType \\
\hline & & $\begin{array}{l}\text { Note: Standard NJWaTr contains } 37 \text { type term } \\
\text { must be modified for use outside of the } 1\end{array}$ & lus one record $(I D=0)$ representing an unknown condition; \\
\hline \multirow[t]{2}{*}{2} & tdsWaterBodyType & $1-$ tdsResourceType & 2- tblResource, tasWaterBodyDetailLabel \\
\hline & & $\begin{array}{l}\text { Note: Standard NJWaTr contains } 13 \text { types, plu } \\
\text { may be paired with records from tdxRes }\end{array}$ & $\begin{array}{l}\text { 0) representing an unknown condition; water body types } \\
\text { tasWaterBodyDetailLabel to create valid combinations }\end{array}$ \\
\hline \multirow[t]{2}{*}{2} & tdsWaterMgmntArea & 1 - tdsWaterRegion & $1-t d s H U C$ \\
\hline & & $\begin{array}{l}\text { Note: Standard NJWaTr contains } 20 \text { New Jers } \\
\text { modified for use outside of the New Jers }\end{array}$ & record $(I D=0)$ representing an unknown condition; must be \\
\hline \multirow[t]{2}{*}{2} & tdxSiteDetailLabel & 1 - tdxSiteDetailCategory & 2 - tadSiteDetail, tasSiteTypeDetailLabel \\
\hline & & $\begin{array}{l}\text { Note: Standard NJWaTr contains } 15 \text { labels; } p \\
\text { from tdsSiteType to create valid combir }\end{array}$ & $\begin{array}{l}\text { iate values as needed; labels may be paired with records } \\
\text { eDetailLabel }\end{array}$ \\
\hline
\end{tabular}


Table 1. Table loading order and related information for the New Jersey Water-Transfer Data System (NJWaTr)—Continued

\begin{tabular}{|c|c|c|c|}
\hline $\begin{array}{l}\text { Loading } \\
\text { order }\end{array}$ & Table name & Incoming relationships - parent tables & Outgoing relationships - child tables \\
\hline \multirow[t]{2}{*}{2} & tdxSystem & $1-$ tdxSystemType & 1 - tasSystemSite \\
\hline & & \multicolumn{2}{|c|}{$\begin{array}{l}\text { Note: System identities may be created before establishing Site records, and the Site associates established in } \\
\text { tasSystemSite later; avoid spelling variation duplicates; ParentSystem_ID is recursive and optional }\end{array}$} \\
\hline \multirow[t]{2}{*}{2} & tdxVolumeMethod & 1 - tdxVolumeMethodCategory & $1-$ tb/Volume \\
\hline & & \multicolumn{2}{|c|}{$\begin{array}{l}\text { Note: Standard NJWaTr contains } 41 \text { methods, plus one record }(I D=0) \text { representing an unknown condition; populate with } \\
\text { appropriate values as needed }\end{array}$} \\
\hline \multirow[t]{2}{*}{2} & tdxVolumeUnit & 2 - tdsVolumeUnitDecimal, tdsVolumeUnitQuantity & $1-$ tblVolume \\
\hline & & \multicolumn{2}{|c|}{$\begin{array}{l}\text { Note: Standard NJWaTr contains } 10 \text { unit terms; create new units as needed by combining decimal and quantity terms; a } \\
\text { maintenance update query is needed to establish the VolumeUnitPhrase and MGConversion values after new unit } \\
\text { records are assembled }\end{array}$} \\
\hline \multirow[t]{2}{*}{3} & tadLocationCounty & 2 - tdsCounty, tb/Location & 0 - none \\
\hline & & \multicolumn{2}{|c|}{ Note: CountyFraction must be entered; IsPrimaryCounty is optional but recommended } \\
\hline \multirow[t]{2}{*}{3} & tadLocationState & 2 - tblLocation, tdsState & 0 - none \\
\hline & & \multicolumn{2}{|c|}{ Note: StateFraction must be entered; IsPrimaryState is optional but recommended } \\
\hline \multirow[t]{2}{*}{3} & tadUseConsumedFraction & $1-$ tdsUseType & 0 - none \\
\hline & & \multicolumn{2}{|c|}{$\begin{array}{l}\text { Note: Use of this table is optional; to use as intended, each UseType to associate with consumptive use should have one } \\
\text { record for each of the } 12 \text { months of the year }\end{array}$} \\
\hline \multirow[t]{2}{*}{3} & tasOwnerAddress & 2 - tblAddress, tblOwner & $0-$ none \\
\hline & & \multicolumn{2}{|c|}{ Note: No special considerations other than verifying the pairing of an Owner with an Address } \\
\hline \multirow[t]{2}{*}{3} & tasWaterBodyDetailLabel & 2 - tdxResourceDetailLabel, tdsWaterBodyType & 0 - none \\
\hline & & \multicolumn{2}{|c|}{$\begin{array}{l}\text { Note: Use of this table is optional; it pairs a WaterBodyType with a ResourceDetailLabel to establish rules for which labels are } \\
\text { appropriate for which water body types }\end{array}$} \\
\hline
\end{tabular}


Table 1. Table loading order and related information for the New Jersey Water-Transfer Data System (NJWaTr)—Continued

\begin{tabular}{|c|c|c|c|}
\hline $\begin{array}{l}\text { Loading } \\
\text { order }\end{array}$ & Table name & Incoming relationships - parent tables & Outgoing relationships - child tables \\
\hline \multirow[t]{2}{*}{3} & tb/Conveyance & 2- tdsConveyanceAction, tdsConveyanceType & $\begin{array}{l}\text { 6- tasConveyanceAlias, tadConveyanceDetail, } \\
\text { tasConveyanceOwner, } \\
\text { tadConveyanceExpectedFraction, } \\
\text { tadSiteConveyance, tb/Transfer }\end{array}$ \\
\hline & & \multicolumn{2}{|c|}{$\begin{array}{l}\text { Note: Sites at Conveyance end points need to be known before creating a Conveyance; a record here should be followed } \\
\text { immediately by establishing the end points in tadSiteConveyance }\end{array}$} \\
\hline \multirow[t]{2}{*}{3} & tblResource & 2 - tblOwner, tdsWaterBodyType & $\begin{array}{l}5 \text { - tadResourceStructure, tadResourceStructure, } \\
\text { tasResourceAlias, tadResourceDetail, } \\
\text { tadSiteResource }\end{array}$ \\
\hline & & \multicolumn{2}{|c|}{$\begin{array}{l}\text { Note: Some fields are optional; a default Resource record }(I D=0) \text { representing an unknown condition is provided; care should } \\
\text { be used to create a unique ResourceCodeName; composite Resources can be separated into components in } \\
\text { tadResourceStructure }\end{array}$} \\
\hline \multirow[t]{2}{*}{3} & tdsHUC & 1 - tdsWaterMgmntArea & 1 - tadLocationHUC \\
\hline & & \multicolumn{2}{|c|}{$\begin{array}{l}\text { Note: Standard NJWaTr contains } 933 \text { 14-digit HUCs for New Jersey, plus one record (ID }=0 \text { ) representing an unknown } \\
\text { condition; must be modified for use outside of the New Jersey area }\end{array}$} \\
\hline \multirow[t]{2}{*}{3} & $t d s M C D$ & 2 - tdsCounty, tdsDroughtRegion & $1-\operatorname{tad} L o c a t i o n M C D$ \\
\hline & & \multicolumn{2}{|c|}{$\begin{array}{l}\text { Note: Standard NJWaTr contains 4,478 MCDs (town equivalent) for the New Jersey area, plus one record }(\text { ID }=0 \text { ) } \\
\text { representing an unknown condition; must be modified for use with states not contiguous with New Jersey }\end{array}$} \\
\hline \multirow[t]{2}{*}{3} & tdsSiteType & 1 - tdsSiteTypeSubcategory & 2 - tb/Site, tasSiteTypeDetailLabel \\
\hline & & \multicolumn{2}{|c|}{$\begin{array}{l}\text { Note: Standard NJWaTr contains } 35 \text { types, plus one record }(I D=0) \text { representing an unknown condition and used temporarily } \\
\text { when loading Site data; site types may be paired with records from tdxSiteDetailLabel to create valid combinations in } \\
\text { tasSiteTypeDetailLabel }\end{array}$} \\
\hline \multirow[t]{2}{*}{3} & tdxDataSource & 1 - tblOwner & $\begin{array}{l}5 \text { - tdxAliasLabel, tdxPermitLabel, tadResourceDetail, } \\
\text { tadSiteDetail, tb/Volume }\end{array}$ \\
\hline & & \multicolumn{2}{|c|}{$\begin{array}{l}\text { Note: Populate with appropriate values as needed; a default DataSource record }(I D=0) \text { representing an unknown condition is } \\
\text { provided; DataSource is required for tblVolume, tdxAliasLabel, tdxPermitLabel, and the detail tables for Sites and } \\
\text { Resources }\end{array}$} \\
\hline \multirow[t]{2}{*}{4} & tadConveyanceDetail & 2 - tb/Conveyance, $t d x$ ConveyanceDetailLabel & 0 - none \\
\hline & & Note: ConveyanceDetail must be entered & \\
\hline
\end{tabular}


Table 1. Table loading order and related information for the New Jersey Water-Transfer Data System (NJWaTr)—Continued

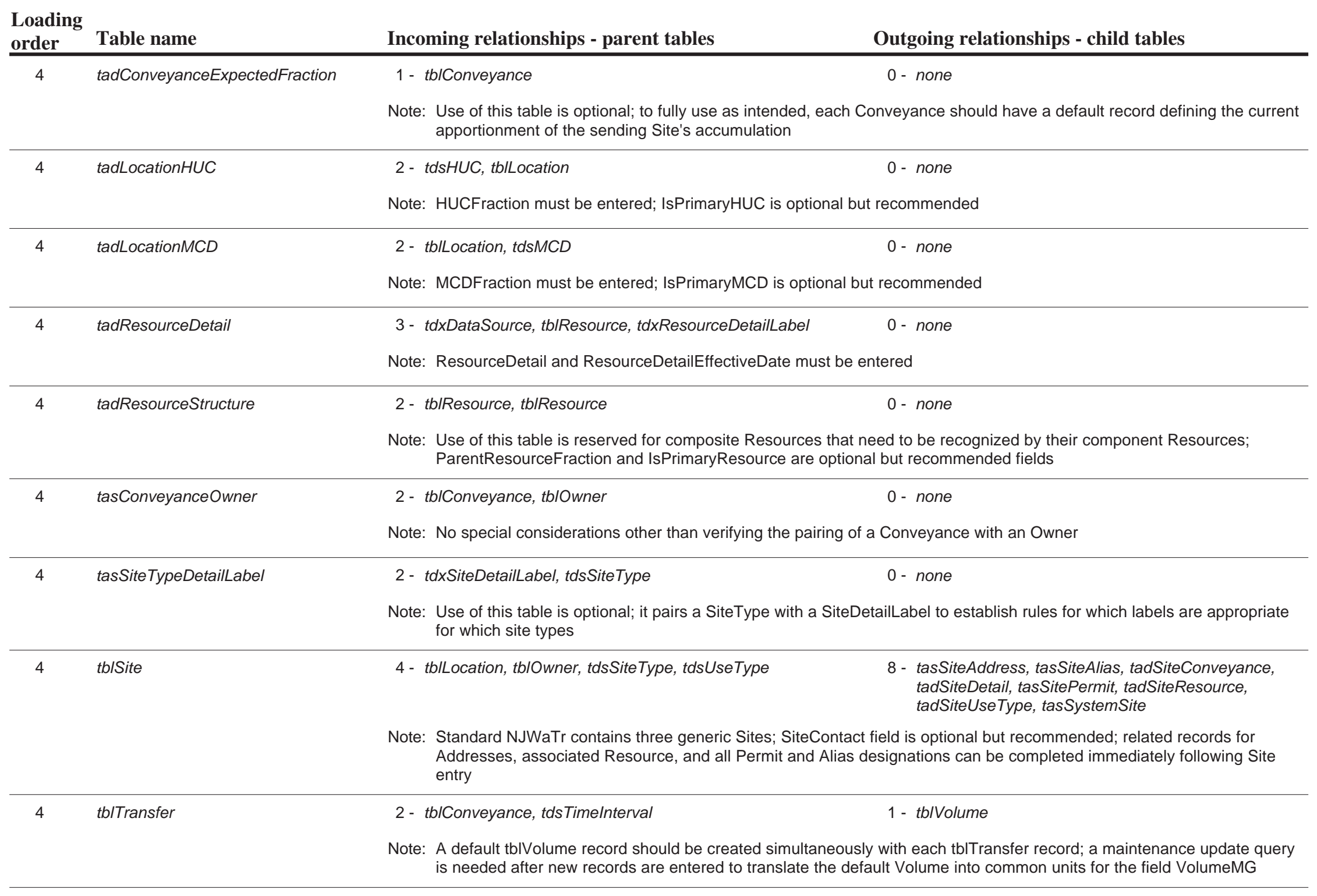


Table 1. Table loading order and related information for the New Jersey Water-Transfer Data System (NJWaTr)—Continued

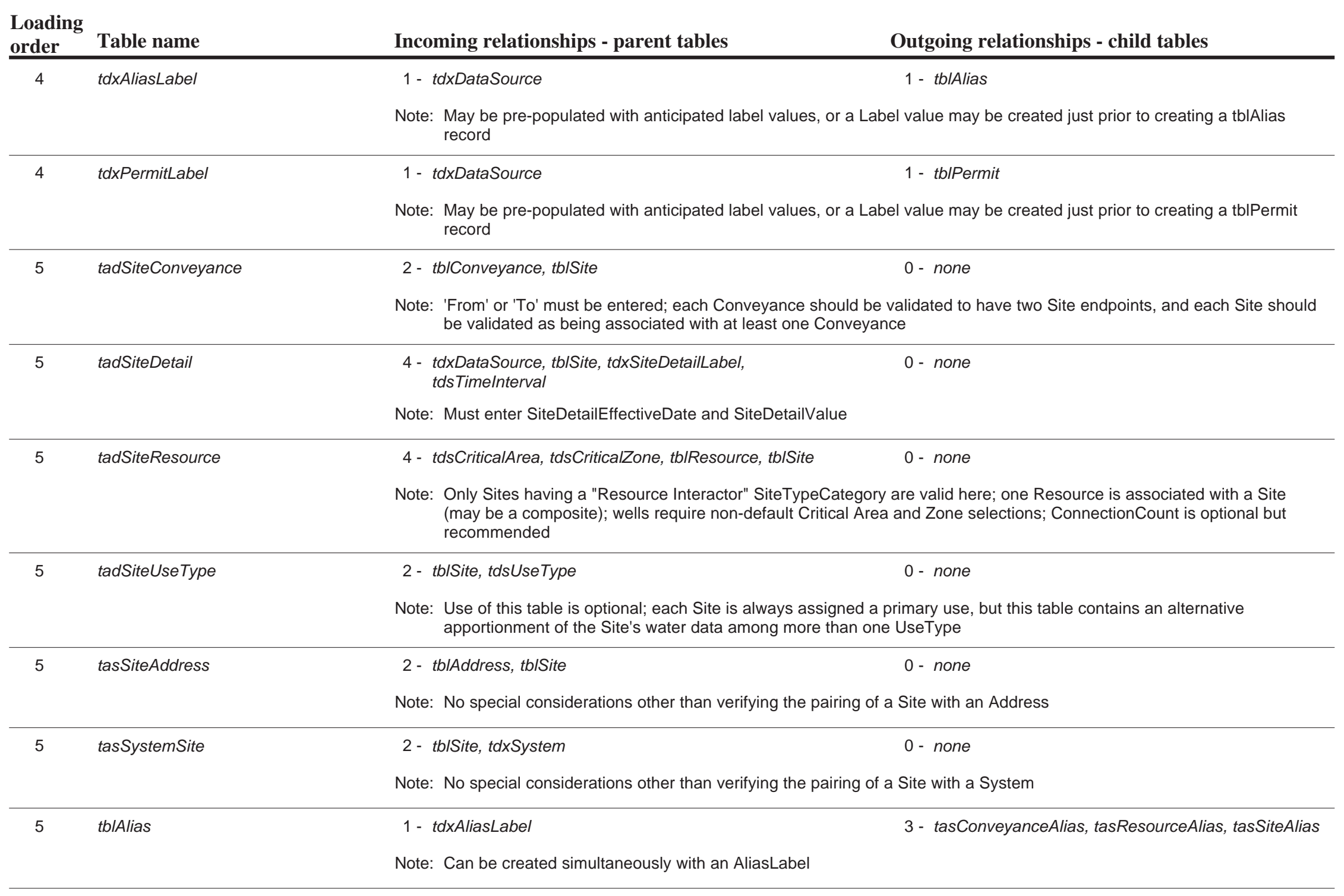


Table 1. Table loading order and related information for the New Jersey Water-Transfer Data System (NJWaTr)—Continued

\begin{tabular}{|c|c|c|c|}
\hline $\begin{array}{l}\text { Loading } \\
\text { order }\end{array}$ & Table name & Incoming relationships - parent tables & Outgoing relationships - child tables \\
\hline \multirow[t]{2}{*}{5} & tblPermit & 2 - tdxPermitLabel, tdsPermitSeries & 2 - tasOwnerPermit, tasSitePermit \\
\hline & & \multicolumn{2}{|c|}{ Note: Site or Owner must be known before creating a Permit association } \\
\hline \multirow[t]{2}{*}{5} & tblVolume & $\begin{array}{l}5-t d x \text { DataSource, } t d x \text { Staff, tblTransfer, } \\
\text { tdxVolumeMethod, tdxVolumeUnit }\end{array}$ & 1 - tadVolumeDetail \\
\hline & & \multicolumn{2}{|c|}{ Note: Volume information can be entered simultaneously with its tblTransfer record } \\
\hline \multirow[t]{2}{*}{6} & tadVolumeDetail & 2 - tblVolume, tdxVolumeDetailLabel & 0 - none \\
\hline & & Note: VolumeDetail must be entered & \\
\hline \multirow[t]{2}{*}{6} & tasConveyanceAlias & 2 - tblAlias, tblConveyance & 0 - none \\
\hline & & \multicolumn{2}{|c|}{ Note: No special considerations other than verifying the pairing of a Conveyance with an Alias } \\
\hline \multirow[t]{2}{*}{6} & tasOwnerPermit & 2 - tblOwner, tblPermit & 0 - none \\
\hline & & \multicolumn{2}{|c|}{ Note: No special considerations other than verifying the pairing of an Owner with a Permit } \\
\hline \multirow[t]{2}{*}{6} & tasResourceAlias & 2 - tblAlias, tblResource & 0 - none \\
\hline & & \multicolumn{2}{|c|}{ Note: No special considerations other than verifying the pairing of a Resource with an Alias } \\
\hline \multirow[t]{2}{*}{6} & tasSiteAlias & 2 - tblAlias, tb/Site & 0 - none \\
\hline & & \multicolumn{2}{|c|}{ Note: No special considerations other than verifying the pairing of a Site with an Alias } \\
\hline \multirow[t]{2}{*}{6} & tasSitePermit & 2 - tblPermit, tblSite & 0 - none \\
\hline & & \multicolumn{2}{|c|}{ Note: No special considerations other than verifying the pairing of a Site with a Permit } \\
\hline
\end{tabular}


The first query, qrmVolumeUnitConversionFactor, creates the phrase and conversion factor from fields provided for that purpose in the two component tables. The phrase is constructed by combining unit terms from the fields DecimalUnit and QuantityUnit. The conversion factor is calculated as the product of the ConversionToMillion and ConversionToGallon fields of the component tables. The SQL statement for this query is

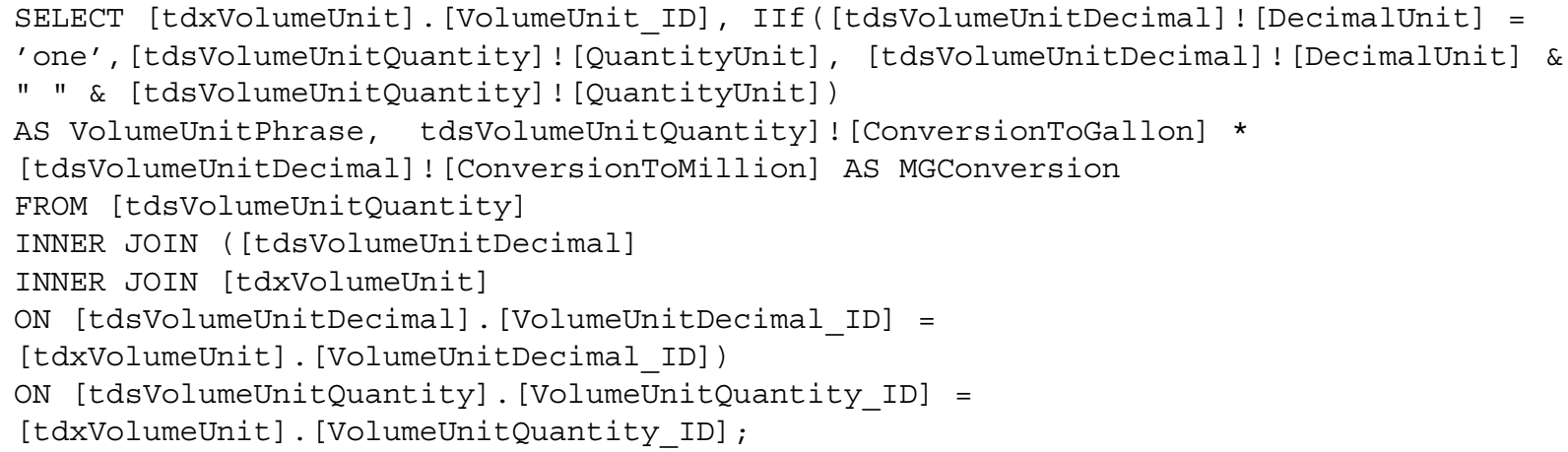

Note the IIf statement near the beginning of the query. When the decimal component of the constructed unit is "one," no explicit statement of that is desirable in the actual unit phrase itself. The IIf constraint in the above query presents the volume term when the decimal term is "one;" otherwise, it presents the decimal term, a space, and the volume term. Not using the word "one" avoids creating an awkward phrase for the unit; for example, the phrase value "cubic feet" is the desired phrase compared with "one cubic feet."

The $t d x$ VolumeUnit table is updated by running the query, qrmVolumeUnitUpdateNEW. It identifies records where MGConversion = "-1" and applies the above query only to those records that need the update in the VolumeUnitPhrase and MGConversion fields. The SQL statement for this query is

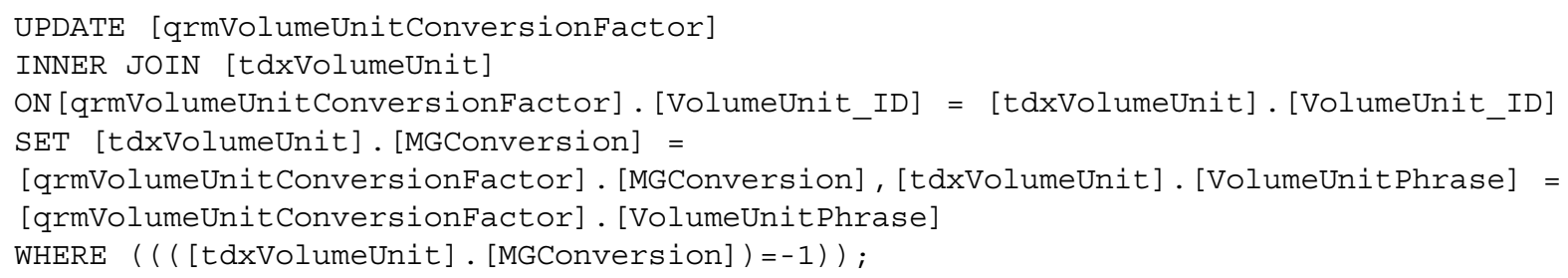

A variant on the second query is provided in NJWaTr to update all the VolumeUnit phrases and conversion factors in the $t d x$ VolumeUnit table. This is used whenever conversion factors need to be corrected from changes made to the component tables. The query qrmVolumeUnitUpdateALL is identical to the query immediately above, except that it lacks the constraint of updating $t d x$ VolumeUnit records only where MGConversion = "-1" (the SQL WHERE clause is not used).

\section{Volume Conversion to Common Units and Updating Transfers}

Original Volume data for a Transfer are entered into the tblVolume table and consist of a value and the units for that value as originally received or created using a specified method and data source. The units are identified in NJWaTr by a selection from the $t d x$ VolumeUnit table. The default Volume value for each Transfer must be converted to common units (MG, million gallons) and entered into the tblTransfer table in the field VolumeMG. Initially, the VolumeMG value for newly created Transfers is assigned a default value of "-1", and this is used as a flag for values that have not yet been updated. Replacing the "-1" flag 
with a valid, converted Volume value is done by using two queries. One query sets things up and the other performs the update. Only the second query must be run.

The first query is qrmTransferConvertDefaultVolumes; it identifies the default Volume entries in tblVolume for each record in tblTransfer and calculates the equivalent converted Volume in MG common units based on the original VolumeUnit. The actual calculation is RawVolumeValue* MGConversion. This query is not run but only provides the set of records that represents converted default rates from tblVolume to the next query. The SQL statement for this query is

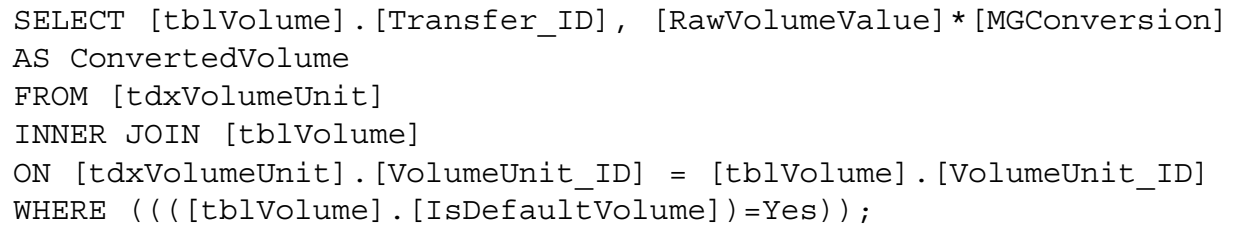

The second query, qrmTransferUpdateNEW, is run to perform the update. This query links tblTransfer records where VolumeMG $=$ "-1" to the first query's set of converted Volume values and replaces the default "-1" VolumeMG values in tblTransfer. The SQL statement for this query is

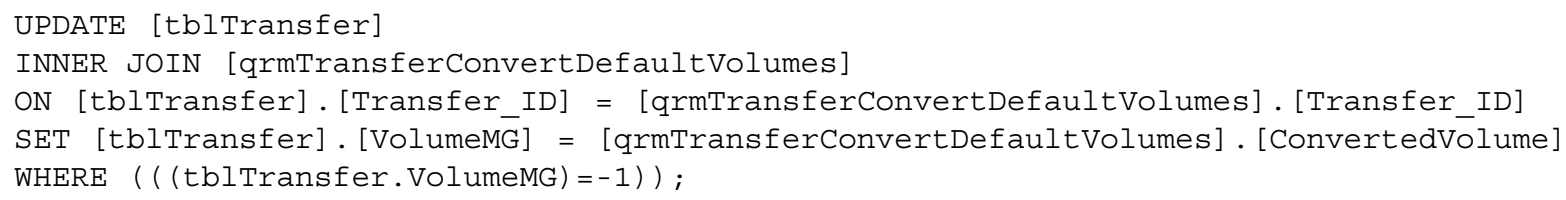

A variant on the second query is provided in NJWaTr to update all the VolumeMG values in the tblTransfer table. This is to be used whenever a conversion factor for one or more records in tdxVolumeUnit is changed or when adjustments might be made to RawVolumeValues in the tblVolume table. The query qrmTransferUpdateALL is identical to the query immediately above, except that it lacks the constraint of updating Transfer records only where VolumeMG = "-1" (the SQL WHERE clause is not used).

\section{Conveyance Action Phrase Updates}

The domain table $t d s$ ConveyanceAction provides a list of Site-type functions performed on a Conveyance and has a phrase field (ConveyanceActionPhrase) to describe that action (for example, the phrase "From ground-water withdrawal To potable-water treatment plant"). The phrase is constructed from the same terms used in the tdsSite Type table to describe the Sites at the To and From ends of the Conveyance. Exact correspondence between terms used to describe Sites and those used to describe Site connections (Conveyances) is desirable. Although the tdsConveyanceAction domain table is considered complete (static), the ability to update the phrase field will accommodate any potential changes to terms used in the tdsSite Type table. In the event that additions are made to the $t d s$ ConveyanceAction domain, the same update facility will create the correct ConveyanceActionPhrase.

The tdsConveyanceAction table contains two pseudo-key fields, SiteTypeFromID and SiteTypeToID. These fields contain values that correspond to SiteType_ID values in tdsSiteType. The purpose of the pseudo-keys is to allow transient linkages to be made between the tdsSiteType table and the tdsConveyanceAction table to automatically update the phrase field. Updating the field ConveyanceActionPhrase requires four queries - three to set things up and one to perform the update. Only the last query must be run. 
Because there is a one-to-two relationship between a ConveyanceAction and its two SiteType ends, two queries are needed to separately establish SiteType terms for the From and To ends of the Conveyance. A link is made between the tdsConveyanceAction and tdsSiteType tables, and type terms are collected separately with the text "From" and "To" added. The names and SQL statements for these two queries follow.

\section{qrmConvActionSiteTypeFrom}

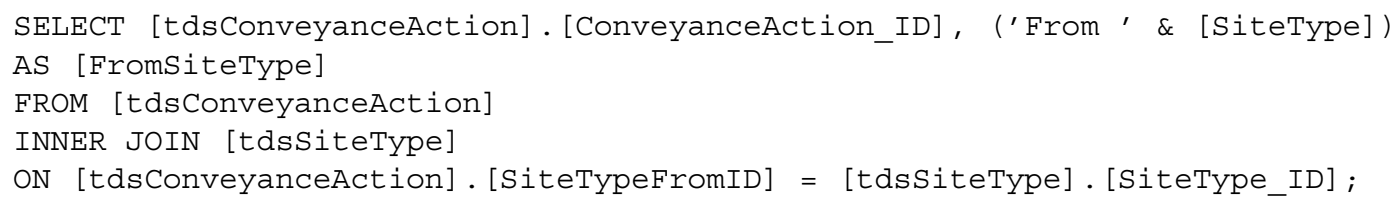

\section{qrmConvActionSiteTypeTo}

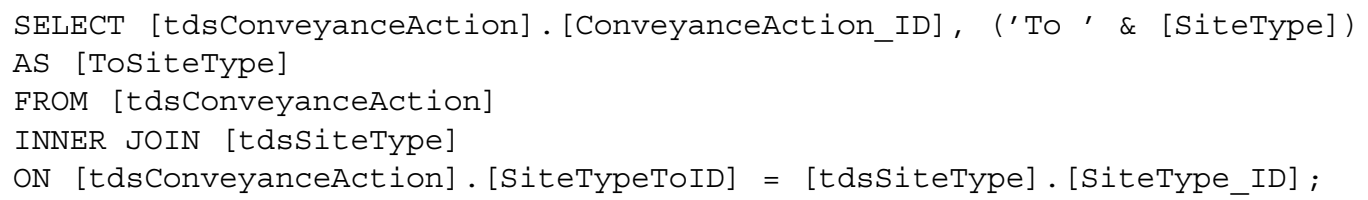

Next, a query is used to assemble the two parts into a phrase that is based on their common ConveyanceAction_ID values. The query and SQL statement that accomplish this are

\section{qrmConvActionPhrase}

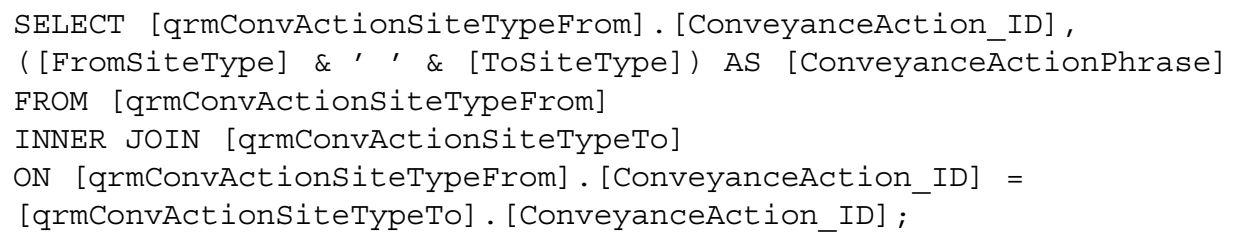

The last query applies the results of the previous query to perform the update of the field ConveyanceActionPhrase in table $t d s$ ConveyanceAction. This is the only query that is actually run; the others provide the partial solutions to the update procedure. The query and SQL statement for this procedure are

\section{qrmConvActionPhraseUpdate}

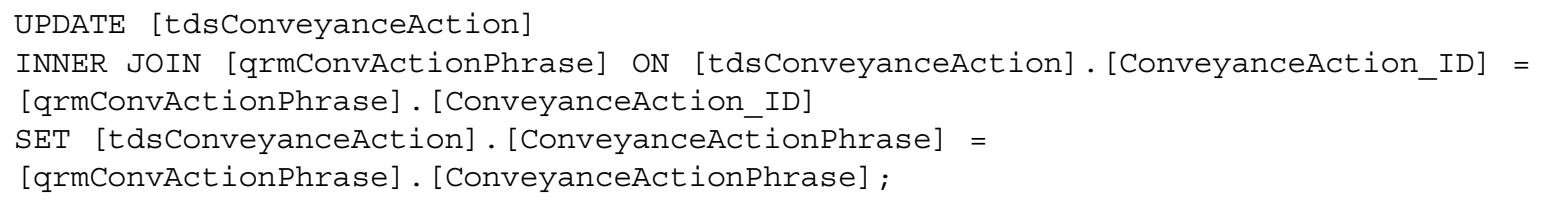

\section{Construction and Use of Views}

The number of individual NJWaTr tables and the complexity of relationships between them have the potential to be confusing to users. Although the primary reason for most of the individual tables is to respect the rules of normalization and, thus, provide significant integrity and list-maintenance advantages for the data, the task of manually collecting the correct sets of tables in a query to perform routine exploratory tasks should not be an impediment to using the database. Most of the sets of related tables in NJWaTr subject areas have been pre-assembled into single objects for further manipulation. These general-purpose queries are called "Views" and are essentially virtual tables; they do not exist as physical file structures but rather 
as named SQL statements stored in the database. When run or used in forms or reports, Views create the appearance of, and act like, a physical table.

A typical View is a query that begins with a base table, then gathers attributes (fields) from related tables and drops the keys that joined them. Thus, fields from related domain and data tables are added to those of the View base table. Views help users see the data in a familiar flatfile or spreadsheet format for browsing and checking data without the visual distraction of keys and relationships. Views can be combined with other Views to create elaborate View assemblies. In addition, Views can be used to export a physical flatfile directly to file types that can be imported by other applications.

Views are stored as queries with a qrv prefix in NJWaTr. In most cases, a View is a complete assembly of nearest-neighbor-related domain tables centered on a single subject area. After a View is created, it then can be combined with others to form much larger data objects that can be handled, and whose data can be evaluated, at once. In some Views, there is an abbreviated assembly of items due to one-to-many relationships between tables that compose the View. An example of the latter is the ignoring of non-default Volume values in assembling a View of Transfers. (Multiple Volume estimates can be stored for a single Transfer, whereas only the default is presented in the View.) Likewise, each Location's primary state, county, MCD, and HUC are associated with the qrvLocation View, instead of creating many new columns for those instances where more than one item within each spatial domain relates to a single Location. These compromises might affect an analysis and need to be recognized; users are encouraged to create their own optimized Views for specific needs.

\section{Example of View Construction}

An example of View construction is provided to illustrate the method and result common to all standard NJWaTr Views. The Location subject area in NJWaTr consists of 14 tables (fig. 10). Four of those are association tables for storing multiple states, counties, MCDs, and HUCs for a Location, and for each Location only one of each is designated as the primary. Five queries are needed to create a View of the Location subject area-four to identify the primary selections from the spatial domains (if present) for each Location (qrvLocationPrimaryState, qrvLocationPrimaryCounty, qrvLocationPrimaryMCD, and qrvLocationPrimaryHUC), and one to incorporate those queries and gather other fields from domain tables with the base tblLocation table fields (qrvLocation). Note that the MCD and HUC domains have their own higher level domains, so the gathering of primary MCDs includes the Drought Region in which they occur, and the primary HUCs include the related Water Management Area and larger Water Region.

The three tables and four subqueries involved in the Location View are shown in figure 19 as they would be seen in the upper panel of the MS Access query Design view. Note the arrows on the relationship lines that link fields in the base table to the same field in the subqueries and domains; these indicate that a LEFT JOIN has been established in SQL to ensure that the query returns all records from tblLocation, even when no primary entries are present in the subqueries for a particular Location_ID value.

The qrvLocation View is built to collect all non-key fields from all joined tables and can use the Location_ID to serve as the key for the entire assembly. The result is a single virtual table where each record describes a Location by using 39 data fields (plus the LocationMemo field) anchored to the Location_ID key. The appearance of the qrvLocation View when selected for use in other queries also is shown in figure 19. This virtual table can be used in other queries, manipulated in forms and reports, or exported to spreadsheets or text files without further regard for the complex of 14 original tables and 4 preliminary queries that formed it. 


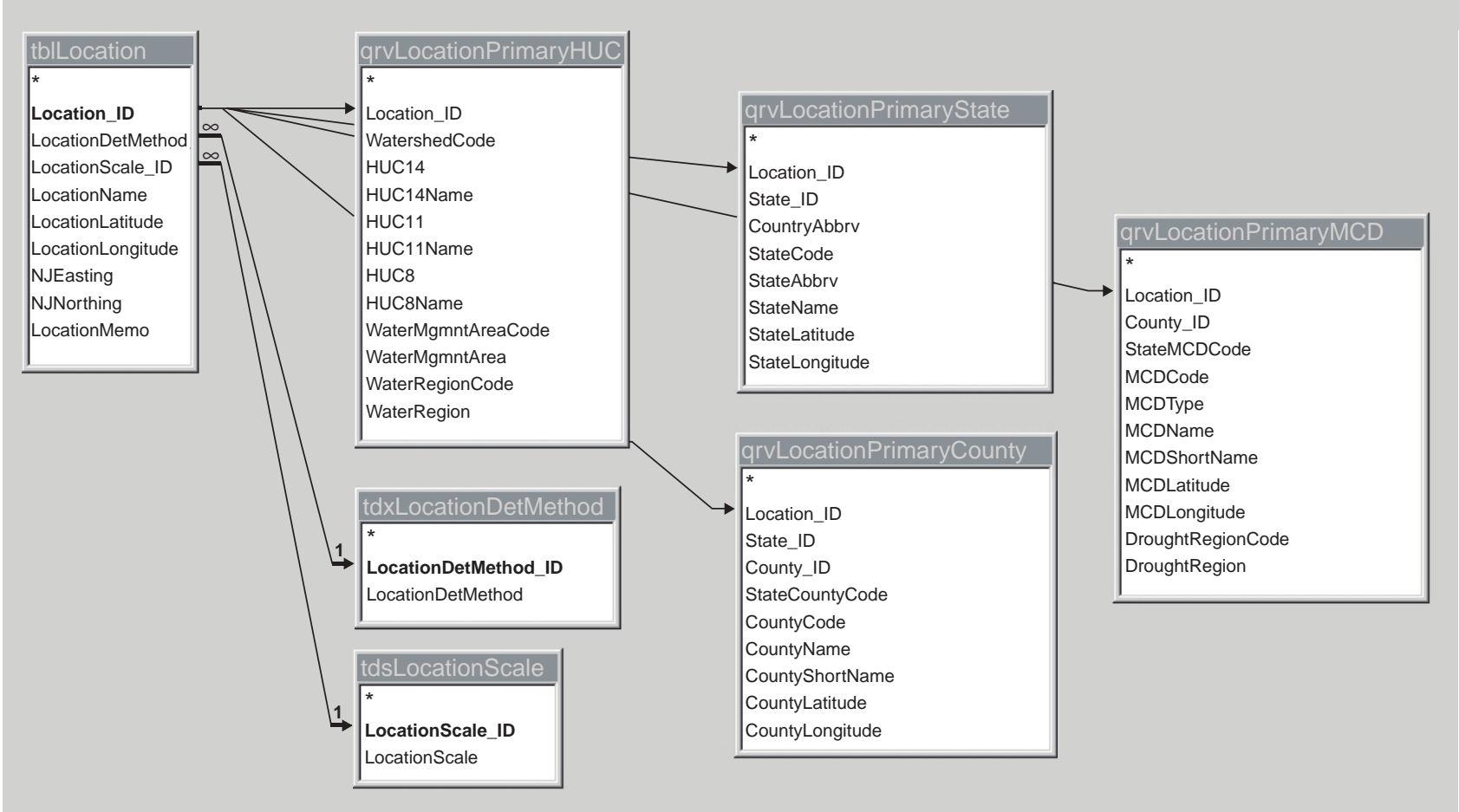

\begin{tabular}{|l|}
\hline GrvLocation \\
\hline * \\
\hline Location_ID \\
\hline LocationDetMethod \\
LocationScale \\
LocationName \\
LocationLatitude \\
LocationLongitude \\
NJEasting \\
NJNorthing \\
CountryAbbrv \\
StateCode \\
StateAbbrv \\
PrimaryState \\
StateLatitude \\
StateLongitude \\
StateCountyCode \\
CountyCode \\
PrimaryCounty \\
CountyShortName \\
CountyLatitude \\
CountyLongitude \\
StateMCDCode \\
MCDCode \\
MCDType \\
PrimaryMCD \\
MCDShortName \\
MCDLatitude \\
MCDLongitude \\
DroughtRegionCode \\
DroughtRegion \\
WatershedCode \\
PrimaryHUC14 \\
HUC14Name \\
HUC11 \\
HUC11Name \\
HUC8 \\
HUC8Name \\
WaterMgmntAreaCode \\
\hline
\end{tabular}

Figure 19. A View assembly (left) and a virtual table (right, not all fields shown) focused on the tb/Location table. 
Another type of View is created using a crosstab query in MS Access. Crosstab queries, also known as pivot queries, create a table View with new columns based on values in a field and are very useful for denormalizing or rotating data stored in or through association tables. For example, Owner Permits are assigned by pairing an Owner_ID with a Permit_ID in tasOwnerPermit and refer to an entry in tblPermit. To determine all the Permits associated with Owners, one can create a crosstab query of tblPermit and related tables and use the PermitLabel as the source of the new column labels. The result is a virtual table with Owner_ID as the key and a column for each PermitLabel associated with Owners. The query layout in MS Access and output for some test data are illustrated in figure 20; each of the four PermitLabels that were associated with Owner Permits are presented as columns anchored to individual Owner_IDs. This View can be linked directly to the tblOwner table through the Owner_ID to provide the Permits as extended Owner attributes. The column name could be made more informative by concatenating the PermitLabel and the DataSource descriptor values.

Views can be linked to other Views to create large customized flatfile objects for export, for use in forms and reports, or for any other purpose. An example of how such a complex object would be assembled is shown in figure 21; Views of Site, Resource, Owner, and Location are combined to provide a selected subset of 37 Site descriptors from 29 original tables (4 basic data, 19 domain, and 6 association) anchored to a single Site_ID key. The virtual table resulting from the View and a form that uses most of the fields is shown in figure 22. The form demonstrates an exploratory tool for the NJWaTr data model. In MS Access, drill-down filtering of data is possible directly within the form, and the select subset of Sites gathered on the basis of multiple-field filter criteria can be saved for later reference with a query created from the form. Custom Views can be constructed and combined with forms to create specialized exploratory tools.

\section{Standardized and Optimized Views}

NJWaTr includes 27 pre-assembled standard Views. These are listed along with their base PK and a brief description in table 2. These Views are assembled by gathering fields focused on each major subject area in the database. The four preliminary Location Views that gather only the primary selections from the spatial domains are included, along with a View that reveals the parent/child structure of composite Resources. Views that are based on association tables can be modified easily for crosstabulation, as described previously, to create denormalized structures for Address, Permit, Alias, and User-Defined Detail tables, thereby creating new virtual fields associated with subject area PKs. The standard Views will produce equivalent output from any independent implementation of NJWaTr (different data, but comparable views).

The standard Views can be used as templates for creating custom optimized queries and Views. The standard View contains all value fields from nearby tables, and the underlying query might perform sluggishly on large data files. A custom query can drop unnecessary tables, fields, and relationships from a standard View, and this economy will significantly improve performance. Custom queries that start with a View template can rearrange or rename fields and impose conditional criteria, thereby creating precise subsets of data in custom formats. An optimal View would include only those tables containing the query output fields and any other tables needed to create a relational pathway between those tables. Many of the standard Views use INNER, LEFT, and RIGHT JOINs in the SQL statement, so the user is cautioned to examine a query structure carefully before modifying it for another use. 


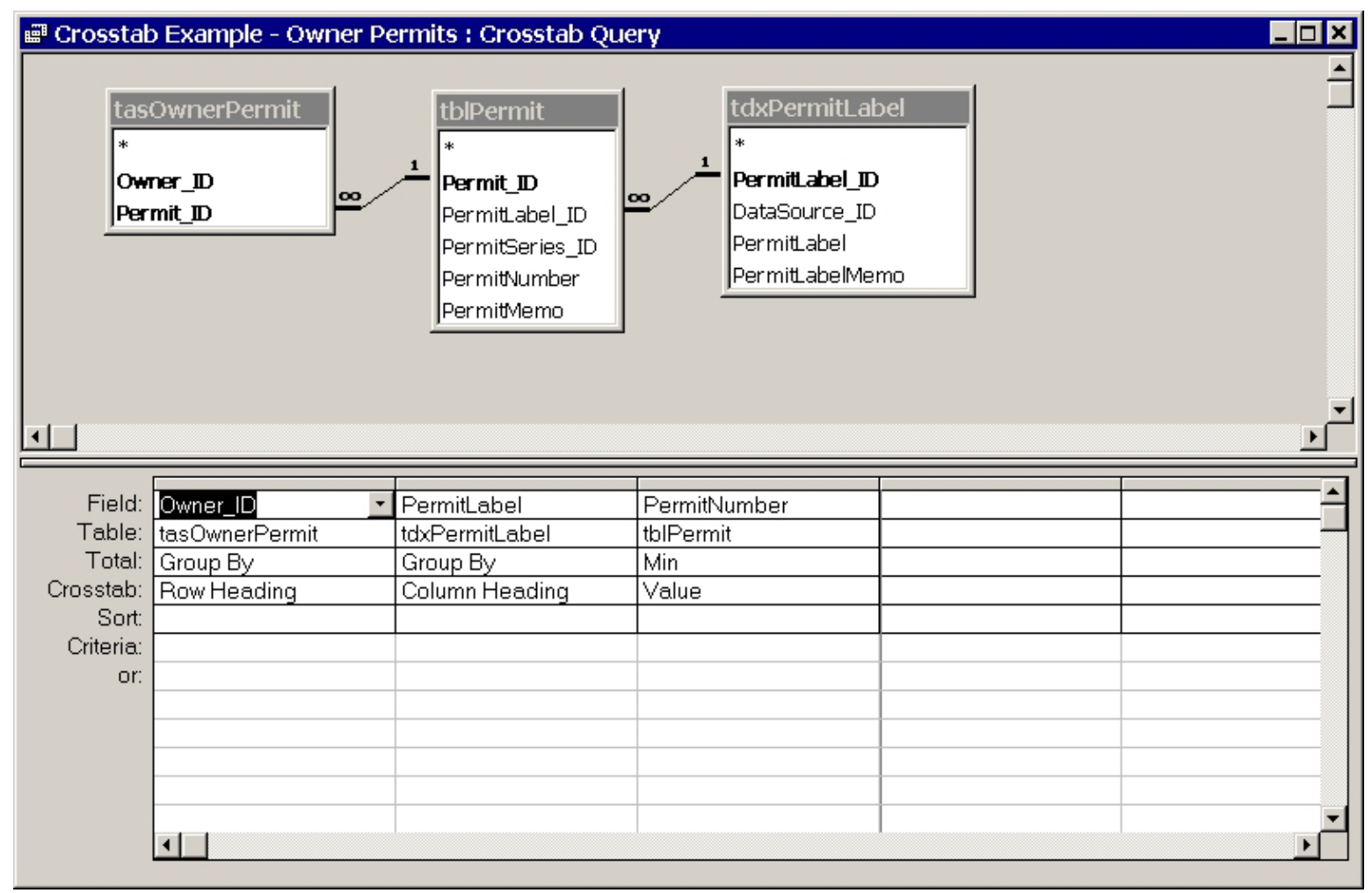

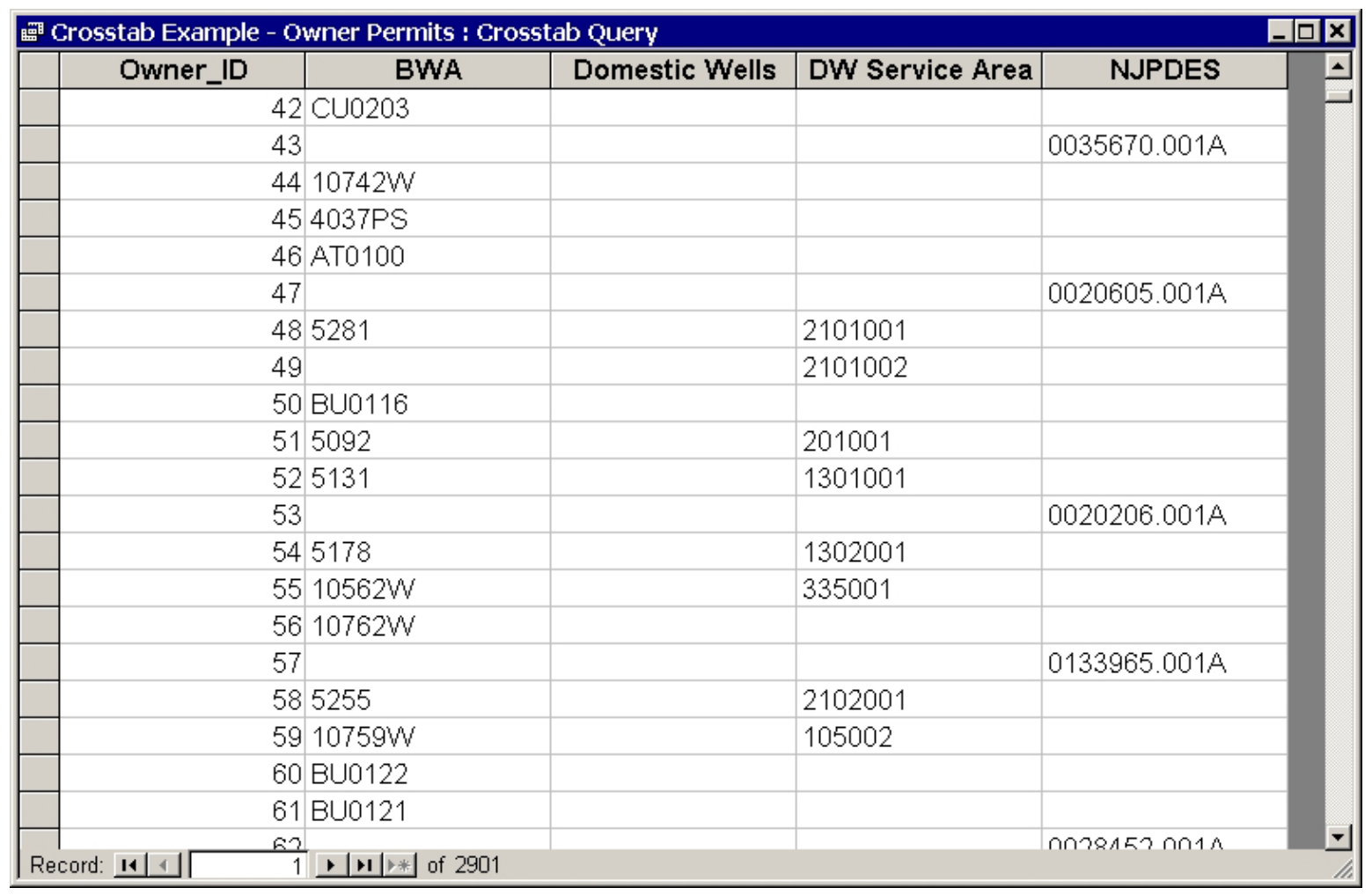

Figure 20. A crosstab-query design (top) and a crosstab-query output (bottom). 


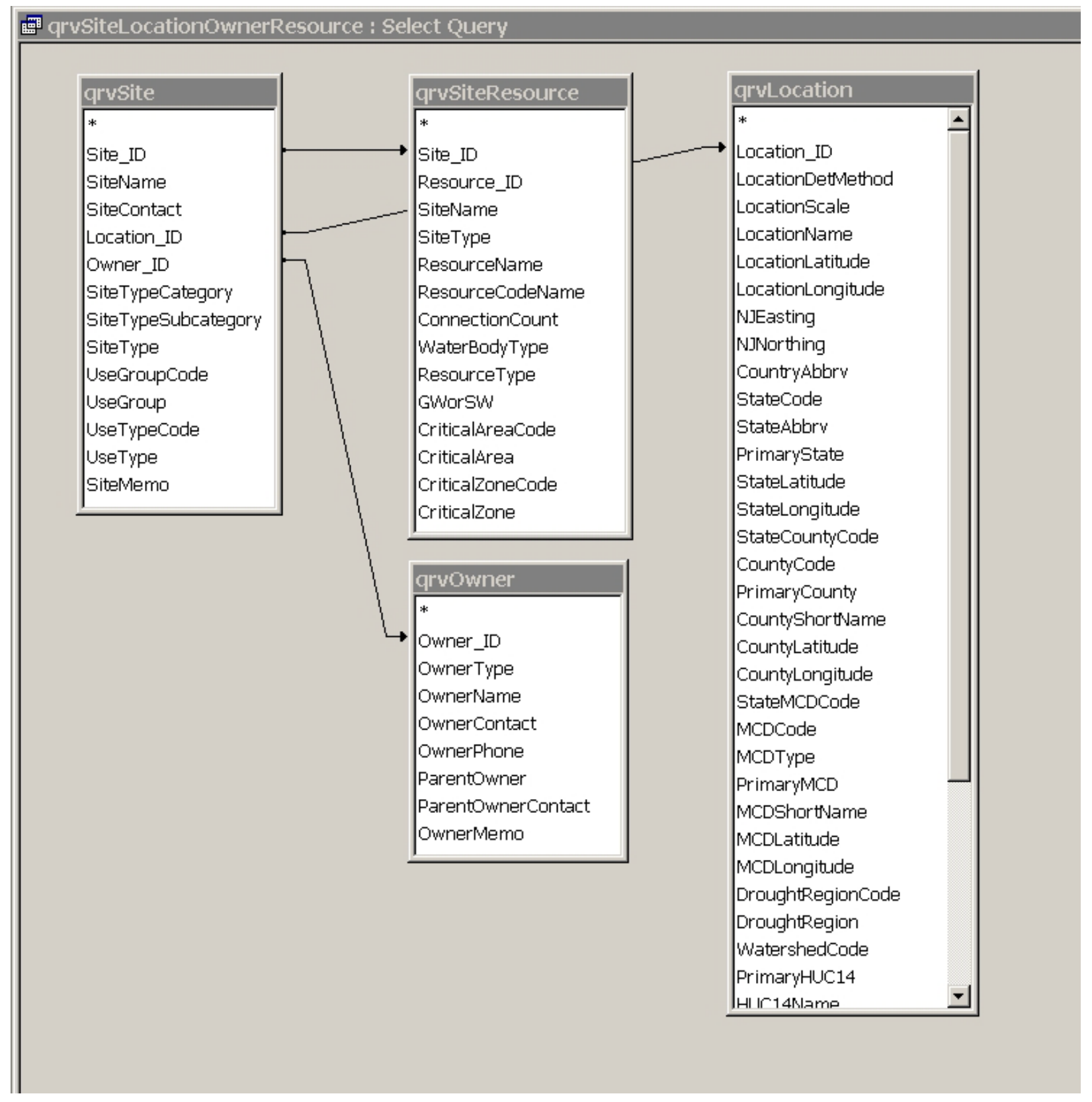

Figure 21. A complex View assembly made up of other Views. 


\begin{tabular}{l} 
qrvSiteInformation \\
\hline * \\
Site_ID \\
SiteName \\
SiteTypeCategory \\
SiteTypeSubcategory \\
SiteType \\
SiteContact \\
SiteMemo \\
UseGroupCode \\
UseGroup \\
UseTypeCode \\
UseType \\
OwnerName \\
OwnerType \\
Parentowner \\
OwnerContact \\
OwnerPhone \\
LocationName \\
LocationScale \\
LocationDetMethod \\
LocationLatitude \\
LocationLongitude \\
NJNorthing \\
StateAbbry \\
CountyShortName \\
MCDShortName \\
PrimaryHUC14 \\
HUC14Name \\
WatershedCode \\
WaterRegionCode \\
WaterRegion \\
WaterMgmntAreaCode \\
WaterMgmntarea \\
DroughtRegion \\
ResourceName \\
WaterBodyType \\
CriticalArea \\
CriticalZone \\
\hline
\end{tabular}

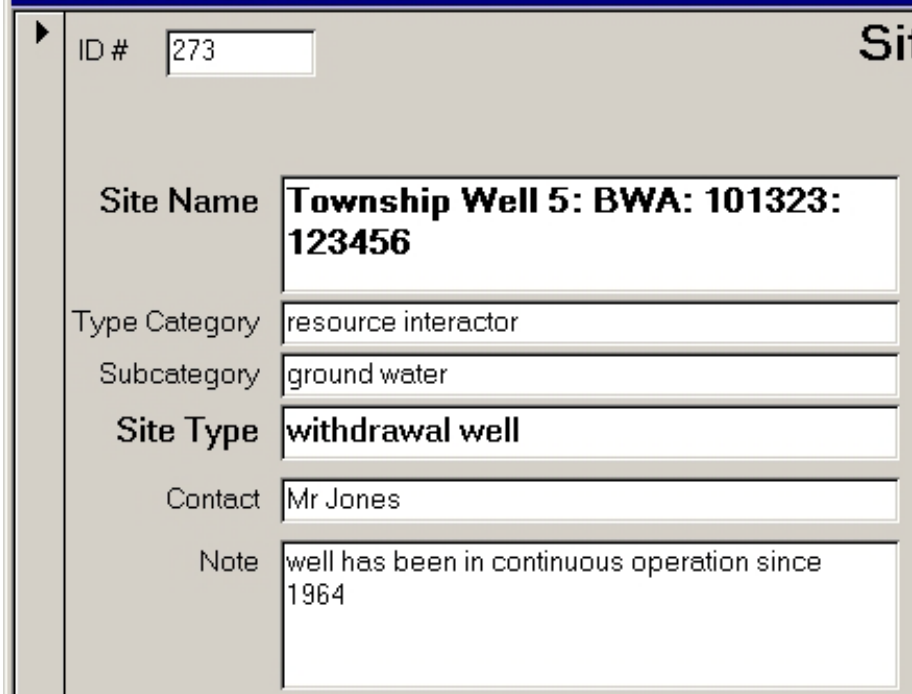

\section{Water-use Classification}

Group 5 potable supply

Type $T$ institutional

\section{Owner Information}

Name Anywhere Twp Bd of Education

Type Municipal

Parent Owner

Contact Ms Smith

Phone (609) $555-1234 \times 321$
Site Information

\section{Location Information}

Name Ficticious Place Code 101323:123456

Scale Point

Method GPS field

\begin{tabular}{|c|c|c|}
\hline Lat/Lon & 390000 & 750000 \\
\hline NJ East/Nort & 340000.123 & 280000.123 \\
\hline
\end{tabular}

Town/City Franklin

State/County NJ Gloucester

HUC 14 $\longdiv { 0 2 0 4 0 2 0 6 1 3 0 }$ Indian Branch (Scotland

NJ SWID 17 RAO3

Water Region 5 Lower Delaware

Water Mgmnt Area 17 Maruice, Salem, and Cohansey

Drought Region Coastal, South

\section{Water Resource}

Resource Name Tch

Water Body Type Aquifer-freshwater

Critical Area Critical Area designated in the Camden area of New Jersey

Critical Zone Well is within the composited area of a critical area but does not tap a regulated aquifer

Figure 22. A virtual table made from a complex View assembly (left) and a form based on the virtual table (right). 
Table 2. New Jersey Water-Transfer Data System (NJWaTr) standard preassembled views

[MCD is a Minor Civil Division (town or other subcounty geographic region) of the U.S. Census Bureau; HUC is a Hydrologic Unit Code of the U.S. Geological Survey]

\begin{tabular}{|c|c|c|}
\hline View (query) name & View primary key & View description \\
\hline qrvConveyance & Conveyance_ID & Joins conveyance action and type domains to conveyances \\
\hline qrvConveyanceAlias & Conveyance_ID & $\begin{array}{l}\text { Assembles alias data applying only to conveyances; may } \\
\text { be crosstabbed }\end{array}$ \\
\hline qrvConveyanceDetail & Conveyance_ID & $\begin{array}{l}\text { Joins detail label domain to conveyance details; may be } \\
\text { crosstabbed }\end{array}$ \\
\hline qrvConveyanceOwner & Conveyance_ID & $\begin{array}{l}\text { Assembles owner data applying only to conveyances; also } \\
\text { has Owner_ID }\end{array}$ \\
\hline qrvLocation & Location_ID & $\begin{array}{l}\text { Joins scale and determination method domains, and } \\
\text { includes only primary state, county, MCD (town), HUC, } \\
\text { drought region, water region and management area }\end{array}$ \\
\hline qrvLocationPrimaryCounty & Location_ID & Assembles primary county information for a Location \\
\hline qrvLocationPrimaryHUC & Location_ID & Assembles primary HUC information for a Location \\
\hline qrvLocationPrimaryMCD & Location_ID & Assembles primary MCD information for a Location \\
\hline qrvLocationPrimaryState & Location_ID & Assembles primary state information for a Location \\
\hline qrvOwner & Owner_ID & $\begin{array}{l}\text { Joins owner type domain and parent-owner information to } \\
\text { owners }\end{array}$ \\
\hline qrvOwnerAddress & Owner_ID & Assembles address information applying only to owners \\
\hline qrvOwnerPermit & Owner_ID & $\begin{array}{l}\text { Assembles permit information applying only to owners; } \\
\text { may be crosstabbed }\end{array}$ \\
\hline qrvResource & Resource_ID & $\begin{array}{l}\text { Joins resource type and water body type domains to } \\
\text { Resources }\end{array}$ \\
\hline qrvResourceAlias & Resource_ID & $\begin{array}{l}\text { Assembles alias data applying only to resources; may be } \\
\text { crosstabbed }\end{array}$ \\
\hline qrvResourceDetail & Resource_ID & $\begin{array}{l}\text { Joins detail label and data source to resource details; may } \\
\text { be crosstabbed }\end{array}$ \\
\hline qrvResourceStructure & Resource_ID & $\begin{array}{l}\text { Assembles composite resources to show their child, } \\
\text { component resources }\end{array}$ \\
\hline qrvSite & Site_ID & $\begin{array}{l}\text { Joins site type and use type domains to sites; also has } \\
\text { Owner_ID and Location_ID }\end{array}$ \\
\hline qrvSiteAddress & Site_ID & Assembles address information applying only to sites \\
\hline qrvSiteAlias & Site_ID & $\begin{array}{l}\text { Assembles alias data applying only to sites; may be } \\
\text { crosstabbed }\end{array}$ \\
\hline qrvSiteDetail & Site_ID & $\begin{array}{l}\text { Joins detail label and category, time interval, and data } \\
\text { source to site details; may be crosstabbed }\end{array}$ \\
\hline qrvSiteLocationOwnerResource & Site_ID & $\begin{array}{l}\text { A comprehensive view of sites constructed from four } \\
\text { separate views }\end{array}$ \\
\hline qrvSitePermit & Site_ID & $\begin{array}{l}\text { Assembles permit information applying only to sites; may } \\
\text { be crosstabbed }\end{array}$ \\
\hline qrvSiteResource & Site_ID & Joins views of sites and resources; also has Resource_ID \\
\hline qrvSystem & System_ID & $\begin{array}{l}\text { Joins system type domain to systems; also has associated } \\
\text { Site_ID's }\end{array}$ \\
\hline qrvTransfer & Transfer_ID & $\begin{array}{l}\text { Joins time interval domain and volume view (default } \\
\text { values only) to transfers; also has Conveyance_ID }\end{array}$ \\
\hline qrvVolume & Volume_ID & $\begin{array}{l}\text { Joins method, staff, unit, and data source domains to } \\
\text { volumes; also has Transfer_ID }\end{array}$ \\
\hline qrvVolumeDetail & Volume_ID & Joins detail label domain to volumes; may be crosstabbed \\
\hline
\end{tabular}




\section{CUSTOMIZING AND EXTENDING THE DATA ARCHITECTURE}

NJWaTr was designed to be fully extendable and customizable for the specific needs of NJDEP or for individual projects. Two basic ways to extend the database to contain new data are to add fields or add tables. In either case, extensions to or customizations of the data model should respect existing conventions that apply to normalization, keys and relationships, domain table usage, and naming rules. It is recommended that the names of all custom accessory tables and fields be prefixed with the letter " $z$ " or other unique indicator to easily distinguish them from the fully defined, standard NJWaTr objects.

A field could be added to any table where its data can be expected to be readily available and regularly entered; however, fields should not be added to a working copy of the database to solve short-term needs, such as those that facilitate translating outside data into the NJWaTr format, or to accommodate an unusual data inquiry. Adding accessory tables to the structure is probably the easiest way to extend the data model and can provide a means to experiment with new data elements without affecting the data already stored. One-to-zero-or-one $(1: 0,1)$ relationships between existing and new accessory tables are the equivalent of adding fields to a table, but those fields are physically stored in a different place. Accessory tables have the added advantages of allowing the new data to be entered only for those records in the parent table that need them and protecting the current data by not altering existing structures. Specialized accessory tables that provide descriptive fields for only some members of an existing table are usually referred to as subtype tables (Fleming and von Halle, 1989, p. 90). For example, the association table tadSiteResource is a subtype table; it contains Site_IDs of only resource-interactor Sites to pair with a Resource and related domains. As an additional example, to store information specific to only those Sites that are drilled wells, a subtype table named $z \mathrm{tblWell} \mathrm{could} \mathrm{be} \mathrm{created} \mathrm{to} \mathrm{contain} \mathrm{fields} \mathrm{for} \mathrm{construction} \mathrm{date,} \mathrm{total} \mathrm{depth,} \mathrm{depth} \mathrm{to} \mathrm{open} \mathrm{interval,}$ and other details that do not apply to Sites in general. The PK of the ztblWell table would be Site_ID implemented as a FK from the tblSite table, and the only entries in ztblWell would be for Sites (using their Site_ID) that are drilled wells and for which the user wants to store the extra information. (Note that such information also could be stored in the tadSiteDetail table by creating the appropriate SiteDetailLabel values.)

It is expected that some domain entities may better serve database users if they are extended into more complex domain structures through nesting or clustering. An indication that domain reevaluation is needed would be when an apparent exception presents difficulty in selecting from a domain for particular records in the child table or when a domain provides too much detail for grouping and sorting purposes (a higher category domain is needed).

In addition, the data model can be simplified by dropping fields from the standard database because they are not being used. That decision should be based on the value of the data to be stored, not on the difficulty of obtaining or entering it. Data elements that are discovered to be ambiguous will require reevaluation to determine if they should be stored at all and whether they are being modeled and stored in the correct way. Absence of data for a required field can always be accommodated with a value that represents an absence condition, and nearly all of the domain tables in NJWaTr are equipped with a record for such use.

By linking to NJWaTr from a separate MS Access database, accessory tables can reside outside of NJWaTr and still be able to use its data. The database containing the linked tables is often called a front-end database, whereas the database containing the actual tables and data would then be the back-end. Linking to NJWaTr would be the preferred method for preparing original import data for inclusion into the NJWaTr tables, where a staging area front-end database is created for the import tables and original data manipulations, and new records are passed to the linked NJWaTr tables only after they have been properly qualityassured, formatted, and provided with necessary key values. A staging database as described can be emptied and reused as needed to process new imports. 
A front-end database is also a good place to create linkages to other databases. Links to NJWaTr tables would be present along with links to another database. One or more fields that contain identity information are required to connect the data, and this could be done in a query without altering either source database. New association tables also could be created in the front-end to pair corresponding items by their key fields or other identifiers. For example, water-quality data could be accessible to NJWaTr if a waterquality database used the same SiteName attribute.

\section{SUMMARY}

Changing demographics and economic factors continue to place additional demands on New Jersey's finite water resources. Domestic and industrial needs typically are balanced with agricultural, recreational, wastewater discharge, and ecological uses. These finite water resources can be particularly stressed during periods of drought. A key component in managing the State's water resources is determining water availability.

To determine the amount of water that may be available in a watershed, one must understand the water dynamics - the amount of rainfall, the amount and fate of surface- and ground-water withdrawals, the amount and location of surface- and ground-water discharges, the depletive and consumptive uses of water, the amount and location of water transfers between watersheds, and the amount of water that is naturally leaving the watershed as streamflow and evapotranspiration. An accounting or water-budget model is needed to establish how much ground and surface water might be available in a watershed at different times and under different use scenarios. One problem encountered in doing such analyses is the availability of tools needed to store and manipulate the water withdrawal, use, and discharge data collected by the State; therefore, a database design was developed as a tool to aid in the analyses.

The New Jersey Water-Transfer Data System (NJWaTr), developed in cooperation with the New Jersey Department of Environmental Protection, is a database design for the storage and retrieval of wateruse data and is based on a core model developed for the New England region. NJWaTr can handle data on many facets of water use, including (1) various types of water-use activities (withdrawals, returns, transfers, distributions, consumptive use, wastewater collection, and treatment); (2) descriptions, classifications, and locations of places and organizations involved in water-use activities; (3) details of measured or estimated volumes of water associated with water-use activities; and (4) information on data sources and water resources associated with water use. NJWaTr can be used for large projects (states and regions) and small, focused projects (watershed studies).

The core NJWaTr model pairs Sites to form unidirectional Conveyances, and time-bounded water Transfers are stored for the Conveyances. The Conveyance-based model facilitates a water-network approach to water-use data storage, investigation, and visualization. Information also is stored about Locations, Owners, water Resources, multi-Site Systems, Addresses, DataSources, Permits, Aliases, and UserDefined Details.

Standards for normalization, keys and relationships, indexes, and naming were applied to the data model and its physical implementation as a stand-alone MS Access database. The database consists of 76 tables, 311 fields, and 95 defined relationships. Five functional types of tables are recognized in NJWaTr and described. Domain tables constitute more than half the tables and fields in NJWaTr. The domains are pre-populated with classification and descriptive terms and serve as flexible tools for grouping, sorting, filtering, and summarizing information. Operational considerations include index management, table loading order, and use of maintenance update queries and Views. NJWaTr can be customized or extended by adding new fields and tables, by dropping existing fields, and by linking to other databases. The data stored in 
NJWaTr can be exported or linked to other applications as needed, such as for supply monitoring, statistical analysis, and visualization using Geographic Information Systems (GIS).

NJWaTr was designed for storage and retrieval of water-transfer data for New Jersey. Domains can be amended for other areas and for different major water-use patterns. The data contained in a NJWaTr database can be transferred to other data-management tools. Careful management of the quality of data stored using the NJWaTr structure will facilitate the use of accurate, complex investigative queries to assist with management decisions about water use and water resources, and will help to avoid obsolescence of the data. 


\section{REFERENCES CITED}

Fleming, C.C., and von Halle, Barbara, 1989, Handbook of relational database design: Reading, Mass., Addison-Wesley Publishing Company, 605 p.

Hernandez, M.J., 1997, Database design for mere mortals-A hands-on guide to relational database design: Reading, Mass., Addison-Wesley Publishing Company, 480 p.

Hoffman, J.L., and S.E. Lieberman, 2000, New Jersey water withdrawals 1990-1996: New Jersey Geological Survey Open-File Report OFR 00-1, 37 p., 6 app.

Lonigro, Michael, 1997, The case for the surrogate key: Database Programming and Design, Online Extra, accessed September 6, 2002, on the World Wide Web at URL http://www.dbpd.com/vault/ 9805xtra.htm.

National Institute of Standards and Technology, 1993, Standard for integration definition for information modeling (IDEF1X): Federal Information Processing Standards Publication 184, 155 p., accessed September 6, 2002, on the World Wide Web at URL http://www.itl.nist.gov/fipspubs/0-toc.htm.

Roman, Steven, 1999, Access database design and programming, (2d ed.): Sebastopol, Calif., O'Reilly and Associates, $409 \mathrm{p}$.

Tessler, Steven, 2002, Data model and relational database design for the New England water-use data system (NEWUDS): U.S. Geological Survey Open-File Report 01-359, 70 p., 1 pl., 2 app., on 1 CDROM, accessed September 6, 2002, on the World Wide Web at URL http://water.usgs.gov/pubs/of/ ofr01359/.

Water Science and Technology Board, Committee on USGS Water Resources Research, 2002, Estimating water use in the United States: A new paradigm for the National Water-Use Information Program: Washington, DC, National Academy Press, 176 p., accessed September 6, 2002, on the World Wide Web at URL http://books.nap.edu/books/0309084830/html/. 
Appendix 1: Data Dictionary for the New Jersey Water-Transfer Data System (NJWaTr) 


\section{Appendix 1: Data Dictionary for the New Jersey Water-Transfer Data System (NJWaTr)}

How to read the Data Dictionary listings:

Each table starts on a new page. The top section shows the table information:

Table Name is self-explanatory.

Table Description describes the table contents or function.

Number of Fields in the table.

Number of Records at the time the dictionary was generated.

Dependency - Full means the table Primary Key (PK) contains a Foreign Key (FK) field; Partial means the table contains a required, non-PK, FK field; Independent means the table has no FK fields.

Loading Order - 1 if there are no incoming relationships, 2 if the table has at least one incoming relationship from a level 1 table, 3 if the table has at least one incoming relationship from a level 2 table, and so forth.

Recursive is checked if the table has a self-join, recursive Foreign Key field.

Below the table section, each field in the table is listed along with the definition information:

A number indicating the field position within the table.

Field Name is self-explanatory.

PK is checked if the field is part of the Primary Key for the table.

FK is checked if the field is a Foreign Key--pointing to data in a 'parent' table.

Rqd is checked if the field is required to have a value for each record in the table; if unchecked the field is optional.

Type indicates the data type of the field.

Size indicates the allowed number of characters for Text fields, "--" for Memo fields, or the number of bytes used for storage for other data types.

Default is the value automatically assigned to the field if no value is entered; it often refers to a foreign key value representing a selection from a domain table.

Description of the field. 
Appendix 1: Data Dictionary for the New Jersey Water-Transfer Data System (NJWaTr) — Continued

Table Name $\quad$ Page

tadConveyanceDetail ............................ 1-5

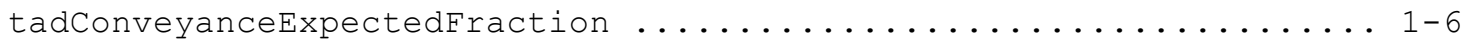

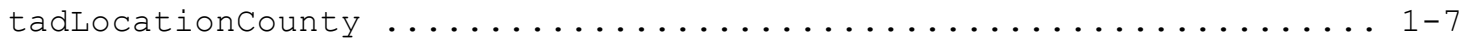

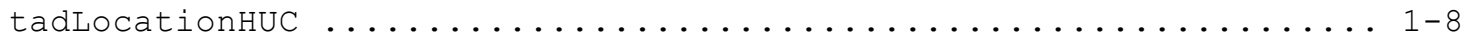

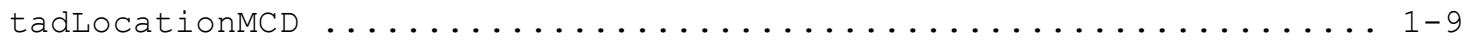

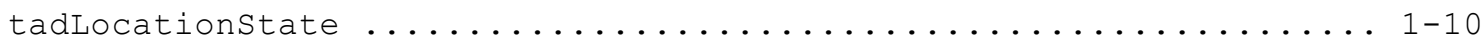

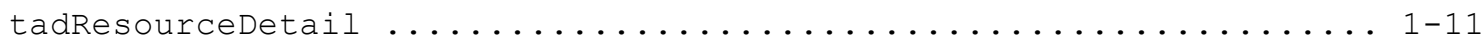

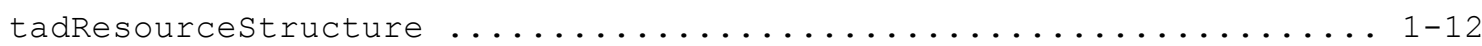

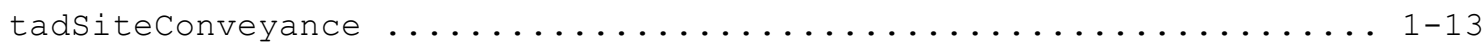

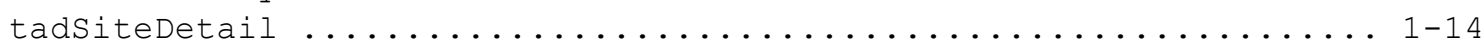

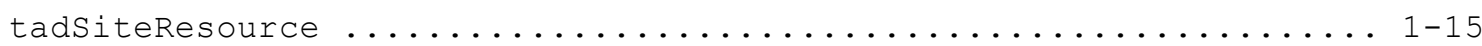

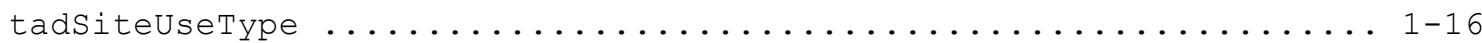

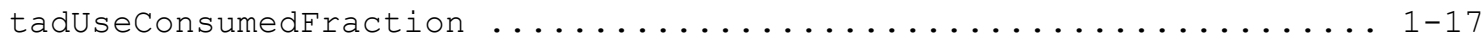

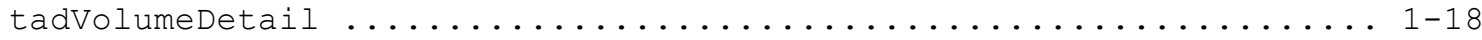

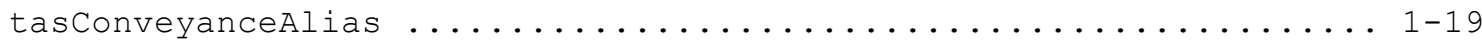

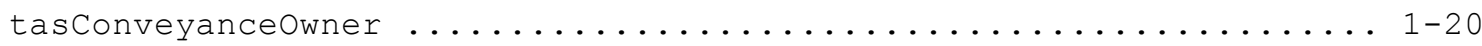

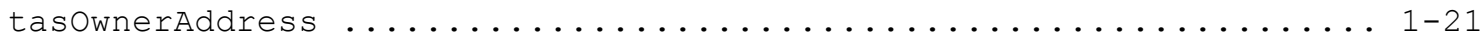

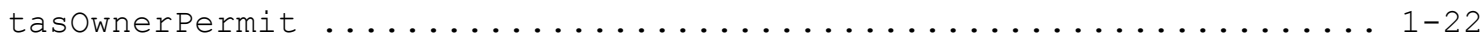

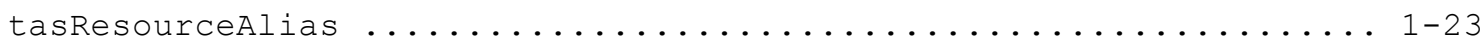

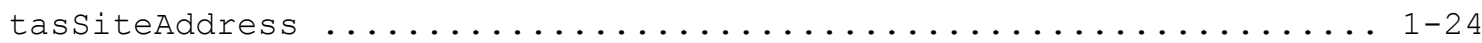

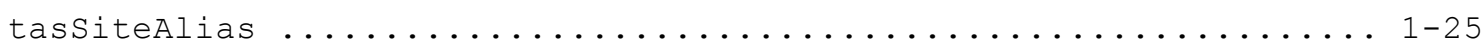

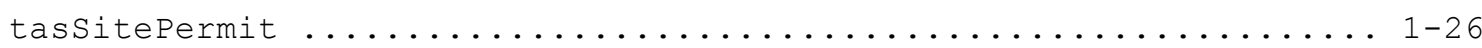

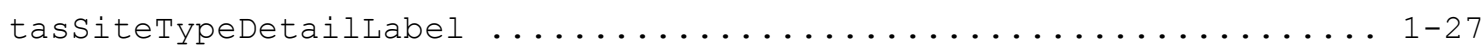

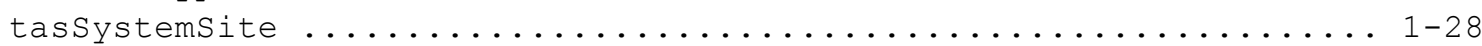

tasWaterBodyDetailLabel .............................. 1-29

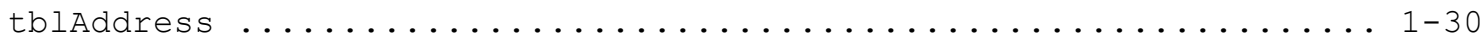

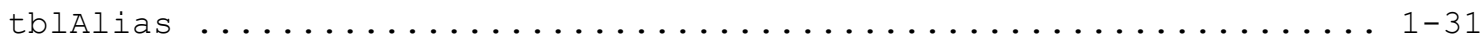

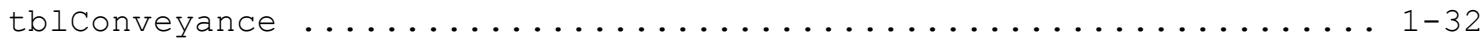

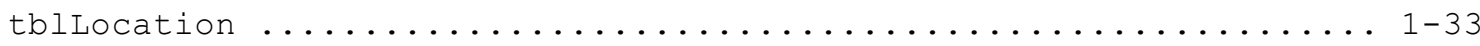

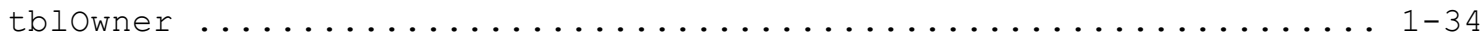

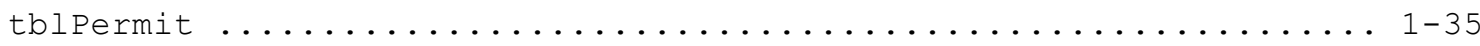

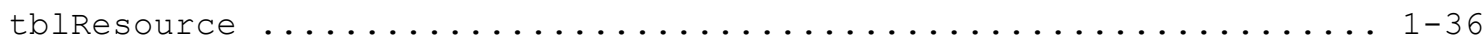

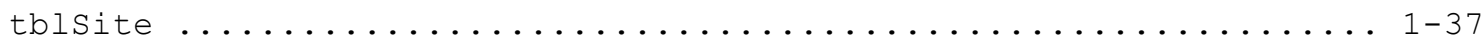

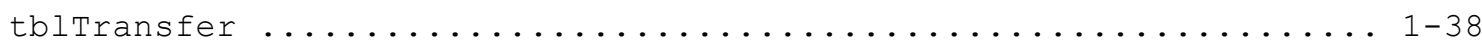

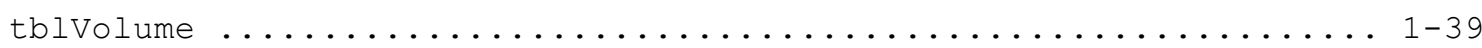

tdsAddressType .................................. 1-40

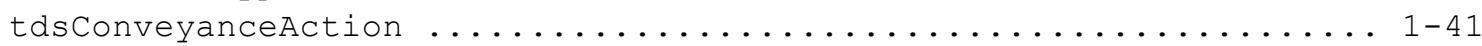

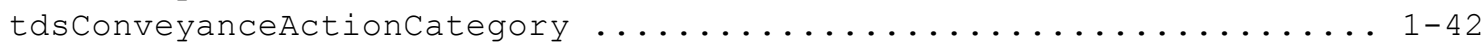

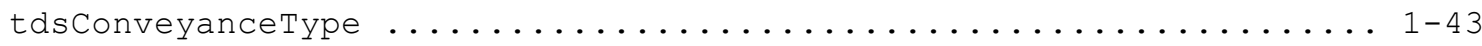

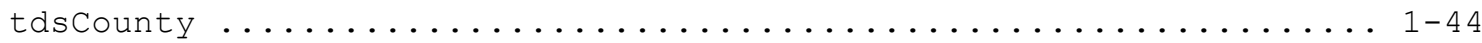

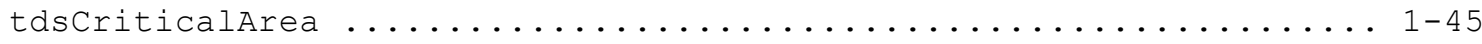

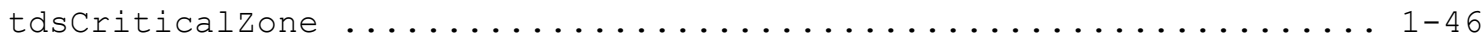

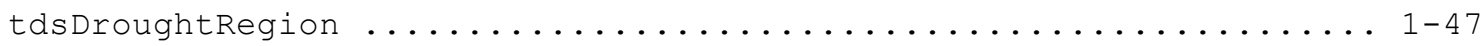

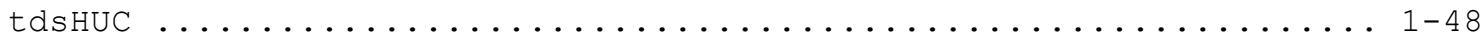

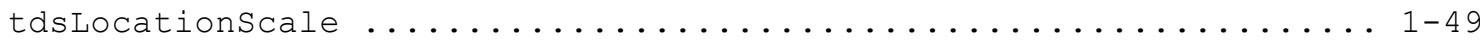

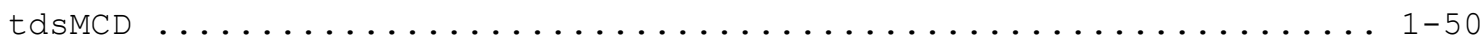

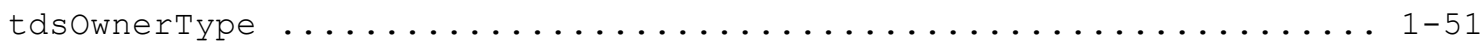

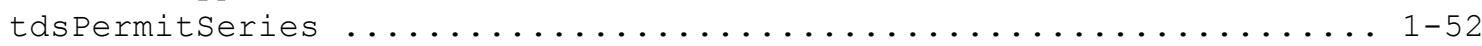

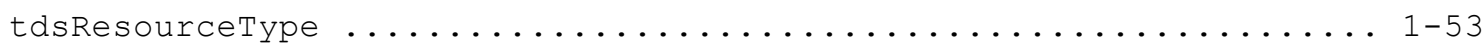

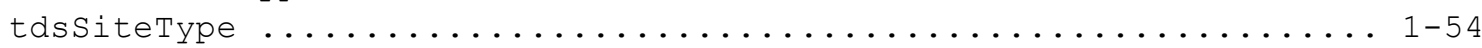

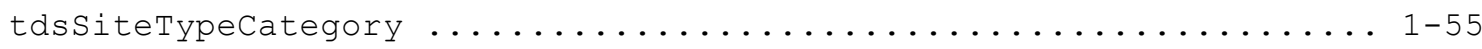

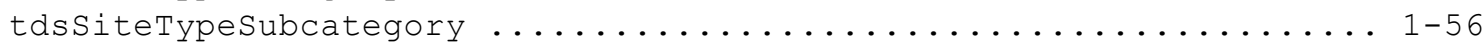

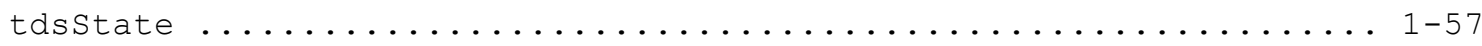


Appendix 1 : Data Dictionary for the New Jersey Water-Transfer Data System (NJWaTr) —Continued

Table Name $\quad$ Page

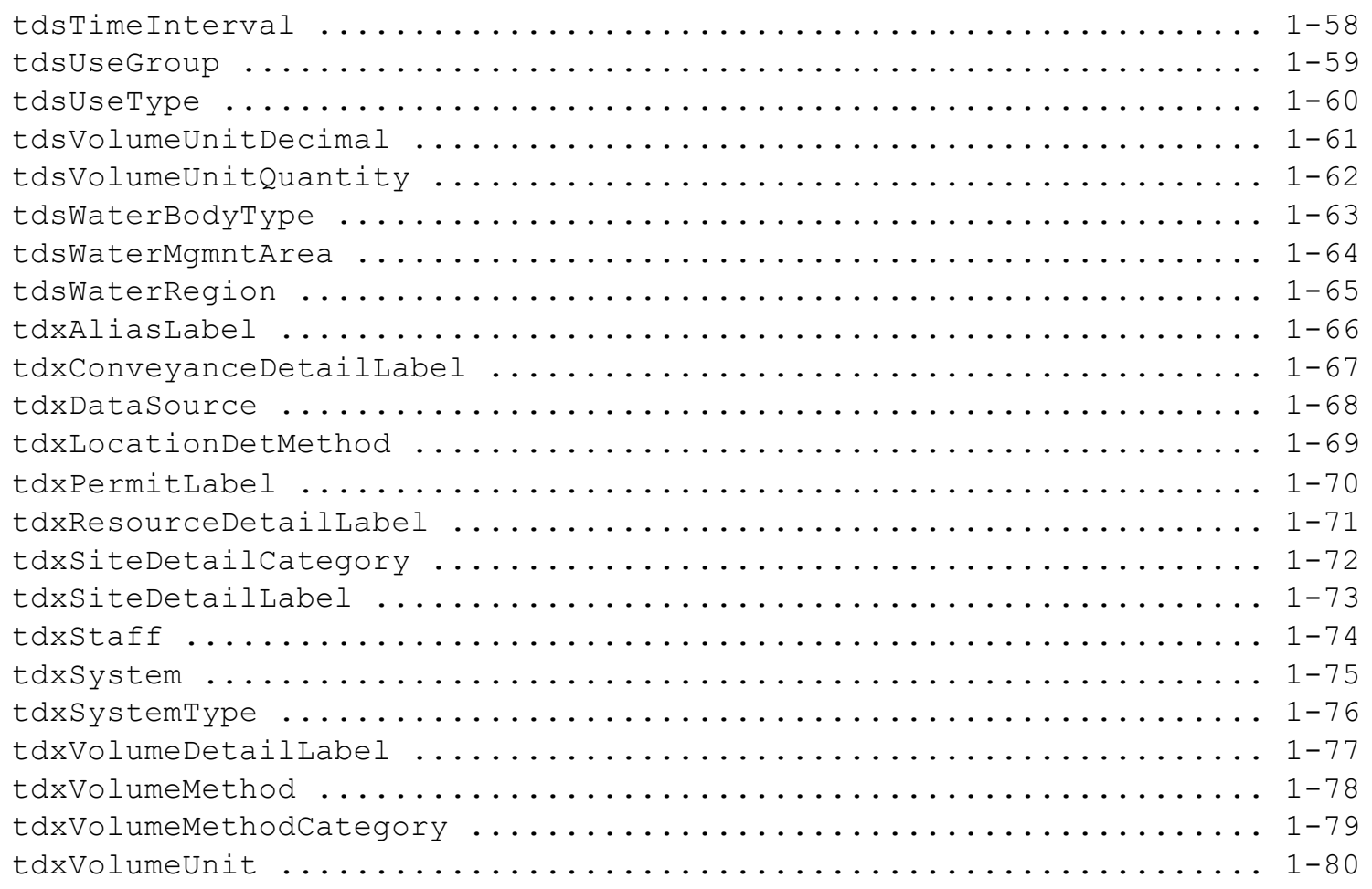


Appendix 1: Data Dictionary for the New Jersey Water-Transfer Data System (NJWaTr) —Continued

\section{Table Name: $\quad$ tadConveyanceDetail}

Table Description: User-defined Detail about a Conveyance

Number of Fields: $\quad 4$

Dependency: Full

Recursive: $\square$

Number of Records $\quad 0$

Loading Order: 4

\begin{tabular}{llllll}
\hline \hline Field Name & PK FK Rqd & Type & Size & Default & Description \\
\hline \hline 1 Conveyance_ID & $\square \square \square$ & Long Integer & 4 & $\begin{array}{l}\text { Key that uniquely } \\
\text { identifies a Conveyance }\end{array}$ \\
2 ConveyanceDetailLabel_ID & $\square \square \square$ & Long Integer & 4 & $\begin{array}{l}\text { Key that uniquely } \\
\text { identifies a Conveyance } \\
\text { Detail Label }\end{array}$ \\
3 ConveyanceDetail & $\square \square \square$ & Text & 50 & $\begin{array}{l}\text { Conveyance Detail } \\
\text { information (numeric or } \\
\text { textual value) } \\
\text { corresponding to the } \\
\text { ConveyanceDetailLabel } \\
\text { descriptor }\end{array}$ \\
& & & & & Note about a \\
Conveyance Detail
\end{tabular}




\section{Table Name: tadConveyanceExpectedFraction}

Table Description: Fraction of originating Sites' available water (not consumed) expected by a receiving Site on a Conveyance for a specified time period

$\begin{array}{lllll}\text { Number of Fields: } & 4 & \text { Dependency: } & \text { Full } & \text { Recursive: } \\ \text { Number of Records } & 0 & \text { Loading Order: } & 4\end{array}$

\begin{tabular}{|c|c|c|c|c|c|c|}
\hline & Field Name & 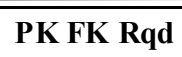 & Type & Size & $\overline{\text { Default }}$ & Description \\
\hline 1 & Conveyance_ID & $\nabla \square \square$ & Long Integer & 4 & & $\begin{array}{l}\text { Key that uniquely } \\
\text { identifies a Conveyance }\end{array}$ \\
\hline 2 & ExpectedFractionEffectiveDate & $\nabla \square \square$ & DateTime & 8 & & $\begin{array}{l}\text { Effective Date for which a } \\
\text { Transfer Expected } \\
\text { Fraction amount applies; } \\
\text { changes to expected } \\
\text { amounts are managed by } \\
\text { new Effective Date } \\
\text { entries with the new } \\
\text { amount }\end{array}$ \\
\hline 3 & ExpectedFractionEndingDate & $\square \square \square$ & DateTime & 8 & & $\begin{array}{l}\text { Ending Date for which a } \\
\text { Percent Transfer } \\
\text { Expected amount applies; } \\
\text { is Null while expected } \\
\text { amount is still in effect }\end{array}$ \\
\hline 4 & ExpectedFraction & $\square \square \square$ & Single & 4 & & $\begin{array}{l}\text { Fraction of originating } \\
\text { Sites' available water } \\
\text { expected by a receiving } \\
\text { Site on a Conveyance; in } \\
\text { decimal units, where } 100 \\
\text { percent is entered as } 1.0\end{array}$ \\
\hline
\end{tabular}


Appendix 1: Data Dictionary for the New Jersey Water-Transfer Data System (NJWaTr) —Continued

\section{Table Name: $\quad$ tadLocationCounty}

Table Description: Association of a proportion of a Location with a County

Number of Fields: $\quad 4$

Dependency: Full

Recursive:

Number of Records 1

Loading Order: 3

\begin{tabular}{|c|c|c|c|c|c|c|}
\hline & Field Name & PK FK Rqd & Type & Size & Default & Description \\
\hline 1 & Location_ID & $\nabla \square \square$ & Long Integer & 4 & & $\begin{array}{l}\text { Key that uniquely } \\
\text { identifies a Location }\end{array}$ \\
\hline 2 & County_ID & $\nabla \square \square$ & Integer & 2 & & $\begin{array}{l}\text { Key that uniquely } \\
\text { identifies a State/County } \\
\text { combination }\end{array}$ \\
\hline 3 & CountyFraction & $\square \square \square$ & Single & 4 & & $\begin{array}{l}\text { Fraction of the Location } \\
\text { that is in the County; in } \\
\text { decimal units, where } 100 \\
\text { percent is entered as } 1.0\end{array}$ \\
\hline 4 & IsPrimaryCounty & $\square \square \square$ & Yes/No & 1 & & $\begin{array}{l}\text { Identifies a primary } \\
\text { County to be associated } \\
\text { with a Location when } \\
\text { more than one may be } \\
\text { associated; when more } \\
\text { than one, one is } \\
\text { designated 'Yes' (the } \\
\text { primary) and the others } \\
\text { 'No'; for singular } \\
\text { Location-County } \\
\text { associations, the value is } \\
\text { always 'Yes' }\end{array}$ \\
\hline
\end{tabular}


Appendix 1: Data Dictionary for the New Jersey Water-Transfer Data System (NJWaTr) —Continued

\section{Table Name: $\quad$ tadLocationHUC}

Table Description: Association of a proportion of a Location with a Hydrologic Unit

Number of Fields: $\quad 4$

Dependency: Full

Recursive: $\square$

Number of Records 1

Loading Order: 4

\begin{tabular}{|c|c|c|c|c|c|c|}
\hline & Field Name & PK FK Rqd & Type & Size & Default & Description \\
\hline 1 & Location_ID & $\nabla \square \square$ & Long Integer & 4 & & $\begin{array}{l}\text { Key that uniquely } \\
\text { identifies a Location }\end{array}$ \\
\hline 2 & HUC_ID & $\nabla \square \square$ & Integer & 2 & & $\begin{array}{l}\text { Key that uniquely } \\
\text { identifies a Hydrologic } \\
\text { Unit }\end{array}$ \\
\hline 3 & HUCFraction & $\square \square \square$ & Single & 4 & & $\begin{array}{l}\text { Fraction of the Location } \\
\text { that is in the HUC; in } \\
\text { decimal units, where } 100 \\
\text { percent is entered as } 1.0\end{array}$ \\
\hline 4 & IsPrimaryHUC & $\square \square \square$ & Yes/No & 1 & & $\begin{array}{l}\text { Identifies a primary HUC } \\
\text { to be associated with a } \\
\text { Location when more than } \\
\text { one may be associated; } \\
\text { when more than one, one } \\
\text { is designated 'Yes' (the } \\
\text { primary) and the others } \\
\text { 'No'; for singular } \\
\text { Location-HUC } \\
\text { associations, the value is } \\
\text { always 'Yes' }\end{array}$ \\
\hline
\end{tabular}


Appendix 1: Data Dictionary for the New Jersey Water-Transfer Data System (NJWaTr) —Continued

\section{Table Name: tadLocationMCD}

Table Description: Association of a proportion of a Location with a Minor Civil Division

Number of Fields: $\quad 4$

Dependency: Full

Recursive:

Number of Records 1

Loading Order: 4

\begin{tabular}{|c|c|c|c|c|c|c|}
\hline & Field Name & PK FK Rqd & Type & Size & Default & Description \\
\hline 1 & Location_ID & $\nabla \square \square$ & Long Integer & 4 & & $\begin{array}{l}\text { Key that uniquely } \\
\text { identifies a Location }\end{array}$ \\
\hline 2 & MCD_ID & $\nabla \square \square$ & Integer & 2 & & $\begin{array}{l}\text { Key that uniquely } \\
\text { identifies a MCD (Minor } \\
\text { Civil Division of US } \\
\text { Census Bureau) }\end{array}$ \\
\hline 3 & MCDFraction & $\square \square \square$ & Single & 4 & & $\begin{array}{l}\text { Fraction of the Location } \\
\text { that is in the MCD; in } \\
\text { decimal units, where } 100 \\
\text { percent is entered as } 1.0\end{array}$ \\
\hline 4 & IsPrimaryMCD & $\square \square \square$ & Yes/No & 1 & & $\begin{array}{l}\text { Identifies a primary MCD } \\
\text { to be associated with a } \\
\text { Location when more than } \\
\text { one may be associated; } \\
\text { when more than one, one } \\
\text { is designated 'Yes' (the } \\
\text { primary) and the others } \\
\text { 'No'; for singular } \\
\text { Location-MCD } \\
\text { associations, the value is } \\
\text { always 'Yes' }\end{array}$ \\
\hline
\end{tabular}


Appendix 1: Data Dictionary for the New Jersey Water-Transfer Data System (NJWaTr) —Continued

\section{Table Name: $\quad$ tadLocationState}

Table Description: Association of a proportion of a Location with a State

Number of Fields: $\quad 4$

Dependency: Full

Recursive: $\square$

Number of Records 1

Loading Order: 3

\begin{tabular}{|c|c|c|c|c|c|c|}
\hline & Field Name & PK FK Rqd & Type & Size & Default & Description \\
\hline 1 & Location_ID & $\nabla \square \square$ & Long Integer & 4 & & $\begin{array}{l}\text { Key that uniquely } \\
\text { identifies a Location }\end{array}$ \\
\hline 2 & State_ID & $\nabla \square \square$ & Integer & 2 & & $\begin{array}{l}\text { Key that uniquely } \\
\text { identifies a State or } \\
\text { Canadian Province }\end{array}$ \\
\hline 3 & StateFraction & $\square \square \square$ & Single & 4 & & $\begin{array}{l}\text { Fraction of the Location } \\
\text { that is in the State; in } \\
\text { decimal units, where } 100 \\
\text { percent is entered as } 1.0\end{array}$ \\
\hline 4 & IsPrimaryState & $\square \square \square$ & Yes/No & 1 & & $\begin{array}{l}\text { Identifies a primary State } \\
\text { to be associated with a } \\
\text { Location when more than } \\
\text { one may be associated; } \\
\text { when more than one, one } \\
\text { is designated 'Yes' (the } \\
\text { primary) and the others } \\
\text { 'No'; for singular } \\
\text { Location-State } \\
\text { associations, the value is } \\
\text { always 'Yes' }\end{array}$ \\
\hline
\end{tabular}




\section{Table Name: $\quad$ tadResourceDetail}

\section{Table Description: User-defined Detail about a Resource (time-dependent)}

Number of Fields: 7

Dependency: Full

Recursive: $\square$

Number of Records $\quad 0$

Loading Order: 4

\begin{tabular}{|c|c|c|c|c|c|c|}
\hline & Field Name & PK FK Rqd & Type & Size & Default & Description \\
\hline 1 & Resource_ID & $\nabla \square \square$ & Long Integer & 4 & & $\begin{array}{l}\text { Key that uniquely } \\
\text { identifies a water } \\
\text { Resource }\end{array}$ \\
\hline 2 & ResourceDetailLabel_ID & $\nabla \square \square$ & Long Integer & 4 & & $\begin{array}{l}\text { Key that uniquely } \\
\text { identifies a Resource } \\
\text { Detail Label }\end{array}$ \\
\hline 3 & ResourceDetailEffectiveDate & $\nabla \square \square$ & DateTime & 8 & & $\begin{array}{l}\text { Effective (starting) date } \\
\text { for a recorded Resource } \\
\text { Detail (lower bound of } \\
\text { time interval) }\end{array}$ \\
\hline 4 & ResourceDetailEndingDate & $\square \square \square$ & DateTime & 8 & & $\begin{array}{l}\text { Ending date for a } \\
\text { recorded Resource Detail } \\
\text { (upper bound of time } \\
\text { interval); is Null while } \\
\text { Detail is still in effect }\end{array}$ \\
\hline 5 & DataSource_ID & $\square \square \square$ & Long Integer & 4 & & $\begin{array}{l}\text { Key that uniquely } \\
\text { identifies a Data Source }\end{array}$ \\
\hline 6 & ResourceDetail & $\square \square \square$ & Text & 50 & & $\begin{array}{l}\text { Resource Detail } \\
\text { information (numeric or } \\
\text { textual value) } \\
\text { corresponding to the } \\
\text { ResourceDetailLabel } \\
\text { descriptor }\end{array}$ \\
\hline 7 & ResourceDetailMemo & $\square \square \square$ & Memo & -- & & $\begin{array}{l}\text { Note about a Resource } \\
\text { Detail }\end{array}$ \\
\hline
\end{tabular}


Appendix 1: Data Dictionary for the New Jersey Water-Transfer Data System (NJWaTr) —Continued

\section{Table Name: $\quad$ tadResourceStructure}

Table Description: Association of a single composite Resource with subparts defined by other Resources

Number of Fields: $\quad 4$

Number of Records $\quad 0$

$$
\begin{array}{ll}
\text { Dependency: } & \text { Full } \\
\text { Loading Order: } & 4
\end{array}
$$

\begin{tabular}{|c|c|c|c|c|c|c|}
\hline & Field Name & PK FK Rqd & Type & Size & Default & Description \\
\hline 1 & ParentResource_ID & $\nabla \square \square$ & Long Integer & 4 & & $\begin{array}{l}\text { Key that uniquely } \\
\text { identifies a water } \\
\text { Resource }\end{array}$ \\
\hline 2 & ChildResource_ID & $\nabla \square \square$ & Long Integer & 4 & & $\begin{array}{l}\text { Key that uniquely } \\
\text { identifies a water } \\
\text { Resource }\end{array}$ \\
\hline 3 & ParentResourceFraction & $\square \square \square$ & Single & 4 & & $\begin{array}{l}\text { Fraction of Parent } \\
\text { Resource represented by } \\
\text { the Child Resource; in } \\
\text { decimal units, where } 100 \\
\text { percent is entered as } 1.0\end{array}$ \\
\hline 4 & IsPrimaryResource & $\square \square \square$ & Yes/No & 1 & No & $\begin{array}{l}\text { Identifies a primary } \\
\text { Resource to be } \\
\text { associated with a } \\
\text { combined Resource; one } \\
\text { may be designated 'Yes' } \\
\text { (the primary) and the } \\
\text { other(s) 'No' }\end{array}$ \\
\hline
\end{tabular}

Recursive: $\square$ 
Appendix 1: Data Dictionary for the New Jersey Water-Transfer Data System (NJWaTr) —Continued

\section{Table Name: $\quad$ tadSiteConveyance}

Table Description: Association of a Site with one end of a Conveyance (From or To)

Number of Fields: 3 Dependency: Full Recursive: $\square$

Number of Records $0 \quad$ Loading Order: 5

\begin{tabular}{llllll}
\hline \hline Field Name & PK FK Rqd & Type & Size & Default & Description \\
\hline \hline 1 & Conveyance_ID & $\square \square \square$ & Long Integer & 4 & $\begin{array}{l}\text { Key that uniquely } \\
\text { identifies a Conveyance }\end{array}$ \\
2 FromOrTo & $\square \square \square$ & Text & 4 & $\begin{array}{l}\text { Identifies an individual } \\
\text { Site as the Input (From) } \\
\text { or Output (To) end of a } \\
\text { specific Conveyance }\end{array}$ \\
3 Site_ID & $\square \square \square$ & Long Integer & 4 & $\begin{array}{l}\text { Key that uniquely } \\
\text { identifies a Site }\end{array}$
\end{tabular}


Appendix 1: Data Dictionary for the New Jersey Water-Transfer Data System (NJWaTr) —Continued

\section{Table Name: $\quad$ tadSiteDetail}

Table Description: User-defined Detail about a Site (time-dependent)

Number of Fields: $\quad 8$

Dependency: Full

Recursive:

Number of Records $\quad 0$

Loading Order: 5

\begin{tabular}{|c|c|c|c|c|c|c|}
\hline & Field Name & PK FK Rqd & Type & Size & Default & Description \\
\hline 1 & Site_ID & $\nabla \square \square$ & Long Integer & 4 & & $\begin{array}{l}\text { Key that uniquely } \\
\text { identifies a Site }\end{array}$ \\
\hline 2 & SiteDetailLabel_ID & $\nabla \square \square$ & Long Integer & 4 & & $\begin{array}{l}\text { Key that uniquely } \\
\text { identifies a Site Detail } \\
\text { Label }\end{array}$ \\
\hline 3 & SiteDetailEffectiveDate & $\nabla \square \square$ & DateTime & 8 & & $\begin{array}{l}\text { Effective (starting) date } \\
\text { for a recorded Site Detail } \\
\text { (lower bound of time } \\
\text { interval) }\end{array}$ \\
\hline 4 & SiteDetailEndingDate & $\square \square \square$ & DateTime & 8 & & $\begin{array}{l}\text { Ending date for a } \\
\text { recorded Site Detail } \\
\text { (upper bound of time } \\
\text { interval); is Null while } \\
\text { Detail is still in effect }\end{array}$ \\
\hline 5 & DataSource_ID & $\square \square \square$ & Long Integer & 4 & & $\begin{array}{l}\text { Key that uniquely } \\
\text { identifies a Data Source }\end{array}$ \\
\hline 6 & TimeInterval_ID & $\square \square \square$ & Integer & 2 & & $\begin{array}{l}\text { Key that uniquely } \\
\text { identifies a Time Interval }\end{array}$ \\
\hline 7 & SiteDetailValue & $\square \square \square$ & Text & 50 & & $\begin{array}{l}\text { Site Detail information } \\
\text { (numeric or textual value) } \\
\text { corresponding to the } \\
\text { SiteDetailLabel descriptor }\end{array}$ \\
\hline 8 & SiteDetailMemo & $\square \square \square$ & Memo & -- & & Note about a Site Detail \\
\hline
\end{tabular}


Appendix 1: Data Dictionary for the New Jersey Water-Transfer Data System (NJWaTr) —Continued

\section{Table Name: $\quad$ tadSiteResource}

Table Description: Association of a Site with a water Resource

Number of Fields: 5 Dependency: Full Recursive: $\square$

Number of Records $\quad 0 \quad$ Loading Order: 5

\begin{tabular}{|c|c|c|c|c|c|c|}
\hline & Field Name & PK FK Rqd & Type & Size & Default & Description \\
\hline 1 & Site_ID & $\nabla \square \square$ & Long Integer & 4 & & $\begin{array}{l}\text { Key that uniquely } \\
\text { identifies a Site }\end{array}$ \\
\hline 2 & Resource_ID & $\square \square \square$ & Long Integer & 4 & & $\begin{array}{l}\text { Key that uniquely } \\
\text { identifies a water } \\
\text { Resource }\end{array}$ \\
\hline 3 & CriticalArea_ID & $\square \square \square$ & Integer & 2 & 0 & $\begin{array}{l}\text { Key that uniquely } \\
\text { identifies a Critical Area }\end{array}$ \\
\hline 4 & CriticalZone_ID & $\square \square \square$ & Integer & 2 & 0 & $\begin{array}{l}\text { Key that uniquely } \\
\text { identifies a Critical Zone }\end{array}$ \\
\hline 5 & ConnectionCount & $\square \square \square$ & Integer & 2 & & $\begin{array}{l}\text { Number of direct } \\
\text { Connections represented } \\
\text { by the association of a } \\
\text { Site with a water Resource }\end{array}$ \\
\hline
\end{tabular}


Appendix 1: Data Dictionary for the New Jersey Water-Transfer Data System (NJWaTr) —Continued

\section{Table Name: $\quad$ tadSiteUseType}

Table Description: Association of a multi-use Site with a UseType and its fraction of the total Use

Number of Fields: $\quad 3$

Number of Records $\quad 0$

$$
\begin{array}{ll}
\text { Dependency: } & \text { Full } \\
\text { Loading Order: } & 5
\end{array}
$$

Recursive:

\begin{tabular}{llllll}
\hline \hline Field Name & PK FK Rqd & Type & Size & Default & Description \\
\hline \hline $1 \quad$ Site_ID & $\square \square \square$ & Long Integer & 4 & $\begin{array}{l}\text { Key that uniquely } \\
\text { identifies a Site }\end{array}$ \\
2 UseType_ID & $\square \square \square$ Integer & 2 & $\begin{array}{l}\text { Key that uniquely } \\
\text { identifies a Water Use } \\
\text { Type classification for a } \\
\text { Site }\end{array}$ \\
& $\square \square \square$ & Single & 4 & $\begin{array}{l}\text { Fraction of total Use for a } \\
\text { multi-use Site represented } \\
\text { by an individual } \\
\text { UseType; in decimal } \\
\text { units, where 100 percent } \\
\text { is entered as 1.0 }\end{array}$ \\
& & & & \\
& & & &
\end{tabular}


Appendix 1: Data Dictionary for the New Jersey Water-Transfer Data System (NJWaTr) —Continued

\section{Table Name: $\quad$ tadUseConsumedFraction}

Table Description: Association of a UseType with monthly estimates of the fraction of available water that is consumed

Number of Fields: $\quad 3$

Dependency: Full

Recursive: $\square$

Number of Records 12

Loading Order: 3

\begin{tabular}{|c|c|c|c|c|c|c|}
\hline & Field Name & PK FK Rqd & Type & Size & Default & Description \\
\hline 1 & UseType_ID & $\nabla \square \square$ & Integer & 2 & & $\begin{array}{l}\text { Key that uniquely } \\
\text { identifies a Water Use } \\
\text { Type classification for a } \\
\text { Site }\end{array}$ \\
\hline 2 & Month & $\square \square \square$ & Long Integer & 4 & & $\begin{array}{l}\text { Month of the year, in } \\
\text { Integer format (Jan = } 1 \text {, } \\
\text { Feb }=2 \text {, and so forth) }\end{array}$ \\
\hline 3 & ConsumedFraction & $\square \square \square$ & Single & 4 & & $\begin{array}{l}\text { Fraction of the UseType } \\
\text { water that is consumed in } \\
\text { a given Month; in } \\
\text { decimal units, where } 100 \\
\text { percent is entered as } 1.0\end{array}$ \\
\hline
\end{tabular}


Appendix 1: Data Dictionary for the New Jersey Water-Transfer Data System (NJWaTr) —Continued

\section{Table Name: $\quad$ tadVolumeDetail}

Table Description: User-defined Detail about a Transfer Volume

Number of Fields: $\quad 4$

Dependency: Full

Recursive:

Number of Records $\quad 0$

Loading Order: 6

\begin{tabular}{llllll}
\hline \hline Field Name & PK FK Rqd & Type & Size & Default & Description \\
\hline \hline 1 Volume_ID & $\square \square \square$ & Long Integer & 4 & $\begin{array}{l}\text { Key that uniquely } \\
\text { identifies a Volume for a } \\
\text { Transfer }\end{array}$ \\
2 VolumeDetailLabel_ID & $\square \square \square$ Long Integer & 4 & $\begin{array}{l}\text { Key that uniquely } \\
\text { identifies a Volume Detail } \\
\text { Label }\end{array}$ \\
3 VolumeDetail & $\square \square \square$ & Text & & $\begin{array}{l}\text { Volume Detail information } \\
\text { (numeric or textual value) } \\
\text { corresponding to the }\end{array}$ \\
& & & 50 & $\begin{array}{l}\text { VolumeDetailLabel } \\
\text { descriptor }\end{array}$ \\
& & & & $\begin{array}{l}\text { Note about a Volume } \\
\text { Detail }\end{array}$
\end{tabular}


Appendix 1: Data Dictionary for the New Jersey Water-Transfer Data System (NJWaTr) — Continued

\section{Table Name: $\quad$ tasConveyanceAlias}

Table Description: Association of a Conveyance with an Alias

Number of Fields: 2 Dependency: Full Recursive: $\square$

Number of Records $0 \quad$ Loading Order: 6

\begin{tabular}{llclcl}
\hline \hline Field Name & PK FK Rqd & Type & Size & Default & Description \\
\hline \hline 1 & Conveyance_ID & $\square \square \square$ & Long Integer & 4 & $\begin{array}{l}\text { Key that uniquely } \\
\text { identifies a Conveyance }\end{array}$ \\
2 & Alias_ID & $\square \square \square$ & Long Integer & 4 & $\begin{array}{l}\text { Key that uniquely } \\
\text { identifies an Alias }\end{array}$
\end{tabular}


Appendix 1: Data Dictionary for the New Jersey Water-Transfer Data System (NJWaTr) — Continued

\section{Table Name: $\quad$ tasConveyanceOwner}

Table Description: Association of a Conveyance with an Owner

Number of Fields: 2 Dependency: Full Recursive: $\square$

Number of Records $\quad 0 \quad$ Loading Order: 4

\begin{tabular}{llclcl}
\hline \hline Field Name & PK FK Rqd & Type & Size & Default & Description \\
\hline \hline 1 & Conveyance_ID & $\square \square \square$ & Long Integer & 4 & $\begin{array}{l}\text { Key that uniquely } \\
\text { identifies a Conveyance }\end{array}$ \\
2 & Owner_ID & $\square \square \square$ & Long Integer & 4 & $\begin{array}{l}\text { Key that uniquely } \\
\text { identifies an Owner }\end{array}$
\end{tabular}


Appendix 1: Data Dictionary for the New Jersey Water-Transfer Data System (NJWaTr) — Continued

\section{Table Name: $\quad$ tasOwnerAddress}

Table Description: Association of an Owner with an Address

Number of Fields: $\quad 2$

Dependency: Full

Recursive: $\square$

Number of Records 0

Loading Order: 3

\begin{tabular}{llclcl}
\hline \hline Field Name & PK FK Rqd & Type & Size & Default & Description \\
\hline \hline 1 & Owner_ID & $\square \square \square$ & Long Integer & 4 & $\begin{array}{l}\text { Key that uniquely } \\
\text { identifies an Owner }\end{array}$ \\
2 & Address_ID & $\square \square \square$ & Long Integer & 4 & $\begin{array}{l}\text { Key that uniquely } \\
\text { identifies an Address }\end{array}$
\end{tabular}


Appendix 1: Data Dictionary for the New Jersey Water-Transfer Data System (NJWaTr) — Continued

\section{Table Name: $\quad$ tasOwnerPermit}

Table Description: Association of an Owner with a Permit

Number of Fields: 2 Dependency: Full

Number of Records $0 \quad$ Loading Order: 6

\begin{tabular}{llclcl}
\hline \hline Field Name & PK FK Rqd & Type & Size & Default & Description \\
\hline \hline 1 & Owner_ID & $\square \square \square$ & Long Integer & 4 & $\begin{array}{l}\text { Key that uniquely } \\
\text { identifies an Owner }\end{array}$ \\
2 & Permit_ID & $\square \square \square$ & Long Integer & 4 & $\begin{array}{l}\text { Key that uniquely } \\
\text { identifies a Permit }\end{array}$
\end{tabular}


Appendix 1: Data Dictionary for the New Jersey Water-Transfer Data System (NJWaTr) — Continued

\section{Table Name: $\quad$ tasResourceAlias}

Table Description: Association of a water Resource with an Alias

Number of Fields: 2 Dependency: Full Recursive: $\square$

Number of Records $0 \quad$ Loading Order: 6

\begin{tabular}{|c|c|c|c|c|c|c|}
\hline & Field Name & PK FK Rqd & Type & Size & Default & Description \\
\hline 1 & Resource_ID & $\nabla \square \square$ & Long Integer & 4 & & $\begin{array}{l}\text { Key that uniquely } \\
\text { identifies a water } \\
\text { Resource }\end{array}$ \\
\hline 2 & Alias_ID & $\nabla \square \square$ & Long Integer & 4 & & $\begin{array}{l}\text { Key that uniquely } \\
\text { identifies an Alias }\end{array}$ \\
\hline
\end{tabular}


Appendix 1: Data Dictionary for the New Jersey Water-Transfer Data System (NJWaTr) — Continued

\section{Table Name: $\quad$ tasSiteAddress}

Table Description: Association of a Site with an Address

Number of Fields: 2 Dependency: Full

Recursive: $\square$

Number of Records $0 \quad$ Loading Order: 5

\begin{tabular}{|c|c|c|c|c|c|c|}
\hline & Field Name & PK FK Rqd & Type & Size & Default & Description \\
\hline 1 & Site_ID & $\nabla \nabla \square$ & Long Integer & 4 & & $\begin{array}{l}\text { Key that uniquely } \\
\text { identifies a Site }\end{array}$ \\
\hline 2 & Address_ID & $\nabla \square \square$ & Long Integer & 4 & & $\begin{array}{l}\text { Key that uniquely } \\
\text { identifies an Address }\end{array}$ \\
\hline
\end{tabular}


Appendix 1: Data Dictionary for the New Jersey Water-Transfer Data System (NJWaTr) — Continued

\section{Table Name: $\quad$ tasSiteAlias}

Table Description: Association of a Site with an Alias

Number of Fields: 2 Dependency: Full Recursive: $\square$

Number of Records $0 \quad$ Loading Order: 6

\begin{tabular}{lccccc}
\hline \hline Field Name & PK FK Rqd & Type & Size & Default & Description \\
\hline \hline
\end{tabular}

1 Site_ID $\quad \square \square \square\left(\begin{array}{llll}\text { Long Integer } & 4 & \begin{array}{l}\text { Key that uniquely } \\ \text { identifies a Site }\end{array}\end{array}\right.$

2 Alias_ID $\square \square \square$ Long Integer $\quad 4 \quad$ Key that uniquely identifies an Alias 
Appendix 1: Data Dictionary for the New Jersey Water-Transfer Data System (NJWaTr) — Continued

\section{Table Name: $\quad$ tasSitePermit}

Table Description: Association of a Site with a Permit

Number of Fields: 2 Dependency: Full Recursive: $\square$

Number of Records $0 \quad$ Loading Order: 6

\begin{tabular}{|c|c|c|c|c|c|c|}
\hline & Field Name & PK FK Rqd & Type & Size & Default & Description \\
\hline 1 & Site_ID & $\nabla \nabla \square$ & Long Integer & 4 & & $\begin{array}{l}\text { Key that uniquely } \\
\text { identifies a Site }\end{array}$ \\
\hline 2 & Permit_ID & $\nabla \square \square$ & Long Integer & 4 & & $\begin{array}{l}\text { Key that uniquely } \\
\text { identifies a Permit }\end{array}$ \\
\hline
\end{tabular}


Appendix 1: Data Dictionary for the New Jersey Water-Transfer Data System (NJWaTr) — Continued

\section{Table Name: $\quad$ tasSiteTypeDetailLabel}

Table Description: Association of a SiteType with a specific SiteDetailLabel for the purpose of controlling the list of valid labels for different site types

Number of Fields: 2 Dependency: Full Recursive:

Number of Records $\quad 0 \quad$ Loading Order: 4

\begin{tabular}{|c|c|c|c|c|c|}
\hline Field Name & PK FK Rqd & Type & Size & Default & Description \\
\hline
\end{tabular}

1 SiteDetailLabel_ID $\quad \square \square \square$ Long Integer $\quad 4 \quad$ Key that uniquely identifies a Site Detail Label

2 SiteType_ID $\square \square \square$ Integer $\quad \square \quad 2 \quad$ Key that uniquely identifies a Site Type 
Appendix 1: Data Dictionary for the New Jersey Water-Transfer Data System (NJWaTr) — Continued

\section{Table Name: $\quad$ tasSystemSite}

Table Description: Association of a Site with a named System

Number of Fields: 2 Dependency: Full Recursive: $\square$

Number of Records $0 \quad$ Loading Order: 5

\begin{tabular}{|c|c|c|c|c|c|c|}
\hline & Field Name & PK FK Rqd & Type & Size & Default & Description \\
\hline 1 & System_ID & $\nabla \nabla \square$ & Long Integer & 4 & & $\begin{array}{l}\text { Key that uniquely } \\
\text { identifies a System }\end{array}$ \\
\hline 2 & Site_ID & $\nabla \square \square$ & Long Integer & 4 & & $\begin{array}{l}\text { Key that uniquely } \\
\text { identifies a Site }\end{array}$ \\
\hline
\end{tabular}


Appendix 1: Data Dictionary for the New Jersey Water-Transfer Data System (NJWaTr) — Continued

\section{Table Name: $\quad$ tasWaterBodyDetailLabel}

Table Description: Association of a WaterBodyType with a specific ResourceDetailLabel for the purpose of controlling the list of valid labels for different water body types

Number of Fields: $\quad 2$

Dependency: Full

Recursive: $\square$

Number of Records $\quad 0$

Loading Order: 3

\begin{tabular}{llllll}
\hline \hline Field Name & PK FK Rqd & Type & Size & Default & Description \\
\hline \hline 1 & WaterBodyType_ID & $\square \square \square$ & Integer & 2 & $\begin{array}{l}\text { Key that uniquely } \\
\text { identifies a Type of }\end{array}$ \\
& & & $\begin{array}{l}\text { Water Body represented } \\
\text { by a water Resource (for } \\
\text { example, lake, river, } \\
\text { estuary) }\end{array}$ \\
& & & & $\begin{array}{l}\text { Key that uniquely } \\
\text { identifies a Resource } \\
\text { Detail Label }\end{array}$ \\
& ResourceDetailLabel_ID & $\square \square \square$ & Long Integer & 4 &
\end{tabular}


Appendix 1: Data Dictionary for the New Jersey Water-Transfer Data System (NJWaTr) —Continued

\section{Table Name: $\quad$ tblAddress}

Table Description: Address information for an Owner or Site

Number of Fields: 9 Dependency: Partial Recursive: $\square$

Number of Records $0 \quad$ Loading Order: 2

\begin{tabular}{|c|c|c|c|c|c|c|}
\hline & Field Name & PK FK Rqd & Type & Size & Default & Description \\
\hline 1 & Address_ID & $\nabla \square \square$ & $\begin{array}{l}\text { AutoNumber } \\
\text { (Long) }\end{array}$ & 4 & & $\begin{array}{l}\text { Key that uniquely } \\
\text { identifies an Address }\end{array}$ \\
\hline 2 & AddressType_ID & $\square \square \square$ & Integer & 2 & & $\begin{array}{l}\text { Key that uniquely } \\
\text { identifies an Address } \\
\text { Type }\end{array}$ \\
\hline 3 & AddressLine1 & $\square \square \square$ & Text & 50 & & Address Line 1 \\
\hline 4 & AddressLine2 & $\square \square \square$ & Text & 50 & & Address Line 2 \\
\hline 5 & City & $\square \square \square$ & Text & 50 & & City Name \\
\hline 6 & StateAbbrv & $\square \square \square$ & Text & 2 & & $\begin{array}{l}\text { State (or Canadian } \\
\text { Province) Abbreviation }\end{array}$ \\
\hline 7 & ZipCode & $\square \square \square$ & Text & 10 & & Postal Zip Code \\
\hline 8 & CountryAbbrv & $\square \square \square$ & Text & 3 & "USA" & $\begin{array}{l}\text { Country Abbreviation } \\
\text { (USA or CAN) }\end{array}$ \\
\hline 9 & AddressMemo & $\square \square \square$ & Memo & -- & & Note about an Address \\
\hline
\end{tabular}


Appendix 1: Data Dictionary for the New Jersey Water-Transfer Data System (NJWaTr) — Continued

\section{Table Name: $\quad$ tblAlias}

Table Description: $\quad$ Alias, such as a local or Federal ID code, for a Site, Conveyance or water Resource

Number of Fields: $\quad 4$

Number of Records $\quad 0$

$$
\begin{array}{ll}
\text { Dependency: } & \text { Partial } \\
\text { Loading Order: } & 5
\end{array}
$$

\begin{tabular}{|c|c|c|c|c|c|c|}
\hline & Field Name & PK FK Rqd & Type & Size & Default & Description \\
\hline 1 & Alias_ID & $\nabla \square \square$ & $\begin{array}{l}\text { AutoNumber } \\
\text { (Long) }\end{array}$ & 4 & & $\begin{array}{l}\text { Key that uniquely } \\
\text { identifies an Alias }\end{array}$ \\
\hline 2 & AliasLabel_ID & $\square \square \square$ & Long Integer & 4 & & $\begin{array}{l}\text { Key that uniquely } \\
\text { identifies an AliasLabel }\end{array}$ \\
\hline 3 & AliasValue & $\square \square \square$ & Text & 50 & & $\begin{array}{l}\text { Alias Value, such as a } \\
\text { Station ID }\end{array}$ \\
\hline 4 & AliasMemo & $\square \square \square$ & Memo & -- & & Note about an Alias \\
\hline
\end{tabular}

Recursive: $\square$ 
Appendix 1: Data Dictionary for the New Jersey Water-Transfer Data System (NJWaTr) — Continued

\section{Table Name: tblConveyance}

Table Description: Conveyance that serves as the water-carrying connection between two Sites (unidirectional); includes pipes, canals and conduits, trucking, and virtual linkages

Number of Fields: $\quad 5$

Dependency: Partial

Loading Order: 3

Recursive:

Number of Records 0

\begin{tabular}{|c|c|c|c|c|c|c|}
\hline & Field Name & $\overline{~ P K ~ F K ~ R q d ~}$ & Type & Size & Default & Description \\
\hline 1 & Conveyance_ID & $\nabla \square \square$ & $\begin{array}{l}\text { AutoNumber } \\
\text { (Long) }\end{array}$ & 4 & & $\begin{array}{l}\text { Key that uniquely } \\
\text { identifies a Conveyance }\end{array}$ \\
\hline 2 & ConveyanceType_ID & $\square \square \square$ & Integer & 2 & & $\begin{array}{l}\text { Key that uniquely } \\
\text { identifies a Conveyance } \\
\text { Type }\end{array}$ \\
\hline 3 & ConveyanceAction_ID & $\square \square \square$ & Integer & 2 & & $\begin{array}{l}\text { Key that uniquely } \\
\text { identifies an Action } \\
\text { performed by a } \\
\text { Conveyance between two } \\
\text { Sites }\end{array}$ \\
\hline 4 & ConveyanceName & $\square \square \square$ & Text & 200 & & Name of Conveyance \\
\hline 5 & ConveyanceMemo & $\square \square \square$ & Memo & -- & & Note about a Conveyance \\
\hline
\end{tabular}


Appendix 1: Data Dictionary for the New Jersey Water-Transfer Data System (NJWaTr) — Continued

\section{Table Name: $\quad$ tblLocation}

Table Description: Location information for a Site, including scale, coordinates, and association with geopolitical and hydrologic areas

Number of Fields: $\quad 9$

Dependency: Partial

Recursive: $\square$

Number of Records 1

Loading Order: 2

\begin{tabular}{|c|c|c|c|c|c|c|}
\hline & Field Name & PK FK Rqd & Type & Size & Default & Description \\
\hline 1 & Location_ID & $\nabla \square \square$ & $\begin{array}{l}\text { AutoNumber } \\
\text { (Long) }\end{array}$ & 4 & & $\begin{array}{l}\text { Key that uniquely } \\
\text { identifies a Location }\end{array}$ \\
\hline 2 & LocationDetMethod_ID & $\square \square \square$ & Long Integer & 4 & & $\begin{array}{l}\text { Key that uniquely } \\
\text { identifies a Location } \\
\text { Determination Method }\end{array}$ \\
\hline 3 & LocationScale_ID & $\square \square \square$ & Integer & 2 & & $\begin{array}{l}\text { Key that uniquely } \\
\text { identifies a spatial Scale } \\
\text { term for a Location }\end{array}$ \\
\hline 4 & LocationName & $\square \square \square$ & Text & 200 & & Location Name \\
\hline 5 & LocationLatitude & $\square \square \square$ & Text & 30 & & $\begin{array}{l}\text { Latitude coordinate } \\
\text { (DMS format) for a } \\
\text { Location }\end{array}$ \\
\hline 6 & LocationLongitude & $\square \square \square$ & Text & 30 & & $\begin{array}{l}\text { Longitude coordinate } \\
\text { (DMS format) for a } \\
\text { Location }\end{array}$ \\
\hline 7 & NJEasting & $\square \square \square$ & Text & 50 & & $\begin{array}{l}\text { New Jersey system East } \\
\text { coordinate }\end{array}$ \\
\hline 8 & NJNorthing & $\square \square \square$ & Text & 50 & & $\begin{array}{l}\text { New Jersey system North } \\
\text { coordinate }\end{array}$ \\
\hline 9 & LocationMemo & $\square \square \square$ & Memo & -- & & Note about a Location \\
\hline
\end{tabular}


Appendix 1: Data Dictionary for the New Jersey Water-Transfer Data System (NJWaTr) — Continued

\section{Table Name: $\quad$ tblOwner}

Table Description: Owner organization or entity that owns, controls or monitors a Site, Conveyance or water Resource, or is the parent organization for a Data Source

Number of Fields: 7

Number of Records $\quad 4$

$$
\begin{array}{lll}
\text { Dependency: } & \text { Partial } & \text { Recursive: } \square \\
\text { Loading Order: } & 2 &
\end{array}
$$

\begin{tabular}{|c|c|c|c|c|c|c|}
\hline & Field Name & PK FK Rqd & Type & Size & Default & Description \\
\hline 1 & Owner_ID & $\square \square \square$ & $\begin{array}{l}\text { AutoNumber } \\
\text { (Long) }\end{array}$ & 4 & & $\begin{array}{l}\text { Key that uniquely } \\
\text { identifies an Owner }\end{array}$ \\
\hline 2 & OwnerType_ID & $\square \square \square$ & Integer & 2 & & $\begin{array}{l}\text { Key that uniquely } \\
\text { identifies an Owner Type }\end{array}$ \\
\hline 3 & ParentOwner_ID & $\square \square \square$ & Long Integer & 4 & & $\begin{array}{l}\text { Key that uniquely } \\
\text { identifies the Parent- } \\
\text { Owner of the current } \\
\text { Owner, for example a } \\
\text { regional organization }\end{array}$ \\
\hline 4 & OwnerName & $\square \square \square$ & Text & 200 & & Owner Name \\
\hline 5 & OwnerContact & $\square \square \square$ & Text & 200 & & $\begin{array}{l}\text { Contact Name for an } \\
\text { Owner }\end{array}$ \\
\hline 6 & OwnerPhone & $\square \square \square$ & Text & 25 & & $\begin{array}{l}\text { Contact Phone number } \\
\text { for an Owner }\end{array}$ \\
\hline 7 & OwnerMemo & $\square \square \square$ & Memo & -- & & Note about an Owner \\
\hline
\end{tabular}


Appendix 1: Data Dictionary for the New Jersey Water-Transfer Data System (NJWaTr) —Continued

\section{Table Name: $\quad$ tblPermit}

Table Description: Permit, such as PWSID or BWA, assigned to a Site or Owner by a permitting agency

Number of Fields: $\quad 5$

Number of Records 0

$$
\begin{array}{ll}
\text { Dependency: } & \text { Partial } \\
\text { Loading Order: } & 5
\end{array}
$$

Recursive: $\square$

\begin{tabular}{llllll}
\hline \hline Field Name & PK FK Rqd & Type & Size & Default & Description \\
\hline \hline 1 & Permit_ID & $\square \square \square$ & $\begin{array}{l}\text { AutoNumber } \\
\text { (Long) }\end{array}$ & 4 & $\begin{array}{l}\text { Key that uniquely } \\
\text { identifies a Permit }\end{array}$ \\
2 & PermitLabel_ID & $\square \square \square$ & Long Integer & 4 & $\begin{array}{l}\text { Key that uniquely } \\
\text { identifies a Permit Label }\end{array}$ \\
3 & PermitSeries_ID & $\square \square \square$ & Integer & 2 & $\begin{array}{l}\text { Key that uniquely } \\
\text { identifies a Permit Series }\end{array}$ \\
4 & PermitNumber & $\square \square \square$ & Text & 50 & $\begin{array}{l}\text { Code or Number of a } \\
\text { specific Permit }\end{array}$ \\
5 & PermitMemo & $\square \square \square$ & Memo & -- & Memo about a Permit
\end{tabular}




\section{Table Name: $\quad$ tblResource}

Table Description: Water Resource involved in water-use activities (withdrawals, transfers, and returns); includes aquifers, springs, streams and rivers, lakes and reservoirs, estuaries and embayments, and the ocean

Number of Fields: 6

Dependency: Partial

Recursive: $\square$

Number of Records 1

Loading Order: 3

\begin{tabular}{|c|c|c|c|c|c|c|}
\hline & Field Name & PK FK Rqd & Type & Size & Default & Description \\
\hline 1 & Resource_ID & $\square \square \square$ & $\begin{array}{l}\text { AutoNumber } \\
\text { (Long) }\end{array}$ & 4 & & $\begin{array}{l}\text { Key that uniquely } \\
\text { identifies a water } \\
\text { Resource }\end{array}$ \\
\hline 2 & WaterBodyType_ID & $\square \square \square$ & Integer & 2 & & $\begin{array}{l}\text { Key that uniquely } \\
\text { identifies a Type of } \\
\text { Water Body represented } \\
\text { by a water Resource (for } \\
\text { example, lake, river, } \\
\text { estuary) }\end{array}$ \\
\hline 3 & Owner_ID & $\square \square \square$ & Long Integer & 4 & & $\begin{array}{l}\text { Key that uniquely } \\
\text { identifies an Owner }\end{array}$ \\
\hline 4 & ResourceName & $\square \square \square$ & Text & 200 & & $\begin{array}{l}\text { Name typically used to } \\
\text { identify a specific water } \\
\text { Resource (for example, } \\
\text { 'Mill Creek' or 'Spruce } \\
\text { Run Reservoir') }\end{array}$ \\
\hline 5 & ResourceCodeName & $\square \square \square$ & Text & 200 & & $\begin{array}{l}\text { Code-Name phrase used } \\
\text { to uniquely identify a } \\
\text { Resource to distinguish it } \\
\text { from others having the } \\
\text { same ResourceName (for } \\
\text { example, 'Mill Creek, } \\
\text { Wildwood Crest, NJ' } \\
\text { versus 'Mill Creek, Ocean } \\
\text { City, NJ') }\end{array}$ \\
\hline 6 & ResourceMemo & $\square \square \square$ & Memo & -- & & $\begin{array}{l}\text { Note about a water } \\
\text { Resource }\end{array}$ \\
\hline
\end{tabular}




\section{Table Name: tblSite}

Table Description: Site involved in water-use activities; includes wells, intakes and outfalls, distribution and treatment facilities, collection systems, industrial, commercial and domestic users, and aggregate-use entities

Number of Fields: $\quad 8$

Number of Records 3

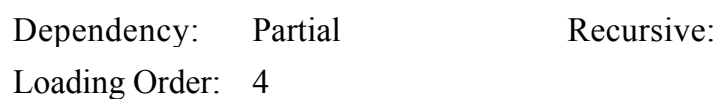

\begin{tabular}{llllll}
\hline \hline Field Name & PK FK Rqd & Type & Size & Default & Description \\
\hline \hline 1 & Site_ID & $\square \square \square$ & $\begin{array}{l}\text { AutoNumber } \\
\text { (Long) }\end{array}$ & 4 & $\begin{array}{l}\text { Key that uniquely } \\
\text { identifies a Site }\end{array}$ \\
2 & Location_ID & $\square \square \square$ & $\begin{array}{l}\text { Long Integer } \\
\text { Key that uniquely } \\
\text { identifies a Location }\end{array}$ \\
3 & Owner_ID & $\square \square \square$ & Long Integer & 4 & $\begin{array}{l}\text { Key that uniquely } \\
\text { identifies an Owner }\end{array}$ \\
4 & SiteType_ID & $\square \square \square$ & Integer & 2 & $\begin{array}{l}\text { Key that uniquely } \\
\text { identifies a Site Type }\end{array}$ \\
5 & UseType_ID & $\square \square \square$ & Integer & 2 & $\begin{array}{l}\text { Key that uniquely } \\
\text { identifies a Water Use } \\
\text { Type classification for a } \\
\text { Site }\end{array}$ \\
7 & SiteName & $\square$ & & & Site Name \\
7 & SiteContact & $\square \square$ & Text & 100 & Site Contact Information \\
8 & SiteMemo & $\square \square \square$ & Memo & -- & Note about a Site
\end{tabular}




\title{
Table Name: $\quad$ tblTransfer
}

Table Description: Record of a water Transfer through a Conveyance between two Sites for a specified Time Interval

Number of Fields: $\quad 7$

Number of Records 0

\author{
Dependency: Partial \\ Loading Order: 4
}

Recursive: $\square$

\begin{tabular}{|c|c|c|c|c|c|c|}
\hline & Field Name & PK FK Rqd & Type & Size & Default & Description \\
\hline 1 & Transfer_ID & $\nabla \square \square$ & $\begin{array}{l}\text { AutoNumber } \\
\text { (Long) }\end{array}$ & 4 & & $\begin{array}{l}\text { Key that uniquely } \\
\text { identifies a Transfer }\end{array}$ \\
\hline 2 & Conveyance_ID & $\square \square \square$ & Long Integer & 4 & & $\begin{array}{l}\text { Key that uniquely } \\
\text { identifies a Conveyance }\end{array}$ \\
\hline 3 & TimeInterval_ID & $\square \square \square$ & Integer & 2 & & $\begin{array}{l}\text { Key that uniquely } \\
\text { identifies a Time Interval }\end{array}$ \\
\hline 4 & TransferEffectiveDate & $\square \square \square$ & DateTime & 8 & & $\begin{array}{l}\text { Effective (starting) date } \\
\text { for a Transfer record } \\
\text { (lower bound of time } \\
\text { interval) }\end{array}$ \\
\hline 5 & TransferEndingDate & $\square \square \square$ & DateTime & 8 & & $\begin{array}{l}\text { Ending date for a } \\
\text { Transfer record (upper } \\
\text { bound of time interval) }\end{array}$ \\
\hline 6 & VolumeMG & $\square \square \square$ & Double & 8 & -1 & $\begin{array}{l}\text { Volume value for the } \\
\text { Transfer in million gallon } \\
\text { units; a derived value } \\
\text { representing the product } \\
\text { of the default } \\
\text { RawVolumeValue for the } \\
\text { Transfer and the } \\
\text { CommonConversion } \\
\text { factor to convert to } \\
\text { million gallon common } \\
\text { units. }\end{array}$ \\
\hline 7 & TransferMemo & $\square \square \square$ & Memo & -- & & Note about a Transfer \\
\hline
\end{tabular}




\title{
Table Name: $\quad$ tblVolume
}

Table Description: Volume (quantity of water) recorded for a Transfer based on a specific Determination Method and Unit

Number of Fields: $\quad 9$

Number of Records $\quad 0$

\author{
Dependency: Partial \\ Loading Order: 5
}

Recursive: $\square$

\begin{tabular}{|c|c|c|c|c|c|c|}
\hline & Field Name & PK FK Rqd & Type & Size & Default & Description \\
\hline 1 & Volume_ID & $\square \square \square$ & $\begin{array}{l}\text { AutoNumber } \\
\text { (Long) }\end{array}$ & 4 & & $\begin{array}{l}\text { Key that uniquely } \\
\text { identifies a Volume for a } \\
\text { Transfer }\end{array}$ \\
\hline 2 & Transfer_ID & $\square \nabla \square$ & Long Integer & 4 & & $\begin{array}{l}\text { Key that uniquely } \\
\text { identifies a Transfer }\end{array}$ \\
\hline 3 & Staff_ID & $\square \nabla \square$ & Long Integer & 4 & & $\begin{array}{l}\text { Key that uniquely } \\
\text { identifies a Staff person }\end{array}$ \\
\hline 4 & DataSource_ID & $\square \square \square$ & Long Integer & 4 & & $\begin{array}{l}\text { Key that uniquely } \\
\text { identifies a Data Source }\end{array}$ \\
\hline 5 & VolumeMethod_ID & $\square \nabla \square$ & Long Integer & 4 & & $\begin{array}{l}\text { Key that uniquely } \\
\text { identifies a Method used } \\
\text { to determine the Volume } \\
\text { of a Transfer }\end{array}$ \\
\hline 6 & RawVolumeValue & $\square \square \square$ & Double & 8 & & $\begin{array}{l}\text { Numeric part of the Raw } \\
\text { Volume Value for a } \\
\text { Transfer }\end{array}$ \\
\hline 7 & VolumeUnit_ID & $\square \square \square$ & Long Integer & 4 & & $\begin{array}{l}\text { Key that uniquely } \\
\text { identifies a Volume Unit } \\
\text { (units by which raw } \\
\text { Volume values are } \\
\text { originally recorded or } \\
\text { estimated) for a Transfer }\end{array}$ \\
\hline 8 & IsDefaultVolume & $\square \square \square$ & Yes/No & 1 & Yes & $\begin{array}{l}\text { Identifies a default } \\
\text { Volume to be associated } \\
\text { with a Transfer; 'Yes' } \\
\text { when only one Volume is } \\
\text { associated, or for one } \\
\text { Volume among } \\
\text { alternatives; when 'No', at } \\
\text { least one additional } \\
\text { Volume exists as the } \\
\text { default }\end{array}$ \\
\hline 9 & VolumeMemo & $\square \square \square$ & Memo & -- & & Note about a Volume \\
\hline
\end{tabular}


Appendix 1: Data Dictionary for the New Jersey Water-Transfer Data System (NJWaTr) — Continued

\section{Table Name: $\quad$ tdsAddressType}

Table Description: List of Types of Addresses associated with a Site or an Owner

Number of Fields: 2 Dependency: Independent Recursive: $\square$

Number of Records $3 \quad$ Loading Order: 1

\begin{tabular}{llllll}
\hline \hline Field Name & PK FK Rqd & Type & Size & Default & Description \\
\hline \hline 1 AddressType_ID & $\square \square \square$ & 2 & $\begin{array}{l}\text { Key that uniquely } \\
\text { identifies an Address } \\
\text { Type }\end{array}$ \\
2 AddressType & $\square \square \square$ Text & 20 & $\begin{array}{l}\text { Type of Address (street, } \\
\text { mailing, both) }\end{array}$
\end{tabular}


Appendix 1: Data Dictionary for the New Jersey Water-Transfer Data System (NJWaTr) —Continued

\section{Table Name: $\quad$ tdsConveyanceAction}

Table Description: List of Actions performed by a Conveyance between two Sites

\begin{tabular}{lllll} 
Number of Fields: & 5 & Dependency: & Partial & Recursive: $\square$ \\
Number of Records & 180 & Loading Order: & 2 & \\
\hline
\end{tabular}

\begin{tabular}{|c|c|c|c|c|c|c|}
\hline & Field Name & PK FK Rqd & Type & Size & Default & Description \\
\hline 1 & ConveyanceAction_ID & $\nabla \square \square$ & Integer & 2 & & $\begin{array}{l}\text { Key that uniquely } \\
\text { identifies an Action } \\
\text { performed by a } \\
\text { Conveyance between two } \\
\text { Sites }\end{array}$ \\
\hline 2 & ConveyanceActionCategory_ID & $\square \square \square$ & Integer & 2 & & $\begin{array}{l}\text { Key that uniquely } \\
\text { identifies a Category of } \\
\text { an Action performed by a } \\
\text { Conveyance between two } \\
\text { Sites }\end{array}$ \\
\hline 3 & ConveyanceActionPhrase & $\square \square \square$ & Text & 75 & & $\begin{array}{l}\text { Phrase that describes the } \\
\text { particular Action } \\
\text { performed by a } \\
\text { Conveyance }\end{array}$ \\
\hline 4 & SiteTypeFromID & $\square \square \square$ & Integer & 2 & & $\begin{array}{l}\text { Key that identifies the } \\
\text { SiteType on the 'From' } \\
\text { side of a Conveyance for } \\
\text { a particular Action } \\
\text { between two Sites }\end{array}$ \\
\hline 5 & SiteTypeToID & $\square \square \square$ & Integer & 2 & & $\begin{array}{l}\text { Key that identifies the } \\
\text { SiteType on the 'To' side } \\
\text { of a Conveyance for a } \\
\text { particular Action between } \\
\text { two Sites }\end{array}$ \\
\hline
\end{tabular}


Appendix 1: Data Dictionary for the New Jersey Water-Transfer Data System (NJWaTr) —Continued

\section{Table Name: $\quad$ tdsConveyanceActionCategory}

Table Description: List of Major Categories of Actions performed by a Conveyance between two Sites

Number of Fields: $\quad 2$

Number of Records 25
Dependency: Independent

Loading Order: 1
Recursive:

\begin{tabular}{|c|c|c|c|c|c|c|}
\hline & Field Name & PK FK Rqd & Type & Size & Default & Description \\
\hline 1 & ConveyanceActionCategory_ID & $\nabla \square \square$ & Integer & 2 & & $\begin{array}{l}\text { Key that uniquely } \\
\text { identifies a Category of } \\
\text { an Action performed by a } \\
\text { Conveyance between two } \\
\text { Sites }\end{array}$ \\
\hline 2 & ConveyanceActionCategory & $\square \square \square$ & Text & 50 & & $\begin{array}{l}\text { Term that defines a } \\
\text { Category of an Action } \\
\text { performed by a } \\
\text { Conveyance between two } \\
\text { Sites }\end{array}$ \\
\hline
\end{tabular}


Appendix 1: Data Dictionary for the New Jersey Water-Transfer Data System (NJWaTr) — Continued

\section{Table Name: tdsConveyanceType}

Table Description: $\quad$ List of Types of Conveyances

Number of Fields: $\quad 3$

Number of Records 7

Dependency: Independent Recursive: $\square$

Loading Order: 1

\begin{tabular}{|c|c|c|c|c|c|c|}
\hline & Field Name & PK FK Rqd & Type & Size & Default & Description \\
\hline 1 & ConveyanceType_ID & $\nabla \square \square$ & Integer & 2 & & $\begin{array}{l}\text { Key that uniquely } \\
\text { identifies a Conveyance } \\
\text { Type }\end{array}$ \\
\hline 2 & ConveyanceType & $\square \square \square$ & Text & 20 & & $\begin{array}{l}\text { Type of Conveyance (for } \\
\text { example, pipe, canal, } \\
\text { conduit, and others) }\end{array}$ \\
\hline 3 & ConveyanceTypeMemo & $\square \square \square$ & Memo & -- & & $\begin{array}{l}\text { Note about a } \\
\text { Conveyance Type }\end{array}$ \\
\hline
\end{tabular}


Appendix 1: Data Dictionary for the New Jersey Water-Transfer Data System (NJWaTr) —Continued

\section{Table Name: $\quad$ tdsCounty}

Table Description: List of County Names and Codes

Number of Fields: $8 \quad$ Dependency: Partial Recursive: $\square$

Number of Records $178 \quad$ Loading Order: 2

\begin{tabular}{|c|c|c|c|c|c|c|}
\hline & Field Name & PK FK Rqd & Type & Size & Default & Description \\
\hline 1 & County_ID & $\nabla \square \square$ & Integer & 2 & & $\begin{array}{l}\text { Key that uniquely } \\
\text { identifies a State/County } \\
\text { combination }\end{array}$ \\
\hline 2 & State_ID & $\square \square \square$ & Integer & 2 & & $\begin{array}{l}\text { Key that uniquely } \\
\text { identifies a State or } \\
\text { Canadian Province }\end{array}$ \\
\hline 3 & StateCountyCode & $\square \square \square$ & Text & 5 & & $\begin{array}{l}\text { Concatenation of State } \\
\text { and County Code } \\
\text { numbers (unique) }\end{array}$ \\
\hline 4 & CountyCode & $\square \square \square$ & Text & 3 & & $\begin{array}{l}\text { County Code number } \\
\text { within a State }\end{array}$ \\
\hline 5 & CountyName & $\square \square \square$ & Text & 50 & & County Name \\
\hline 6 & CountyShortName & $\square \square \square$ & Text & 50 & & $\begin{array}{l}\text { County Name without the } \\
\text { word 'County' or 'Parish' }\end{array}$ \\
\hline 7 & CountyLatitude & $\square \square \square$ & Text & 30 & & $\begin{array}{l}\text { Latitude coordinate } \\
\text { (decimal degrees format) } \\
\text { for the centroid of a } \\
\text { County }\end{array}$ \\
\hline 8 & CountyLongitude & $\square \square \square$ & Text & 30 & & $\begin{array}{l}\text { Longitude coordinate } \\
\text { (decimal degrees format) } \\
\text { for the centroid of a } \\
\text { County }\end{array}$ \\
\hline
\end{tabular}


Appendix 1: Data Dictionary for the New Jersey Water-Transfer Data System (NJWaTr) — Continued

\section{Table Name: $\quad$ tdsCriticalArea}

Table Description: List of New Jersey Critical Areas developed for conservation management of regulated aquifers

Number of Fields: 3 Dependency: Independent Recursive: $\square$

Number of Records $4 \quad$ Loading Order: 1

\begin{tabular}{llllll}
\hline \hline Field Name & PK FK Rqd & Type & Size & Default & Description \\
\hline \hline 1 & CriticalArea_ID & $\square \square \square$ & Integer & 2 & $\begin{array}{l}\text { Key that uniquely } \\
\text { identifies a Critical Area }\end{array}$ \\
2 & CriticalAreaCode & $\square \square \square$ & Text & 5 & $\begin{array}{l}\text { Code that uniquely } \\
\text { identifies a Critical Area }\end{array}$ \\
3 & CriticalArea & $\square \square \square$ & Text & 255 & Critical Area description
\end{tabular}


Appendix 1: Data Dictionary for the New Jersey Water-Transfer Data System (NJWaTr) — Continued

\section{Table Name: tdsCriticalZone}

Table Description: List of New Jersey Critical Zone classifications used to identify Sites in threatened or depleted zones of regulated aquifers

Number of Fields: $\quad 3$

Dependency: Independent

Recursive: $\square$

Number of Records 7

Loading Order: 1

\begin{tabular}{llclcl}
\hline \hline Field Name & PK FK Rqd & Type & Size & Default & Description \\
\hline \hline 1 & CriticalZone_ID & $\square \square \square$ & Integer & 2 & $\begin{array}{l}\text { Key that uniquely } \\
\text { identifies a Critical Zone }\end{array}$ \\
2 & CriticalZoneCode & $\square \square \square$ & Text & 5 & $\begin{array}{l}\text { Code that uniquely } \\
\text { identifies a Critical Zone }\end{array}$ \\
3 & CriticalZone & $\square \square \square$ & Text & 255 & Critical Zone description
\end{tabular}


Appendix 1: Data Dictionary for the New Jersey Water-Transfer Data System (NJWaTr) —Continued

\section{Table Name: tdsDroughtRegion}

Table Description: List of New Jersey Drought Regions

Number of Fields: $\quad 3$

Number of Records 7

Dependency: Independent

Recursive:

\begin{tabular}{llclcl}
\hline \hline Field Name & PK FK Rqd & Type & Size & Default & Description \\
\hline \hline 1 DroughtRegion_ID & $\square \square \square$ Integer & 2 & $\begin{array}{l}\text { Key that uniquely } \\
\text { identifies a Drought } \\
\text { Region }\end{array}$ \\
2 DroughtRegionCode & $\square \square \square$ Text & 5 & $\begin{array}{l}\text { Code that uniquely } \\
\text { identifies a Drought } \\
\text { Region }\end{array}$ \\
3 DroughtRegion & $\square \square \square$ Text & 50 & $\begin{array}{l}\text { Drought Region name } \\
\text { D }\end{array}$
\end{tabular}


Appendix 1: Data Dictionary for the New Jersey Water-Transfer Data System (NJWaTr) —Continued

\section{Table Name: $\quad$ tdsHUC}

\section{Table Description: List of Hydrologic Unit Codes (HUCs) and associated information}

Number of Fields: $\quad 9$

Dependency: Partial

Recursive: $\square$

Number of Records 934

Loading Order: 3

\begin{tabular}{|c|c|c|c|c|c|c|}
\hline & Field Name & PK FK Rqd & Type & Size & Default & $\overline{~ D e s c r i p t i o n ~}$ \\
\hline 1 & HUC_ID & $\nabla \square \square$ & Integer & 2 & & $\begin{array}{l}\text { Key that uniquely } \\
\text { identifies a Hydrologic } \\
\text { Unit }\end{array}$ \\
\hline 2 & WaterMgmntArea_ID & $\square \square \square$ & Integer & 2 & & $\begin{array}{l}\text { Key that uniquely } \\
\text { identifies a New Jersey } \\
\text { Water Management Area }\end{array}$ \\
\hline 3 & WatershedCode & $\square \square \square$ & Text & 20 & & $\begin{array}{l}\text { New Jersey Watershed } \\
\text { Code for a 14-digit } \\
\text { hydrologic unit }\end{array}$ \\
\hline 4 & HUC14 & $\square \square \square$ & Text & 14 & & $\begin{array}{l}\text { Hydrologic Unit 14-digit } \\
\text { Code from national list }\end{array}$ \\
\hline 5 & HUC14Name & $\square \square \square$ & Text & 50 & & $\begin{array}{l}\text { Name, if available, of } 14- \\
\text { digit Hydrologic Unit } \\
\text { (USGS names) }\end{array}$ \\
\hline 6 & HUC11 & $\square \square \square$ & Text & 11 & & $\begin{array}{l}\text { Hydrologic Unit 11-digit } \\
\text { Code from national list }\end{array}$ \\
\hline 7 & HUC11Name & $\square \square \square$ & Text & 50 & & $\begin{array}{l}\text { Name, if available, of } 11- \\
\text { digit Hydrologic Unit } \\
\text { (NJDEP names) }\end{array}$ \\
\hline 8 & HUC8 & $\square \square \square$ & Text & 8 & & $\begin{array}{l}\text { Hydrologic Unit 8-digit } \\
\text { Code from national list }\end{array}$ \\
\hline 9 & HUC8Name & $\square \square \square$ & Text & 50 & & $\begin{array}{l}\text { Name, if available, of 8- } \\
\text { digit Hydrologic Unit } \\
\text { (USGS names) }\end{array}$ \\
\hline
\end{tabular}


Appendix 1: Data Dictionary for the New Jersey Water-Transfer Data System (NJWaTr) —Continued

\section{Table Name: $\quad$ tdsLocationScale}

Table Description: $\quad$ List of Scale terms (point, MCD, County, and others) that describe the spatial extent of a Location

Number of Fields: 2 Dependency: Independent Recursive: $\square$

Number of Records $8 \quad$ Loading Order: 1

\begin{tabular}{|c|c|c|c|c|c|c|}
\hline & Field Name & PK FK Rqd & Type & $\overline{\text { Size }}$ & $\overline{\text { Default }}$ & Description \\
\hline 1 & LocationScale_ID & $\nabla \square \square$ & Integer & 2 & & $\begin{array}{l}\text { Key that uniquely } \\
\text { identifies a spatial Scale } \\
\text { term for a Location }\end{array}$ \\
\hline 2 & LocationScale & $\square \square \square$ & Text & 50 & & $\begin{array}{l}\text { Term that describes the } \\
\text { spatial Scale of a } \\
\text { Location (for example, } \\
\text { point, MCD, County, } \\
\text { State, HUC, and others) }\end{array}$ \\
\hline
\end{tabular}




\section{Table Name: $\quad$ tdsMCD}

\section{Table Description: List of Minor Civil Divisions (MCDs or Subcounty divisions) of the US Census Bureau}

Number of Fields: $\quad 10$

Number of Records $\quad 4,479$

$$
\begin{array}{ll}
\text { Dependency: } & \text { Partial } \\
\text { Loading Order: } & 3
\end{array}
$$

\begin{tabular}{|c|c|c|c|c|c|c|}
\hline & Field Name & PK FK Rqd & Type & Size & Default & Description \\
\hline 1 & MCD_ID & $\square \square \square$ & Integer & 2 & & $\begin{array}{l}\text { Key that uniquely } \\
\text { identifies a MCD (Minor } \\
\text { Civil Division of US } \\
\text { Census Bureau) }\end{array}$ \\
\hline 2 & County_ID & $\square \square \square$ & Integer & 2 & & $\begin{array}{l}\text { Key that uniquely } \\
\text { identifies a State/County } \\
\text { combination }\end{array}$ \\
\hline 3 & DroughtRegion_ID & $\square \square \square$ & Integer & 2 & & $\begin{array}{l}\text { Key that uniquely } \\
\text { identifies a Drought } \\
\text { Region }\end{array}$ \\
\hline 4 & StateMCDCode & $\square \square \square$ & Text & 7 & & $\begin{array}{l}\text { Concatenation of State } \\
\text { and MCD Code numbers } \\
\text { (unique within a County) }\end{array}$ \\
\hline 5 & MCDCode & $\square \square \square$ & Text & 5 & & MCD Code within a State \\
\hline 6 & MCDType & $\square \square \square$ & Text & 20 & & $\begin{array}{l}\text { Type of MCD (for } \\
\text { example, town, city, } \\
\text { plantation, reservation, } \\
\text { and others) }\end{array}$ \\
\hline 7 & MCDName & $\square \square \square$ & Text & 50 & & MCD Name \\
\hline 8 & MCDShortName & $\square \square \square$ & Text & 50 & & $\begin{array}{l}\text { Short name for MCD } \\
\text { (excludes MCD type, } \\
\text { which is part of formal } \\
\text { Census MCD name) }\end{array}$ \\
\hline 9 & MCDLatitude & $\square \square \square$ & Text & 30 & & $\begin{array}{l}\text { Latitude coordinate } \\
\text { (decimal degrees format) } \\
\text { for the centroid of a MCD }\end{array}$ \\
\hline 10 & MCDLongitude & $\square \square \square$ & Text & 30 & & $\begin{array}{l}\text { Longitude coordinate } \\
\text { (decimal degrees format) } \\
\text { for the centroid of a MCD }\end{array}$ \\
\hline
\end{tabular}

Recursive: $\square$ 
Appendix 1: Data Dictionary for the New Jersey Water-Transfer Data System (NJWaTr) — Continued

\section{Table Name: tdsOwnerType}

\section{Table Description: $\quad$ List of Types of Owners}

Number of Fields: $\quad 3$

Number of Records 7

Dependency: Independent Recursive: $\square$

Loading Order: 1

\begin{tabular}{llllll}
\hline \hline Field Name & PK FK Rqd & Type & Size & Default & Description \\
\hline \hline $1 \quad$ OwnerType_ID & $\square \square \square$ Integer & 2 & $\begin{array}{l}\text { Key that uniquely } \\
\text { identifies an Owner Type }\end{array}$ \\
2 OwnerType & $\square \square \square \quad$ Text & 25 & $\begin{array}{l}\text { Type of Owner (for } \\
\text { example, federal, state, } \\
\text { municipal, private, and } \\
\text { others) }\end{array}$ \\
3 & OwnerTypeMemo & & $\begin{array}{l}\text { Note about an Owner } \\
\text { Type }\end{array}$
\end{tabular}


Appendix 1: Data Dictionary for the New Jersey Water-Transfer Data System (NJWaTr) — Continued

\section{Table Name: $\quad$ tdsPermitSeries}

Table Description: $\quad$ List of Series groupings for Permits

Number of Fields: 3 Dependency: Independent Recursive: $\square$

Number of Records $10 \quad$ Loading Order: 1

\begin{tabular}{llclcl}
\hline \hline Field Name & PK FK Rqd & Type & Size & Default & Description \\
\hline \hline 1 & PermitSeries_ID & $\square \square \square$ & Integer & 2 & $\begin{array}{l}\text { Key that uniquely } \\
\text { identifies a Permit Series }\end{array}$ \\
2 & PermitSeries & $\square \square \square$ & Text & 50 & $\begin{array}{l}\text { Series grouping for } \\
\text { Permits }\end{array}$ \\
3 & PermitSeriesDescription & $\square \square \square$ & Text & 120 & $\begin{array}{l}\text { Description of a Series } \\
\text { grouping for Permits }\end{array}$
\end{tabular}


Appendix 1: Data Dictionary for the New Jersey Water-Transfer Data System (NJWaTr) —Continued

\section{Table Name: $\quad$ tdsResourceType}

Table Description: $\quad$ List of Types of water Resources

Number of Fields: $\quad 4$

Dependency: Independent

Recursive: $\square$

Number of Records 7

Loading Order: 1

\begin{tabular}{llllll}
\hline \hline Field Name & PK FK Rqd & Type & Size & Default & Description \\
\hline \hline $1 \quad$ ResourceType_ID & $\square \square \square$ & Integer & 2 & $\begin{array}{l}\text { Key that uniquely } \\
\text { identifies a water } \\
\text { Resource Type } \\
\text { GW (ground water) or }\end{array}$ \\
& $\square \square \square$ & Text & 2 & $\begin{array}{l}\text { GW (surface water) } \\
\text { identifier for a }\end{array}$ \\
3 Salinity & $\square \square \square$ & Text & & $\begin{array}{l}\text { ResourceType } \\
\text { Salinity group of a }\end{array}$ \\
& & & 2 & $\begin{array}{l}\text { ResourceType (fresh, } \\
\text { brackish or saline) }\end{array}$ \\
4 ResourceType & $\square \square$ & Text & & $\begin{array}{l}\text { Type of water Resource } \\
\text { (for example, fresh } \\
\text { ground-water, brackish } \\
\text { surface-water, and others) }\end{array}$
\end{tabular}


Appendix 1: Data Dictionary for the New Jersey Water-Transfer Data System (NJWaTr) — Continued

\section{Table Name: tdsSiteType}

Table Description: $\quad$ List of Types of Sites

Number of Fields: $\quad 4$

Dependency: Partial

Recursive: $\square$

Number of Records 36

Loading Order: 3

\begin{tabular}{|c|c|c|c|c|c|c|}
\hline & Field Name & PK FK Rqd & Type & Size & Default & Description \\
\hline 1 & SiteType_ID & $\nabla \square \square$ & Integer & 2 & & $\begin{array}{l}\text { Key that uniquely } \\
\text { identifies a Site Type }\end{array}$ \\
\hline 2 & SiteTypeSubcategory_ID & $\square \square \square$ & Integer & 2 & & $\begin{array}{l}\text { Key that uniquely } \\
\text { identifies a Subcategory } \\
\text { for describing Site Types }\end{array}$ \\
\hline 3 & SiteType & $\square \square \square$ & Text & 50 & & $\begin{array}{l}\text { Type of Site (for example, } \\
\text { well, discharge pipe, } \\
\text { regional distribution Site, } \\
\text { potable treatment plant, } \\
\text { single user, and others) }\end{array}$ \\
\hline 4 & SiteTypeMemo & $\square \square \square$ & Memo & -- & & Note about a Site Type \\
\hline
\end{tabular}


Appendix 1: Data Dictionary for the New Jersey Water-Transfer Data System (NJWaTr) — Continued

\section{Table Name: $\quad$ tdsSiteTypeCategory}

Table Description: List of Major Categories for describing Types of Sites

Number of Fields: $\quad 2$

Dependency: Independent

Recursive: $\square$

Number of Records 7

Loading Order: 1

\begin{tabular}{|c|c|c|c|c|c|c|}
\hline & Field Name & PK FK Rqd & Type & Size & Default & Description \\
\hline 1 & SiteTypeCategory_ID & $\nabla \square \square$ & Integer & 2 & & $\begin{array}{l}\text { Key that uniquely } \\
\text { identifies a major } \\
\text { Category for describing } \\
\text { Site Types }\end{array}$ \\
\hline 2 & SiteTypeCategory & $\square \square \square$ & Text & 25 & & $\begin{array}{l}\text { Term that defines a major } \\
\text { Category of Site Types }\end{array}$ \\
\hline
\end{tabular}


Appendix 1: Data Dictionary for the New Jersey Water-Transfer Data System (NJWaTr) —Continued

\section{Table Name: $\quad$ tdsSiteTypeSubcategory}

Table Description: $\quad$ List of Subcategories for describing Types of Sites, with link to Major Categories

Number of Fields: $\quad 3$

Number of Records 13

$\begin{array}{ll}\text { Dependency: } & \text { Partial } \\ \text { Loading Order: } & 2\end{array}$

Recursive: $\square$

\begin{tabular}{llllll}
\hline \hline Field Name & PK FK Rqd & Type & Size & Default & Description \\
\hline \hline 1 SiteTypeSubcategory_ID & $\square \square \square$ & Integer & 2 & $\begin{array}{l}\text { Key that uniquely } \\
\text { identifies a Subcategory } \\
\text { for describing Site Types }\end{array}$ \\
2 SiteTypeCategory_ID & $\square \square \square$ & Integer & 2 & $\begin{array}{l}\text { Key that uniquely } \\
\text { identifies a major } \\
\text { Category for describing } \\
\text { Site Types }\end{array}$ \\
3 SiteTypeSubcategory & $\square \square \square$ & Text & 25 & $\begin{array}{l}\text { Term that defines a } \\
\text { Subcategory of Site } \\
\text { Types }\end{array}$
\end{tabular}


Appendix 1: Data Dictionary for the New Jersey Water-Transfer Data System (NJWaTr) —Continued

\section{Table Name: $\quad$ tdsState}

Table Description: List of States and State Codes

Number of Fields: 7

Number of Records 6

Dependency: Independent Recursive: $\square$

Loading Order: 1

\begin{tabular}{|c|c|c|c|c|c|c|}
\hline & Field Name & PK FK Rqd & Type & Size & Default & Description \\
\hline 1 & State_ID & $\nabla \square \nabla$ & Integer & 2 & & $\begin{array}{l}\text { Key that uniquely } \\
\text { identifies a State or } \\
\text { Canadian Province }\end{array}$ \\
\hline 2 & CountryAbbrv & $\square \square \square$ & Text & 3 & "USA" & $\begin{array}{l}\text { Country Abbreviation } \\
\text { (USA or CAN) }\end{array}$ \\
\hline 3 & StateCode & $\square \square \square$ & Text & 2 & & $\begin{array}{l}\text { FIPS State Code, or } \\
\text { surrogate for Canadian } \\
\text { Province }\end{array}$ \\
\hline 4 & StateAbbrv & $\square \square \square$ & Text & 2 & & $\begin{array}{l}\text { State (or Canadian } \\
\text { Province) Abbreviation }\end{array}$ \\
\hline 5 & StateName & $\square \square \square$ & Text & 50 & & $\begin{array}{l}\text { State (or Canadian } \\
\text { Province) Name }\end{array}$ \\
\hline 6 & StateLatitude & $\square \square \square$ & Text & 30 & & $\begin{array}{l}\text { Latitude coordinate } \\
\text { (decimal degrees format) } \\
\text { for the centroid of a State }\end{array}$ \\
\hline 7 & StateLongitude & $\square \square \square$ & Text & 30 & & $\begin{array}{l}\text { Longitude coordinate } \\
\text { (decimal degrees format) } \\
\text { for the centroid of a State }\end{array}$ \\
\hline
\end{tabular}


Appendix 1: Data Dictionary for the New Jersey Water-Transfer Data System (NJWaTr) — Continued

\section{Table Name: $\quad$ tdsTimeInterval}

Table Description: List of Time intervals (year, month, week, day, multi-day) over which Transfers and Site Details apply

Number of Fields: $\quad 2$

Number of Records 11

Dependency: Independent

Recursive:

(2)

Loading Order: 1

\begin{tabular}{llllll}
\hline \hline Field Name & PK FK Rqd & Type & Size & Default & Description \\
\hline \hline 1 & TimeInterval_ID & $\square \square \square$ & Integer & 2 & $\begin{array}{l}\text { Key that uniquely } \\
\text { identifies a Time Interval }\end{array}$ \\
2 TimeInterval & $\square \square \square$ & Text & 25 & $\begin{array}{l}\text { Term that defines a Time } \\
\text { Interval (for example, } \\
\text { year, month, week, day, } \\
\text { and others) }\end{array}$
\end{tabular}


Appendix 1: Data Dictionary for the New Jersey Water-Transfer Data System (NJWaTr) — Continued

\section{Table Name: $\quad$ tds UseGroup}

Table Description: $\quad$ List of major Groupings for New Jersey Water Use Types that classify Sites

Number of Fields: $\quad 3$

Dependency: Independent

Recursive: $\square$

Number of Records 8

Loading Order: 1

\begin{tabular}{llllll}
\hline \hline Field Name & PK FK Rqd & Type & Size & Default & Description \\
\hline \hline 1 & UseGroup_ID & $\square \square \square$ & Integer & 2 & $\begin{array}{l}\text { Key that uniquely } \\
\text { identifies a Water Use } \\
\text { Group classification for a } \\
\text { Site }\end{array}$ \\
& & & & Water Use Group Code \\
2 & UseGroupCode & $\square \square \square$ & Text & 2 & $\begin{array}{l}\text { Water Use Group Name } \\
\text { or short description }\end{array}$ \\
3 & UseGroup & $\square \square \square$ & Text & 50 &
\end{tabular}


Appendix 1: Data Dictionary for the New Jersey Water-Transfer Data System (NJWaTr) —Continued

\section{Table Name: tdsUseType}

Table Description: List of New Jersey Water Use Types that classify Sites

Number of Fields: $\quad 4$

Dependency: Partial

Recursive: $\square$

Number of Records 38

Loading Order: 2

\begin{tabular}{|c|c|c|c|c|c|c|}
\hline & Field Name & PK FK Rqd & Type & Size & Default & Description \\
\hline 1 & UseType_ID & $\nabla \square \square$ & Integer & 2 & & $\begin{array}{l}\text { Key that uniquely } \\
\text { identifies a Water Use } \\
\text { Type classification for a } \\
\text { Site }\end{array}$ \\
\hline 2 & UseGroup_ID & $\square \square \square$ & Integer & 2 & & $\begin{array}{l}\text { Key that uniquely } \\
\text { identifies a Water Use } \\
\text { Group classification for a } \\
\text { Site }\end{array}$ \\
\hline 3 & UseTypeCode & $\square \square \square$ & Text & 2 & & $\begin{array}{l}\text { Water Use Type Code } \\
\text { within a Use Group }\end{array}$ \\
\hline 4 & UseType & $\square \square \square$ & Text & 50 & & $\begin{array}{l}\text { Water Use Type Code } \\
\text { Name }\end{array}$ \\
\hline
\end{tabular}




\section{Table Name: $\quad$ tds VolumeUnitDecimal}

Table Description: List of choices for the Decimal part of a VolumeUnit (ten, hundred, thousand, and others) and their conversion factor to Million to get a common Volume unit of 'million gallons'

Number of Fields: $\quad 3$

Dependency: Independent

Recursive:

Number of Records 10

Loading Order: 1

\begin{tabular}{|c|c|c|c|c|c|c|}
\hline & Field Name & PK FK Rqd & Type & Size & Default & Description \\
\hline 1 & VolumeUnitDecimal_ID & $\nabla \square \square$ & Integer & 2 & & $\begin{array}{l}\text { Key that uniquely } \\
\text { identifies a Volume Unit } \\
\text { Decimal component }\end{array}$ \\
\hline 2 & DecimalUnit & $\square \square \square$ & Text & 50 & & $\begin{array}{l}\text { Term used to describe the } \\
\text { Decimal part of a Volume } \\
\text { Unit (for example, } \\
\text { thousand, million) }\end{array}$ \\
\hline 3 & ConversionToMillion & $\square \square \square$ & Double & 8 & & $\begin{array}{l}\text { Factor used to convert } \\
\text { Decimal Unit to one } \\
\text { equivalent in Millions }\end{array}$ \\
\hline
\end{tabular}




\section{Table Name: $\quad$ tdsVolumeUnitQuantity}

Table Description: List of choices for the Quantity part of a VolumeUnit (gallon, cubic feet, acre-feet, and others) and their conversion factor to Gallon to get a common Quantity unit of 'million gallons'

Number of Fields: $\quad 3$

Dependency: Independent

Recursive:

Number of Records 6

Loading Order: 1

\begin{tabular}{|c|c|c|c|c|c|c|}
\hline & Field Name & PK FK Rqd & Type & Size & Default & Description \\
\hline 1 & VolumeUnitQuantity_ID & $\square \square \square$ & Integer & 2 & & $\begin{array}{l}\text { Key that uniquely } \\
\text { identifies a Volume Unit } \\
\text { Quantity component }\end{array}$ \\
\hline 2 & QuantityUnit & $\square \square \square$ & Text & 50 & & $\begin{array}{l}\text { Term used to describe the } \\
\text { Quantity part of a Volume } \\
\text { Unit (for example, gallons, } \\
\text { cubic feet, acre-feet) }\end{array}$ \\
\hline 3 & ConversionToGallon & $\square \square \square$ & Double & 8 & & $\begin{array}{l}\text { Factor used to convert } \\
\text { Quantity Unit to one } \\
\text { equivalent in Gallons }\end{array}$ \\
\hline
\end{tabular}


Appendix 1: Data Dictionary for the New Jersey Water-Transfer Data System (NJWaTr) —Continued

\section{Table Name: $\quad$ tdsWaterBodyType}

Table Description: $\quad$ List of Types of Water Bodies represented by a water Resource, such as pond/lake, reservoir, river or estuary

Number of Fields: $\quad 3$

Number of Records 14

Dependency: Partial

Loading Order: 2

Recursive: $\square$

\begin{tabular}{|c|c|c|c|c|c|c|}
\hline & Field Name & PK FK Rqd & Type & Size & Default & Description \\
\hline 1 & WaterBodyType_ID & $\nabla \square \square$ & Integer & 2 & & $\begin{array}{l}\text { Key that uniquely } \\
\text { identifies a Type of } \\
\text { Water Body represented } \\
\text { by a water Resource (for } \\
\text { example, lake, river, } \\
\text { estuary) }\end{array}$ \\
\hline 2 & ResourceType_ID & $\square \square \square$ & Integer & 2 & & $\begin{array}{l}\text { Key that uniquely } \\
\text { identifies a water } \\
\text { Resource Type }\end{array}$ \\
\hline 3 & WaterBodyType & $\square \square \square$ & Text & 30 & & $\begin{array}{l}\text { Type of Water Body } \\
\text { describing a water } \\
\text { Resource (for example, } \\
\text { river, lake, estuary, ocean, } \\
\text { and others) }\end{array}$ \\
\hline
\end{tabular}


Appendix 1: Data Dictionary for the New Jersey Water-Transfer Data System (NJWaTr) —Continued

\section{Table Name: $\quad$ tdsWaterMgmntArea}

Table Description: List of New Jersey Water Management Areas

Number of Fields: 4 Dependency: Partial Recursive: $\square$

Number of Records $21 \quad$ Loading Order: 2

\begin{tabular}{llclcl}
\hline \hline Field Name & PK FK Rqd & Type & Size & Default & Description \\
\hline \hline $1 \quad$ WaterMgmntArea_ID & $\square \square \square$ & 2 & $\begin{array}{l}\text { Key that uniquely } \\
\text { identifies a New Jersey } \\
\text { Water Management Area }\end{array}$ \\
2 WaterRegion_ID & $\square \square \square$ Integer & Integer & 2 & $\begin{array}{l}\text { Key that uniquely } \\
\text { identifies a New Jersey } \\
\text { Water Region }\end{array}$ \\
3 WaterMgmntAreaCode & $\square \square \square$ & Text & 50 & $\begin{array}{l}\text { Two-digit Code used to } \\
\text { uniquely identify a New } \\
\text { Jersey Water } \\
\text { Management Area }\end{array}$ \\
& & & & & $\begin{array}{l}\text { Name of New Jersey } \\
\text { Water Management Area }\end{array}$
\end{tabular}


Appendix 1: Data Dictionary for the New Jersey Water-Transfer Data System (NJWaTr) —Continued

\section{Table Name: $\quad$ tdsWaterRegion}

Table Description: List of New Jersey Water Regions

Number of Fields: 3 Dependency: Independent Recursive: $\square$

Number of Records $6 \quad$ Loading Order: 1

\begin{tabular}{llllll}
\hline \hline Field Name & PK FK Rqd & Type & Size & Default & Description \\
\hline \hline $1 \quad$ WaterRegion_ID & $\square \square \square$ & Integer & 2 & $\begin{array}{l}\text { Key that uniquely } \\
\text { identifies a New Jersey } \\
\text { Water Region }\end{array}$ \\
2 WaterRegionCode & $\square \square \square$ & Text & 50 & $\begin{array}{l}\text { Code used to uniquely } \\
\text { identify a New Jersey } \\
\text { Water Region }\end{array}$ \\
3 WaterRegion & $\square \square \square$ & Text & 50 & $\begin{array}{l}\text { Name of New Jersey } \\
\text { Water Region }\end{array}$
\end{tabular}


Appendix 1: Data Dictionary for the New Jersey Water-Transfer Data System (NJWaTr) — Continued

\section{Table Name: $\quad$ tdxAliasLabel}

Table Description: User-Extendable List of standardized Labels used for Alias assignments

Number of Fields: 5 Dependency: Partial Recursive: $\square$

Number of Records $\quad 0 \quad$ Loading Order: 4

\begin{tabular}{llllll}
\hline \hline Field Name & PK FK Rqd & Type & Size & Default & Description \\
\hline \hline 1 & AliasLabel_ID & $\square \square \square$ & $\begin{array}{l}\text { AutoNumber } \\
\text { (Long) }\end{array}$ & 4 & $\begin{array}{l}\text { Key that uniquely } \\
\text { identifies an AliasLabel }\end{array}$ \\
2 DataSource_ID & $\square \square \square$ & Long Integer & 4 & $\begin{array}{l}\text { Key that uniquely } \\
\text { identifies a Data Source }\end{array}$ \\
3 & AliasSource & $\square \square \square$ & Text & 75 & $\begin{array}{l}\text { Source of an Alias; } \\
\text { optional, provides more } \\
\text { detail within a DataSource }\end{array}$ \\
4 & AliasLabel & $\square \square \square$ & Text & 50 & $\begin{array}{l}\text { Label for an Alias, for } \\
\text { example StationID }\end{array}$ \\
5 & AliasLabelMemo & $\square \square \square$ & Memo & -- & Note about an Alias Label
\end{tabular}


Appendix 1: Data Dictionary for the New Jersey Water-Transfer Data System (NJWaTr) —Continued

\section{Table Name: $\quad$ tdxConveyanceDetailLabel}

Table Description: User-Extendable List of standardized Labels used for Conveyance Details

Number of Fields: $\quad 3$

Dependency: Independent

Recursive: $\square$

Number of Records 5

Loading Order: 1

\begin{tabular}{llllcl}
\hline \hline Field Name & PK FK Rqd & Type & Size & Default & Description \\
\hline \hline 1 & ConveyanceDetailLabel_ID & $\square \square \square$ & $\begin{array}{l}\text { AutoNumber } \\
\text { (Long) }\end{array}$ & 4 & $\begin{array}{l}\text { Key that uniquely } \\
\text { identifies a Conveyance } \\
\text { Detail Label }\end{array}$ \\
2 ConveyanceDetailLabel & $\square \square \square$ & Text & 50 & $\begin{array}{l}\text { Label describing the } \\
\text { numeric or textual value } \\
\text { of a Conveyance Detail } \\
3\end{array}$ ConveyanceDetailLabelMemo \\
$\square \square \square$ & Memo & -- & $\begin{array}{l}\text { Note about a } \\
\text { Conveyance Detail Label }\end{array}$
\end{tabular}


Appendix 1: Data Dictionary for the New Jersey Water-Transfer Data System (NJWaTr) — Continued

\section{Table Name: $\quad$ tdxDataSource}

Table Description: User-Extendable List of the Sources of Data being stored

Number of Fields: 4

Dependency: Partial

Recursive: $\square$

Number of Records 4

Loading Order: 3

\begin{tabular}{llllcl}
\hline \hline Field Name & PK FK Rqd & Type & Size & Default & Description \\
\hline \hline 1 & DataSource_ID & $\square \square \square$ & $\begin{array}{l}\text { AutoNumber } \\
\text { (Long) }\end{array}$ & 4 & $\begin{array}{l}\text { Key that uniquely } \\
\text { identifies a Data Source }\end{array}$ \\
2 & Owner_ID & $\square \square \square$ & Long Integer & 4 & $\begin{array}{l}\text { Key that uniquely } \\
\text { identifies an Owner }\end{array}$ \\
3 & DataSource & $\square \square \square$ & Text & 75 & Data Source name \\
4 & DataSourceMemo & $\square \square \square$ & Memo & -- & Note about a Data Source
\end{tabular}


Appendix 1: Data Dictionary for the New Jersey Water-Transfer Data System (NJWaTr) — Continued

\section{Table Name: $\quad$ tdxLocationDetMethod}

Table Description: User-Extendable List of Location Determination Methods for Sites

Number of Fields: $\quad 2$

Number of Records 11

Dependency: Independent

Recursive: $\square$

Preld Name PK FK Rqd Type

\begin{tabular}{llllll}
\hline \hline Field Name & PK FK Rqd & Type & Size & Default & Description \\
\hline \hline 1 & LocationDetMethod_ID & $\square \square \square$ & $\begin{array}{l}\text { AutoNumber } \\
\text { (Long) }\end{array}$ & 4 & $\begin{array}{l}\text { Key that uniquely } \\
\text { identifies a Location } \\
\text { Determination Method }\end{array}$ \\
2 & LocationDetMethod & $\square \square \square$ & Text & 50 & $\begin{array}{l}\text { Location Determination } \\
\text { Method }\end{array}$
\end{tabular}


Appendix 1: Data Dictionary for the New Jersey Water-Transfer Data System (NJWaTr) — Continued

\section{Table Name: $\quad$ tdxPermitLabel}

Table Description: User-Extendable List of standardized Labels used to identify different Permits

Number of Fields: $\quad 4$

Number of Records 3

Dependency: Partial

Loading Order: 4

Recursive: $\square$

\begin{tabular}{llllll}
\hline \hline Field Name & PK FK Rqd & Type & Size & Default & Description \\
\hline \hline 1 & PermitLabel_ID & $\square \square \square$ & $\begin{array}{l}\text { AutoNumber } \\
\text { (Long) }\end{array}$ & 4 & $\begin{array}{l}\text { Key that uniquely } \\
\text { identifies a Permit Label }\end{array}$ \\
2 & DataSource_ID & $\square \square \square$ & Long Integer & 4 & $\begin{array}{l}\text { Key that uniquely } \\
\text { identifies a Data Source } \\
3\end{array}$ \\
$\begin{array}{l}\text { PermitLabel } \\
\end{array}$ & $\square \square$ & Text & 50 & $\begin{array}{l}\text { Label identifying a } \\
\text { specific type of Permit } \\
\text { for example, PWSID, } \\
\text { BWA, NJDPES) }\end{array}$ \\
4 & PermitLabelMemo & $\square \square \square$ & Memo & -- & Note about a Permit Label
\end{tabular}


Appendix 1: Data Dictionary for the New Jersey Water-Transfer Data System (NJWaTr) — Continued

\section{Table Name: $\quad$ tdxResourceDetailLabel}

Table Description: User-Extendable List of standardized Labels used for Resource Details

Number of Fields: $\quad 3$

Dependency: Independent

Recursive: $\square$

Number of Records 5

Loading Order: 1

\begin{tabular}{llllll}
\hline \hline Field Name & PK FK Rqd & Type & Size & Default & Description \\
\hline \hline 1 & ResourceDetailLabel_ID & $\square \square \square$ & $\begin{array}{l}\text { AutoNumber } \\
\text { (Long) }\end{array}$ & 4 & $\begin{array}{l}\text { Key that uniquely } \\
\text { identifies a Resource } \\
\text { Detail Label }\end{array}$ \\
2 & ResourceDetailLabel & $\square \square \square$ & Text & 50 & $\begin{array}{l}\text { Label describing the } \\
\text { numeric or textual value } \\
\text { of a Resource Detail }\end{array}$ \\
3 & ResourceDetailLabelMemo & $\square \square \square$ & Memo & - & $\begin{array}{l}\text { Note about a Resource } \\
\text { Detail Label }\end{array}$
\end{tabular}


Appendix 1: Data Dictionary for the New Jersey Water-Transfer Data System (NJWaTr) —Continued

\section{Table Name: $\quad$ tdxSiteDetailCategory}

Table Description: User-Extendable List of Categories used for time-dependent Site Details

Number of Fields: 2

Dependency: Independent

Recursive: $\square$

Number of Records 5

Loading Order: 1

\begin{tabular}{llllll}
\hline \hline Field Name & PK FK Rqd & Type & Size & Default & Description \\
\hline \hline 1 & SiteDetailCategory_ID & $\square \square \square$ & $\begin{array}{l}\text { AutoNumber } \\
\text { (Long) }\end{array}$ & 4 & $\begin{array}{l}\text { Key that uniquely } \\
\text { identifies a Category for } \\
\text { describing Details about } \\
\text { a Site }\end{array}$ \\
2 SiteDetailCategory & $\square \square \square$ Text & 50 & $\begin{array}{l}\text { Term that defines a } \\
\text { Category for describing } \\
\text { Details about a Site }\end{array}$
\end{tabular}


Appendix 1: Data Dictionary for the New Jersey Water-Transfer Data System (NJWaTr) —Continued

\section{Table Name: $\quad$ tdxSiteDetailLabel}

Table Description: User-Extendable List of Labels used for time-dependent Site Details

Number of Fields: 6

Dependency: Partial

Recursive:

Number of Records 15

Loading Order: 2

\begin{tabular}{|c|c|c|c|c|c|c|}
\hline & Field Name & PK FK Rqd & Type & Size & Default & Description \\
\hline 1 & SiteDetailLabel_ID & $\nabla \square \nabla$ & $\begin{array}{l}\text { AutoNumber } \\
\text { (Long) }\end{array}$ & 4 & & $\begin{array}{l}\text { Key that uniquely } \\
\text { identifies a Site Detail } \\
\text { Label }\end{array}$ \\
\hline 2 & SiteDetailCategory_ID & $\square \square \square$ & Long Integer & 4 & & $\begin{array}{l}\text { Key that uniquely } \\
\text { identifies a Category for } \\
\text { describing Details about } \\
\text { a Site }\end{array}$ \\
\hline 3 & SiteDetailLabel & $\square \square \square$ & Text & 50 & & $\begin{array}{l}\text { Label describing the } \\
\text { numeric or textual value } \\
\text { of a Site Detail }\end{array}$ \\
\hline 4 & IsNumericDetail & $\square \square \square$ & Yes/No & 1 & & $\begin{array}{l}\text { Identifies a Site Detail } \\
\text { Label for numeric values; } \\
\text { 'Yes' only for labels } \\
\text { associated with numeric } \\
\text { values }\end{array}$ \\
\hline 5 & SiteDetailUnit & $\square \square \square$ & Text & 50 & & $\begin{array}{l}\text { Optional Unit for a Site } \\
\text { Detail Label, but Required } \\
\text { for labels representing } \\
\text { numeric values }\end{array}$ \\
\hline 6 & SiteDetailLabelMemo & $\square \square \square$ & Memo & -- & & $\begin{array}{l}\text { Note about a Site Detail } \\
\text { Label }\end{array}$ \\
\hline
\end{tabular}


Appendix 1: Data Dictionary for the New Jersey Water-Transfer Data System (NJWaTr) —Continued

\section{Table Name: $\quad$ tdxStaff}

Table Description: $\quad$ User-Extendable List of Staff entering, reviewing or evaluating Transfer Volume data

Number of Fields: $\quad 5$

Number of Records 1

$$
\begin{aligned}
& \text { Dependency: Independent Recursive: } \square \\
& \text { Loading Order: } 1
\end{aligned}
$$

\begin{tabular}{llllll}
\hline \hline Field Name & PK FK Rqd & Type & Size & Default & Description \\
\hline \hline 1 & Staff_ID & $\square \square \square$ & $\begin{array}{l}\text { AutoNumber } \\
\text { (Long) }\end{array}$ & 4 & $\begin{array}{l}\text { Key that uniquely } \\
\text { identifies a Staff person } \\
2\end{array}$ \\
$\begin{array}{llll}\text { StaffInitials } \\
\text { (unique code for each }\end{array}$ \\
3 StaffName & $\square \square \square$ & Text & 4 & $\begin{array}{l}\text { individual) } \\
\text { Text }\end{array}$ \\
4 & StaffAffiliation & 50 & $\begin{array}{l}\text { (format: last name, first } \\
\text { name, initial) }\end{array}$ \\
5 & StaffMemo & $\square \square \square$ & Text & 50 & Affiliation of Staff person \\
& $\square \square \square$ & Memo & -- & Note about Staff person
\end{tabular}


Appendix 1: Data Dictionary for the New Jersey Water-Transfer Data System (NJWaTr) —Continued

\section{Table Name: $\quad$ tdxSystem}

Table Description: User-Extendable List of Systems consisting of two or more associated Sites

Number of Fields: $5 \quad$ Dependency: Partial Recursive: $\square$

Number of Records $0 \quad$ Loading Order: 2

\begin{tabular}{|c|c|c|c|c|c|c|}
\hline & Field Name & PK FK Rqd & Type & Size & Default & Description \\
\hline 1 & System_ID & $\square \square \square$ & $\begin{array}{l}\text { AutoNumber } \\
\text { (Long) }\end{array}$ & 4 & & $\begin{array}{l}\text { Key that uniquely } \\
\text { identifies a System }\end{array}$ \\
\hline 2 & SystemType_ID & $\square \square \square$ & Long Integer & 4 & & $\begin{array}{l}\text { Key that uniquely } \\
\text { identifies a Type of } \\
\text { System }\end{array}$ \\
\hline 3 & SystemName & $\square \square \square$ & Text & 200 & & $\begin{array}{l}\text { Name of a System } \\
\text { consisting of one or more } \\
\text { Sites }\end{array}$ \\
\hline 4 & ParentSystem_ID & $\square \square \square$ & Long Integer & 4 & & $\begin{array}{l}\text { Key that uniquely } \\
\text { identifies the Parent } \\
\text { System of the current } \\
\text { System (accommodates } \\
\text { nested Systems) }\end{array}$ \\
\hline 5 & SystemMemo & $\square \square \square$ & Memo & -- & & Note about a System \\
\hline
\end{tabular}


Appendix 1: Data Dictionary for the New Jersey Water-Transfer Data System (NJWaTr) —Continued

\section{Table Name: tdxSystemType}

Table Description: $\quad$ User-Extendable List of Types of Systems (aggregates of Sites)

Number of Fields: 3

Dependency: Independent

Recursive: $\square$

Number of Records 6

Loading Order: 1

\begin{tabular}{llllll}
\hline \hline Field Name & PK FK Rqd & Type & Size & Default & Description \\
\hline \hline 1 SystemType_ID & $\square \square \square$ & $\begin{array}{l}\text { AutoNumber } \\
\text { (Long) }\end{array}$ & 4 & $\begin{array}{l}\text { Key that uniquely } \\
\text { identifies a Type of } \\
\text { System }\end{array}$ \\
2 SystemType & $\square \square \square$ & Text & 40 & $\begin{array}{l}\text { Type classification of a } \\
\text { System consisting of one } \\
\text { or more Sites }\end{array}$ \\
3 SystemTypeMemo & $\square \square \square$ & Memo & -- & $\begin{array}{l}\text { Note about a System } \\
\text { Type }\end{array}$
\end{tabular}


Appendix 1: Data Dictionary for the New Jersey Water-Transfer Data System (NJWaTr) —Continued

\section{Table Name: $\quad$ tdxVolumeDetailLabel}

Table Description: User-Extendable List of standardized Labels used for Volume Details

Number of Fields: $\quad 3$

Dependency: Independent

Recursive:

Number of Records 1

Loading Order: 1

\begin{tabular}{llllll}
\hline \hline Field Name & PK FK Rqd & Type & Size & Default & Description \\
\hline \hline $1 \quad$ VolumeDetailLabel_ID & $\square \square \square$ & $\begin{array}{l}\text { AutoNumber } \\
\text { (Long) }\end{array}$ & 4 & $\begin{array}{l}\text { Key that uniquely } \\
\text { identifies a Volume Detail } \\
\text { Label }\end{array}$ \\
2 VolumeDetailLabel & $\square \square \square$ & Text & 50 & $\begin{array}{l}\text { Label describing the } \\
\text { numeric or textual value } \\
\text { of a Volume Detail (for } \\
\text { example, 'Accuracy') } \\
\text { Note about a Volume }\end{array}$ \\
3 & VolumeDetailLabelMemo & $\square \square \square$ & Memo & -- & $\begin{array}{l}\text { Notail Label } \\
\text { Detalu }\end{array}$
\end{tabular}


Appendix 1: Data Dictionary for the New Jersey Water-Transfer Data System (NJWaTr) —Continued

\section{Table Name: $\quad$ tdxVolumeMethod}

Table Description: User-Extendable List of Methods used to measure or estimate a Transfer Volume

Number of Fields: $\quad 4$

Number of Records 42

$\begin{array}{ll}\text { Dependency: } & \text { Partial } \\ \text { Loading Order: } & 2\end{array}$

Recursive: $\square$

\section{Field Name}

2

\begin{tabular}{|c|c|c|c|c|c|c|}
\hline & Field Name & PK FK Rqd & Type & Size & Default & Description \\
\hline 1 & VolumeMethod_ID & $\nabla \square \square$ & $\begin{array}{l}\text { AutoNumber } \\
\text { (Long) }\end{array}$ & 4 & & $\begin{array}{l}\text { Key that uniquely } \\
\text { identifies a Method used } \\
\text { to determine the Volume } \\
\text { of a Transfer }\end{array}$ \\
\hline 2 & VolumeMethodCategory_ID & $\square \square \square$ & Long Integer & 4 & & $\begin{array}{l}\text { Key that uniquely } \\
\text { identifies a Category of } \\
\text { Volume Determination } \\
\text { Methods }\end{array}$ \\
\hline 3 & VolumeMethod & $\square \square \square$ & Text & 200 & & $\begin{array}{l}\text { Name or short description } \\
\text { of the Method used for } \\
\text { determining the Volume } \\
\text { (quantity) of a water } \\
\text { Transfer }\end{array}$ \\
\hline 4 & VolumeMethodMemo & $\square \square \square$ & Memo & -- & & $\begin{array}{l}\text { Note about a Volume } \\
\text { Method }\end{array}$ \\
\hline
\end{tabular}


Appendix 1: Data Dictionary for the New Jersey Water-Transfer Data System (NJWaTr) — Continued

\section{Table Name: tdxVolumeMethodCategory}

Table Description: User-Extendable List of Major Categories of Volume Determination Methods

Number of Fields: $\quad 2$

Number of Records 12
Dependency: Independent

Loading Order: 1
Recursive:

\begin{tabular}{llllll}
\hline \hline Field Name & PK FK Rqd & Type & Size & Default & Description \\
\hline \hline 1 & VolumeMethodCategory_ID & $\square \square \square$ & $\begin{array}{l}\text { AutoNumber } \\
\text { (Long) }\end{array}$ & 4 & $\begin{array}{l}\text { Key that uniquely } \\
\text { identifies a Category of } \\
\text { Volume Determination } \\
\text { Methods }\end{array}$ \\
& & & & $\begin{array}{l}\text { Term that defines a } \\
\text { Category of Volume } \\
\text { Determination Methods }\end{array}$
\end{tabular}


Appendix 1: Data Dictionary for the New Jersey Water-Transfer Data System (NJWaTr) — Continued

\section{Table Name: $\quad$ tdxVolumeUnit}

Table Description: User-Extendable List of Units and common-unit conversion factors used for Transfer Volumes

Number of Fields: 7

Dependency: Partial

Loading Order: 2

Number of Records 10

Loading Order: 2

\begin{tabular}{|c|c|c|c|c|c|c|}
\hline & Field Name & PK FK Rqd & Type & Size & Default & Description \\
\hline 1 & VolumeUnit_ID & $\square \square \square$ & $\begin{array}{l}\text { AutoNumber } \\
\text { (Long) }\end{array}$ & 4 & & $\begin{array}{l}\text { Key that uniquely } \\
\text { identifies a Volume Unit } \\
\text { (units by which raw } \\
\text { Volume values are } \\
\text { originally recorded or } \\
\text { estimated) for a Transfer }\end{array}$ \\
\hline 2 & VolumeUnitDecimal_ID & $\square \square \square$ & Integer & 2 & & $\begin{array}{l}\text { Key that uniquely } \\
\text { identifies a Volume Unit } \\
\text { Decimal component }\end{array}$ \\
\hline 3 & VolumeUnitQuantity_ID & $\square \square \square$ & Integer & 2 & & $\begin{array}{l}\text { Key that uniquely } \\
\text { identifies a Volume Unit } \\
\text { Quantity component }\end{array}$ \\
\hline 4 & VolumeUnitAbbrv & $\square \square \square$ & Text & 50 & & $\begin{array}{l}\text { Abbreviation commonly } \\
\text { used to express a Volume } \\
\text { Unit }\end{array}$ \\
\hline 5 & VolumeUnitPhrase & $\square \square \square$ & Text & 50 & -1 & $\begin{array}{l}\text { Volume Unit Name made } \\
\text { of Decimal, Volume and } \\
\text { Time component terms }\end{array}$ \\
\hline 6 & MGConversion & $\square \square \square$ & Double & 8 & -1 & $\begin{array}{l}\text { Conversion value to be } \\
\text { multiplied against a Raw } \\
\text { Volume Value recorded in } \\
\text { a particular Unit to create } \\
\text { an equivalent value for } \\
\text { that Volume in million } \\
\text { gallons }\end{array}$ \\
\hline 7 & VolumeUnitMemo & $\square \square \square$ & Memo & -- & & Note about a Volume Unit \\
\hline
\end{tabular}


Appendix 2. NJWaTr Domain Table Listings by Subject Area 
Appendix 2. NJWaTr Domain Table Listings by Subject Area

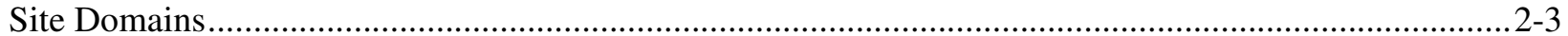

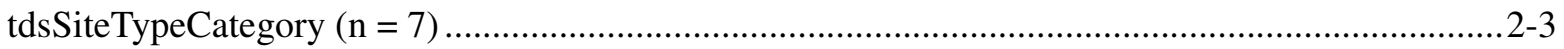

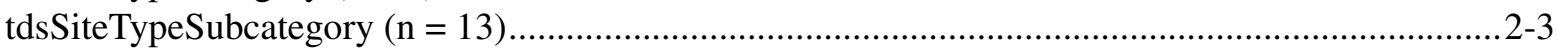

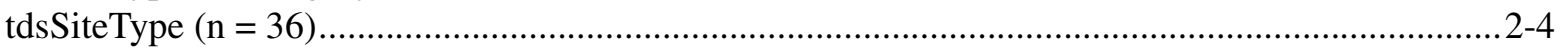

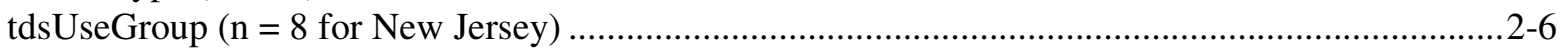

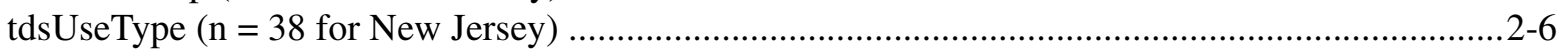

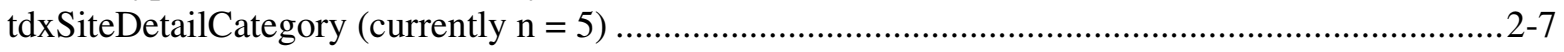

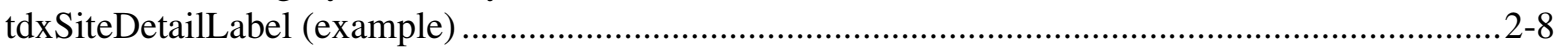

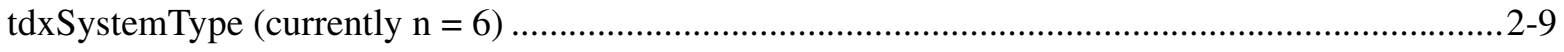

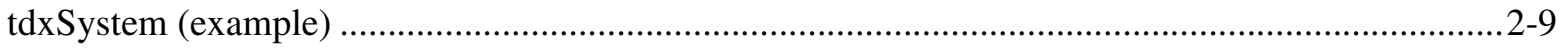

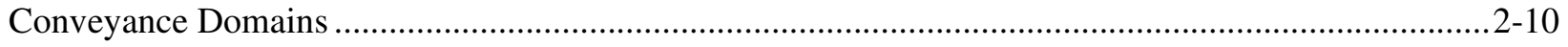

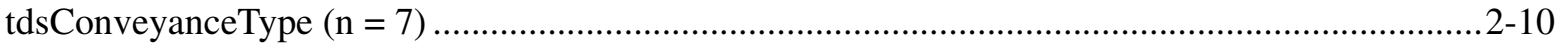

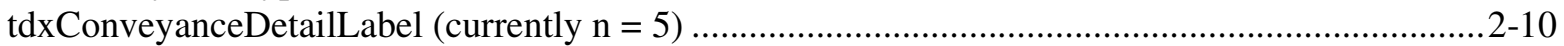

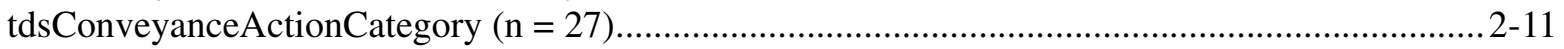

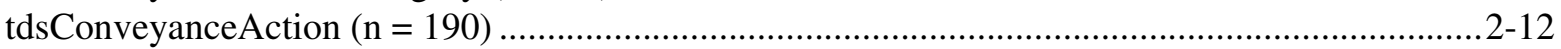

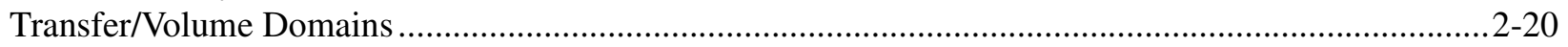

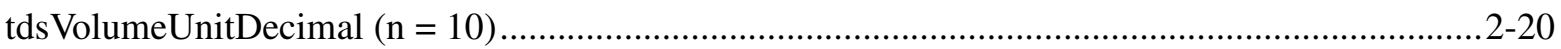

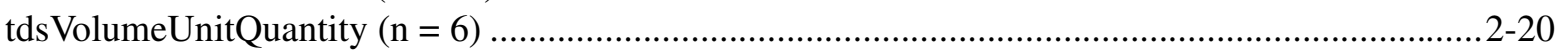

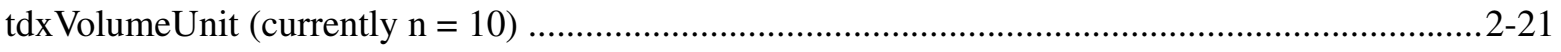

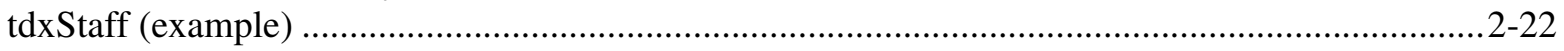

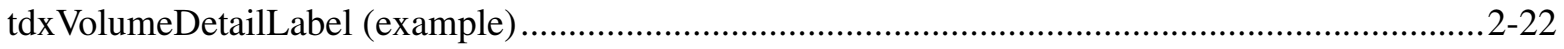

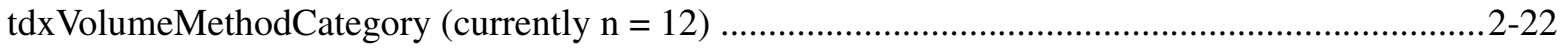

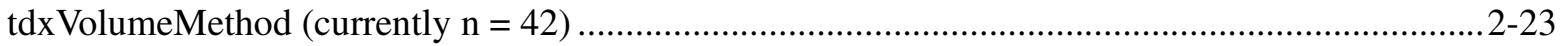

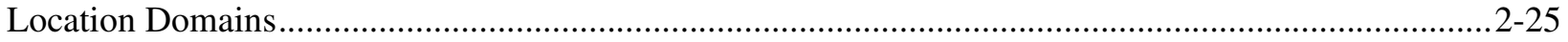

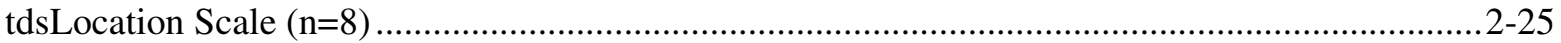

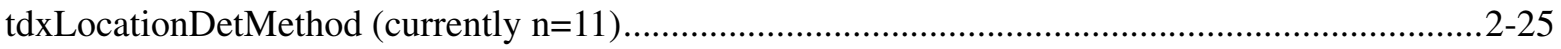

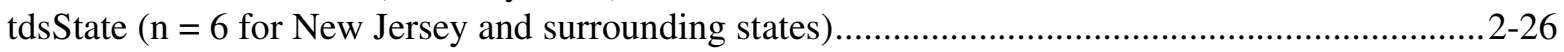

tdsCounty (sample from $\mathrm{n}=178$ for New Jersey and surrounding states) ...................................2-26

tdsMCD (sample from $\mathrm{n}=4,479$ for New Jersey and surrounding states) ..................................2-27

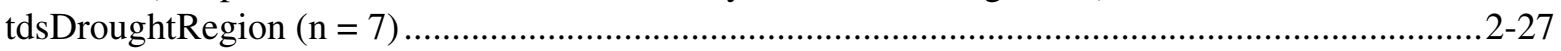

tdsHUC (sample from $\mathrm{n}=935$ for New Jersey and surrounding states) ....................................2-28

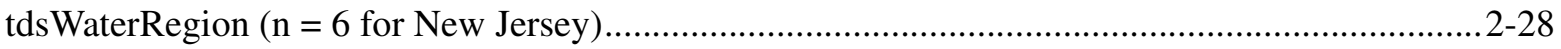

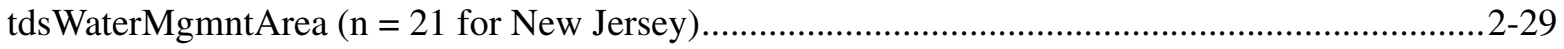

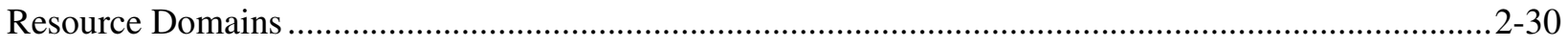

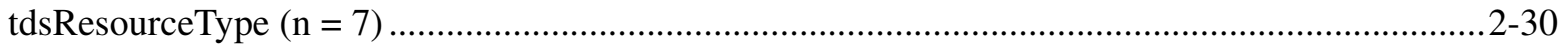

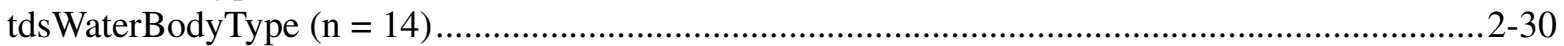

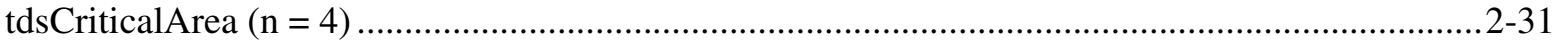

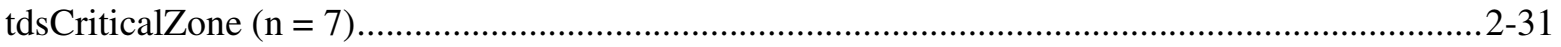

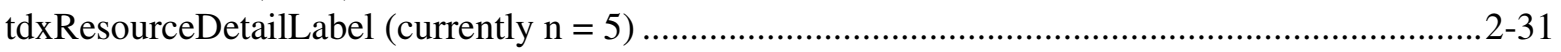

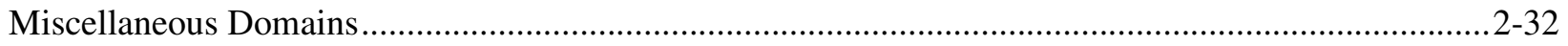

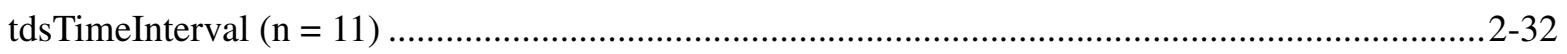

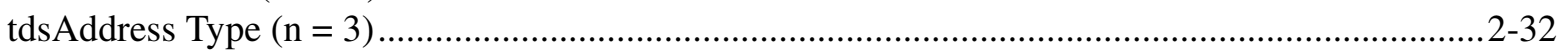

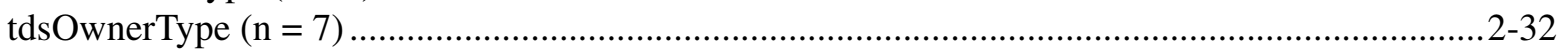

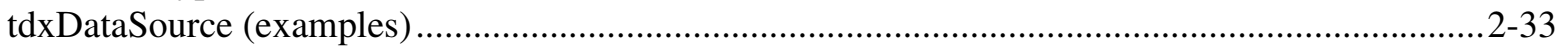

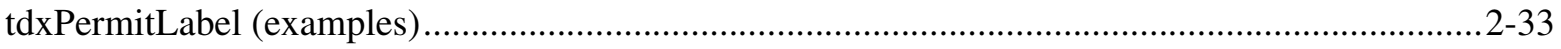

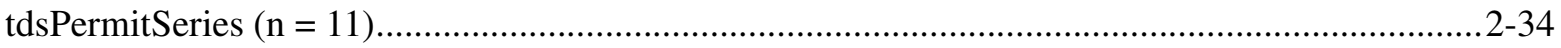

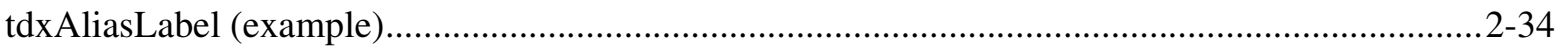


Appendix 2. NJWaTr Domain Table Listings by Subject Area-Continued

\section{Site Domains}

tdsSiteTypeCategory $(\mathrm{n}=7)$

\begin{tabular}{cl}
\hline SiteTypeCategory_ID & \multicolumn{1}{c}{ SiteTypeCategory } \\
\hline 0 & unknown \\
1 & atmosphere \\
2 & resource interactor \\
3 & transfer \\
4 & treatment \\
5 & unaccounted-for water \\
6 & user \\
\hline
\end{tabular}

tdsSiteTypeSubcategory $(\mathrm{n}=13)$

\begin{tabular}{ccl} 
ஸे SiteTypeSubcategory_ID & SiteTypeCategory_ID & SiteTypeSubcategory \\
\hline 0 & 0 & unknown \\
1 & 1 & atmosphere \\
2 & 2 & ground water \\
3 & 2 & spring \\
4 & 2 & surface water \\
5 & 3 & collection \\
6 & 3 & distribution \\
7 & 4 & postuse \\
8 & 4 & preuse \\
9 & 5 & unaccounted-for water \\
10 & 6 & aggregate user \\
11 & 6 & single user \\
12 & 2 & ground and surface water \\
\hline
\end{tabular}


Appendix 2. NJWaTr Domain Table Listings by Subject Area-Continued

Site Domains-Continued

tdsSiteType $(\mathrm{n}=36)$

\begin{tabular}{|c|c|c|c|}
\hline SiteType_ID & SiteTypeSubcategory_ID & SiteType & SiteTypeMemo \\
\hline 0 & 0 & unknown & Unknown - typically used as a place-holder while the true nature of a Site is being investigated \\
\hline 1 & 2 & ground-water withdrawal & Ground-water withdrawal from a general area, as for livestock withdrawals in a county \\
\hline 2 & 2 & wellfield & $\begin{array}{l}\text { A series of wells that are joined together by a manifold metering system and are all completed in the } \\
\text { same aquifer }\end{array}$ \\
\hline 3 & 2 & withdrawal well & A hole in the ground that has a diameter smaller than its depth from which water is withdrawn for use \\
\hline 4 & 2 & recharge well & $\begin{array}{l}\text { A hole in the ground that has a diameter smaller than its depth through which water is pumped back } \\
\text { into the ground }\end{array}$ \\
\hline 5 & 2 & ground-water return flow & Ground-water return flow to a general area, as for domestic return flow in a county \\
\hline 6 & 3 & spring & An opening in the earth from which water flows without pumping \\
\hline 7 & 4 & surface-water withdrawal & Surface-water withdrawal from a general area, as for livestock withdrawals in a county \\
\hline 8 & 4 & intake pipe & A pipe into a surface-water body through which water is removed from the surface-water body \\
\hline 9 & 4 & discharge pipe & A pipe into a surface-water body through which water is returned to the surface-water body \\
\hline 10 & 4 & surface-water return flow & Surface-water return flow to a general area, as for irrigation return flow in a county \\
\hline 11 & 6 & regional distribution system & $\begin{array}{l}\text { A pipe or system of pipes conveying water to another regional distribution system or to more than } \\
\text { one local distribution system }\end{array}$ \\
\hline 12 & 6 & local distribution system & A pipe or system of pipes conveying water to a single MCD or within a single MCD \\
\hline 13 & 5 & regional collection system & $\begin{array}{l}\text { A pipe or system of pipes conveying wastewater from more than one MCD or from another regional } \\
\text { collection system }\end{array}$ \\
\hline 14 & 5 & local collection system & A pipe or system of pipes conveying wastewater from a single MCD \\
\hline 15 & 6 & reclaimed wastewater system & A pipe or system of pipes conveying water from a wastewater-treatment plant to a user \\
\hline 16 & 6 & recycled water system & A pipe or system of pipes conveying water from one user to another user, including itself \\
\hline 17 & 8 & potable-water treatment plant & A treatment plant that prepares water to meet drinking-water standards \\
\hline
\end{tabular}


Appendix 2. NJWaTr Domain Table Listings by Subject Area-Continued

\section{Site Domains-Continued}

tdsSiteType $(\mathrm{n}=36)$-Continued

\begin{tabular}{|c|c|c|c|}
\hline SiteType_ID & SiteTypeSubcategory_ID & SiteType & SiteTypeMemo \\
\hline 18 & 8 & industrial treatment plant & $\begin{array}{l}\text { A treatment plant that prepares water to meet standards that are not necessarily drinking-water } \\
\text { standards--may be higher or lower standards }\end{array}$ \\
\hline 19 & 7 & wastewater-treatment plant & A treatment plant that prepares wastewater for discharge into the hydrologic environment \\
\hline 20 & 11 & single user & $\begin{array}{l}\text { A user for which individual water supply, use, consumptive use, or return flow is measured or } \\
\text { estimated }\end{array}$ \\
\hline 21 & 10 & Aggregate County user & $\begin{array}{l}\text { A group of users, defined by a County boundary, for which supply, use, consumptive use, and return } \\
\text { flow are collectively estimated }\end{array}$ \\
\hline 22 & 1 & Atmosphere (consumptive use) & Atmosphere (consumptive use) represents water that is evaporated or incorporated into products \\
\hline 23 & 9 & Unaccounted-for water & $\begin{array}{l}\text { Unaccounted-for water represents the combination of leakage from distribution systems and public } \\
\text { use of water, such as hydrant flushing, fire fighting, and street sweeping }\end{array}$ \\
\hline 24 & 10 & aggregate MCD user & $\begin{array}{l}\text { A group of users, defined by a Minor Civil Division boundary, for which supply, use, consumptive } \\
\text { use, and return flow are collectively estimated }\end{array}$ \\
\hline 25 & 10 & aggregate HUC user & $\begin{array}{l}\text { A group of users, defined by a Hydrologic Unit boundary, for which supply, use, consumptive use, } \\
\text { and return flow are collectively estimated }\end{array}$ \\
\hline 26 & 10 & aggregate State user & $\begin{array}{l}\text { A group of users, defined by a State boundary, for which supply, use, consumptive use, and return } \\
\text { flow are collectively estimated }\end{array}$ \\
\hline 27 & 4 & Ranney collector & A large diameter well located near a river \\
\hline 28 & 2 & Land application & Disposal of wastewater over a field, as in irrigation \\
\hline 29 & 2 & Recharge basin & Return of freshwater or wastewater into a specially designed basin \\
\hline 30 & 12 & Inflow and Infiltration water & $\begin{array}{l}\text { Inflow and infiltration water represents the combination of inflow from surface water and infiltration } \\
\text { from ground water into a wastewater-collection system }\end{array}$ \\
\hline 31 & 6 & Drinking Water Service Area & Area that is served by one set of distribution pipes and regulated under one BSDW PWS ID number \\
\hline 32 & 11 & BWA Use area & A place-of-use defined by a BWA withdrawal \\
\hline 33 & 6 & DW use area & $\begin{array}{l}\text { A group of users, defined by a DW Service Area boundary, for which supply, use, consumptive use, } \\
\text { and return flow are collectively determined }\end{array}$ \\
\hline 34 & 2 & Intake pipe (ground water) & A pipe into a ground-water-fed pond through which water is removed from the water body \\
\hline 35 & 2 & Discharge pipe (ground water) & A pipe into a ground-water-fed pond through which water is returned to the water body \\
\hline
\end{tabular}


Appendix 2. NJWaTr Domain Table Listings by Subject Area-Continued

Site Domains-Continued

tdsUseGroup ( $\mathrm{n}=8$ for New Jersey)

\begin{tabular}{ccl}
\hline UseGroup_ID & UseGroupCode & \multicolumn{1}{c}{ UseGroup } \\
\hline 0 & -- & undefined \\
1 & 1 & power generation \\
2 & 2 & mining \\
3 & 3 & industrial \\
4 & 4 & commercial \\
5 & 5 & potable supply \\
6 & 6 & irrigation \\
7 & 7 & agricultural \\
\hline
\end{tabular}

$\stackrel{N}{a} \operatorname{tds} U s e T y p e(n=38$ for New Jersey)

\begin{tabular}{cccl}
\hline UseType_ID & UseGroup_ID & UseTypeCode & \multicolumn{1}{c}{ UseType } \\
\hline 0 & 0 & -- & not classified \\
1 & 1 & E & power generation \\
2 & 1 & L & geothermal/heat pump \\
3 & 2 & K & mining \\
4 & 3 & A & air conditioning \\
5 & 3 & D & dewatering \\
6 & 3 & J & cooling (industrial) \\
7 & 3 & N & industrial \\
8 & 3 & W & injection \\
9 & 3 & X & pollution control \\
10 & 4 & C & commercial (non-com) \\
11 & 4 & F & fire \\
12 & 4 & R & recreation (non-comm) \\
13 & 5 & B & bottling \\
14 & 5 & H & domestic \\
15 & 5 & M & medicinal value \\
16 & 5 & O & public non-community \\
17 & 5 & P & public supply \\
& & &
\end{tabular}


Appendix 2. NJWaTr Domain Table Listings by Subject Area-Continued

\section{Site Domains-Continued}

tdsUseType ( $\mathrm{n}=38$ for New Jersey) —Continued

\begin{tabular}{cccl}
\hline UseType_ID & UseGroup_ID & UseTypeCode & \multicolumn{1}{c}{ UseType } \\
\hline 18 & 5 & T & institutional \\
19 & 5 & U & unused \\
20 & 5 & Y & desalination \\
21 & 5 & Z & other \\
22 & 6 & G & golf \\
23 & 6 & I & non-ag irrigation \\
24 & 7 & Q & aquaculture \\
25 & 7 & S & general agriculture \\
26 & 1 & EH & hydro power generation \\
27 & 1 & ET & thermal power generation \\
28 & 7 & SB & blueberries \\
29 & 7 & SC & cranberries \\
30 & 7 & SF & field crops \\
31 & 7 & SG & greenhouse \\
32 & 7 & SI & agriculture irrigation \\
33 & 7 & SS & sod \\
34 & 7 & ST & tree fruit \\
35 & 7 & SV & vegetables, leaf crops \\
36 & 7 & SX & christmas trees \\
37 & 5 & NF & industrial - food processing \\
\hline
\end{tabular}

tdxSiteDetailCategory (currently $\mathrm{n}=5$ )

\begin{tabular}{cl}
\hline SiteDetailCategory_ID & SiteDetailCategory \\
\hline 1 & Count \\
2 & Status \\
3 & Description \\
4 & Coefficient count \\
5 & Percent \\
\hline
\end{tabular}


Appendix 2. NJWaTr Domain Table Listings by Subject Area-Continued

Site Domains-Continued

tdxSiteDetailLabel (example)

[SiteDetailLabelMemo field not shown]

\begin{tabular}{cclcl}
\hline SiteDetailLabel_ID & SiteDetailCategory_ID & \multicolumn{1}{c}{ SiteDetailLabel } & IsNumericDetail & SiteDetailUnit \\
\hline 1 & 1 & Population served & Yes & People \\
2 & 1 & Number of employees & Yes & People \\
3 & 1 & Livestock & Yes & Animals \\
4 & 1 & Acres irrigated-sprinkler & Yes & Acres \\
5 & 2 & Activity status & no & x \\
6 & 3 & Design capacity & Yes & Mgal/d \\
7 & 3 & Storage capacitys & Yes & Mgal \\
8 & 3 & Service connections & Yes & Count \\
9 & 3 & Well depth & Yes & Feet \\
10 & 3 & Diameter & Yes & Inches \\
$\boldsymbol{N}_{\infty}$ & 3 & Pumping rate & Yes & Gal/minute \\
& 3 & Pipe length & Yes & Feet \\
12 & 4 & Aggregate (sand, gravel) & Yes & Tons \\
13 & 1 & Energy & Yes & Kwh \\
14 & 1 & Head & Yes & Feet \\
\hline
\end{tabular}


Appendix 2. NJWaTr Domain Table Listings by Subject Area-Continued

Site Domains-Continued

tdxSystemType (currently $n=6$ )

\begin{tabular}{cll}
\hline SystemType_ID & \multicolumn{1}{c}{ SystemType } & SystemTypeMemo \\
\hline 1 & Public Supplier & \\
2 & Public Wastewater system & \\
3 & Energy Producer & \\
4 & User \\
5 & Town \\
6 & County \\
\hline
\end{tabular}

tdxSystem (example)

\begin{tabular}{|c|c|c|c|c|}
\hline System_ID & SystemType_ID & SystemName & ParentSystem_ID & SystemMemo \\
\hline 1 & 4 & MyIndustry, Inc. & & \\
\hline 2 & 1 & Suburban Water Co. & & \\
\hline
\end{tabular}


Appendix 2. NJWaTr Domain Table Listings by Subject Area-Continued

\section{Conveyance Domains}

tdsConveyanceType $(\mathrm{n}=7)$

\begin{tabular}{ccl}
\hline ConveyanceType_ID & ConveyanceType & \multicolumn{1}{c}{ ConveyanceTypeMemo } \\
\hline 1 & Pipe & Long tube or hollow body for conducting water; closed to atmosphere and earth \\
3 & Aqueduct & $\begin{array}{l}\text { Artificial waterway for draining or irrigating land or for connecting 2 rivers, includes ditches; open to } \\
\text { atmosphere and earth } \\
\text { Large conduit for carrying water; closed to atmosphere, closed to earth }\end{array}$ \\
4 & Conduit & Artificial waterway for conveying water that is open to atmosphere and closed to earth \\
5 & Mixed & $\begin{array}{l}\text { Connection between 2 sites that combines 2 or more conveyance types--varies from being open or closed } \\
\text { throughout its extent } \\
\text { Connection between 2 Sites }\end{array}$ \\
7 & Truck & Two sites connected by truck (water delivered by truck)
\end{tabular}

tdxConveyanceDetailLabel (currently $\mathrm{n}=5$ )

\begin{tabular}{cll}
\hline ConveyanceDetailLabel_ID & ConveyanceDetailLabel & ConveyanceDetailLabelMemo \\
\hline 1 & Pipe size - inches \\
2 & Panal system length - miles & \\
3 & Aqueduct system length - miles \\
4 & Conduit system length - miles \\
5 & Mixed system length - miles \\
\hline
\end{tabular}


Appendix 2. NJWaTr Domain Table Listings by Subject Area-Continued

Conveyance Domains-Continued

tdsConveyanceActionCategory $(\mathrm{n}=27)$

\begin{tabular}{cl}
\hline ConveyanceActionCategory_ID & ConveyanceActionCategory \\
\hline 0 & Unknown \\
1 & collection collection \\
3 & collection treatment \\
4 & consumptive use \\
5 & conveyance loss \\
6 & distribution distribution \\
7 & distribution user \\
8 & distribution treatment \\
9 & infiltration \\
10 & inflow \\
11 & leakage \\
12 & reclaimed wastewater \\
13 & recycled water \\
14 & resource transfer \\
15 & treatment collection \\
16 & treatment distribution \\
17 & treatment resource \\
18 & treatment user \\
19 & user collection \\
20 & user return \\
21 & user treatment \\
22 & withdrawal distribution \\
23 & withdrawal treatment \\
24 & withdrawal user \\
25 & unaccounted for use \\
26 & inflow and infiltration \\
& collection return \\
\hline & \\
\hline &
\end{tabular}


Appendix 2. NJWaTr Domain Table Listings by Subject Area-Continued

\section{Conveyance Domains-Continued}

tdsConveyanceAction $(\mathrm{n}=190)$

\begin{tabular}{|c|c|c|c|c|}
\hline ConveyanceAction_ID & ConveyanceActionCategory_ID & ConveyanceActionPhrase & SiteTypeFromID & SiteTypeToID \\
\hline 0 & 0 & From unknown To unknown & 0 & 0 \\
\hline 1 & 13 & From ground-water withdrawal To recharge well & 1 & 4 \\
\hline 2 & 13 & From ground-water withdrawal To discharge pipe & 1 & 9 \\
\hline 3 & 13 & From wellfield To recharge well & 2 & 4 \\
\hline 4 & 13 & From wellfield To discharge pipe & 2 & 9 \\
\hline 5 & 13 & From withdrawal well To recharge well & 3 & 4 \\
\hline 6 & 13 & From withdrawal well To discharge pipe & 3 & 9 \\
\hline 7 & 13 & From spring To recharge well & 6 & 4 \\
\hline 8 & 13 & From spring To discharge pipe & 6 & 9 \\
\hline 9 & 13 & From surface-water withdrawal To recharge well & 7 & 4 \\
\hline 10 & 13 & From surface-water withdrawal To discharge pipe & 7 & 9 \\
\hline 11 & 13 & From intake pipe To recharge well & 8 & 4 \\
\hline 12 & 13 & From intake pipe To discharge pipe & 8 & 9 \\
\hline 13 & 22 & From ground-water withdrawal To potable-water treatment plant & 1 & 17 \\
\hline 14 & 22 & From wellfield To potable-water treatment plant & 2 & 17 \\
\hline 15 & 22 & From withdrawal well To potable-water treatment plant & 3 & 17 \\
\hline 16 & 22 & From spring To potable-water treatment plant & 6 & 17 \\
\hline 17 & 22 & From surface-water withdrawal To potable-water treatment plant & 7 & 17 \\
\hline 18 & 22 & From intake pipe To potable-water treatment plant & 8 & 17 \\
\hline 19 & 22 & From ground-water withdrawal To industrial treatment plant & 1 & 18 \\
\hline 20 & 22 & From wellfield To industrial treatment plant & 2 & 18 \\
\hline 21 & 22 & From withdrawal well To industrial treatment plant & 3 & 18 \\
\hline 22 & 22 & From spring To industrial treatment plant & 6 & 18 \\
\hline 23 & 22 & From surface-water withdrawal To industrial treatment plant & 7 & 18 \\
\hline 24 & 22 & From intake pipe To industrial treatment plant & 8 & 18 \\
\hline 25 & 21 & From ground-water withdrawal To regional distribution system & 1 & 11 \\
\hline
\end{tabular}


Appendix 2. NJWaTr Domain Table Listings by Subject Area-Continued

Conveyance Domains-Continued

tdsConveyanceAction ( $\mathrm{n}=190)$-Continued

\begin{tabular}{|c|c|c|c|c|}
\hline ConveyanceAction_ID & ConveyanceActionCategory_ID & ConveyanceActionPhrase & SiteTypeFromID & SiteTypeToID \\
\hline 26 & 21 & From wellfield To regional distribution system & 2 & 11 \\
\hline 27 & 21 & From withdrawal well To regional distribution system & 3 & 11 \\
\hline 28 & 21 & From spring To regional distribution system & 6 & 11 \\
\hline 29 & 21 & From surface-water withdrawal To regional distribution system & 7 & 11 \\
\hline 30 & 21 & From intake pipe To regional distribution system & 8 & 11 \\
\hline 31 & 21 & From ground-water withdrawal To local distribution system & 1 & 12 \\
\hline 32 & 21 & From wellfield To local distribution system & 2 & 12 \\
\hline 33 & 21 & From withdrawal well To local distribution system & 3 & 12 \\
\hline 34 & 21 & From spring To local distribution system & 6 & 12 \\
\hline 35 & 21 & From surface-water withdrawal To local distribution system & 7 & 12 \\
\hline 36 & 21 & From intake pipe To local distribution system & 8 & 12 \\
\hline 37 & 23 & From ground-water withdrawal To single user & 1 & 20 \\
\hline 38 & 23 & From wellfield To single user & 2 & 20 \\
\hline 39 & 23 & From withdrawal well To single user & 3 & 20 \\
\hline 40 & 23 & From spring To single user & 6 & 20 \\
\hline 41 & 23 & From surface-water withdrawal To single user & 7 & 20 \\
\hline 42 & 23 & From intake pipe To single user & 8 & 20 \\
\hline 43 & 23 & From ground-water withdrawal To aggregate County user & 1 & 21 \\
\hline 44 & 23 & From wellfield To aggregate County user & 2 & 21 \\
\hline 45 & 23 & From withdrawal well To aggregate County user & 3 & 21 \\
\hline 46 & 23 & From spring To aggregate County user & 6 & 21 \\
\hline 47 & 23 & From surface-water withdrawal To aggregate County user & 7 & 21 \\
\hline 48 & 23 & From intake pipe To aggregate County user & 8 & 21 \\
\hline 49 & 15 & From potable-water treatment plant To regional distribution system & 17 & 11 \\
\hline 50 & 15 & From potable-water treatment plant To local distribution system & 17 & 12 \\
\hline
\end{tabular}


Appendix 2. NJWaTr Domain Table Listings by Subject Area-Continued

Conveyance Domains-Continued

tdsConveyanceAction $(\mathrm{n}=190)$-Continued

\begin{tabular}{|c|c|c|c|c|}
\hline ConveyanceAction_ID & ConveyanceActionCategory_ID & ConveyanceActionPhrase & SiteTypeFromID & SiteTypeToID \\
\hline 51 & 14 & From potable-water treatment plant To local collection system & 17 & 14 \\
\hline 52 & 5 & From regional distribution system To regional distribution system & 11 & 11 \\
\hline 53 & 5 & From regional distribution system To local distribution system & 11 & 12 \\
\hline 54 & 6 & From local distribution system To single user & 12 & 20 \\
\hline 55 & 7 & From local distribution system To industrial treatment plant & 12 & 18 \\
\hline 56 & 17 & From industrial treatment plant To single user & 18 & 20 \\
\hline 57 & 6 & From local distribution system To aggregate County user & 12 & 21 \\
\hline 58 & 18 & From single user To local collection system & 20 & 14 \\
\hline 59 & 18 & From aggregate County user To local collection system & 21 & 14 \\
\hline 60 & 19 & From single user To recharge well & 20 & 4 \\
\hline 61 & 19 & From single user To ground-water return flow & 20 & 5 \\
\hline 62 & 19 & From single user To discharge pipe & 20 & 9 \\
\hline 63 & 19 & From single user To surface-water return flow & 20 & 10 \\
\hline 64 & 19 & From aggregate County user To recharge well & 21 & 4 \\
\hline 65 & 19 & From aggregate County user To ground-water return flow & 21 & 5 \\
\hline 66 & 19 & From aggregate County user To discharge pipe & 21 & 9 \\
\hline 67 & 19 & From aggregate County user To surface-water return flow & 21 & 10 \\
\hline 68 & 1 & From local collection system To regional collection system & 14 & 13 \\
\hline 69 & 1 & From regional collection system To regional collection system & 13 & 13 \\
\hline 70 & 2 & From local collection system To wastewater-treatment plant & 14 & 19 \\
\hline 71 & 2 & From regional collection system To wastewater-treatment plant & 13 & 19 \\
\hline 72 & 20 & From single user To wastewater-treatment plant & 20 & 19 \\
\hline 73 & 14 & From industrial treatment plant To local collection system & 18 & 14 \\
\hline 74 & 11 & From wastewater-treatment plant To reclaimed wastewater system & 19 & 15 \\
\hline
\end{tabular}


Appendix 2. NJWaTr Domain Table Listings by Subject Area-Continued

Conveyance Domains-Continued

tdsConveyanceAction ( $\mathrm{n}=190)$-Continued

\begin{tabular}{|c|c|c|c|c|}
\hline ConveyanceAction_ID & ConveyanceActionCategory_ID & ConveyanceActionPhrase & SiteTypeFromID & SiteTypeToID \\
\hline 75 & 11 & From reclaimed wastewater system To single user & 15 & 20 \\
\hline 76 & 11 & From reclaimed wastewater system To aggregate County user & 15 & 21 \\
\hline 77 & 12 & From single user To recycled water system & 20 & 16 \\
\hline 78 & 12 & From recycled water system To single user & 16 & 20 \\
\hline 79 & 16 & From industrial treatment plant To recharge well & 18 & 4 \\
\hline 80 & 16 & From industrial treatment plant To ground-water return flow & 18 & 5 \\
\hline 81 & 16 & From industrial treatment plant To discharge pipe & 18 & 9 \\
\hline 82 & 16 & From industrial treatment plant To surface-water return flow & 18 & 10 \\
\hline 83 & 16 & From wastewater-treatment plant To recharge well & 19 & 4 \\
\hline 84 & 16 & From wastewater-treatment plant To ground-water return flow & 19 & 5 \\
\hline 85 & 16 & From wastewater-treatment plant To discharge pipe & 19 & 9 \\
\hline 86 & 16 & From wastewater-treatment plant To surface-water return flow & 19 & 10 \\
\hline 87 & 8 & From ground-water withdrawal To local collection system & 1 & 14 \\
\hline 88 & 8 & From ground-water withdrawal To regional collection system & 1 & 13 \\
\hline 89 & 9 & From surface-water withdrawal To local collection system & 7 & 14 \\
\hline 90 & 9 & From surface-water withdrawal To regional collection system & 7 & 13 \\
\hline 91 & 10 & From local distribution system To ground-water return flow & 12 & 5 \\
\hline 92 & 10 & From regional distribution system To ground-water return flow & 11 & 5 \\
\hline 93 & 10 & From local collection system To ground-water return flow & 14 & 5 \\
\hline 94 & 10 & From regional collection system To ground-water return flow & 13 & 5 \\
\hline 95 & 3 & From single user To Atmosphere (consumptive use) & 20 & 22 \\
\hline 96 & 3 & From aggregate County user To Atmosphere (consumptive use) & 21 & 22 \\
\hline
\end{tabular}


Appendix 2. NJWaTr Domain Table Listings by Subject Area-Continued

Conveyance Domains-Continued

tdsConveyanceAction ( $\mathrm{n}=190)$-Continued

\begin{tabular}{|c|c|c|c|c|}
\hline ConveyanceAction_ID & ConveyanceActionCategory_ID & ConveyanceActionPhrase & SiteTypeFromID & SiteTypeToID \\
\hline 97 & 4 & From ground-water withdrawal To ground-water return flow & 1 & 5 \\
\hline 98 & 4 & From surface-water withdrawal To ground-water return flow & 7 & 5 \\
\hline 99 & 19 & From aggregate MCD user To recharge well & 24 & 4 \\
\hline 100 & 19 & From aggregate MCD user To ground-water return flow & 24 & 5 \\
\hline 101 & 19 & From aggregate $\mathrm{MCD}$ user To discharge pipe & 24 & 9 \\
\hline 102 & 19 & From aggregate MCD user To surface-water return flow & 24 & 10 \\
\hline 103 & 18 & From aggregate MCD user To local collection system & 24 & 14 \\
\hline 104 & 3 & From aggregate MCD user To Atmosphere (consumptive use) & 24 & 22 \\
\hline 105 & 19 & From aggregate HUC user To recharge well & 25 & 4 \\
\hline 106 & 19 & From aggregate HUC user To ground-water return flow & 25 & 5 \\
\hline 107 & 19 & From aggregate HUC user To discharge pipe & 25 & 9 \\
\hline 108 & 19 & From aggregate HUC user To surface-water return flow & 25 & 10 \\
\hline 109 & 18 & From aggregate HUC user To local collection system & 25 & 14 \\
\hline 110 & 3 & From aggregate HUC user To Atmosphere (consumptive use) & 25 & 22 \\
\hline 111 & 19 & From aggregate State user To recharge well & 26 & 4 \\
\hline 112 & 19 & From aggregate State user To ground-water return flow & 26 & 5 \\
\hline 113 & 19 & From aggregate State user To discharge pipe & 26 & 9 \\
\hline 114 & 19 & From aggregate State user To surface-water return flow & 26 & 10 \\
\hline 115 & 18 & From aggregate State user To local collection system & 26 & 14 \\
\hline 116 & 3 & From aggregate State user To Atmosphere (consumptive use) & 26 & 22 \\
\hline 117 & 13 & From Ranney collector To recharge well & 27 & 4 \\
\hline 118 & 13 & From Ranney collector To discharge pipe & 27 & 9 \\
\hline 119 & 21 & From Ranney collector To regional distribution system & 27 & 11 \\
\hline 120 & 21 & From Ranney collector To local distribution system & 27 & 12 \\
\hline 121 & 22 & From Ranney collector To potable-water treatment plant & 27 & 17 \\
\hline 122 & 22 & From Ranney collector To industrial treatment plant & 27 & 18 \\
\hline
\end{tabular}


Appendix 2. NJWaTr Domain Table Listings by Subject Area-Continued

Conveyance Domains-Continued

\begin{tabular}{|c|c|c|c|c|}
\hline ConveyanceAction_ID & ConveyanceActionCategory_ID & ConveyanceActionPhrase & SiteTypeFromID & SiteTypeToID \\
\hline 123 & 23 & From Ranney collector To single user & 27 & 20 \\
\hline 124 & 23 & From Ranney collector To aggregate County user & 27 & 21 \\
\hline 125 & 23 & From ground-water withdrawal To aggregate MCD user & 1 & 24 \\
\hline 126 & 23 & From wellfield To aggregate MCD user & 2 & 24 \\
\hline 127 & 23 & From withdrawal well To aggregate MCD user & 3 & 24 \\
\hline 128 & 23 & From spring To aggregate MCD user & 6 & 24 \\
\hline 129 & 23 & From surface-water withdrawal To aggregate MCD user & 7 & 24 \\
\hline 130 & 23 & From intake pipe To aggregate MCD user & 8 & 24 \\
\hline 131 & 6 & From local distribution system To aggregate MCD user & 12 & 24 \\
\hline 132 & 11 & From reclaimed wastewater system To aggregate MCD user & 15 & 24 \\
\hline 133 & 23 & From ground-water withdrawal To aggregate HUC user & 1 & 25 \\
\hline 134 & 23 & From wellfield To aggregate HUC user & 2 & 25 \\
\hline 135 & 23 & From withdrawal well To aggregate HUC user & 3 & 25 \\
\hline 136 & 23 & From spring To aggregate HUC user & 6 & 25 \\
\hline 137 & 23 & From surface-water withdrawal To aggregate HUC user & 7 & 25 \\
\hline 138 & 23 & From intake pipe To aggregate HUC user & 8 & 25 \\
\hline 139 & 6 & From local distribution system To aggregate HUC user & 12 & 25 \\
\hline 140 & 11 & From reclaimed wastewater system To aggregate HUC user & 15 & 25 \\
\hline 141 & 23 & From ground-water withdrawal To aggregate State user & 1 & 26 \\
\hline 142 & 23 & From wellfield To aggregate State user & 2 & 26 \\
\hline 143 & 23 & From withdrawal well To aggregate State user & 3 & 26 \\
\hline 144 & 23 & From spring To aggregate State user & 6 & 26 \\
\hline 145 & 23 & From surface-water withdrawal To aggregate State user & 7 & 26 \\
\hline 146 & 23 & From intake pipe To aggregate State user & 8 & 26 \\
\hline 147 & 6 & From local distribution system To aggregate State user & 12 & 26 \\
\hline 148 & 11 & From reclaimed wastewater system To aggregate State user & 15 & 26 \\
\hline 149 & 24 & From regional distribution system To Unaccounted-for water & 11 & 23 \\
\hline
\end{tabular}


Appendix 2. NJWaTr Domain Table Listings by Subject Area-Continued

Conveyance Domains-Continued

tdsConveyanceAction $(\mathrm{n}=190)$-Continued

\begin{tabular}{|c|c|c|c|c|}
\hline ConveyanceAction_ID & ConveyanceActionCategory_ID & ConveyanceActionPhrase & SiteTypeFromID & SiteTypeToID \\
\hline 150 & 24 & From local distribution system To Unaccounted-for water & 12 & 23 \\
\hline 151 & 25 & From Inflow and Infiltration water To regional collection system & 30 & 13 \\
\hline 152 & 25 & From Inflow and Infiltration water To local collection system & 30 & 14 \\
\hline 153 & 11 & From industrial treatment plant To Land application & 18 & 28 \\
\hline 154 & 11 & From wastewater-treatment plant To Land application & 19 & 28 \\
\hline 155 & 11 & From single user To Land application & 20 & 28 \\
\hline 156 & 11 & From aggregate County user To Land application & 21 & 28 \\
\hline 157 & 11 & From aggregate MCD user To Land application & 24 & 28 \\
\hline 158 & 11 & From aggregate HUC user To Land application & 25 & 28 \\
\hline 159 & 11 & From aggregate State user To Land application & 26 & 28 \\
\hline 160 & 13 & From ground-water withdrawal To Recharge basin & 1 & 29 \\
\hline 161 & 13 & From wellfield To Recharge basin & 2 & 29 \\
\hline 162 & 13 & From withdrawal well To Recharge basin & 3 & 29 \\
\hline 163 & 13 & From spring To Recharge basin & 6 & 29 \\
\hline 164 & 13 & From surface-water withdrawal To Recharge basin & 7 & 29 \\
\hline 165 & 13 & From intake pipe To Recharge basin & 8 & 29 \\
\hline 166 & 13 & From industrial treatment plant To Recharge basin & 18 & 29 \\
\hline 167 & 16 & From wastewater-treatment plant To Recharge basin & 19 & 29 \\
\hline 168 & 16 & From single user To Recharge basin & 20 & 29 \\
\hline 169 & 19 & From aggregate County user To Recharge basin & 21 & 29 \\
\hline 170 & 19 & From aggregate MCD user To Recharge basin & 24 & 29 \\
\hline 171 & 19 & From aggregate HUC user To Recharge basin & 25 & 29 \\
\hline 172 & 19 & From aggregate State user To Recharge basin & 26 & 29 \\
\hline 173 & 23 & From withdrawal well To Drinking Water Service Area & 3 & 31 \\
\hline 174 & 23 & From withdrawal well To BWA use area & 3 & 32 \\
\hline 175 & 23 & From spring To BWA use area & 6 & 32 \\
\hline 176 & 23 & From intake pipe To Drinking Water Service Area & 8 & 31 \\
\hline
\end{tabular}


Appendix 2. NJWaTr Domain Table Listings by Subject Area-Continued

Conveyance Domains-Continued

tdsConveyanceAction ( $\mathrm{n}=190)$-Continued

\begin{tabular}{|c|c|c|c|c|}
\hline ConveyanceAction_ID & ConveyanceActionCategory_ID & ConveyanceActionPhrase & SiteTypeFromID & SiteTypeToID \\
\hline 177 & 23 & From intake pipe To BWA use area & 8 & 32 \\
\hline 178 & 23 & From unknown To Drinking Water Service Area & 0 & 31 \\
\hline 179 & 23 & From unknown To BWA use area & 0 & 32 \\
\hline 180 & 23 & From ground-water withdrawal To unknown & 1 & 0 \\
\hline 181 & 3 & From BWA use area To Atmosphere (consumptive use) & 32 & 22 \\
\hline 182 & 5 & From Drinking Water Service Area To Drinking Water Service Area & 31 & 31 \\
\hline 183 & 6 & From Drinking Water Service Area To DW Use Area & 31 & 33 \\
\hline 184 & 24 & From Drinking Water Service Area To Unaccounted-for water & 31 & 23 \\
\hline 185 & 3 & From DW Use Area To Atmosphere (consumptive use) & 33 & 22 \\
\hline 186 & 26 & From regional collection system To discharge pipe & 13 & 9 \\
\hline 187 & 21 & From spring To Drinking Water Service Area & 6 & 31 \\
\hline 188 & 18 & From DW Use Area To regional distribution system & 33 & 11 \\
\hline 189 & 25 & From Inflow and Infiltration water To regional distribution system & 30 & 11 \\
\hline
\end{tabular}


Appendix 2. NJWaTr Domain Table Listings by Subject Area-Continued

\section{Transfer/Volume Domains}

tds VolumeUnitDecimal $(\mathrm{n}=10)$

\begin{tabular}{cllc}
\hline VolumeUnitDecimal_ID & DecimalUnit & ConversionToMillion \\
\hline 0 & one & 0.000001 \\
1 & ten & 0.00001 \\
2 & hundred & 0.0001 \\
3 & thousand & 0.001 \\
4 & ten thousand & 0.01 \\
5 & hundred thousand & 0.1 \\
6 & million & 1 \\
7 & ten million & 10 \\
& & hundred million & 100 \\
& & billion & 1000 \\
\hline \multirow{0}{*}{} & & &
\end{tabular}

tds VolumeUnitQuantity $(\mathrm{n}=6)$

\begin{tabular}{clc}
\hline VolumeUnitQuantity_ID & QuantityUnit & ConversionToGallon \\
\hline 1 & gallons & 1 \\
2 & cubic feet & 7.48 \\
3 & acre-feet & 325851 \\
4 & acre-inch & 27154 \\
5 & cubic inch & 0.00433 \\
6 & liter & 0.2642 \\
\hline
\end{tabular}


Appendix 2. NJWaTr Domain Table Listings by Subject Area-Continued

Transfer/Volume Domains-Continued

tdx VolumeUnit (currently $\mathrm{n}=10$ )

[VolumeUnitMemo field not shown]

\begin{tabular}{|c|c|c|c|c|c|}
\hline VolumeUnit_ID & VolumeUnitDecimal_ID & VolumeUnitQuantity_ID & VolumeUnitAbbrv & VolumeUnitPhrase & MGConversion \\
\hline 1 & 6 & 1 & Mgal & million gallons & 1 \\
\hline 2 & 0 & 2 & $\mathrm{cft}$ & cubic feet & 0.00000748 \\
\hline 3 & 3 & 2 & Tcf & thousand cubic feet & 0.00748 \\
\hline 4 & 0 & 3 & acre-feet & acre-feet & 0.325851 \\
\hline 5 & 3 & 3 & Tacre-feet & thousand acre-feet & 325.851 \\
\hline 6 & 0 & 1 & gal & gallons & 0.000001 \\
\hline 7 & 3 & 1 & Tgal & thousand gallons & 0.001 \\
\hline 8 & 0 & 4 & acre-inch & acre-inch & 0.027154 \\
\hline 9 & 0 & 5 & cinch & cubic inch & 0.00000000433 \\
\hline 10 & 9 & 1 & Bgal & billion gallons & 1000 \\
\hline
\end{tabular}


Appendix 2. NJWaTr Domain Table Listings by Subject Area-Continued

Transfer/Volume Domains-Continued

tdxStaff (example)

\begin{tabular}{ccccc}
\hline Staff_ID & StaffInitials & StaffName & StaffAffiliation & StaffMemo \\
\hline 1 & NJGS & NJGS & NJGS & Generic entry for NJGS staff \\
\hline
\end{tabular}

tdx VolumeDetailLabel (example)

\begin{tabular}{ccc}
\hline VolumeDetailLabel_ID & VolumeDetailLabel & VolumeDetailLabelMemo \\
\hline 1 & Accuracy \\
\hline
\end{tabular}

ì tdx VolumeMethodCategory (currently $\mathrm{n}=12$ )

\begin{tabular}{cl}
\hline VolumeMethodCategory_ID & VolumeMethodCategory \\
\hline 0 & Unknown Method \\
1 & Metered \\
2 & Field estimate \\
3 & Coefficient estimate \\
4 & Reported \\
5 & Intuition \\
6 & Percent of metered \\
7 & Percent of derived value \\
8 & Difference between metered values \\
9 & Difference between derived values \\
10 & Permit \\
11 & Volumetric \\
\hline
\end{tabular}


Appendix 2. NJWaTr Domain Table Listings by Subject Area-Continued

\section{Transfer/Volume Domains-Continued}

tdx VolumeMethod (currently $n=42$ )

[VolumeMethodMemo field not shown]

\begin{tabular}{|c|c|c|}
\hline VolumeMethod_ID & VolumeMethodCategory_ID & VolumeMethod \\
\hline 0 & 0 & Unknown method \\
\hline 1 & 0 & Unknown source, unknown method \\
\hline 2 & 1 & Uncalibrated cumulative meter \\
\hline 3 & 1 & Calibrated instantaneous meter with time meter \\
\hline 4 & 1 & Calibrated instantaneous meter with time estimate \\
\hline 5 & 1 & Uncalibrated instantaneous meter with time meter \\
\hline 6 & 1 & Uncalibrated instantaneous meter with time estimate \\
\hline 7 & 2 & Estimated pumping rate with time meter \\
\hline 8 & 2 & Estimated pumping rate with time estimate \\
\hline 9 & 3 & IWR-MAIN coefficient with Dun-Bradstreet values \\
\hline 10 & 3 & IWR-MAIN coefficient with Census Bureau values \\
\hline 11 & 3 & Local coefficient with Dun-Bradstreet values \\
\hline 12 & 3 & Local coefficient with Census Bureau values \\
\hline 13 & 3 & Power coefficient with power-consumption data \\
\hline 14 & 3 & Agriculture coefficient with landsat data \\
\hline 15 & 3 & Livestock coefficient with Agriculture Census data \\
\hline 16 & 3 & Agriculture coefficient with Agriculture Census data \\
\hline 17 & 3 & NWUIP coefficient with Census data \\
\hline 18 & 1 & Calibrated cumulative meter \\
\hline 19 & 7 & $\begin{array}{l}\text { IWR-MAIN coefficient applied to Dun-Bradstreet values to determine water use and use Census Bureau } \\
\text { data to proportion public supply/disposal from self supply/disposal }\end{array}$ \\
\hline 20 & 6 & Metered withdrawal data multiplied by consumptive-use percentage \\
\hline 21 & 10 & Permitted volume \\
\hline 22 & 6 & Metered withdrawal data multiplied by local distribution system percentage \\
\hline 23 & 6 & Metered delivery data multiplied by consumptive-use percentage \\
\hline 24 & 7 & Aggregated data multiplied by land- and employee-based percentage \\
\hline 25 & 7 & Water-use value multiplied by consumptive-use percentage \\
\hline 26 & 2 & Stream gage-USGS \\
\hline
\end{tabular}


Appendix 2. NJWaTr Domain Table Listings by Subject Area-Continued

\section{Transfer/Volume Domains-Continued}

tdx VolumeMethod (currently $\mathrm{n}=42$ ) —Continued

\begin{tabular}{|c|c|c|}
\hline VolumeMethod_ID & VolumeMethodCategory_ID & VolumeMethod \\
\hline 27 & 2 & Stream gage-other than USGS \\
\hline 28 & 11 & Known volume, such as truck times the number of trucks over a specified time periode \\
\hline 29 & 7 & Public supply withdrawals multiplied by unaccounted-for use percentage \\
\hline 30 & 8 & Difference between metered withdrawals and billed consumption \\
\hline 31 & 6 & Metered wastewater values multiplied by population-based percentage \\
\hline 32 & 7 & $\begin{array}{l}\text { Percent Total aggregate water use multiplied by percent of wells in different aquifers for MCD from VTDEC, WSD well } \\
\text { inventory database }\end{array}$ \\
\hline 33 & 7 & Difference between Census population and population on public supply. \\
\hline 34 & 9 & Difference between registered withdrawals and public-supply deliveries, factoring in all connected MCDs \\
\hline 35 & 7 & Volume method \#20 times percentage: $95 \%$ bedrock and 5\%glacial deposits aquifer \\
\hline 36 & 9 & Average irrigation needs less actual precipitation; gw/sw split by guestimate \\
\hline 37 & 3 & Livestock coefficient with Agriculture Census data plus count of dairy cows (from VTDOA) times water-use coefficient \\
\hline 38 & 1 & Metered data; unknown type \\
\hline 39 & 9 & Trying to correct an error, placehold volume method \\
\hline 40 & 3 & SDWIS population times $70 \mathrm{gal} /$ person/day, divided among wells as appropriate, $85 \%$ return flow \\
\hline 41 & 2 & Estimated based on reported other months \\
\hline
\end{tabular}


Appendix 2. NJWaTr Domain Table Listings by Subject Area-Continued

\section{Location Domains}

tdsLocation Scale $(\mathrm{n}=8)$

\begin{tabular}{cl}
\hline LocationScale_ID & LocationScale \\
\hline 0 & Unknown Scale \\
1 & Point \\
2 & MCD/Town \\
3 & County \\
4 & State \\
5 & HUC/Watershed \\
6 & Irregular Area \\
7 & Undefined Area \\
\hline
\end{tabular}

芩

tdxLocationDetMethod (currently $n=11$ )

\begin{tabular}{cl}
\hline LocationDetMethod_ID & \multicolumn{1}{c}{ LocationDetMethod } \\
\hline 0 & Unknown Method \\
1 & Centroid of MCD \\
2 & Centroid of County \\
3 & Centroid of HUC/Watershed \\
4 & GPS field \\
5 & Topographic map \\
6 & Surveyed in \\
7 & Atlas software \\
8 & Dun \& Bradstreet Info Service \\
9 & EPA-SDWIS database \\
10 & Geographic Information System \\
\hline
\end{tabular}


Appendix 2. NJWaTr Domain Table Listings by Subject Area-Continued

Location Domains-Continued

tdsState ( $\mathrm{n}=6$ for New Jersey and surrounding states)

[Not shown are the fields StateLatitude and StateLongitude]

\begin{tabular}{clccl}
\hline State_ID & CountryAbbrv & StateCode & StateAbbrv & \multicolumn{1}{c}{ StateName } \\
\hline 0 & USA & 00 & XX & No State Identified \\
1 & USA & 34 & NJ & New Jersey \\
2 & USA & 10 & DE & Delaware \\
3 & USA & 24 & MD & Maryland \\
4 & USA & 36 & NY & New York \\
5 & USA & 42 & PA & Pennsylvania \\
\hline
\end{tabular}

tdsCounty (sample from $\mathrm{n}=178$ for New Jersey and surrounding states)

Nั [Not shown are the fields CountyLatitude and CountyLongitude]

\begin{tabular}{ccccll}
\hline County_ID & State_ID & StateCountyCode & CountyCode & CountyName & CountyShortName \\
\hline 28 & 1 & 34001 & 001 & Atlantic County & Atlantic \\
29 & 1 & 34003 & 003 & Bergen County & Bergen \\
30 & 1 & 34005 & 005 & Burlington County & Burlington \\
31 & 1 & 34007 & 007 & Camden County & Camden \\
32 & 1 & 34009 & 009 & Cape May County & Cape May \\
33 & 1 & 34011 & 011 & Cumberland County & Cumberland \\
\hline
\end{tabular}


Appendix 2. NJWaTr Domain Table Listings by Subject Area-Continued

Location Domains-Continued

tdsMCD (sample from $n=4,479$ for New Jersey and surrounding states)

[Not shown are the fields MCDLatitude and MCDLongitude]

\begin{tabular}{|c|c|c|c|c|c|c|c|}
\hline MCD_ID & County_ID & DroughtRegion_ID & StateMCDCode & MCDCode & MCDType & MCDName & MCDShortName \\
\hline 322 & 28 & 6 & 3400100 & 00100 & City & Absecon city & Absecon \\
\hline 323 & 28 & 6 & 3402080 & 02080 & City & Atlantic City city & Atlantic City \\
\hline 324 & 28 & 6 & 3407810 & 07810 & City & Brigantine city & Brigantine \\
\hline 325 & 28 & 6 & 3408680 & 08680 & Borough & Buena borough & Buena \\
\hline
\end{tabular}

tdsDroughtRegion $(\mathrm{n}=7)$

\begin{tabular}{ccll}
\hline & DroughtRegion_ID & DroughtRegionCode & DroughtRegion \\
\cline { 2 - 4 } & 0 & $\mathrm{n} / \mathrm{a}$ & Not Applicable \\
1 & $\mathrm{NE}$ & Northeast \\
2 & $\mathrm{NW}$ & Northwest \\
3 & $\mathrm{C}$ & Central \\
4 & $\mathrm{SW}$ & Southwest \\
5 & $\mathrm{CN}$ & Coastal, North \\
6 & $\mathrm{CS}$ & Coastal, South \\
\hline
\end{tabular}


Appendix 2. NJWaTr Domain Table Listings by Subject Area-Continued

Location Domains-Continued

tdsHUC (sample from $\mathrm{n}=935$ for New Jersey and surrounding states)

[Not shown are the fields HUC11Name, HUC8, and HUC8Name]

\begin{tabular}{ccclc}
\hline HUC_ID & WaterMgmntArea_ID & WatershedCode & HUC14 & \multicolumn{1}{c}{ HUC14Name } \\
\hline 2 & 0 & $02 \mathrm{BA01}$ & 02020007010010 & Wallkill R/Lake Mohawk (above Sparta Sta) \\
3 & 0 & $02 \mathrm{BA02}$ & 02020007010020 & Wallkill R (Ogdensburg to SpartaStation) \\
4 & 0 & $02 \mathrm{BA03}$ & 02020007010030 & Franklin Pond Creek \\
5 & 0 & $02 \mathrm{BA04}$ & 02020007010040 & Wallkill R (Hamburg SW Bdy to Ogdensburg) \\
6 & 0 & $02 \mathrm{BA05}$ & 02020007010050 & Hardistonville tribs \\
\hline
\end{tabular}

tdsWaterRegion ( $\mathrm{n}=6$ for New Jersey)

\begin{tabular}{|c|c|c|}
\hline WaterRegion_ID & WaterRegionCode & WaterRegion \\
\hline 0 & -- & Unknown \\
\hline 1 & 1 & Passaic \\
\hline 2 & 2 & Raritan \\
\hline 3 & 3 & Atlantic Coastal \\
\hline 4 & 4 & Upper Delaware \\
\hline 5 & 5 & Lower Delaware \\
\hline
\end{tabular}


Appendix 2. NJWaTr Domain Table Listings by Subject Area-Continued

Location Domains-Continued

tdsWaterMgmntArea ( $\mathrm{n}=21$ for New Jersey)

\begin{tabular}{|c|c|c|c|}
\hline WaterMgmntArea_ID & WaterRegion_ID & WaterMgmntAreaCode & WaterMgmntArea \\
\hline 0 & 0 & -- & Unknown \\
\hline 1 & 4 & 1 & Upper Delaware \\
\hline 2 & 4 & 2 & Walkill, Pochuck, and Papakating \\
\hline 3 & 1 & 3 & Pompton, Pequannock, Wanaque, and Ramapo \\
\hline 4 & 1 & 4 & Lower Passaic and Saddle \\
\hline 5 & 1 & 5 & Hackensack and Pascack \\
\hline 6 & 1 & 6 & Upper Passaic, Whippany, and Rockaway \\
\hline 7 & 2 & 7 & Elizabeth, Rahway, and Woodbridge \\
\hline 8 & 2 & 8 & North and Sourth Branch Raritan \\
\hline 9 & 2 & 9 & Lower Raritan, South, and Lawrence \\
\hline 10 & 2 & 10 & Millstone \\
\hline 11 & 4 & 11 & Central Delaware \\
\hline 12 & 3 & 12 & Monmouth County \\
\hline 13 & 3 & 13 & Barnegat Bay \\
\hline 14 & 3 & 14 & Mullica and Wading \\
\hline 15 & 3 & 15 & Great Egg Harbor and Tuckahoe \\
\hline 16 & 3 & 16 & Cape May County \\
\hline 17 & 5 & 17 & Maruice, Salem, and Cohansey \\
\hline 18 & 5 & 18 & Lower Delaware \\
\hline 19 & 5 & 19 & Rancocas \\
\hline 20 & 5 & 20 & Crosswicks \\
\hline
\end{tabular}


Appendix 2. NJWaTr Domain Table Listings by Subject Area-Continued Resource Domains

tdsResourceType $(\mathrm{n}=7)$

\begin{tabular}{cccl}
\hline ResourceType_ID & GWorSW & Salinity & ResourceType \\
\hline 0 & -- & -- & Unknown \\
1 & GW & FR & Ground water, fresh \\
2 & GW & BR & Ground-water, brackish \\
3 & GW & SA & Ground-water, saline \\
4 & SW & FR & Surface-water, fresh \\
5 & SW & BR & Surface-water, brackish \\
6 & SW & SA & Surface-water, saline \\
\hline
\end{tabular}

岕 tdsWaterBodyType $(\mathrm{n}=14)$

\begin{tabular}{ccl}
\hline WaterBodyType_ID & ResourceType_ID & \multicolumn{1}{c}{ WaterBodyType } \\
\hline 0 & 0 & Unknown \\
1 & 4 & River/Stream \\
2 & 4 & Lake/Pond - surface water \\
3 & 4 & Reservoir \\
4 & 1 & Spring \\
5 & 1 & Aquifer - freshwater \\
6 & 5 & Estuary - brackish \\
7 & 6 & Estuary - saline \\
8 & 6 & Bay \\
9 & 6 & Ocean \\
10 & 1 & Pond - ground water \\
11 & 4 & Canal \\
12 & 4 & Surface water - undefined \\
13 & 2 & Aquifer - brackish \\
\hline
\end{tabular}


Appendix 2. NJWaTr Domain Table Listings by Subject Area-Continued

\section{Resource Domains-Continued}

tdsCriticalArea $(\mathrm{n}=4)$

\begin{tabular}{ccl}
\hline CriticalArea_ID & CriticalAreaCode & \multicolumn{1}{c}{ CriticalArea } \\
\hline 0 & 0 & Unknown \\
1 & 1 & Critical Area designated in the northeast Coastal Plain of New Jersey \\
2 & 2 & Critical Area designated in the Camden area of New Jersey \\
9 & 9 & Not Applicable \\
\hline
\end{tabular}

tdsCriticalZone $(\mathrm{n}=7)$

\begin{tabular}{ccl}
\hline CriticalZone_ID & CriticalZoneCode & \multicolumn{1}{c}{ CriticalZone } \\
\hline 0 & 0 & Unknown \\
1 & 1 & Well is in the depleted zone of a regulated aquifer \\
2 & 2 & Well is in the threatened margin of a regulated aquifer \\
3 & 3 & Well is within the composited area of a critical area but does not tap a \\
& 4 & regulated aquifer \\
4 & 5 & Well is not located in a critical area \\
5 & 9 & Well taps the depleted zone or threatened margin of a regulated \\
9 & & Not Applicable
\end{tabular}

tdxResourceDetailLabel (currently $n=5$ )

\begin{tabular}{cll}
\hline ResourceDetailLabel_ID & ResourceDetailLabel & ResourceDetailLabelMemo \\
\hline 1 & Tributary to & \\
2 & Surface area-acres & \\
3 & Dam name \\
4 & August median flow \\
5 & Fishery \\
\hline
\end{tabular}


Appendix 2. NJWaTr Domain Table Listings by Subject Area-Continued

\section{Miscellaneous Domains}

tdsTimeInterval $(\mathrm{n}=11)$

\begin{tabular}{cl}
\hline TimeInterval_ID & TimeInterval \\
\hline 1 & 5-year period \\
2 & Year \\
3 & Season \\
4 & Month \\
5 & Week \\
6 & Day \\
7 & Multi-day \\
8 & Indeterminate \\
9 & Hour \\
10 & Minute \\
11 & Second \\
\hline
\end{tabular}

tdsAddress Type $(\mathrm{n}=3)$

\begin{tabular}{cl}
\hline AddressType_ID & \multicolumn{1}{c}{ AddressType } \\
\hline 1 & Street \& Mailing \\
2 & Street \\
3 & Mailing \\
\hline
\end{tabular}

tdsOwnerType $(\mathrm{n}=7)$

\begin{tabular}{cll}
\hline OwnerType_ID & OwnerType & \multicolumn{1}{c}{ OwnerTypeMemo } \\
\hline 0 & Unknown & Unknown Owner Type \\
1 & None & No actual owner \\
2 & Private & Privately owned \\
3 & Municipal & Owned/operated by the Municipal government \\
4 & County & Owned/operated by the County government \\
5 & State & Owned/operated by the State government \\
6 & Federal & Owned/operated by the Federal government \\
\hline
\end{tabular}


Appendix 2. NJWaTr Domain Table Listings by Subject Area-Continued

Miscellaneous Domains-Continued

tdxDataSource (examples)

\begin{tabular}{ccll}
\hline DataSource_ID & Owner_ID & DataSource & \multicolumn{1}{c}{ DataSourceMemo } \\
\hline 0 & 0 & Unknown & Data Source is not known \\
1 & 2 & BWA & Bureau of Water Allocation \\
2 & 3 & SDWIS & Safe Drinking Water Information System \\
3 & 2 & NJGS revised BWA files & NJGS revised BWA files \\
4 & 2 & NJPDES & New Jersey Pollutant Discharge Elimination \\
& & System database \\
\hline
\end{tabular}

tdxPermitLabel (examples)

\begin{tabular}{|c|c|c|c|}
\hline PermitLabel_ID & DataSource_ID & PermitLabel & PermitLabelMemo \\
\hline 1 & 1 & BWA & \\
\hline 2 & 1 & Domestic Wells & \\
\hline 3 & 1 & DW Service Area & \\
\hline 4 & 4 & NJPDES & \\
\hline
\end{tabular}


Appendix 2. NJWaTr Domain Table Listings by Subject Area-Continued

Miscellaneous Domains-Continued

tdsPermitSeries $(\mathrm{n}=11)$

\begin{tabular}{|c|c|c|}
\hline PermitSeries_ID & PermitSeries & PermitSeriesDescription \\
\hline 0 & Unknown & Unknown Series \\
\hline 1 & 10000W registrations & Surface- and ground-water withdrawals at a rate less than or equal to 100,000 gallons per day \\
\hline 2 & 2000P and 2000E permits & Surface- and ground-water withdrawals for industrial, golf-course, and institutional use \\
\hline 3 & 4000PS permits & Surface-water withdrawals for industrial and public supply \\
\hline 4 & 5000 permits & Surface- and ground-water withdrawals for public supplies \\
\hline 5 & Agricultural certifications & Surface- and ground-water withdrawals for agricultural use \\
\hline 6 & Agricultural registrations & Surface- and ground-water withdrawals for agricultural use at a rate less than or equal to 100,000 gallons per day \\
\hline 7 & 1000D Dewatering permits & Temporary surface- or ground-water withdrawals required for a construction project \\
\hline 8 & Domestic Well permits & Aggregated and estimated ground-water withdrawals from domestic wells \\
\hline 9 & DW Service Area permits & Water distribution systems identified by one PWS ID \\
\hline 10 & NJPDES & New Jersey Permit Discharge Elimination System permit \\
\hline
\end{tabular}

tdxAliasLabel (example)

\begin{tabular}{ccccc}
\hline AliasLabel_ID & DataSource_ID & AliasSource & AliasLabel & AliasLabelMemo \\
\hline 1 & 6 & Dept. Civil Engineering & Rutgers Station ID & \\
\hline
\end{tabular}


Appendix 3. Comparison of the NJWaTr and NEWUDS Data Models 


\section{Appendix 3. Comparison of the NJWaTr and NEWUDS Data Models}

The differences between the NJWaTr data model and the original NEWUDS data model are described here. Some tables and fields were renamed, redefined, dropped from, or added to NEWUDS to create NJWaTr. Details of the differences are presented below by subject area along with a summary.

In addition to the items listed below, two general changes to the NEWUDS design have been implemented for NJWaTr; both are described in the "Operational Issues and Procedures" section of this report. (1) All non-associative tables are equipped in NJWaTr with a unique index involving one or more non-PK fields, whereas NEWUDS had only the mandatory indexes for PK and FK fields. (2) The maintenance queries are prefixed with the letters qrm in NJWaTr rather than the qry prefix used in NEWUDS. The names of some maintenance queries and views also were adjusted to reflect the renaming of tables or fields indicated below.

\section{Site Subject Area}

\section{$\underline{\text { Use Classification }}$}

- The USGS and New England use-classification terms in the nested domains tdsUSGSUseType and tdsNEUseType were dropped.

- Two new use-classification nested domains using classification terms provided by NJDEP were added--tdsUseGroup and tdsUseType.

- The $t d s S I C$ and $t d s N A I C S$ industrial-classification domains and their relationships to tblSite were dropped.

- The table tadUseConsumedFraction used optionally to store estimated monthly consumptive use percentages for different UseTypes was added. The table pairs a UseType with a Month (integer equivalent, where January $=1$ and December $=12$ ) and stores the fraction of water available that is assumed to be consumed during that month of the year for Sites of that type.

- The table tadSiteUseType used optionally to allow Sites with a primary UseType to be further identified as having secondary uses was added. The table pairs a Site with a UseType and the fractional estimate of that UseType in the overall classification of the Site.

\section{Site Details}

- The tasSiteTypeDetailLabel association table that pairs records in tdsSiteType and $t d x$ SiteDetailLabel was added to establish data-driven business rules that identify which labels are appropriate for which site types. The table can be used within a query or application interface to determine whether Sites of certain SiteTypes have the appropriate detail information stored in the database. 


\section{$\underline{\text { Site Permits }}$}

- NEWUDS used the Alias entity for all forms of alternative identifiers, including Permits, whereas the Permits are handled separately in NJWaTr to allow each to be associated with a Permit Series. Permits for Sites are handled through a new association table tasSitePermit that pairs Sites with Permit records in the new tblPermit table. (See below.)

\section{Conveyance Subject Area}

\section{Expected Transfer Amount}

- The tadConveyanceExpectedFraction table was added to handle the time-bounded estimate of the proportion in the From Site of total deliverable quantity expected to arrive at the Conveyance's To Site. Time-bounding allows the estimate to be amended whenever the From Site's Conveyances or expected deliveries change.

\section{Transfer/Volume Subject Area}

\section{Name Change}

- All terms using "Transaction" in the original NEWUDS model were changed to "Transfer" to better match the purpose of the NJDEP data model.

- All terms using "Rate" were changed to "Volume" because of the NJDEP decision to store quantity instead of rate (quantity/time) transfer values.

\section{Quantity data instead of Rate data}

- Transfer data are stored as quantities (for example, million gallons) rather than rates (such as million gallons per day).

- The Time component of the NEWUDS tdsRateUnit domain was dropped, leaving the Decimal and Quantity (formerly Volume) components as the only items that make up the clustered tdxVolumeUnit domain. 


\section{Location Subject Area}

\section{tblLocation}

- The NJEasting and NJNorthing fields were added to handle the NJ State Coordinate system.

\section{State Basin}

- The State Basin domain and association tables were dropped. These are not needed for New Jersey because the state uses standard hydrologic unit codes (HUCs) to designate watersheds.

\section{$\underline{\text { tdsState/County/MCD Domain values }}$}

- State, County, and MCD domains were changed from New England values to accommodate the NJ region (NJ, NY, PA, DE, MD) using data from the 2000 U.S. Census. Definitions were adjusted to reflect the NJWaTr content.

\section{Associations of Location with State, County, MCD, and HUC}

- In NEWUDS only the HUC domain had a tad association table connection to tblLocation, whereas the State, County, and MCD domains were direct FK joins.

- The tad tables pairing a Location to one or more State, County, and MCD (tadLocationState, tadLocationCounty, and tadLocationMCD, respectively) were added.

- The two non-PK fields in each of the tad tables are Fraction to indicate the percentage of the Location that is within each of the associated areas and IsPrimary to consistently identify and retrieve a single domain selection for the Location if more than one is associated.

\section{Drought Region}

- New Jersey has divided the State into drought regions. Each municipality belongs to only one drought region. The domain table tdsDroughtRegion was added to the model; it is a parent table to the $t d s M C D$ domain to provide drought region information for each municipality identified for a Location.

\section{$\underline{\text { tdsHUC }}$}

- The $t d s H U C$ domain in NEWUDS was only for 8-digit HUCs in New England. The NJWaTr HUC domain includes 8-, 11-, and 14-digit HUCs in a static array (no nesting of HUC levels using separate tables) covering the State of New Jersey. Fields were renamed to show HUC levels (HUCx, HUCxName).

- Definitions were adjusted to reflect the NJWaTr content.

- The domain contains 933 14-digit HUCs for NJ; 11-digit HUCs do not have standard names. 


\section{Water Region and Watershed Management Area}

- Two nested spatial areas for New Jersey are the Water Region and Watershed Management Area (see Hoffman and Lieberman, 2000). Because each domain has an official code and a name/description, these were added as nested tables (parent $t d s$ WaterRegion and child tdsWaterMgmntArea) and related to the $t d s H U C$ domain so that each 14-digit HUC is associated with a single Management Area and its parent Region.

\section{Resource Subject Area}

\section{$\underline{\text { Critical Area and Zone }}$}

- Two domains, tdsCriticalArea and tdsCriticalZone, were added to the tadSiteResource association table to identify diversion sources or withdrawals in either of two regulated aquifer areas defined within New Jersey. A "not applicable" selection can be assigned to Sites not associated with these spatial areas.

\section{$\underline{\text { Resource Owner }}$}

- An Owner was added to each Resource by adding Owner_ID as a mandatory, non-PK foreign key in the tblResource table. A "No Owner" selection can be assigned to a Resource that does not have a designated Owner.

\section{$\underline{\text { Resource Details }}$}

- Storage of Resource Details was set in tadResourceDetail to be time-bounded with the addition of the fields ResourceDetailEffectiveDate and ResourceDetailEndingDate.

- The PK of the tadResourceDetail table was adjusted to include the three fields, Resource_ID + ResourceDetailLabel_ID + ResourceDetailEffectiveDate, to allow multiple, time-bounded values for a single label.

\section{$\underline{\text { Resource Structure }}$}

- The association table tadResourceStructure was added to pair two records at a time from the tblResource table to show the component Resources for a composite Resource, along with the optional fractional amount of the parent represented by the child, and a designation of a primary child Resource for the composite parent.

\section{Water Body Type and Detail Label association}

- The table tasWaterBodyDetailLabel was added to pair records from WaterBodyType and ResourceDetailLabel in order to establish data-driven business rules that identify which labels are appropriate for different waterbody types. The table can be used within a query or application interface to determine whether Resources of certain WaterBodyTypes have the appropriate detail information stored in the database. 


\section{Permit Subject Area}

\section{$\underline{\text { Permits }}$}

- A new subject area focused on the tblPermit table was added to handle Permits assigned to Sites and Owners. Permit numbers were identified as critical to associations with other data sources and to retrievals. The Permit structure is similar to the Alias subject area, the latter having been retained in the NJWaTr model to handle all non-Permit alternative identification schemes for Sites, Conveyances, and Resources.

- The Permit structure includes the new domain tdsPermitSeries to classify each Permit according to a New Jersey scheme.

- Two new association tables join tblSite to tblPermit (tasSitePermit) and tblOwner to tblPermit (tasOwnerPermit) to associate individual Sites and Owners with individual Permits.

- Note that Permits are not assigned directly to Resources. Permits associated with Resources are gathered through the relationship between an individual Resource and an individual Site or Owner. Resource "allocations," if assigned directly to Resources, are handled through the tadResourceDetail table.

\section{Summary of differences between NJWaTr and NEWUDS}

\section{New Tables in NJWaTr $(n=21)$ listed alphabetically}

- tadConveyanceExpectedFraction

- tadLocationCounty

- tadLocationMCD

- tadLocationState

- tadResourceStructure

- tadSiteUseType

- tadUseConsumedFraction

- tasOwnerPermit

- tasSitePermit

- tasSiteTypeDetailLabel

- tasWaterBodyDetailLabel

- tblPermit

- tdsCriticalArea

- tdsCriticalZone

- tdsDroughtRegion

- tdsPermitSeries

- tdsUseGroup

- tdsUseType

- tdsWaterMgmntArea

- tdsWaterRegion

- $t d x$ PermitLabel 


\section{Dropped Tables from NEWUDS ( $\mathrm{n}=7$ ) listed alphabetically}

- tadLocationStateBasin

- tdsNAICS

- tdsNEUseType

- tdsRateUnitTime

- $t d s S I C$

- tdsStateBasin

- tdsUSGSUseType

\section{Changes to Tables that existed in the NEWUDS model}

- In the tblSite table, the SIC and NAICS domain foreign key fields were dropped.

- In the tblSite table, the foreign key NEUseType_ID was reset to the new domain UseType_ID .

- All Transaction terms were renamed Transfer.

- All Rate terms were renamed Volume, and the resulting VolumeUnitVolume term was renamed VolumeUnitQuantity.

- In the tdxVolumeUnit (formerly tdxRateUnit) table, the foreign key for the time component (RateUnitTime_ID) was dropped as a result of the switch from Rate to Volume storage.

- In the tblLocation table, NJNorthing and NJEasting state coordinate fields were added.

- The $t d s H U C, t d s$ State, $t d s C o u n t y$, and $t d s M C D$ tables were populated with New Jersey and surrounding states' items and codes.

- The $t d s H U C$ table was expanded for 8-, 11-, and 14-digit HUCS. The New Jersey WatershedCode was added, and a new WaterMgmntArea_ID foreign key from the new nested $t d s$ WaterMgmntArea and tdsWaterRegion domains was added.

- In the $t d s M C D$ table, a foreign key was added from the new tdsDroughtRegion domain.

- The tadSiteResource association table was amended to include foreign keys to the new domains tdsCriticalArea and tdsCriticalZone.

- The tblResource table was assigned an Owner with the addition of a foreign key Owner_ID field.

- The tadResourceDetail table was set in NJWaTr for time-bounding of detail values with the addition of the ResourceDetailEffectiveDate and ResourceDetailEndingDate fields. 

Tessler, Steven-DATA MODEL AND RELATIONAL DATABASE DESIGN FOR THE NEW JERSEY WATER-TRANSFER DATA SYSTEM (NJWaTr)U.S. Geological Survey Open-File Report 03-197 